\title{
APPLICATIONS OF THE THEORY OF BOOLEAN RINGS TO GENERAL TOPOLOGY*
}

BY

M. H. STONE

\section{INTRODUCTION}

In an earlier paper $\dagger$ we have developed an abstract theory of Boolean algebras and their representations by algebras of classes. We now relate this theory to the study of general topology.

The first part of our discussion is devoted to showing that the theory of Boolean rings is mathematically equivalent to the theory of locally-bicompact totally-disconnected topological spaces. In $\mathrm{R}$ we have already prepared the way for a topological treatment of the perfect representation of an arbitrary Boolean ring. Continuing in this way, we find that the perfect representation is converted by the introduction of a suitable topology into a space of the indicated type. We have no difficulty in inverting this result, proving that every locally-bicompact totally-disconnected topological space arises by the same procedure from a suitable Boolean ring. It is thus convenient to call the spaces corresponding in this manner to Boolean rings, Boolean spaces. The algebraic properties of Boolean rings can, of course, be correlated in detail with the topological properties of the corresponding Boolean spaces. A simple instance of the correlation is the theorem that the Boolean rings with unit are characterized as those for which the corresponding Boolean spaces are bicompact. A familiar example of a bicompact Boolean space is the Cantor discontinuum or ternary set, which we discuss at the close of Chapter I.

Having established this direct connection between Boolean rings and topology, we proceed in the second part of the discussion to considerations of a yet more general nature. We propose the problem of representing an arbitrary $T_{0}$-space by means of maps in bicompact Boolean spaces. Our solution of this problem embodies an explicit construction of such maps, which we shall now describe briefly. In a given $T_{0}$-space $\Re$, the open sets and the nowhere dense sets generate a Boolean ring, with $\Re$ as unit, which characterizes the topological structure of $\Re$. Those subrings which contain $\Re$ and which are so large that the interiors of their member sets constitute bases for $\Re$, also char-

* Presented to the Society (in part), February 25, 1933, and September 5, 1936; received by the editors June 1, 1936.

† Stone, these Transactions, vol. 40 (1936), pp. 37-111. In the sequel this paper will be designated by the letter $R$, citations being made in the form " $R$ Theorem 10 " or " $R$ Definition 6 ." 
acterize the structure of $\Re$. We are thus provided with an extensive family of Boolean rings which can be employed in the investigation of the given space $\Re$; we call them basic rings for $\Re$. In any basic ring for $\Re$, those sets with closure not containing a given point $\mathfrak{r}$ in $\Re$ constitute an ideal; and in the corresponding Boolean space, this ideal is represented by an open set with closed non-void complement $\mathfrak{X}(\mathfrak{r})$. The points of $\Re$ are thus represented by closed subsets of a related bicompact Boolean space. We find further that the topological structure of $\Re$ is characterized by the distribution of these representative closed sets. Thus we are able to reduce the study of general $T_{0^{-}}$ spaces to the examination of their maps in bicompact Boolean spaces. This reduction is not without advantage in the consideration of explicit topological problems, as we show in several illustrative applications.

The general mapping theory which we have outlined in the preceding paragraph is sufficiently complicated to suggest a search for simplifications. We turn naturally to the various strong separation properties such as regularity and normality. The investigation of the several possibilities which arise occupies the third chapter of our discussion. The general mapping theory, as previously developed, indicates the procedure for its own simplification and leads us at once to the consideration of a class of topological spaces to which little attention has been paid in the past. These spaces are characterized by the property that in them the regular open sets-that is, the interiors of closed sets-constitute bases. Since they are more general than the regular spaces, we call them semi-regular spaces. After discussing the semi-regular and regular spaces in detail, we consider the completely regular spaces. Here it is necessary for us to study the class of all bounded continuous real functions in a topological space. We obtain a reasonably complete algebraic insight into the structure of this class and its correlation.with the structure of the underlying topological space. We are thereby enabled to complete the study of the maps of completely regular spaces. The normal spaces are, from the point of view adopted here, so special that we do not devote any separate consideration to them.

Plainly, we are engaged here in building a general abstract theory and must accordingly be occupied to a considerable extent with the elaboration of technical devices. Such preoccupation appears the more unavoidable, because the known instances of our theory are so special and so isolated that they throw little light upon the domain which we have wished to investigate. Nevertheless, we have not neglected to test our theory by applying it to specific problems of general set-theoretical topology, some of which have remained unsolved for several years. We may note in particular the propositions established in Theorems 52 and 53. While these applications lie in the domain 
of set-theoretical topology, it is clear that the algebraic tendencies of our method relate it more nearly to recent developments in combinatorial topology. Indeed, it appears that by a process of gradual generalization combinatorial topologists have now arrived at a point of view very similar to that expounded here. Accordingly, we should expect that applications of the present theory could be made also to combinatorial problems. It seems clear, for instance, that the study of approximation by abstract complexes could conveniently be based upon the theory of Boolean maps, as developed here. We cannot, however, enter upon the discussion of such further applications at this time.

The present detailed exposition of our theory brings some corrections to previous summary announcements, as will be apparent from a reading of Chapter III, $\$ 1$ below. The simplification of the mapping theory which was originally stated to be possible in general is now recognized as characteristic for the semi-regular spaces.* The general theory is accordingly somewhat more complicated than we originally supposed; but its applications, so far as we have examined them, require no essential modification. There are some applications described in the announcement just cited which we do not consider in the present paper. They are chiefly the ones dealing with dimensiontheory. In Definitions 6 and 7 below we introduce the index of a map. The connections between the index and dimension-theory are pointed out in the indicated announcement, where references to the literature can be found. We hope to return to this subject at a later opportunity.

For general information concerning the elementary concepts of topology we refer the reader to the first two chapters of the recent book of Alexandroff and Hopf $\dagger$ and to a paper of Kuratowskił which deals with the algebraic properties of the closure operation. The latter paper will not be referred to explicitly below, since we shall give all the requisite algebraic reckonings in some detail. As we have already indicated, we shall confine our attention to the $T_{0}$-spaces. Our reason for doing so is that, among all the topological spaces which can be defined in terms of closure or of neighborhood systems, the $T_{0}$-spaces appear to be essentially the most general spaces which are of real interest. The $T$-spaces, as we have pointed out elsewhere, $\S$ are more general than the $T_{0}$-spaces but only from a logical, rather than a topological, point of view: if one identifies the topologically indistinguishable points in a $T$-space, the result is a $T_{0}$-space.

${ }^{*}$ Note the oversimplifications in Theorems $\mathrm{V}_{1}, \mathrm{~V}_{2}$, and $\mathrm{V}_{3}$ in the Proceedings of the National Academy of Sciences, vol. 20 (1934), pp. 197-202.

$\dagger$ Alexandroff and Hopf, Topologie I, Leipzig, 1935, cited hereafter by the letters AH.

$\ddagger$ Kuratowski, Fundamenta Mathematicae, vol. 3 (1922), pp. !82-199.

$\S$ Stone, Matematicheskii Sbornik, vol. 1 (old series, vol. 43), . No. 5 (1936-37). 
The notations of the present paper are largely determined by the usages already established in $\mathrm{R}$. Thus, for instance, in considering Boolean rings as abstract entities, we continue to use the symbols $\cdot, v,+,<,{ }^{\prime}$ corresponding to the symbols $\cdot, \cup, \Delta, \subset,{ }^{\prime}$, of the theory of classes. In the algebra of the closure operation, we write $A^{-}$in place of $\bar{A}$ for convenience in putting down the more complicated expressions such as $A^{-1-1}$.

The contents of the present paper may be summarized systematically under the following headings: Chapter I, Boolean spaces: $\$ 1$, Introduction of topological concepts; §2, Relations between algebra and topology; §3, Universal Boolean rings and spaces. Chapter II, Maps in Boolean spaces: $\S 1$, The general theory of maps; $\$ 2$, Construction of Boolean maps; $\$ 3$, Relation between algebraic and other maps; $\$ 4$, Applications to the theory of extensions; $\$ 5$, Totally-disconnected and discrete spaces. Chapter III, Stronger separation conditions: $\S 1$, Semi-regular spaces; $\$ 2$, Regular spaces; $\$ 3$, Completely regular spaces.

\section{Chapter I. Boolean SPaCes}

1. Introduction of topological concepts. We commence with the introduction of a topology in the abstract class in which the perfect representation of a Boolean ring has been constructed as described in $\mathrm{R}$ Chapter IV. It is natural to impose upon $\mathbb{E}$, the class of all prime ideals in a Boolean ring $A$, a neighborhood topology based upon the special subclasses $\mathbb{E}(\mathfrak{a})$, where $\mathfrak{a}$ is an ideal in $A$, or upon the still more restricted subclasses $\mathbb{E}(a)=\mathbb{E}(\mathfrak{a}(a))$, where $a$ is an arbitrary element in $A$. The consequences of this procedure are stated in the following theorem.

TheOREM 1. Let $A$ be a Boolean ring, a an arbitrary ideal in $A$, \& the class of all prime ideals in $A, \mathbb{E}(\mathfrak{a})$ the class of all prime ideals which are not divisors of $\mathfrak{a}$, and $\mathfrak{E}(a)$ the class $\mathfrak{E}(\mathfrak{a}(a))$ corresponding to the principal ideal $\mathfrak{a}=\mathfrak{a}(\boldsymbol{a})$. The topologies imposed upon \& through the introduction of the neighborhood systems (1) and (2), where

(1) each $\mathfrak{E}(\mathfrak{a})$ is assigned as a neighborhood of every element which it contains;

(2) each $\mathbb{E}(a)$ is assigned as a neighborhood of every element which it contains;

are equivalent. Under them, \& is a topological space with the properties:

(1) \& is a totally-disconnected, ${ }^{*}$ locally-bicompact $H$-space;

* We use the term "totally-disconnected" to mean that, whenever $\mathfrak{p}$ and $q$ are distinct points of a topological space, there exist disjoint closed sets which contain $\mathfrak{p}$ and $q$ respectively and which have the entire space as their union. It is clear that a totally-disconnected topological space is necessarily an $H$-space. 
(2) the classes $\mathbb{E}(\mathfrak{a})$ are characterized as the open sets in $\mathbb{E}$;

(3) the classes \& (a) are characterized as the bicompact open sets in $\mathbb{E}$. The character of $\&$ is equal to the cardinal number of $A$ whenever $A$ is infinite. The space $\&$ is bicompact if and only if $A$ has a unit $e$.

The algebraic properties of the sets $\&(\mathfrak{a})$ and $\mathbb{E}(a)$ are sufficient to justify the introduction of the systems (1) and (2) as neighborhood-systems. In fact, the sets $\mathbb{E}(\mathfrak{a})$ have the following properties: an arbitrary element $\mathfrak{p}$ in $\mathbb{E}$, being a prime ideal in $A$, has as its neighborhoods the classes $\mathbb{E}(\mathfrak{a})$ where $\mathfrak{p} \not \mathfrak{a}$, and is contained in each of its neighborhoods; if $\mathbb{E}(\mathfrak{a})$ and $\mathbb{E}(\mathfrak{b})$ are neighborhoods of $\mathfrak{p}$, then their intersection $\mathbb{E}(\mathfrak{a} \mathfrak{b})=\mathfrak{E}(\mathfrak{a}) \mathfrak{E}(\mathfrak{b})$ is also a neighborhood of $\mathfrak{p}$; and if $\mathbb{E}(\mathfrak{a})$ contains $\mathfrak{p}$, then $\mathbb{E}(\mathfrak{a})$ is a neighborhood of $\mathfrak{p}$. Thus the system (1) has the properties demanded of a neighborhood-system in a topological space; ${ }^{*}$ under it $\&$ becomes a topological space. Under the system (1), the sets $\mathbb{E}(a)$ are special open sets. Since the classes $\mathbb{E}(\mathfrak{a})$ are characterized algebraically as unions of classes $\mathbb{E}(a)$ by virtue of the fact, established in $\mathrm{R}$ Theorem 67 (2), that the relations $\mathfrak{a}=\mathrm{S}_{a \in \mathfrak{a}} \mathfrak{a}(a)$ and $\mathbb{E}(\mathfrak{a})=\sum_{a \in a} \mathfrak{E}(a)$ are equivalent, we see that the classes $\&(a)$ constitute a basis in the topological space $\mathbb{E}$. In consequence, the assignment of these sets as neighborhoods in accordance with (2) provides a neighborhood-system equivalent to the system (1). Moreover, it is evident that the classes $\mathbb{E}(\mathfrak{a})$ are characterized as the open subsets of $\&$ under these equivalent neighborhood-systems. The system (1) is identified in this way as the absolute neighborhood-system. $\dagger$

The nature of the topological space $\&$ is now easily determined. To show that it is totally-disconnected, we start with distinct points $p$ and $q$ in $\mathbb{E}$ and construct ideals $\mathfrak{a}$ and $\mathfrak{b}$ so that $\mathfrak{p} \in \mathbb{E}(\mathfrak{a}), \mathfrak{q} \in \mathbb{E}(\mathfrak{b}), \mathbb{E}(\mathfrak{a}) \mathbb{E}(\mathfrak{b})=0, \mathbb{E}(\mathfrak{a}) \cup \mathfrak{E}(\mathfrak{b})=\mathbb{E}$. Since $\mathfrak{p}$ and $q$ are distinct prime ideals in $A$, there exists an element $a$ which belongs to $\mathfrak{q}$ but not to $\mathfrak{p}$. If we now put $\mathfrak{a}=\mathfrak{a}(a), \mathfrak{b}=\mathfrak{a}^{\prime}(a)$, we see that $\mathfrak{p} \boldsymbol{p} \mathfrak{a}$, $\mathfrak{q} \not \mathfrak{b}, \mathfrak{a} \mathfrak{b}=\mathfrak{b}, \mathfrak{a} \vee \mathfrak{b}=\mathfrak{e}$; and we can rewrite these relations in the desired form. Now the sets $\mathbb{E}(\mathfrak{a}), \mathfrak{E}(\mathfrak{b})$ are open in $\mathbb{E}$; since they are mutually complementary, they are also closed in $\mathbb{E}$. Thus $\mathbb{E}$ is seen to be totally-disconnected; and, in particular, to be an $H$-space. It remains for us to prove that $\&$ is locally-bicompact. We shall do so by showing that every neighborhood $\mathbb{E}(a)$ in the system (2) is a bicompact subspace of $(E$. The open sets in the subspace $\mathbb{E}(a)$ are precisely the sets $\mathbb{E}(\mathfrak{a}) \&(a)$. The Heine-Borel-Lebesgue covering property, which is to be proved for $\mathbb{E}(a)$, assumes the following form: if $\mathfrak{A}$ is any class of ideals $a$ such that $\sum_{\text {aet }} \mathbb{E}(\mathfrak{a}) \mathbb{E}(a)=\mathbb{E}(a)$, then $\mathfrak{A}$ contains ideals $\mathfrak{a}_{1}, \cdots, \mathfrak{a}_{n}$ such that $\sum_{\nu=1}^{\nu-n} \mathfrak{E}\left(\mathfrak{a}_{v}\right) \mathbb{E}(a)=\mathbb{E}(a)$. The desired property is equiva-

\footnotetext{
* AH, p. 43, Satz IX.

$\dagger$ AH, p. 42.
} 
lent to the following algebraic property: if $a \epsilon \mathrm{S}_{a \in \ell} \mathfrak{a}$, then $a \epsilon \mathrm{S}_{\nu=1}^{\nu-n} \mathfrak{a}_{\nu}$ for appropriate ideals. $\mathfrak{a}_{1}, \cdots, \mathfrak{a}_{n}$ in $\mathfrak{A}$. The latter property has been established in $\mathrm{R}$ Theorem 17. Conversely, we can show that an open subset $\mathbb{E}(\mathfrak{a})$ of $\mathbb{E}$ which, when considered as a subspace, is bicompact, is necessarily a set $\mathbb{E}(a)$. The sets $\mathbb{E}(a)$ contained in $\mathbb{E}(\mathfrak{a})$ are open in this subspace; and they are precisely those sets corresponding to the elements $a$ of the ideal $a$. The relation $\mathbb{E}(\mathfrak{a})=\sum_{a \in \mathfrak{a}} \mathbb{E}(a)$ yields elements $a_{1}, \cdots, a_{n}$ in $\mathfrak{a}$ such that $\mathbb{E}(\mathfrak{a})=\sum_{\nu-1}^{\nu-n} \mathbb{E}\left(a_{\nu}\right)$ in accordance with the postulated Heine-Borel-Lebesgue covering property. The latter relation is equivalent to $\mathbb{E}(\mathfrak{a})=\mathbb{E}(a)$ where $a=a_{1} \vee \cdots \vee a_{n}$. This completes the topological characterization of the sets $\mathbb{E}(a)$. It may be remarked that every $\mathbb{E}(a)$, being bicompact, is necessarily a closed subset of $\mathbb{E}$.

The character of the space $\&$ can now be determined without difficulty. In case $A$ is a finite ring, the space $E$ consists of $n$ points where $2^{n}$ is the number of elements in $A$ and $n \geqq 0$; every subset of $\&$ is an open set and its points are all isolated. In case $A$ has an infinite cardinal number $\mathrm{c}$, the neighborhood-system (2) also has cardinal number c. Consequently the character of $\&$ (that is, the least cardinal number belonging to a basis in $\&$ ) cannot exceed $c$. On the other hand, if the open sets $\mathcal{G}_{\alpha}$, where the index $\alpha$ runs over a fixed abstract class $\mathrm{A}$, constitute a basis in $\mathbb{E}$, every set $\mathbb{E}(a)$ is the union of appropriate sets $\mathfrak{B}_{\alpha}, \alpha \in \mathrm{A}$. The bicompactness of $\mathbb{E}(a)$ thus yields indices $\alpha_{1}, \cdots, \alpha_{n}$ such that $\mathbb{E}(a)=\sum_{\nu=1}^{\nu-n} \mathfrak{S}_{\alpha_{\nu}}$. Since every $\mathbb{E}(a)$ is thus the union of a finite number of sets from the given basis, the cardinal number of the class of all sets $\&(a)$ does not exceed that of the basis in question. We thus see that the character of $\&$ is equal to $c$.

If $A$ has a unit $e$, then $\&=\&(e)$ is bicompact in accordance with the preceding results. On the other hand, if $\mathbb{E}=\mathbb{E}(\mathrm{e})$ is bicompact, then $\mathrm{e}$ is a principal ideal, $\mathfrak{e}=\mathfrak{a}(a)$; and its generating element $e=a$ is a unit in $A$.

We proceed immediately to establish the converse of Theorem 1.

TheOReм 2. If $\subseteq$ is a totally-disconnected locally-bicompact $H$-space, then the bicompact open subsets of $\subseteq$ constitute a Boolean ring $A$; and, if the class $\mathbb{E}$ of prime ideals in $A$ is topologized as in Theorem 1, then $\mathbb{E}$ and $\subseteq$ are topologically equivalent.

We shall first show that the subsets of $\subseteq$ which have been designated as members of $A$ constitute a basis in $\subseteq$. If $\&$ is any point in $\subseteq$ and $(\mathcal{B}$ any open set containing 8, we have to establish the existence of a set $\mathfrak{S}$ in $A$ such that

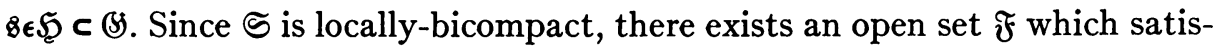
fies the relation $\mathbb{z} \in \mathfrak{F} \subset \mathbb{S}$ and which has closure $\mathfrak{F}^{-}$with the property of bicompactness. If $\mathfrak{F}=\mathfrak{F}^{-}$, we may put $\mathfrak{S}=\mathfrak{F}$. Otherwise we take advantage of the total-disconnectedness of $\mathfrak{S}$ : if $t$ is any point in $\mathfrak{F}^{-} \mathfrak{F}^{\prime} \neq 0$, then $8 \neq t$; 
and there exists a set $\mathbb{S}(t)$ which contains $t$ but not 8 and which, together with its complement, is both open and closed in $\mathfrak{S}$. Since $\mathfrak{F}^{-}$is bicompact, the relation $\mathfrak{F}^{-}=\mathfrak{F}^{-} \mathfrak{F} \cup \sum_{\text {te } \mathfrak{F}^{-} \mathfrak{F}^{\prime}} \mathfrak{F}^{-} \mathfrak{S}(\mathrm{t})$, in which $\mathfrak{F}$ and $\mathfrak{S}(\mathrm{t})$ are open sets, implies the existence of points $t_{1}, \cdots, t_{n}$ in $\mathfrak{F}^{-} \mathfrak{F}^{\prime}$ such that $\mathfrak{F}^{-}=\mathfrak{F}^{-} \mathfrak{F} \cup \sum_{\nu=1}^{\nu-n} \mathfrak{F}^{-} \mathfrak{S}\left(\mathfrak{t}_{v}\right)$. We now put $\mathfrak{S}=\mathfrak{F}^{\prime} \mathfrak{S}^{\prime}\left(t_{1}\right) \cdots \mathfrak{S}^{\prime}\left(\mathrm{t}_{n}\right)=\mathfrak{F}^{-} \mathfrak{S}^{\prime}\left(\mathrm{t}_{1}\right) \cdots \mathfrak{S}^{\prime}\left(\mathrm{t}_{n}\right)$. It is evident that $\mathfrak{S}$ is both open and closed in $\mathfrak{S}$, that it contains $\mathbb{B}$, and that it is contained in both $\mathfrak{F}$ and $\mathfrak{F}$. Since $\mathfrak{S}$ is closed in $\subseteq$ and coincides with $\mathfrak{S} \mathfrak{F}^{-}$, it is also closed in $\mathfrak{F}^{-}$. Thus $\mathfrak{S}$ is bicompact when considered as a subspace of $\mathfrak{F}^{-}$; since the topology of $\mathfrak{S}$ is the same whether $\mathfrak{S}$ be regarded as a subspace of $\mathfrak{F}^{-}$or of $\mathfrak{S}, \mathfrak{W}$ is bicompact also with respect to $\mathfrak{S}$. We thus complete the proof that $\subseteq$ has the desired basis.

We shall next show that the subsets of $\subseteq$ which are designated as members of $A$ constitute a perfect algebra of classes in $\mathfrak{S}$, in accordance with $\mathrm{R}$ Definition 12. It is immediately evident that $A$ contains $\mathfrak{A} \mathfrak{B}$ and $\mathfrak{A} \Delta \mathfrak{B}$ together with $\mathfrak{A}$ and $\mathfrak{B}$ : for $\mathfrak{A} \mathfrak{B}$ is open since it is the intersection of open sets and bicompact since it is closed in the bicompact sets $\mathfrak{A}$ and $\mathfrak{B}$ respectively; the set $\mathfrak{A} \cup \mathfrak{B}$ is bicompact; and the set $\mathfrak{A} \Delta \mathfrak{B}=\mathfrak{A} \mathfrak{B}^{\prime} \cup \mathfrak{A}^{\prime} \mathfrak{B} \subset \mathfrak{A} \cup \mathfrak{B}$ is both open and closed in $\subseteq$ and is bicompact since it is closed in the bicompact space $\mathfrak{A} \cup \mathfrak{B}$. Thus $A$ is a Boolean ring with classes as elements. Since $A$ is a basis in the $H$-space $\mathfrak{S}$, it is a reduced algebra of classes in accordance with $\mathrm{R}$ Definition 10: each point of $\subseteq$ is contained in some member of $A$ and is the sole point common to all such members of $A$. In order to show that $A$ is perfect, we prove that $\sum_{\mathfrak{I e}_{e} \mathfrak{A}} \mathfrak{A}=\sum_{\mathfrak{B} \mathfrak{e}} \mathfrak{B}$ implies $\mathfrak{a}=\mathfrak{b}$ whenever $\mathfrak{a}$ and $\mathfrak{b}$ are ideals in $A$. It is evidently sufficient to prove that $\sum_{\mathscr{q} e a} \mathfrak{A} \subset \sum_{\mathbb{B}_{\epsilon} b} \mathfrak{B}$ implies $\mathfrak{a} \subset \mathfrak{b}$. If $\mathfrak{A}$ is an arbitrary element of $\mathfrak{a}$, we have $\mathfrak{A} \subset \sum_{\mathfrak{B} \in \mathfrak{b}} \mathfrak{B}$ by hypothesis. Hence we can write $\mathfrak{A}=\sum_{\mathfrak{B} \in \mathfrak{E}} \mathfrak{Y} \mathfrak{B}$ and even, by virtue of the bicompactness of $\mathfrak{A}$, the stronger relation $\mathfrak{A}=\sum_{\nu=1}^{\nu-n} \mathfrak{A} \mathfrak{B}_{\nu}$ where $\mathfrak{B}_{1}, \cdots, \mathfrak{B}_{n}$ are in $\mathfrak{b}$. Since the latter relation implies $\mathfrak{A} \in \mathfrak{b}$, we conclude that $\mathfrak{a} \subset \mathfrak{b}$. Thus $A$ is perfect in accordance with R Definition 12.

Now by $\mathrm{R}$ Theorem 69 , the algebra $A$ is equivalent to its perfect representation $B(A)$ : in other words, there exists a biunivocal correspondence between $\subseteq$ and $\mathbb{E}=\mathbb{E}(A)$ which carries the classes $\mathfrak{A}$ belonging to $A$ into their respective representative classes $\mathfrak{E}(\mathfrak{A})$ in a biunivocal manner. Since $A$ is a basis in $\subseteq$ and the classes $\mathbb{E}(\mathfrak{H})$ constitute a basis in $\mathbb{E}$ by Theorem 1 , we conclude that $\subseteq$ and $\mathbb{E}$ are topologically equivalent.

The correspondence between Boolean rings and topological spaces established in Theorems 1 and 2 justifies the introduction of the following definitions and notations.

Definition 1. A totally-disconnected locally-bicompact $H$-space is called a Boolean space. 
Definition 2. A Boolean space $B$ is called a representative of a Boolean ring $A$, symbolically, $\mathfrak{B}=\mathfrak{B}(A)$, if $\mathfrak{B}$ is topologically equivalent to the particular Boolean space $\mathbb{E}=\mathbb{E}(A)$ described in Theorem 1 .

Before developing further details of the relations connecting Boolean rings and their representative Boolean spaces, we note a few useful topological facts.

THEOREM 3. The closed subsets and the open subsets of a Boolean space are Boolean spaces. A continuous image of a bicompact Boolean space is necessarily a bicompact topological space; it is therefore a Boolean space if and only if it is totally-disconnected.

Let $\mathfrak{F}$ be a closed subset of a Boolean space $\mathfrak{B}$. If $\mathfrak{x}_{1}$ and $\mathfrak{x}_{2}$ are distinct points in $\mathfrak{F}$, then there exist closed sets $\mathfrak{F}_{1}$ and $\mathfrak{F}_{2}$ such that $\mathfrak{x}_{1} \in \mathfrak{F}_{1}, \mathfrak{x}_{2} \in \mathfrak{F}_{2}$, $\mathfrak{F}_{1} \mathfrak{F}_{2}=0, \mathfrak{F}_{1} \cup \mathfrak{F}_{2}=\mathfrak{B}$. The sets $\mathfrak{S}_{1}=\mathfrak{F}_{\mathfrak{F}_{1}}, \mathfrak{S}_{2}=\mathfrak{F}_{2}$ are closed in $\mathfrak{F}$ and have the properties $\mathfrak{x}_{1} \in \mathfrak{S}_{1}, \mathfrak{x}_{2} \in \mathfrak{S}_{2}, \mathfrak{S}_{1} \mathfrak{S}_{2}=0, \mathfrak{S}_{1} \cup \mathfrak{S}_{2}=\mathfrak{F}$. Hence the space $\mathfrak{F}$ is totallydisconnected; in particular $\mathfrak{F}$ is an $H$-space. If $\mathfrak{S}$ is any open subset of $\mathfrak{F}$ and $\mathfrak{x}$ is any point in $\mathfrak{S}$, then $\mathfrak{S}=\mathscr{B} \mathfrak{F}$ where $\mathfrak{B}$ is open in $\mathfrak{B}$. Since $\mathbb{S}$ contains $\mathfrak{x}$, there exists an open set $\mathscr{S}_{1}$ which has the property $\mathfrak{x} \in \mathscr{G}_{1} \subset \mathbb{G}$ and possesses a bicompact closure $\mathfrak{G}_{1}^{-}$in $\mathfrak{B}$. The set $\mathfrak{S}_{1}=\mathfrak{G}_{1} \mathfrak{F}$ is open in $\mathfrak{F}$ and has the property $\mathfrak{x} \in \mathfrak{S}_{1} \subset \mathfrak{S}$. Furthermore its closure in $\mathfrak{F}$ is the closed set $\left(\mathfrak{G}_{1} \mathfrak{F}\right)-\mathfrak{F} \subset \mathfrak{G}_{1}^{-} \mathfrak{F} \subset \mathfrak{S}_{1}^{-}$; being closed also in $\mathfrak{S}_{1}^{-}$, it is bicompact. Thus $\mathfrak{F}$ has all the properties of a Boolean space.

Let $(S)$ be an open subset of a Boolean space $\mathfrak{B}$. Then $\mathbb{B}$ is a totallydisconnected $H$-space by the same reasoning as that applied to $\mathfrak{F}$ in the preceding paragraph. The proof that $\&$ is locally-bicompact proceeds as follows: the open sets in the subspace $B$ are precisely the open sets of $\mathscr{B}$ which are contained in $(B)$; if $\mathfrak{x}$ is any point of $B$ and $\mathscr{S}_{1}$ an open set such that $\mathfrak{x} \in \mathbb{S}_{1} \subset \mathbb{S}$, then there exists an open set $\mathfrak{S}$ which has the property $\mathfrak{x} \in \mathfrak{S} \subset \mathbb{S}_{1} \subset \mathbb{B}$ and possesses a bicompact closure $\mathfrak{S}^{-}=\mathfrak{F}$; and the closure of $\mathfrak{S}$ in the space (B) is given by $\mathfrak{S}-(\mathfrak{S})=\mathfrak{S}(\mathfrak{S}=\mathfrak{S}$.

It is well known that every continuous image of a bicompact $H$-space is a bicompact topological space. ${ }^{*}$ The final statement of the theorem is thus obvious.

A proof of Theorem 3 can also be obtained in an indirect way from the correspondence between Boolean rings and Boolean spaces, as will be seen in the following section; but it is preferable to have the direct proof before us in stating our results.

2. Relations between algebra and topology. We shall now develop in

* AH, p. 95, Satz 1. 
much greater detail the consequences of the relations between algebra and topology established in $\$ 1$. We have the following general theorem.

THEOREM 4. The algebraic theory of Boolean rings is mathematically equivalent to the topological theory of Boolean spaces by virtue of the following relations:

(1) every Boolean ring has a representative Boolean space; every Boolean space is the representative of some Boolean ring; and two Boolean rings are isomorphic if and only if their representatives are topologically equivalent;

(2) the group of automorphisms of a Boolean ring is isomorphic to the topological group of an arbitrary representative of the ring;

(3) the representatives of Boolean rings which are isomorphic to the various ideals in a Boolean ring $A$ are characterized topologically as the open subsets of an arbitrary representative of $A$; in particular, $\mathfrak{E}(\mathfrak{a})$ is a representative of the ideal a in $A$;

(4) the representatives of the homomorphs of a Boolean ring $A$ are characterized topologically as the closed subsets of an arbitrary representative of $A$; in particular, $\mathfrak{E}^{\prime}(\mathfrak{a})$ is a representative of the quotient ring $A / \mathfrak{a}$;

(5) the representatives of Boolean rings with unit are characterized topologically by the property of bicompactness.

This theorem merely collects in a new form results already proved in $\mathrm{R}$ and in Theorems 1 and 2 above. To establish (1) we merely compare $R$ Theorem 69, Theorem 1, and Theorem 2 . We then see that (2) follows directly from (1). We have already established (5) as part of Theorem 1. Using (1) in conjunction with Theorem 1 (2), we see that (3) and (4) are established as soon as we can prove the special assertions to the effect that $\mathbb{E}(\mathfrak{a})$ and $\mathbb{F}^{\prime}(\mathfrak{a})$ are representatives of $\mathfrak{a}$ and $A / \mathfrak{a}$ respectively. To show that $\mathbb{E}(\mathfrak{a})$ is a representative of $\mathfrak{a}$, considered as a Boolean ring, we need only show that the sets $\mathbb{E}(a), a \in \mathfrak{a}$, constitute a perfect algebra of classes in $\mathbb{E}(\mathfrak{a})$ isomorphic to the ring $\mathfrak{a}$. Now the ideals in the ring $\mathfrak{a}$ are precisely the ideals $\mathfrak{b}$ in $A$ such that $\mathfrak{b} \subset \mathfrak{a}$; and the relation $\mathfrak{b} \subset \mathfrak{a}$ is equivalent by $\mathrm{R}$ Theorem 67 to the relation $\mathbb{E}(\mathfrak{b}) \subset \mathbb{E}(\mathfrak{a})$. That theorem shows further that the sets $\mathbb{E}(\mathfrak{b})$ contained in $\mathbb{E}(\mathfrak{a})$ constitute an algebra of classes isomorphic to the algebra of all ideals in $\mathfrak{a}$ in the precise sense stated there. On specializing to the sets $\mathbb{E}(a)$ corresponding to the principal ideals $\mathfrak{b}=\mathfrak{a}(a), a \in \mathfrak{a}$, in the ring $\mathfrak{a}$, we see that these sets constitute an algebra of classes which is reduced and perfect in accordance with R Definitions 10 and 12 . It should be noted that the sets $\mathbb{E}(\mathfrak{b})$ contained in $\mathbb{E}(\mathfrak{a})$ are precisely the open sets in the subspace $\mathbb{E}(\mathfrak{a})$ of $\mathbb{E}$ : for every such open set is expressible, in harmony with Theorem 1 and $\mathrm{R}$ Theorem 67 , in the form $\mathfrak{E}(\mathfrak{b})=\mathfrak{E}(\mathfrak{c}) \mathfrak{E}(\mathfrak{a})$, where $\mathfrak{c}$ is an arbitrary ideal in $A$ 
and $\mathfrak{b}=\mathfrak{c} \mathfrak{a}$ is an ideal contained in $\mathfrak{a}$. The proof that $\mathfrak{E}^{\prime}(\mathfrak{a})$ is a representative of $A / \mathfrak{a}$ is similar. We know from $\mathrm{R}$ Theorem 68 that the sets $\mathcal{E}(a) \mathcal{E}^{\prime}(\mathfrak{a})$ constitute a reduced perfect algebra of classes in $\mathbb{E}^{\prime}(\mathfrak{a})$ isomorphic to $A / \mathfrak{a}$; and that the sets $\mathbb{E}(\mathfrak{b}) \mathbb{F}^{\prime}(\mathfrak{a})$ constitute an algebra of classes isomorphic to the algebra of the ideals in $A / \mathfrak{a}$. The latter sets are precisely the open sets in the space $\mathbb{E}^{\prime}(\mathfrak{a})$. The desired result is thus established. We note that the relations between the spaces $\mathfrak{F}(\mathfrak{a}), \mathfrak{F}^{\prime}(\mathfrak{a})$ and the Boolean rings $\mathfrak{a}, A / \mathfrak{a}$ imply that these spaces are Boolean spaces. Combining this fact with Theorem 1 (2), we obtain an indirect proof of the first part of Theorem 3.

It is a matter of some interest to characterize topologically the various classes of ideals introduced in $\mathrm{R}$ Definition 8 . We find the following result, which is most conveniently stated in terms of the perfect representation $\mathbb{E}(A)$.

TheOREM 5. If $\mathfrak{a}$ is an ideal in a Boolean ring $A$, then $\mathfrak{E}\left(\mathfrak{a}^{\prime}\right)$ coincides with the exterior of $\mathfrak{E}(\mathfrak{a})$. Hence the sets $\mathbb{E}(\mathfrak{a})$ corresponding to ideals in the respective classes $\mathfrak{\Im}, \mathfrak{R}, \mathfrak{S}, \mathfrak{B}^{*}, \mathfrak{B}$ of $\mathrm{R}$ Definition 8 are characterized topologically as follows:

(1) the sets $\mathbb{E}(\mathfrak{a})$ corresponding to arbitrary ideals are the open sets in $\mathbb{E}$;

(2) the sets $\mathbb{E}(\mathfrak{a})$ corresponding to normal ideals are the regular open sets in 8 ;

(3) the sets $\mathbb{E}(\mathfrak{a})$ corresponding to simple ideals are the open-and-closed sets in $\&$, or, equivalently, the sets in \& with void boundaries;

(4) the sets $\mathbb{E}(\mathfrak{a})$ corresponding to semiprincipal ideals are the open sets in $\&$ which are bicompact or are complements of bicompact open sets;

(5) the sets $\mathbb{E}(\mathfrak{a})$ corresponding to principal ideals are the bicompact open sets in $\mathbb{E}$.

We take (1) directly from Theorem 1 (2). It is then obvious from $R$ Definition 7 and $\mathrm{R}$ Theorem 19 that $\mathbb{E}\left(\mathfrak{a}^{\prime}\right)$ is the maximal open set disjoint from $\mathbb{E}(\mathfrak{a})$; in other words, $\mathfrak{E}\left(\mathfrak{a}^{\prime}\right)$ coincides with $\mathbb{E}^{-\prime}(\mathfrak{a})$, the exterior of $\mathbb{E}(\mathfrak{a})$. Since $\mathfrak{a}^{\prime \prime}=\mathfrak{a}$ if and only if $\mathfrak{E}\left(\mathfrak{a}^{\prime \prime}\right)=\mathfrak{E}(\mathfrak{a})$, we see that the normal ideals are characterized by the relation $\mathbb{E}(\mathfrak{a})=\mathbb{E}^{--^{\prime}}(\mathfrak{a})$, which identifies their representative open sets as the regular open sets. The simple ideals $a$ are those for which $\mathfrak{a} \vee \mathfrak{a}^{\prime}=\mathfrak{e}$, or, equivalently, $\mathbb{E}(\mathfrak{a}) \cup \mathbb{E}\left(\mathfrak{a}^{\prime}\right)=\mathbb{E}$. Hence they are characterized by the property $\mathbb{E}^{\prime}(\mathfrak{a})=\mathbb{E}\left(\mathfrak{a}^{\prime}\right)$ or $\mathfrak{E}(\mathfrak{a})=\mathfrak{E}-(\mathfrak{a})$. Their representative sets are thus characterized by the property of being closed as well as open. Now in any topological space, the relations $\mathfrak{S}=\mathfrak{S}^{-}, \mathfrak{S}^{\prime}=\mathfrak{S}^{\prime}$, which express the fact that $\mathfrak{S}$ is both closed and open, are together equivalent to the relation $\mathfrak{S}^{-} \mathfrak{S E}^{\prime}-=0$ which expresses the fact that the boundary of $\mathfrak{S}$ is void: for, when the first relations hold, we have $\mathfrak{S}^{-} \mathfrak{S}^{\prime}-=\mathfrak{S G S}^{\prime}=0$; and, when the second relation holds, we have $\mathfrak{S} \subset \mathfrak{S}^{-}, \mathfrak{S E}^{\prime} \subset \mathfrak{S g}^{\prime-}, \mathfrak{S}^{-} \mathfrak{S E}^{\prime}=0$, and hence $\mathfrak{S}=\mathfrak{S E}^{-}$, $\mathfrak{S E}^{\prime}=\mathfrak{S}^{\prime}$. Thus the simple ideals $\mathfrak{a}$ are characterized by the fact that the 
corresponding sets $\&(\mathfrak{a})$ have void boundaries, as stated in (3). We pass next to the consideration of (5). Here we have merely repeated the characterization already given in Theorem 1 . Then by combining (3) and (5) we obtain (4), since an ideal $\mathfrak{a}$ is semiprincipal if and only if both ideals $\mathfrak{a}$ and $\mathfrak{a}^{\prime}$ are simple and at least one of them principal.

By reference to $\mathrm{R}$ Theorems 38,59 , and 67 , we see immediately that Theorem 5 leads to the following characterization of the sets corresponding to prime ideals:

THEOREM 6. The set $\mathbb{E}(\mathfrak{p})$ corresponding to an arbitrary prime ideal $\mathfrak{p}$ in a Boolean ring $A$ is the open set $\mathbb{E}-\{\mathfrak{p}\}$; the ideal $p$ is normal, and hence semiprincipal, if and only if $\mathfrak{p}$ is an isolated point in the Boolean space $\mathbb{E}=\mathbb{E}(A)$.

Using Theorem 5 (3), we now obtain an important result.

THEOREM 7. The representatives of Boolean rings $B$ with the properties

(1) $B$ is isomorphic to a subring $\mathfrak{B}$ of the ring $\subseteq$ of all simple ideals in a Boolean ring $A$;

(2) if $\mathfrak{S}$ is an ideal in $\mathfrak{B}$, the relations $\mathrm{S}_{\mathfrak{a} \in \mathfrak{S}} \mathfrak{a}=\mathfrak{e}$ and $\mathfrak{S}=\mathfrak{B}$ are equivalent; are characterized topologically as those Boolean spaces which are continuous images of an arbitrary representative of $A$. In particular, the representatives of Boolean rings $B$ with the following properties:

(1) $B$ is isomorphic to a subring $\mathfrak{b}$ of a Boolean ring $A$ with unit $e$;

(2) $e$ is an element of $\mathfrak{b}$;

are characterized topologically as the totally-disconnected continuous images of an arbitrary representative of $A$.

By Theorem 4 we may restrict our attention to the subrings $B$ and the space $\mathbb{E}=\mathbb{E}(A)$ in establishing the first part of the present theorem. Let $\mathfrak{A}$ be a Boolean space which is a continuous image of $\&(A)$ by virtue of a correspondence $\mathbb{B}=f(\mathfrak{p})$. Then there exists a Boolean ring $B$ such that $\mathfrak{A}=\mathfrak{A}(B)$; and, if $\mathfrak{F}$ is any bicompact open set in $\mathfrak{A}$, its antecedent $f^{-1}(\mathfrak{F})$ is both open and closed in $(E$. By Theorem 5 (3) there exists a simple ideal in $A$ such that $f^{-1}(\mathfrak{F})=\mathfrak{F}(\mathfrak{a})$. The algebras with the classes $\mathfrak{F}, f^{-1}(\mathfrak{F})$ as elements are isomorphic to each other and to $B$, while the associated ideals $a$ constitute a subring of $\mathfrak{S}$ also isomorphic to $B$. This subring we designate as $\mathfrak{B}$. We can now write $\mathfrak{A}=\mathfrak{A}(\mathfrak{B})$ by virtue of the isomorphism between $B$ and $\mathfrak{B}$. If $\mathfrak{C}$ is any ideal in $\mathfrak{B}$, it is represented by an open set $\mathfrak{A}(\mathfrak{S})$ in $\mathfrak{A}$. It is evident that $f^{-1}(\mathfrak{A}(\mathfrak{S}))=\sum_{\mathfrak{a} \in \mathbb{S}} \mathfrak{E}(\mathfrak{a})=\mathfrak{F}\left(S_{\mathfrak{a} \in \mathbb{E}} \mathfrak{a}\right)$ and hence that the relations $\mathfrak{S}=\mathfrak{B}, \mathfrak{A}(\mathfrak{S})=\mathfrak{A}$, and $S_{\mathfrak{a} \in \mathbb{E}} \mathfrak{a}=\mathfrak{e}$ are equivalent. On the other hand, let $\mathfrak{B}$ be a Boolean ring with the properties (1) and (2). The elements $\mathfrak{a}$ of $\mathfrak{B}$ can then be represented in two ways: as the bicompact open subsets $\mathfrak{A}(\mathfrak{a})$ of a representative $\mathfrak{A}=\mathfrak{A}(\mathfrak{B})$, and as certain open-and-closed sets $\mathbb{E}(\mathfrak{a}), \mathfrak{a} \in \mathfrak{B} \subset \mathfrak{S}$, in $\mathbb{E}=\mathbb{E}(A)$. The corre- 
spondence $\mathfrak{E}(\mathfrak{a}) \leftrightarrow \rightarrow \mathfrak{A}(\mathfrak{a}), \mathfrak{a} \in \mathfrak{B}$, then induces a point correspondence between $\mathbb{E}$ and $\mathfrak{A}$. If $(\mathfrak{z}, \mathfrak{p})$ is a pair of points $\mathbb{z}$ and $\mathfrak{p}$ in $\mathfrak{A}$ and $\mathbb{E}$ respectively such that the relations $\mathfrak{A}(\mathfrak{a}) \subset \mathfrak{A}-\{\mathfrak{b}\}$ and $\mathfrak{E}(\mathfrak{a}) \subset \mathfrak{E}-\{\mathfrak{p}\}$ are either both true or both false whatever the element $\mathfrak{a}$ in $\mathfrak{B}$, we set $\mathfrak{z}=f(\mathfrak{p})$. We see that every $\mathbb{z}$ in $\mathfrak{A}$ belongs to at least one such pair: for the relation $\mathfrak{A}(\mathfrak{a}) \subset \mathfrak{A}-\{\mathfrak{z}\}$ determines a prime ideal $\mathfrak{B}$ in $\mathfrak{B}$; and, by hypothesis, the relation $\mathfrak{B} \neq \mathfrak{B}$ implies $\sum_{\mathfrak{a} \in \mathfrak{B}} \mathfrak{F}(\mathfrak{a})=\mathfrak{F}\left(\mathrm{S}_{\mathfrak{a} \in \mathfrak{B}} \mathfrak{a}\right) \neq \mathfrak{F}(\mathfrak{e})=\mathfrak{E}$. Furthermore we see that each $\mathfrak{p}$ belongs to exactly one such pair: for those elements $\mathfrak{a}$ in $\mathfrak{B}$ which satisfy the equivalent relations $\mathfrak{a} \subset \mathfrak{p}, \mathfrak{E}(\mathfrak{a}) \subset \mathfrak{E}(\mathfrak{p})$ constitute a prime ideal $\mathfrak{B}$ in $\mathfrak{B}$ in accordance with $\mathrm{R}$ Theorems 36 and 41 ; and there is then exactly one point 8 in $\mathfrak{A}$ such that $\sum_{\mathfrak{a} \in \mathfrak{B}} \mathfrak{A}(\mathfrak{a}) \subset \mathfrak{A}-\{\mathfrak{z}\}$. The relation $\mathbb{z}=f(\mathfrak{p})$ thus sets $\mathfrak{A}$ in univocal correspondence with $\mathfrak{E}$. Since the sets $\mathfrak{A}(\mathfrak{a})$ constitute a basis in $\mathfrak{A}$ and the sets $\mathfrak{E}(\mathfrak{a})$ are open in $\mathbb{E}$, it is sufficient in proving that $f(\mathfrak{p})$ is continuous, to show that $f^{-1}(\mathfrak{A}(\mathfrak{a}))=\mathfrak{E}(\mathfrak{a})$ for every $\mathfrak{a}$ in $\mathfrak{B}$. If $\mathfrak{a}$ is fixed, the points $\mathbb{z}$ and $\mathfrak{p}$ specified by the relations $f(\mathfrak{p})=\mathbb{z} \in \mathfrak{A}(\mathfrak{a})$ are by definition precisely those for which the relations $\mathfrak{A}(\mathfrak{a}) \subset \mathfrak{A}-\{\mathfrak{z}\}, \mathfrak{E}(\mathfrak{a}) \subset \mathfrak{E}-\{\mathfrak{p}\}$ are both false; and the desired relation $f^{-1}(\mathfrak{A}(\mathfrak{a}))=\mathfrak{F}(\mathfrak{a})$ is thus established.

The second part of the theorem follows by specialization of what has just been proved. If $A$ has a unit $e$, then $\subseteq$ is isomorphic to $A$ by $\mathrm{R}$ Theorems 25, 30 , and 31 . Hence the rings $\mathfrak{B}$ with the properties (1) and (2) of the first part of the theorem can be replaced by the subrings $\mathfrak{b}$ of $A$ which contain $e$ : for every subring of $\subseteq$ is isomorphic to a subring of $A$; and property (2), when expressed in terms of the generating elements of the principal ideals involved, asserts the equivalence of the relations $S_{a \mathrm{aec}} a=e$ and $\mathfrak{c}=\mathfrak{b}$ for an arbitrary ideal $\mathfrak{c}$ in $\mathfrak{b}$, and therefore degenerates, in accordance with $R$ Theorem 17, into the simpler condition $e \in \mathfrak{b}$. Theorems 1 and 3 now permit us to assert the second part of the present theorem.

As a final illustration of the connections between algebra and topology developed in the preceding theorems, we may comment informally on two distinct algebraic problems. First let us consider the determination of the ideal product of a non-void class $\mathfrak{A}$ of prime ideals $\mathfrak{p}$ in a Boolean ring $A$. It is clear that the complete solution of this problem can be given in the following topological terms: if $\mathfrak{a}=\prod_{\text {pere }} \mathfrak{p}$, then $\mathbb{E}(\mathfrak{a})=\left[\prod_{\text {pert }} \mathfrak{E}(\mathfrak{p})\right]^{\prime-^{\prime}}=\left[\sum_{\text {pert }}\{\mathfrak{p}\}\right]^{-\prime}$. Then let us consider the problem of representative elements in the modular classes of a Boolean ring $A$, recently discussed by v. Neumann and the writer.* In topological terms, this problem can now be rephrased as follows: if $\mathfrak{F}$ is any closed subset of a Boolean space $\mathfrak{B}$, it is required to determine in each class of sets intersecting $\mathfrak{F}$ in a fixed bicompact open subset of that

* J. v. Neumann and M. H. Stone, Fundamenta Mathematicae, vol. 25 (1935), pp. 353-378. 
subspace a representative member is so that (1) \& is a bicompact open subset of $\mathfrak{B},(2)$ the Boolean rings generated by the sets $B$ and $\mathscr{S} \mathfrak{F}$ respectively are isomorphic. This problem is evidently one which involves the position of $\mathfrak{F}$ in $\mathfrak{B}$; it has a solution if and only if $\mathfrak{F}$ has a special location in $\mathfrak{B}$.

3. Universal Boolean spaces and rings. In view of the relations between Boolean spaces and Boolean rings, it is of some interest to consider the algebraic significance of the facts concerning the imbedding of locallybicompact $H$-spaces in bicompact $H$-spaces. We shall consider first the specialization of the theorem to the effect that a locally-bicompact $H$-space can be imbedded in a bicompact $H$-space by the adjunction of a single point.* We have

THEOREM 8. The non-bicompact Boolean spaces are characterized topologically as the non-closed open subsets of bicompact Boolean spaces; in particular, every non-bicompact Boolean space can be converted by the adjunction of a single non-isolated point (in an essentially unique way) into a bicompact Boolean space. Accordingly, the Boolean rings without unit are characterized algebraically as the non-principal ideals in Boolean rings with unit; in particular, every Boolean ring without unit can be imbedded as a non-principal prime ideal in a Boolean ring with unit (in an essentially unique way).

We have already seen in Theorem 3 that every open subset of a Boolean space is a Boolean space. In a bicompact space a subset is closed if and only if it is bicompact. Hence we see that every non-closed open subset of a bicompact Boolean space is a non-bicompact Boolean space. The converse proposition, that every non-bicompact Boolean space is topologically equivalent to such a subset of some bicompact Boolean space, is a consequence of the assertion concerning the possibility of adjoining a point so as to make the resulting space bicompact. We shall not give a direct topological proof of this assertion, although one could easily be given on the basis of the reference made above to $\mathrm{AH}$. Instead, we deduce it from algebraic considerations. In $\mathrm{R}$ Theorems 1 and 37 we have already established the final algebraic statement of the present theorem: every Boolean ring without unit can be imbedded as a non-principal prime ideal in a Boolean ring with unit, which is uniquely determined if isomorphic systems be regarded as identical. Interpreting this fact topologically in accordance with Theorems 4 and 6, we see that every non-bicompact Boolean space is topologically equivalent to an open set obtained from an appropriate bicompact Boolean space by the removal of a single non-isolated point; and we see also that the imbedding space is uniquely determined if equivalent spaces be regarded as identical.

${ }^{*}$ AH, p. 93, Satz XIV. 
Thus, having established all statements of the present theorem except the general algebraic one, we are in a position to prove the latter by a translation of topological facts into algebraic terms. We omit the details.

We pass now to the consideration of a fundamental theorem of Tychonoff, ${ }^{*}$ to the effect that every completely regular space can be imbedded in a certain universal bicompact $H$-space of the same character. This result suggests a corresponding specialization to the case of Boolean spaces, together with its algebraic interpretation. In formulating such a special theorem, we may disregard the trivial case of finite Boolean spaces: each of them consists of a finite number of discrete points. We therefore state our results in terms of Boolean spaces of infinite character. We have first the following theorem.

THEOREM 9. Let $\mathrm{c}$ be an arbitrary infinite cardinal number; let $\mathrm{A}$ be an arbitrary class of cardinal number $\mathrm{c}$, for example, the class of all ordinal numbers preceding some suitable (even the first suitable) ordinal number $\omega$; let $\mathfrak{B}_{\mathrm{c}}$ be the class of all characteristic functions $8=8(\alpha)$ defined over $\mathrm{A}$ (for each $\alpha$ in $\mathrm{A}$, either $\mathfrak{B}(\alpha)=0$ or $\mathfrak{B}(\alpha)=1)$; and let $A_{\mathfrak{c}}$ be the class of all sets in $\mathfrak{B}_{\mathfrak{c}}$ generated from the special sets $\mathfrak{U}_{\alpha}, \mathfrak{U}_{\alpha}^{\prime}$, where $\mathfrak{H}_{\alpha}$ contains all \& for which $\mathfrak{B}(\alpha)=0$, by the formation of finite unions and intersections. By the assignment of each non-void set belonging to $A_{\mathfrak{c}}$ as a neighborhood of every one of its points, $\mathfrak{B}_{\mathfrak{c}}$ becomes a bicompact Boolean space of character $c$. The system $A_{\mathrm{c}}$ is a Boolean ring with the set $\mathfrak{B}_{\mathfrak{c}}$ as its unit, with $\mathrm{c}$ as its cardinal number, and with $\mathfrak{B}_{\mathfrak{c}}$ as one of its representative Boolean spaces.

In view of Theorem 1, we can establish the present theorem by showing that $A_{\mathfrak{c}}$ is a perfect reduced algebra of classes in $\mathfrak{B}_{\mathfrak{c}}$. From $\mathrm{R}$ Theorem 14 , we see that $A_{\mathrm{c}}$ is a Boolean ring with classes as elements and the particular class $\mathfrak{B}_{\mathrm{c}}$ as its unit; and, since the cardinal number of the class of all sets $\mathfrak{U}_{\alpha}$ is precisely $\mathrm{c}$, we can easily calculate the cardinal number of $A_{\mathrm{c}}$ as equal to $\mathrm{c}$. This algebra of classes is reduced in the sense of $\mathrm{R}$ Definition 10: for it contains the class $\mathfrak{B}_{\mathfrak{c}}$; and an arbitrary point $\mathbb{B}_{0}=\mathbb{B}_{0}(\alpha)$ in $\mathfrak{B}_{\mathfrak{c}}$ is obviously the sole point common to the sets $\mathfrak{U}_{\alpha}$ where $\alpha$ is such that $\mathbb{B}_{0}(\alpha)=0$ and the sets $\mathfrak{U}_{\alpha}^{\prime}$ where $\alpha$ is such that $\varepsilon_{0}(\alpha)=1$. To show that $A_{\mathrm{c}}$ is a perfect algebra of classes, we begin by considering an arbitrary prime ideal $\mathfrak{p}$ in $A_{\mathfrak{c}}$ and defining a corresponding point $\mathfrak{B}_{\mathfrak{p}}$ in $\mathfrak{B}_{\mathfrak{c}}$ by setting $\mathfrak{B}_{\mathfrak{p}}(\alpha)=0$ or $\mathfrak{B}_{\mathfrak{p}}(\alpha)=1$ according as $\mathfrak{U}_{\alpha}^{\prime} \in \mathfrak{p}$ or $\mathfrak{U}_{\alpha} \in \mathfrak{p}$. We can then prove that no set $\mathfrak{U}$ belonging to $\mathfrak{p}$ contains the point $\mathfrak{B}_{\mathfrak{p}}$. If $\mathfrak{U}$ is in $\mathfrak{p}$, its complement $\mathfrak{U}^{\prime}$ is not a member of $\mathfrak{p}$; since $\mathfrak{U}^{\prime}$ can be represented as a finite union of terms $\mathfrak{U}_{\alpha_{1}} \cdots \mathfrak{U}_{\alpha_{m}} \mathfrak{U}_{\beta_{1}{ }^{\prime}} \cdots \mathfrak{U}_{\beta_{n}}{ }^{\prime}$, where $\alpha_{1}, \cdots, \alpha_{m}$, $\beta_{1}, \cdots, \beta_{n}$ are in A and either the $\alpha$ 's or the $\beta$ 's may be absent, at least one

\footnotetext{
* Tychonoff, Mathematische Annalen, vol. 102 (1930), pp. 544-561.
} 
of these terms, and indeed every factor of that term, must be a non-member of $\mathfrak{p}$ by virtue of $\mathrm{R}$ Theorem 34 . Thus the relation $\mathfrak{U}_{\alpha_{1}} \cdots \mathfrak{u}_{\alpha_{m}} \mathfrak{U}_{\beta_{1}^{\prime}} \cdots \mathfrak{u}_{\beta_{n}^{\prime}} \subset \mathfrak{U}^{\prime}$ can be satisfied by appropriate choice of $\mathfrak{u}_{\alpha_{1}{ }_{1}}, \cdots, \mathfrak{u}_{\alpha_{m}{ }^{\prime}}, \mathfrak{u}_{\beta_{1}}, \cdots, \mathfrak{u}_{\beta_{n}}$ in $\mathfrak{p}$. The equations $\mathbb{B}_{\mathfrak{p}}\left(\alpha_{1}\right)=\cdots=\mathbb{B}_{\mathfrak{p}}\left(\alpha_{m}\right)=0, \mathbb{B}_{\mathfrak{p}}\left(\beta_{1}\right)=\cdots=\mathbb{B}_{\mathfrak{p}}\left(\beta_{n}\right)=1$ now show that $\mathbb{B}_{\mathfrak{p}}$ is contained in $\mathfrak{u}_{\alpha_{1}} \cdots \mathfrak{u}_{\alpha_{m}} \mathfrak{u}_{\beta_{1}} \cdots \mathfrak{u}_{\beta_{n}}{ }^{\prime}$ and in $\mathfrak{u}^{\prime}$ but not in $\mathfrak{u}$. From $\mathrm{R}$ Theorems 59 and 66 it follows therefore that $A_{\mathrm{c}}$ is a perfect reduced algebra of classes, and is indeed a perfect representation of itself. Theorem 1 shows at once that $\mathfrak{B}_{\mathfrak{c}}$ is a bicompact Boolean space of character $\mathrm{c}$ which is a representative of $A_{\mathrm{c}}$ in accordance with Definitions 1 and 2.

TheOREM 10. Every Boolean space of character not exceeding $\mathrm{c}$ is topologically equivalent to a subspace of the space $\mathfrak{B}_{\mathrm{c}}$ of Theorem 9; and every Boolean ring with unit which has cardinal number not exceeding $\mathrm{c}$ is a homomorph of the ring $A_{\mathrm{c}}$.

We may obviously restrict attention to a Boolean ring $A$ and its particular representative Boolean space $\mathbb{E}=\mathbb{E}(A)$. We may, if we wish, include the case of finite rings. For infinite rings, the cardinal number of $A$ is equal to the character of $\mathbb{E}$. Hence the assumption that either the cardinal number of $A$ or the character of $\mathbb{E}(A)$ does not exceed $\mathfrak{c}$ permits us to choose in the class $\mathrm{A}$ of Theorem 9 a subset $\Gamma$ with the same cardinal number as $A$, and to set up a biunivocal correspondence between $A$ and $\Gamma$, designating the element of $A$ associated with the element $\alpha$ in $\Gamma$ by $a_{\alpha}$. In case the cardinal number of $A$ is equal to $\mathrm{c}$ we may take $\Gamma=\mathrm{A}$ or $\Gamma \neq \mathrm{A}$ as we wish; in all other cases we must take $\Gamma \neq A$. If now $p$ is any point in $\mathcal{E}$, that is, if $\mathfrak{p}$ is any prime ideal in $A$, we define a corresponding point $\mathfrak{B}_{\mathfrak{p}}$ in $\mathfrak{B}_{\mathfrak{c}}$ by putting $\mathfrak{B}_{\mathfrak{p}}(\alpha)=0$ if $\alpha \epsilon \mathrm{A}-\Gamma, \mathbb{B}_{\mathfrak{p}}(\alpha)=0$ if $\alpha \epsilon \Gamma$ and $a_{\alpha} \notin \mathfrak{p}$, and $\mathbb{B}_{\mathfrak{p}}(\alpha)=1$ if $\alpha \epsilon \Gamma$ and $a_{\alpha} \in \mathfrak{p}$. We recall that $\mathfrak{p} \in \mathbb{E}\left(a_{\alpha}\right)$ if and only if $a_{\alpha} \notin \mathfrak{p}$. The class of all points $\mathbb{B}_{\mathfrak{p}}$ thus obtained will be denoted by $\mathfrak{B}_{\mathrm{c}}(A)$. It is evident that the correspondence between $\mathbb{E}$ and $\mathfrak{B}_{\mathfrak{c}}(A)$ is biunivocal. In order that a subset of $\mathfrak{B}_{\mathfrak{c}}(A)$ be the image of $\mathbb{E}\left(a_{\beta}\right)$, it is necessary and sufficient that its points $\mathfrak{B}_{\mathfrak{p}}$ have indices $\mathfrak{p}$ such that $p \in \mathbb{E}\left(a_{\beta}\right)$ or, equivalently, such that ${\boldsymbol{B}_{p}}_{\mathfrak{p}}(\beta)=0$. Thus the images of the sets $\mathbb{E}\left(a_{\beta}\right)$ in $\mathbb{E}$ are precisely the sets $\mathfrak{u}_{\beta} \mathfrak{B}_{\mathfrak{c}}(A)$, where $\beta \epsilon \Gamma$. It is evident that $\alpha \in \mathrm{A}-\Gamma$ implies $\mathfrak{U}_{\alpha} \supset \mathfrak{B}_{\mathfrak{c}}(A)$. Now the intersections of the sets in $A_{\mathfrak{c}}$ with $\mathfrak{B}_{\mathfrak{c}}(A)$ constitute a basis in $\mathfrak{B}_{\mathfrak{c}}(A)$; indeed, by the definition of the class $A_{\mathfrak{c}}$, we may restrict attention to the sets $\mathfrak{U}_{\alpha_{1}} \cdots \mathfrak{u}_{\alpha_{m}} \mathfrak{u}_{\beta_{1}^{\prime}} \cdots \mathfrak{u}_{\beta_{n}}{ }^{\prime}$ which have points in common with $\mathfrak{B}_{\mathfrak{c}}(A)$, it being understood that either the $\alpha$ 's or the $\beta$ 's may be absent. Under the indicated restriction we may suppose, by virtue of the previous observations, that the indices $\alpha_{1}, \cdots, \alpha_{m}, \beta_{1}, \cdots, \beta_{n}$ belong to $\Gamma$. The sets $\mathfrak{u}_{\alpha_{1}} \cdots \mathfrak{U}_{\alpha_{m}} \mathfrak{U}_{\beta_{1}^{\prime}} \cdots \mathfrak{u}_{\beta_{n}^{\prime}}^{\prime} \mathfrak{B}_{\mathfrak{c}}(A)$ are then precisely the images of the sets $\mathbb{E}\left(a_{\alpha_{1}}\right) \ldots \mathbb{E}\left(a_{\alpha_{m}}\right) \mathbb{F}^{\prime}\left(a_{\beta_{1}}\right) \ldots \mathbb{F}^{\prime}\left(a_{\beta_{n}}\right)=\mathbb{E}\left(a_{\gamma}\right) \mathbb{F}^{\prime}\left(a_{\delta}\right)$ in $\mathbb{E}$, 
where $a_{\gamma}=a_{\alpha_{1}} \cdots a_{\alpha_{m}}$ and $a_{\delta}=a_{\beta_{1}} \vee \cdots \vee a_{\beta_{n}}$. Since the latter sets obviously constitute a basis in $₹$, we see that the correspondence set up between $\&$ and $\mathfrak{B}_{\mathfrak{c}}(A)$ is a topological equivalence. When $A$ has a unit, $\mathbb{E}$ and $\mathfrak{B}_{\mathfrak{c}}(A)$ are bicompact; and $\mathfrak{B}_{\mathfrak{c}}(A)$ is therefore closed in $\mathfrak{B}_{\mathfrak{c}}$. By Theorem 4 it follows that $A$ is a homomorph of $A_{\mathrm{c}}$.

The theorem just proved is the desired specialization of the theorem of Tychonoff. From it we can obtain further useful facts.

Theorem 11. The Boolean spaces $\mathfrak{B}_{\mathfrak{c}}-\{\mathfrak{z}\}$ obtained from $\mathfrak{B}_{\mathrm{c}}$ by the suppression of a single point 8 are topologically equivalent and all non-bicompact; the prime ideals in the Boolean ring $A_{\mathrm{c}}$ are isomorphic Boolean rings without unit. If any common isomorph of the prime ideals in $A_{\mathrm{c}}$ be denoted by $A_{\mathrm{c}}^{*}$, then every Boolean ring of cardinal number not exceeding $\mathrm{c}$ is a homomorph of $A_{\mathfrak{c}}^{*}$.

The relations $\mathfrak{B}^{\prime}=\mathbb{z}+\mathbb{z}_{0}(\bmod 2), \mathbb{z}_{0}=z_{2}-z_{1}(\bmod 2)$ obviously set up a biunivocal correspondence of $\mathfrak{B}_{c}$ with itself, carrying $\mathbb{B}_{1}$ into $\mathbb{B}^{\prime}=\mathbb{B}_{2}$. It is evident that the image of the set $\mathfrak{U}_{\alpha}$ under this correspondence is the set specified by $\mathfrak{B}^{\prime}(\alpha)=\mathbb{s}_{0}(\alpha)$ and hence that the image is either $\mathfrak{u}_{\alpha}$ or $\mathfrak{U}_{\alpha}^{\prime}$ according as $\dot{B}_{0}(\alpha)=0$ or $\dot{B}_{0}(\alpha)=1$. It follows readily that the correspondence transforms $A_{\mathrm{c}}$ into itself and is therefore a topological transformation of $\mathfrak{B}_{\mathrm{c}}$ into itself. In consequence, the spaces $\mathfrak{B}_{\mathfrak{c}}-\left\{\mathbb{B}_{1}\right\}$ and $\mathfrak{B}_{\mathfrak{c}}-\left\{\mathscr{B}_{2}\right\}$ are topologically equivalent. It is clear that no point of $\mathfrak{B}_{\mathfrak{c}}$ is isolated: this can be verified directly; or can be proved by noting that, if one point were isolated, then every point would be isolated in accordance with the fact that the topological group of $\mathfrak{B}_{\mathrm{c}}$ is transitive, but in contradiction with the fact that $\mathfrak{B}_{\mathrm{c}}$ is bicompact. We see therefore that every space $\mathfrak{B}_{\mathfrak{c}}-\{\mathbb{B}\}$ is a non-bicompact Boolean space. By Theorems 4 and 6, these results imply that the prime ideals in $A_{\mathrm{c}}$ have the asserted properties. If we recall the relations between prime ideals and atomic elements described in $\mathrm{R}$ Theorem 38, we see in particular that $A_{\mathrm{c}}$ contains no atomic element and no normal prime ideal.

It is now easily verified that every Boolean ring $A$ of cardinal number not exceeding $\mathrm{c}$ is a homomorph of $A_{\mathrm{c}}^{*}$. We first adjoin a unit to $A$, if necessary, obtaining a Boolean ring $A^{*}$. This adjunction can be effected by means of $\mathrm{R}$ Theorem 1 . It is easily seen by reference to the construction given there that the cardinal number of $A^{*}$ likewise does not exceed c. We now proceed, as in the proof of Theorem 10 , to construct a representative $\mathfrak{B}_{\mathfrak{c}}\left(A^{*}\right)$ of $A^{*}$, taking $\Gamma$ different from $A$ so that $\mathfrak{B}_{\mathrm{c}}\left(A^{*}\right)$ is a proper subset of $\mathfrak{B}_{\mathrm{c}}$. When $A$ has a unit, we have $A=A^{*}$ and know that $\mathfrak{B}_{\mathrm{c}}\left(A^{*}\right)$ is a closed subset of $\mathfrak{B}_{\mathrm{c}}$. Thus, if we choose 8 as a point in $\mathfrak{B}_{\mathfrak{c}}^{\prime}\left(A^{*}\right)$, the space $\mathfrak{B}_{\mathfrak{c}}-\{\xi\}$ contains $\mathfrak{B}_{\mathfrak{c}}\left(A^{*}\right)$ as a closed subset. In this case, $A=A^{*}$ is a homomorph of $A_{\mathrm{c}}^{*}$ in accordance with Theorem 4 . When $A$ has no unit, $A$ is a prime ideal in $A^{*}$ by $\mathrm{R}$ The- 
orem 37. It follows that a representative $\mathfrak{B}_{\mathrm{c}}(A)$ of $A$ can be found by removing an appropriate point $\&$ from the space $\mathfrak{B}_{\mathfrak{c}}\left(A^{*}\right)$. Since we have $\mathfrak{B}_{\mathfrak{c}}(A)=\mathfrak{B}_{\mathfrak{c}}\left(A^{*}\right)\left(\mathfrak{B}_{\mathfrak{c}}-\{\mathfrak{B}\}\right)$, the set $\mathfrak{B}_{\mathfrak{c}}(A)$ is closed in the space $\mathfrak{B}_{\mathfrak{c}}-\{\mathfrak{B}\}$. Hence in this case also, $A$ is a homomorph of $A_{\mathfrak{c}}^{*}$.

Just as the Boolean space $\mathfrak{B}_{\mathfrak{c}}$ is a universal Boolean space in the sense that in it can be imbedded every Boolean space of sufficiently small character, so the Boolean rings $A_{\mathrm{c}}$ and $A_{\mathrm{c}}^{*}$ are universal Boolean rings in the sense that their homomorphs exhaust all Boolean rings of cardinal number not exceeding c, due regard being paid in the case of $A_{\mathfrak{c}}$ to the condition that the homomorphs of a ring with unit all possess units. If we recall that the homomorphs of a Boolean ring can be obtained through the replacement of the fundamental equality by the various congruences in the ring, we are led to formulate the following algebraic characterization of the universal rings $A_{\mathrm{c}}$ and $A_{\mathrm{c}}^{*}$ :

Theorem 12. The Boolean rings $A_{\mathrm{c}}$ and $A_{\mathrm{c}}^{*}$ are isomorphic respectively to the free Boolean rings with and without unit generated by $c$ elements.

The free Boolean ring without unit generated by $c$ elements is obtained by forming all abstract or symbolic polynomials in these elements and then introducing the weakest possible relation of equality consistent with the postulates for a Boolean ring without unit. In accordance with this procedure the symbolic polynomials $a+a$, where $a$ is a generating element, must all be equated; and we can denote some one of them by 0 . The elements of the free ring which are equal to 0 then have in common the properties of a zero-element. If we make use of the postulates for Boolean rings, we can describe the introduction of the equality between symbolic polynomials in a more precise way: the desired equality is determined uniquely by the property that a symbolic polynomial is equated to 0 if and only if the algebraic laws expressed or implied in the fundamental postulates for a Boolean ring without unit reduce it formally to 0 . We thus see that the free ring can be characterized as follows: it is a Boolean ring without unit; it contains a subset of cardinal number $c$ which generates the entire ring; and the elements of this subset satisfy no algebraic relation which is not a Boolean identity.

The free Boolean ring with unit generated by $c$ elements is obtained by forming the free ring without unit generated by the given elements and then adjoining a unit by the construction of $\mathrm{R}$ Theorem 1 . Thus this free ring can be characterized as follows: it is a Boolean ring with unit; it contains a subset of cardinal number $c$ which, together with the unit, generates the entire ring; and the elements of this subset satisfy no algebraic relation which is not a Boolean identity. 
In order to apply the given characterizations of free rings, we need a criterion to determine whether or not the elements of a subclass $a$ of a Boolean ring $A$ are free from non-identical algebraic relations. Such a criterion can be established as follows: in order that the elements of $\mathfrak{a}$ shall satisfy no algebraic relation which is not a Boolean identity, it is necessary and sufficient that no relation $a_{1} \cdots a_{m}<b_{1} \vee \cdots \vee b_{n}, m \geqq 1, n \geqq 1$ shall hold between distinct elements $a_{1}, \cdots, a_{m}, b_{1}, \cdots, b_{n}$ in a. We first suppose that $A$ has a unit. Then any non-identical algebraic relation connecting distinct elements of $a$ can be reduced to the form $d_{1} \vee \cdots \vee d_{p}=0$, where each element $d$ is expressed in terms of distinct elements of $a$ by a relation $d=a_{1} \cdots a_{m} b_{1}^{\prime} \cdots b_{n}^{\prime}$. The given relation therefore implies $d_{1}=\cdots=d_{p}=0$ and hence $a_{1} \cdots a_{m} b_{1}^{\prime} \cdots b_{n}^{\prime}=0$. We may obviously suppose that $m \geqq 1, n \geqq 1$ : for, if $b_{1}^{\prime} \cdots b_{n}^{\prime}=0$ or $a_{1} \cdots a_{m}=0$, the missing elements $a_{1}, \cdots, a_{m}$ or $b_{1}^{\prime}, \cdots, b_{n}^{\prime}$ can be inserted arbitrarily. Thus we have shown that any nonidentical algebraic relation connecting distinct elements of $a$ implies a relation $a_{1} \cdots a_{m} b_{1}^{\prime} \cdots b_{n}{ }^{\prime}=0$ or the equivalent relation $a_{1} \cdots a_{m}<b_{1} \vee \cdots \vee b_{n}$, $m \geqq 1, n \geqq 1$, connecting distinct elements of a. In case $A$ has no unit, we adjoin one by the construction of $\mathrm{R}$ Theorem 1 and repeat the argument just given. It is thus evident in all cases that $\mathfrak{a}$ is free from non-identical algebraic relations if and only if no relation $a_{1} \cdots a_{m}<b_{1} \vee \cdots \vee b_{n}$ holds between distinct elements of $\mathfrak{a}$.

The Boolean ring $A_{\mathfrak{c}}$ is evidently generated by the class of all sets $\mathfrak{U}_{\alpha}$ together with the unit $\mathfrak{B}_{\mathfrak{c}}$. This class of sets obviously has cardinal number $\mathrm{c}$. We now observe that the relation $\mathfrak{H}_{\alpha_{1}} \cdots \mathfrak{U}_{\alpha_{m}} \subset \mathfrak{U}_{\beta_{1}} \cup \cdots \cup \mathfrak{U}_{\beta_{n}}, m \geqq 1$, $n \geqq 1$, means that every characteristic function $8=8(\alpha)$ with the property $\mathbb{B}\left(\alpha_{1}\right)=\cdots=\mathbb{8}\left(\alpha_{m}\right)=0$ has also at least one of the properties $\mathbb{B}\left(\beta_{1}\right)=0, \cdots$, $8\left(\beta_{n}\right)=0$. Obviously, then, no such relation can hold when the indices $\alpha_{1}, \cdots, \alpha_{m}, \beta_{1}, \cdots, \beta_{n}$ are distinct. It follows that $A_{c}$ is isomorphic to the free Boolean ring with unit generated by $c$ elements.

The Boolean ring $A_{\mathrm{c}}^{*}$ may be regarded as that prime ideal in $A_{\mathrm{c}}$ which contains every set $\mathfrak{u}_{\alpha}$. In fact, we can determine a prime ideal in $A_{\mathfrak{c}}$ by considering those sets in $A_{\mathfrak{c}}$ which are contained in $\mathfrak{B}_{\mathfrak{c}}-\{\mathfrak{z}\}$, where $\mathbb{B}(\alpha)=1$ for every $\alpha$. It is evident that this prime ideal contains every set $\mathfrak{u}_{\alpha}$. Since $A_{\mathfrak{c}}^{*}$ is isomorphic to any prime ideal in $A_{\mathfrak{c}}$, we may identify it with the one just determined. It is then evident that the ring $A_{\mathrm{c}}^{*}$ is generated by the class of all sets $\mathfrak{u}_{\alpha}$. As in the preceding paragraph, it follows that $A_{\mathfrak{c}}^{*}$ is isomorphic to the free Boolean ring without unit generated by $c$ elements.

In conclusion, it is of some interest to remark upon the duality between the free Boolean ring with unit generated by $c$ elements and the Boolean ring of all subclasses of a fixed class of cardinal number $c$ which emerges 
from the previous discussion. Since the characteristic functions $8(\alpha)$ constitute under multiplication and addition $(\bmod 2)$ a Boolean ring isomorphic to the ring of all subclasses of $\mathrm{A}$, our construction of the free ring $A_{\mathrm{c}}$ in terms of these characteristic functions may be regarded as exhibiting a dual relationship between the rings in question. This duality is in fact a special instance of that which holds between discrete abelian groups and the subgroups of toroidal groups:* for Boolean rings are abelian groups under addition and can be studied from that point of view. We shall not pursue this aspect of the subject further.

We shall close the present section with an examination of the special case $\mathbf{c}=\boldsymbol{\aleph}_{0}$. We obtain a result which explains in some measure the frequent occurrence of the Cantor discontinuum in various branches of mathematics.

Theorem 13. The Boolean space $\mathfrak{B}_{\mathfrak{c}}, \mathfrak{c}=\boldsymbol{\aleph}_{0}$, is topologically equivalent to the space $\mathfrak{D}$ known as the Cantor discontinuum.

$\mathfrak{D}$ may be described as the set of all real numbers $x$ given by developments $x=2 \sum_{\alpha=1}^{\infty} 3^{-\alpha} s(\alpha)$, where $s(k)=0$ or $s(k)=1$, with the usual metric topology. An important property of $\mathfrak{D}$ is the following: a sequence $\left\{x_{n}\right\}$ in $\mathfrak{D}$ converges if and only if $\left\{s_{n}(k)\right\}$ converges for each $k$; and the limit of $\left\{x_{n}\right\}$ is obtained as the element $x$ with development given by $s(k)=\lim _{n \rightarrow \infty} s_{n}(k), k=1,2$, $3, \cdots$. To prove this assertion, we consider the inequality

$$
\begin{aligned}
\left|2 \sum_{\alpha=1}^{N} 3^{-\alpha}\left(s_{m}(\alpha)-s_{n}(\alpha)\right)\right| & \leqq 2 \sum_{\alpha=N+1}^{\infty} 3^{-\alpha}\left|s_{m}(\alpha)-s_{n}(\alpha)\right|+\left|x_{m}-x_{n}\right| \\
& \leqq 3^{-N}+\left|x_{m}-x_{n}\right| .
\end{aligned}
$$

If $\left\{x_{n}\right\}$ is convergent and $N$ is the first integer such that $\left\{s_{n}(N)\right\}$ is not known to converge, this inequality becomes

$$
2 \cdot 3^{-N}\left|s_{m}(N)-s_{n}(N)\right| \leqq 3^{-N}+\left|x_{m}-x_{n}\right|
$$

for all sufficiently large indices $m$ and $n$. If we restrict $m$ and $n$ to be so great that $\left|x_{m}-x_{n}\right|<3^{-N}$, the inequality reduces to $\left|s_{m}(N)-s_{n}(N)\right|<1$. Hence we must have $s_{m}(N)=s_{n}(N)$ for all sufficiently large indices $m$ and $n$. Since this result establishes the convergence of $\left\{s_{n}(N)\right\}$, we can apply the principle of mathematical induction to conclude that $\left\{s_{n}(k)\right\}$ converges for every $k$. If we know that $s(k)=\lim _{n \rightarrow \infty} s_{n}(k)$ exists for every $k$, we see readily that the number $x=2 \sum_{\alpha=1}^{\infty} 3^{-\alpha} s(\alpha)$ has the property

$$
\left|x-x_{n}\right| \leqq 2 \sum_{\alpha=1}^{\infty} 3^{-\alpha}\left|s(\alpha)-s_{n}(\alpha)\right| \leqq 2 \sum_{\alpha=1}^{N} 3^{-\alpha}\left|s(\alpha)-s_{n}(\alpha)\right|+3^{-N}<\epsilon
$$

* Alexander and Zippin, Annals of Mathematics, (2), vol. 36 (1935), pp. 71-85. 
for $N>-\log \epsilon / \log 3, n>M=M(N)$. Combining these results in the obvious way, we obtain the proposition stated above. There are several useful consequences of this proposition. First,it shows that if $x$ and $x^{\prime}$ have developments given by $s(k)$ and $s^{\prime}(k)$ respectively, then $x=x^{\prime}$ implies $s(k)=s^{\prime}(k)$ for every $k$ : indeed, the sequence $x, x^{\prime}, x, x^{\prime}, \cdots$ is then convergent so that the sequence $s(k), s^{\prime}(k), s(k), s^{\prime}(k), \cdots$ must converge likewise, for every $k$. Secondly, it shows that the set of points $x$ obtained by fixing the values of $s(k)$ for $k=1, \cdots, N$ and leaving the values of $s(k)$ for $k \geqq N+1$ arbitrary is an open subset of $\mathfrak{D}$ : for the complement of this set in $\mathfrak{D}$ has the property that any convergent subsequence has a limit for which one of the developmentcoefficients $s(k), k=1, \cdots, N$, has a value different from the one prescribed; in other words, the complement of the given set is closed in $\mathfrak{D}$. Thirdly, it shows that $\mathfrak{D}$ is bicompact: for any convergent sequence in $\mathfrak{D}$ has a limit in $\mathfrak{D}$; and $\mathfrak{D}$ is thus a closed subset of the bicompact space consisting of the real numbers $x$, where $0 \leqq x \leqq 1$, with the usual topology. The correspondence given by $x \rightarrow \mathfrak{B}=s(k)$ is a biunivocal correspondence between $\mathfrak{D}$ and $\mathfrak{B}_{\mathfrak{c}}, \mathfrak{c}=\boldsymbol{N}_{0}$, by virtue of our first remark. The sets $\mathfrak{u}_{\alpha_{1}} \cdots \mathfrak{u}_{\alpha_{m}} \mathfrak{u}_{\beta_{1}}{ }^{\prime} \cdots \mathfrak{U}_{\beta_{n}}{ }^{\prime}$ where $m+n=N$ and $\left(\alpha_{1}, \cdots, \alpha_{m}, \beta_{1}, \cdots, \beta_{n}\right)$ is a permutation of $(1, \cdots, N)$ obviously constitute a basis in $\mathfrak{B}_{\mathfrak{c}}$. Their antecedents in $\mathfrak{D}$ are precisely the sets obtained by fixing the first $N$ coefficients in the development of $x$ in each of the $2^{N}$ possible ways; and, since these antecedents are open in $\mathfrak{D}$ by virtue of our second remark, the correspondence from $\mathfrak{D}$ to $\mathfrak{B}_{\mathfrak{c}}, \mathfrak{c}=\boldsymbol{\aleph}_{0}$ is continuous. Finally, since $\mathfrak{D}$ and $\mathfrak{B}_{\mathfrak{c}}, \mathfrak{c}=\boldsymbol{\aleph}_{0}$, are both bicompact $H$-spaces, the correspondence is necessarily bicontinuous; and $\mathfrak{D}$ and $\mathfrak{B}_{\mathfrak{c}}, \mathfrak{c}=\boldsymbol{N}_{0}$, are topologically equivalent.*

\section{Chapter II. Maps in Boolean spaces}

1. The general theory of maps. The general theory of maps deals with the problem of representing the points and properties of a given topological space by subsets and their properties in a second topological space. While we shall be concerned primarily with the study of such representations in Boolean spaces, we shall begin with an elementary discussion of quite general maps, introducing the terminology appropriate to the later theory.

The point of departure for the entire theory is the following result:

TheOREM 14. If $X$ is any non-void family of distinct, but not necessarily disjoint, non-void closed sets $\mathfrak{X}$ in a $T_{1}$-space $\mathfrak{S}$, then a topology $\dagger$ may be imposed on $X$ by the introduction of the following system of neighborhoods: each

* AH, p. 95, Satz III.

$\dagger$ Compare with $\mathrm{AH}, \mathrm{p}$. 66, where the sets $\mathfrak{X}$ are assumed to be disjoint and the topology is termed "weak" to distinguish it from a different topology introduced earlier on pp. 61-62. 
non-void subfamily of $X$ which is characterized as the class of all $\mathfrak{X}$ contained in a fixed open subset $\mathbb{S}$ of $\mathfrak{S}$ is assigned as a neighborhood of every one of its elements $\mathfrak{X}$. Under this topology, $X$ is a $T_{0}$-space. In order that $X$ be a $T_{1}$-space, it is necessary and sufficient that no member of $X$ contain another as a proper subset.

It is evident that the system of neighborhoods described in the theorem has the following properties: every "point" $\mathfrak{X}$ belongs to each of its neighborhoods; the intersection of any two neighborhoods of an arbitrary point is also a neighborhood of that point; if an arbitrary neighborhood contains a given point, it is a neighborhood of that point; and every point has at least one neighborhood. Furthermore, we can show that, if $\mathfrak{X}_{1}$ and $\mathfrak{X}_{2}$ are distinct points, then at least one of them has a neighborhood which does not contain the other. Since $\mathfrak{X}_{1}$ and $\mathfrak{X}_{2}$ are distinct as subsets of $\mathfrak{S}$, we may suppose our notation so chosen that $\mathfrak{X}_{2}$ contains a point $\mathbb{z}_{2}$ of $\subseteq$ which does not belong to $\mathfrak{X}_{1}$. Since $S$ is a $T_{1}$-space, every $\mathbb{Z}_{1}$ in $\mathfrak{X}_{1}$ is contained in an open set $\mathfrak{B}\left(\mathbb{B}_{1}\right)$ which does not contain $\mathbb{B}_{2}$; and the union of all the open sets $\mathbb{S}\left(\mathbb{B}_{1}\right), \mathbb{B}_{1} \in \mathfrak{X}_{1}$, is an open set $\mathbb{B}$ which contains $\mathfrak{X}_{1}$ but does not contain either $\mathbb{B}_{2}$ or the set $\mathfrak{X}_{2}$. It is then evident that the family specified by $\mathfrak{X} \subset \mathcal{B}$ is a neighborhood of $\mathfrak{X}_{1}$ which does not contain $\mathfrak{X}_{2}$. From this argument we see that, if neither of the distinct sets $\mathfrak{X}_{1}$ and $\mathfrak{X}_{2}$ contains the other, then each has, as a point in $\mathcal{X}$, a neighborhood which does not contain the other; and also that, if one contains the other, as a proper subset, then every one of its neighborhoods contains the second. These facts concerning the system of neighborhoods identify $\mathcal{X}$ as a $T_{0}$-space, and show that $\mathcal{X}$ is a $T_{1}$-space if and only if no set $\mathfrak{X}$ contains another as a proper subset.*

It is convenient, in view of further developments, to introduce a certain amount of descriptive terminology relating to the family $x$. We therefore give the following definitions:

Definition 3. If the union $\mathfrak{S}(X)$ of the sets belonging to $X$ contains a subset $\mathfrak{A}$ of $\mathfrak{S}$, then $X$ is said to cover $\mathfrak{A}$.

DEFINITION 4. If every non-void open set $\$$ in $\mathfrak{S}$ contains some member of $\mathcal{X}$ as a subset, then $X$ is said to be densely distributed in $\mathfrak{S}$.

Definition 5. If, whenever $\mathbb{B}$ is an open set in $\subseteq$ and $\mathfrak{X}_{0}$ is a member of $X$, the relation $\& \supset \mathfrak{X}_{0}$ implies the existence of an open set $\mathfrak{G}_{0}$ such that $\mathfrak{S}_{0} \supset \mathfrak{X}_{0}$ and $\mathfrak{X} \subset(S)$ whenever $\mathfrak{X} \mathfrak{S}_{0} \neq 0$, then $X$ is called a continuous family.

Definition 6. If $\mathrm{c}$ is the least cardinal number such that $\mathrm{c}+1$ is exceeded by the cardinal number of no set $\mathfrak{X}$ in $X$, then $c$ is called the index of the family $X$.

* AH, pp. 58-59. 
If some set $\mathfrak{X}$ in $X$ has cardinal number $\mathrm{c}+1$, the index is said to be attained.

We proceed now to give the principal definitions dealing with the general concept of a map.

Definition 7. If $\Re$ is a topological space equivalent to the $T_{0}$-space $X$ of Theorem 14 , then the relation between $\Re, \subseteq$, and $X$ is said to be a map $m(\Re, \subseteq, X)$ of $\Re$ in $\subseteq$ defined by the family $X$. A map defined by a subfamily of $X$ is called a submap of $m(\Re, \mathfrak{S}, X)$. A map $m(\Re, \mathfrak{S}, X)$ is said to be a covering map or a map of $\Re$ on $\subseteq$ if $X$ covers $\subseteq$. A map $m(\Re, \subseteq, X)$ is said to be continuous if $\mathcal{X}$ is a continuous family. The index of the family $X$ is called the index of the map $m(\Re, \mathfrak{S}, X) . A$ map is said to be a Boolean map if the space $\mathfrak{S}$ is a bicompact Boolean space.

It is convenient to state explicitly the following elementary theorems.

THEOREM 15. If $\Re_{1}, \Im_{1}$ are topologically equivalent to $\Re_{2}, \Im_{2}$ respectively, then the existence of a map $m\left(\Re_{1}, \Im_{1}, X_{1}\right)$ implies and is implied by the existence of a map $m\left(\Re_{2}, \mathfrak{S}_{2}, X_{2}\right)$ whenever the equivalence between $\mathfrak{S}_{1}$ and $\mathfrak{S}_{2}$ carries the families $X_{1}$ and $X_{2}$ into one another.

THEOREM 16. If $\mathfrak{S}$ is a closed subset of a $T_{1}$-space $\mathfrak{T}$ and $X$ is a family of subsets of $\mathfrak{S}$, then the members of $\mathcal{X}$ are closed relative to $\subseteq$ if and only if they are closed relative to $\mathfrak{T}$; and the existence of a map $m(\Re, \subseteq, X)$ defined by the family $X$ in $\subseteq$ implies and is implied by the existence of a map $m(\Re, \mathfrak{T}, X)$ defined by the family $X$ in $\mathfrak{T}$. If $\mathfrak{S}$ is any subset of a $T_{1}$-space $\mathfrak{T}$ and $X$ is a family of subsets of $\mathfrak{S}$ closed relative to $\mathfrak{T}$, then the members of $\mathcal{X}$ are closed relative to $\mathfrak{S}$; and the existence of a map $m(\Re, \mathfrak{S}, X)$ implies and is implied by the existence of $a$ map $m(\Re, \mathfrak{T}, X)$. In particular, the existence of a map $m(\Re, \mathfrak{S}, X)$ implies and is implied by the existence of a map $m(\Re, \subseteq(X), X)$ or a map $m\left(\Re, S_{-}(X), X\right)$, where $X$ is a family of distinct non-void closed subsets of $\subseteq$.

In accordance with Theorem 15, we introduce the following definition.

Definition 8. If two maps $m\left(\Re_{1}, \mathfrak{S}_{1}, X_{1}\right)$ and $m\left(\Re_{2}, \mathfrak{S}_{2}, X_{2}\right)$ are related in the manner described in Theorem 15, they are said to be equivalent.

It is evident that equivalent maps are topologically indistinguishable. In particular, if one of two equivalent maps is continuous or covering, then the other is also; and two equivalent maps have the same index. For purposes of exposition, it is often convenient to recall that, by definition, a map $m(\Re, \subseteq, X)$ is equivalent to the "identical" map $m(X, \subseteq, X)$ in which the points of the space $X$ of Theorem 14 are represented by themselves as closed subsets of $\mathfrak{S}$.

We can now raise three general questions concerning maps: first, we in- 
quire what consequences flow from the imposition of various simple conditions upon $\Re, \mathfrak{S}$, or $X$ in the map $m(\Re, \mathfrak{S}, X)$; second, we inquire what reductions can be applied to a map $m(\Re, \subseteq, X)$ in order to obtain a simplified map $m\left(\Re, S^{*}, X^{*}\right)$; and, third, we inquire what connections link the theory of maps with the theory of topological images. We shall consider these three problems in succession.

In Theorem 14, we have already seen that in a map $m(\Re, \mathfrak{S}, X)$ or its equivalent $m(X, \subseteq, X)$ the space $\Re$ is a $T_{1}$-space if and only if the family $\mathcal{X}$ has a certain simple property. Beside this result we may now place the following theorem.

THEOREM 17. In a map $m(\Re, \mathfrak{S}, \mathcal{X})$, the condition that the members of $\mathcal{X}$ be disjoint sets is neither necessary nor sufficient for $\Re$ to be an $H$-space; but this condition becomes sufficient in the presence of either of the auxiliary conditions:

(1) $\subseteq$ is a normal space;

(2) $\mathfrak{S}$ is an $H$-space and the sets in $X$ are bicompact.

Let us first consider the sufficiency of the indicated condition when (1) or (2) is valid. If $\subseteq$ is normal and $\mathfrak{X}_{1}$ and $\mathfrak{X}_{2}$ are disjoint members of $\mathcal{X}$, the definition of normality establishes the existence of disjoint open sets $\mathscr{G}_{1}$ and $\mathfrak{B}_{2}$ in $\mathfrak{S}$ such that the closed sets $\mathfrak{X}_{1}, \mathfrak{X}_{2}$ satisfy the relations $\mathfrak{X}_{1} \subset \mathfrak{S}_{1}, \mathfrak{X}_{2} \subset \mathfrak{H}_{2}$. Hence any two such points in $X$ have disjoint neighborhoods in the neighborhood system of Theorem 14. It follows that our condition is sufficient for $X$ and $\Re$ to be $H$-spaces. Under condition (2), we construct analogous open sets $\mathscr{H}_{1}$ and $\mathscr{S}_{2}$ by the method used to show that every bicompact $H$-space is normal. If $\mathfrak{X}_{1}$ and $\mathfrak{X}_{2}$ are disjoint and bicompact in the $H$-space $\mathfrak{S}$, there exist disjoint open sets $B_{1}\left(B_{1}, B_{2}\right), B_{2}\left(B_{1}, B_{2}\right)$ containing respectively an arbitrary point $\mathbb{B}_{1}$ in $\mathfrak{X}_{1}$ and an arbitrary point $\dot{z}_{2}$ in $\mathfrak{X}_{2}$; for fixed $\mathbb{z}_{2}$ the sets $\mathfrak{G}_{1}\left(\mathbb{B}_{1}, \mathbb{z}_{2}\right)$ cover $\mathfrak{X}_{1}$ so that there exist points $B_{1}{ }^{(1)}, \cdots, B_{1}{ }^{(m)}$, dependent upon $\varepsilon_{2}$, for which $\mathbb{G}_{1}\left(\mathbb{B}_{2}\right)=\mathbb{B}_{1}\left(\mathbb{B}_{1}^{(1)}, \mathbb{B}_{2}\right) \cup \cdots \cup \mathfrak{S}_{1}\left(\mathbb{B}_{1}^{(m)}, \mathbb{B}_{2}\right) \supset \mathfrak{X}_{1}$; since the open sets $\mathfrak{B}_{2}\left(\mathbb{B}_{2}\right)=\mathfrak{G}_{2}\left(\mathbb{B}_{1}{ }^{(1)}, \mathbb{B}_{2}\right) \cdots \mathfrak{S}_{2}\left(\mathbb{B}_{1}{ }^{(m)}, \mathbb{B}_{2}\right)$ cover $\mathfrak{X}_{2}$, there exist points $\mathbb{B}_{2}^{(1)}, \cdots, \mathbb{B}_{2}{ }^{(n)}$ such that $\mathfrak{F}_{2}=\mathfrak{S}_{2}\left(\mathbb{B}_{2}{ }^{(1)}\right) \cup \cdots \cup \mathfrak{S}_{2}\left(\mathbb{B}_{2}{ }^{(n)}\right) \supset \mathfrak{X}_{2}$; since the open set $\mathfrak{S}_{1}\left(\mathbb{B}_{2}{ }^{(k)}\right)$ is disjoint from $\mathfrak{B}_{2}\left(\mathbb{Z}_{2}{ }^{(k)}\right)$ and contains $\mathfrak{X}_{1}$ for $k=1, \cdots, n$, the open sets $\mathfrak{S}_{1}=\mathfrak{G}_{1}\left(\mathfrak{B}_{2}^{(1)}\right) \ldots \mathfrak{B}_{1}\left(\mathbb{B}_{2}^{(n)}\right)$ and $\mathfrak{B}_{2}$ are disjoint and contain $\mathfrak{X}_{1}$ and $\mathfrak{X}_{2}$ respectively. Again it follows that our condition is sufficient for $\mathfrak{X}$ and $\Re$ to be $H$-spaces.

On the other hand, if $\subseteq$ is not normal, it is possible for two disjoint closed sets $\mathfrak{X}_{1}$ and $\mathfrak{X}_{2}$ to have the property that, whatever the open sets $\mathfrak{G}_{1}$ and $\mathfrak{G}_{2}$ containing $\mathfrak{X}_{1}$ and $\mathfrak{X}_{2}$ respectively, their intersection $\mathfrak{S}_{1} \mathfrak{H}_{2}$ is non-void. In such a situation we cannot conclude in general that the points $\mathfrak{X}_{1}$ and $\mathfrak{X}_{2}$ in $X$ have disjoint neighborhoods. Yet, if $\mathfrak{X}_{1}$ and $\mathfrak{X}_{2}$ have common points, they 
may still have disjoint neighborhoods, as can be seen from the following example: let $X$ consist of two closed sets $\mathfrak{X}_{1}$ and $\mathfrak{X}_{2}$ which have a common point but neither of which contains the other; then $X$ is an $H$-space.

The further examination of the effect of restrictions upon $\subseteq$ and $X$ is, in a sense, the chief aim of the following sections. Indeed, we shall see that the assumption that $\subseteq$ is a bicompact Boolean space has no effect upon the topological nature of the spaces $\Re$ which can be mapped in $\mathfrak{S}$. The relation of the properties of $X$ to those of $\Re$ is found to be rather complex. For the present, then, we leave the first problem with the remark that every bicompact Boolean space is normal and its closed subsets bicompact, thus rendering Theorem 17 applicable to such spaces.

We pass to our second problem. In Theorem 16 we have already noted that a map $m(\Re, \subseteq, X)$ can be modified, without the loss of essential information, through the suppression of $\mathfrak{S}^{\prime}(X)$ or of $\mathfrak{S}^{-1}(X)$. It will be observed that the second of these sets is an open set disjoint from all members of $X$ and that it is the maximal set with such properties. Now it is possible for an open set $\mathfrak{S}$ in $\subseteq$ to have points in common with some members of $X$ and yet contain no member of $X$. It is natural to examine the conditions under which such a set $\mathfrak{S}$ can be suppressed from a given map without disturbing the topological relations involved. We obtain the following result.

THEOREM 18. Let $\mathfrak{S}$ be an arbitrary open subset of a $T_{1}$-space $\mathfrak{S}$; let $\mathcal{X}$ be a family of distinct closed sets $\mathfrak{X}$ in $\mathfrak{S}$, none of which is contained in $\mathfrak{S}$; let $\mathfrak{T}$ be the set $\mathfrak{S}^{\prime}$ considered as a relative space; and let $\Upsilon$ be the family of the closed sets $\mathfrak{V}=\mathfrak{S}^{\prime} \mathfrak{X}$ in $\mathfrak{T}$. Under the topology introduced in Theorem 14, the spaces $X$ and $\Upsilon$ have the property that $\Upsilon$ is a continuous image of $X$ by virtue of the correspondence $\mathfrak{X} \rightarrow \mathfrak{Y}=\mathfrak{G}^{\prime} \mathfrak{X}$. In order that $X$ and $\Upsilon$ be topologically equivalent under this correspondence, it is necessary and sufficient that $\mathfrak{S}$ have the following property: if $\mathbb{S}$ is any open set in $\mathbb{S}$ and $\mathfrak{X}_{0}$ any member of $\mathcal{X}$ contained in $\mathbb{S}$, then there exists an open set $\mathfrak{S}_{0}$ such that $\mathfrak{S}_{0} \cup \mathfrak{S}$ contains $\mathfrak{X}_{0}$ and contains no member $\mathfrak{X}$ of $\mathcal{X}$ which is not contained in $\mathfrak{B S}$. In other words, this property of $\mathfrak{S}$ is characteristic for the possibility of suppressing $\mathfrak{S}$ from the map $m(\Re, \mathfrak{S}, X)$ so as to obtain a map $m(\Re, \mathfrak{T}, \Upsilon)$.

We consider the correspondence $\mathfrak{X} \rightarrow \mathfrak{V}=\mathfrak{S}^{\prime} \mathfrak{X}$. Since there is no $\mathfrak{X}$ for which $\mathfrak{S}^{\prime} \mathfrak{X}$ is void, this correspondence carries $X$ into $\Upsilon$ in a univocal manner. In order that the correspondence be biunivocal, it is necessary and sufficient that $\mathfrak{S}^{\prime} \mathfrak{X}_{1}=\mathfrak{S}^{\prime} \mathfrak{X}_{2}$ imply $\mathfrak{X}_{1}=\mathfrak{X}_{2}$ whenever $\mathfrak{X}_{1}$ and $\mathfrak{X}_{2}$ are members of $\mathfrak{X}$. In order to show that this correspondence is continuous, we consider an arbitrary neighborhood in $\Upsilon$ and its antecedent in $X$. Every open set in $\mathfrak{T}$ is obtained as a set $\mathfrak{S S}_{\mathfrak{S}^{\prime}}$ where $\mathbb{B}$ is an open set in $\mathfrak{S}$. Hence the neighborhoods in $\Upsilon$ are 
specified by the relations $\mathfrak{S}^{\prime} \mathfrak{X}=\mathfrak{Y} \subset \mathfrak{S}^{\prime}(\mathfrak{S}$, where $(\mathfrak{S}$ runs over the class of all open sets in $\mathfrak{S}$. Since each such relation is equivalent to the corresponding relation $\mathfrak{X} \subset \mathfrak{G} \cup \mathfrak{S}$, where $\mathfrak{G} \cup \mathfrak{S}$ is open in $\mathfrak{S}$, we see that each neighborhood in $\Upsilon$ has as its antecedent a neighborhood in $X$. This property of the correspondence identifies it as a continuous correspondence. In order that the correspondence be a topological equivalence it must be biunivocal and bicontinuous. When the condition of being biunivocal is met in accordance with the criterion noted above, we see therefore that the correspondence is an equivalence if and only if the inverse correspondence $\mathfrak{S}^{\prime} \mathfrak{X}=\mathfrak{Y} \rightarrow \mathfrak{X}$ is continuous. By the Cauchy criterion for continuity, ${ }^{*}$ the latter condition is satisfied if and only if $\mathbb{B} \supset \mathfrak{X}_{0}$, where $\mathfrak{B}$ is open in $\mathfrak{S}$, implies the existence of an open set $\mathfrak{S H}_{0} \mathfrak{S}^{\prime}$ in $\mathfrak{T}, \mathfrak{S}_{0}$ being open in $\mathfrak{S}$, such that $\mathfrak{V}_{0}=\mathfrak{S}^{\prime} \mathfrak{X}_{0}$ is contained in $\mathfrak{S}_{0} \mathfrak{S}^{\prime}$ while $\mathfrak{Y}=\mathfrak{S}^{\prime} \mathfrak{X} \subset \mathfrak{S}_{0} \mathfrak{S}^{\prime}$ implies $\mathfrak{X} \subset \mathfrak{G}$. This characteristic property is obviously equivalent to the one stated in the theorem. We complete the proof of the theorem by showing that the property stated in the theorem implies the property which was recognized above as sufficient to make the correspondence $\mathfrak{X} \rightarrow \mathfrak{Y}$ biunivocal. Suppose that $\mathfrak{X}_{1} \neq \mathfrak{X}_{2}$, the notation being chosen so that some point of $\mathfrak{X}_{2}$ does not belong to $\mathfrak{X}_{1}$. Then there exists an open set $\mathbb{B}$ in $\mathfrak{S}$ which contains $\mathfrak{X}_{1}$ but not $\mathfrak{X}_{2}$. By virtue of the property which is now being assumed for the set $\mathfrak{S}$, there exists an open set $\mathfrak{S}_{1}$ such that $\mathfrak{S}_{1} \cup \mathfrak{S}$ contains $\mathfrak{X}_{1}$ but contains no set $\mathfrak{X}$ which is not contained in $\mathfrak{B}$. In particular, then, $\mathfrak{G}_{1} \cup \mathfrak{S}$ does not contain $\mathfrak{X}_{2}$. It is therefore true that $\mathfrak{S}^{\prime} \mathfrak{X}_{1} \neq \mathfrak{S}^{\prime} \mathfrak{X}_{2}$ : for otherwise we should have $\mathfrak{S E}^{\prime} \mathfrak{X}_{2}=\mathfrak{S}^{\prime} \mathfrak{X}_{1} \subset \mathcal{S} \mathfrak{S E}^{\prime}$ and $\mathfrak{X}_{2} \subset \mathfrak{S}_{1} \cup \mathfrak{S}$, contrary to fact.

Because of the possibility of suppressing from a given map an open set such as that described in Theorem 18 , it is convenient to introduce the following definition.

Definition 9. A map $m(\Re, \subseteq, X)$ is said to be redundant if there exists a non-void open set $\mathfrak{S}$ which can be suppressed from $\subseteq$ in accordance with Theorem 18; the set $\mathfrak{S}$ is called a set of redundancy. A map in which no such set exists is said to be irredundant.

In general, a map will be redundant and will not even be convertible into an irredundant map by single or successive removals of sets of redundancy. We may, however, note two simple positive results in this connection. First, we have

THEOREM 19. If the map $m\left(\Re, \Im_{1}, X_{1}\right)$ is converted into a map $m\left(\Re, \Im_{2}, X_{2}\right)$ by the suppression of a set of redundancy $\mathfrak{S}_{1}$, and if the map $m\left(\Re, \mathfrak{S}_{2}, X_{2}\right)$ is converted in its turn into a map $m\left(\Re, \Im_{3}, X_{3}\right)$ by the suppression of a set of

* AH, pp. 53-54, Satz IV. 
redundancy $\mathfrak{S}_{2}$, then the set $\mathfrak{S}_{2}=\mathfrak{W}_{1} \cup \mathfrak{F}_{2}$ is a set of redundancy in the map $m\left(\Re, \subseteq_{1}, X_{1}\right)$ and its suppression yields the map $m\left(\Re, \subseteq_{3}, X_{3}\right)$.

Since $\Im_{2} \cdot$ is a closed proper subset of $\Im_{1}$ and $\Im_{3}$ is a closed proper subset of $\Im_{2}$, the set $\Im_{3}$ is a closed proper subset of $\Im_{1}$ and its complement $\mathfrak{S}_{3}^{\prime}=\mathfrak{S}_{2}^{\prime} \cup \mathfrak{S}_{2} \mathfrak{S}_{3}^{\prime}=\mathfrak{S}_{1} \cup \mathfrak{S}_{2}=\mathfrak{F}$ is a non-void open subset of $\mathfrak{S}_{1}$. The suppression of $\mathfrak{S}$ obviously converts $m\left(\Re, \mathfrak{S}_{1}, X_{1}\right)$ into $m\left(\Re, \mathfrak{S}_{3}, X_{3}\right)$. Hence $\mathfrak{S}$ is a set of redundancy in the first map.

Theorem 20. If $\mathcal{X}$ is densely distributed in $\subseteq$, the map $m(\Re, \subseteq, X)$ is irredundant.

The proof is obvious.

A further possibility of simplification in the theory of maps is that of replacing the neighborhood-system described in Theorem 14 by a suitable equivalent subsystem. In this connection we have the following result.

THEOREM 21. If the sets $\mathfrak{X}$ in $X$ are bicompact, then the subfamilies of $\mathcal{X}$ specified by the relations $\mathfrak{X} \subset \mathcal{H}$ constitute a basis in the $T_{0}$-space $X$ even when the set is is restricted to be a finite union of open sets belonging to an arbitrary fixed basis in 5 . The character of the $T_{0}$-space $X$ then does not exceed the character of $\mathfrak{S}$, if the latter is infinite.

We have to show that, if $\mathscr{S}$ is any open set in $\subseteq$ and $\mathfrak{X}$ any set in $X$ which is contained in $B$, then there exists a set $G_{1}$ of the indicated type for which

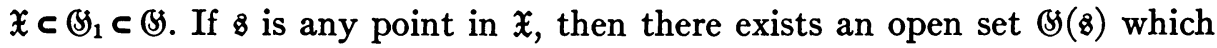
belongs to the chosen basis in $\subseteq$ and which has the property $\boldsymbol{B} \in \mathbb{S}(\mathbb{B}) \subset \mathbb{B}$. Since the sets $\mathfrak{B}(\mathfrak{B}), \mathbb{B} \in \mathfrak{X}$, cover the bicompact set $\mathfrak{X}$, there exist points $\mathbb{z}_{1}, \cdots, \mathbb{z}_{n}$ in $\mathfrak{X}$ such that $\mathfrak{X} \subset \mathbb{S}\left(\mathbb{B}_{1}\right) \cup \cdots \cup \mathfrak{S}\left(\mathbb{B}_{n}\right) \subset \mathbb{S}$. We may therefore put $\mathfrak{S}_{1}=\mathbb{S}\left(\mathbb{B}_{1}\right) \cup \ldots \cup \mathbb{S}\left(\mathbb{B}_{n}\right)$. Since the cardinal number of the class of open sets described in the theorem is equal to that of the chosen basis in $\mathfrak{S}$, when the latter is infinite, we see that the character of $X$ has the property asserted above.

We come finally to our third problem. Here we can give a complete solution, due originally to Kolmogoroff. ${ }^{*}$ It is embodied in the following theorem.

THEOREM 22. In order that the correspondence from $S(X)$ to $X$ given by $\mathbb{B} \rightarrow \mathfrak{X}$ where $\mathbb{z} \in \mathfrak{X}$ be univocal and continuous, it is necessary and sufficient that the family $X$ be continuous. Hence the map $m(\Re, \subseteq, X)$ characterizes $\Re$ as a continuous image of $\subseteq(X)$ if and only if the family $X$ is continuous.

A necessary and sufficient condition that the given correspondence be univocal is clearly that the sets belonging to $X$ be disjoint. When this condition is fulfilled, the continuity of the family $X$ is known to be both necessary

* AH, p. 61, footnote. 
and sufficient for the continuity of the correspondence from the space $\subseteq(X)$ to the $T_{0}$-space $\mathcal{X}$, by virtue of results due to Kolmogoroff. ${ }^{*}$ Hence we complete the proof of the present theorem by showing that in a continuous family the distinct members are disjoint. If $\mathfrak{X}_{1} \neq \mathfrak{X}_{2}$ we suppose that the notation is so chosen that $\mathfrak{X}_{2}$ contains a point $\&$ which does not belong to $\mathfrak{X}_{1}$. Then there exists an open set $\mathbb{B}$ in $\subseteq$ which contains $\mathfrak{X}_{1}$ but neither 8 nor $\mathfrak{X}_{2}$. By the continuity of the family $X$, there exists also an open set $\oiint_{1}$ which is contained in $\mathbb{B}$ and contains $\mathfrak{X}_{1}$ while every set $\mathfrak{X}$ such that $\mathfrak{X} \mathscr{S}_{1} \neq 0$ is contained in $\mathbb{B}$. Hence in particular we have $\mathfrak{X}_{2} \mathfrak{G}_{1}=0, \mathfrak{X}_{2} \subset \mathfrak{G}_{1}^{\prime}$. Since $\mathfrak{X}_{1} \subset \mathfrak{S}_{1}$, the sets $\mathfrak{X}_{1}$ and $\mathfrak{X}_{2}$ are disjoint.

With this, we have completed a general survey of the theory of maps. In concluding the present section, we shall summarize the preceding results as they apply to Boolean maps. We obtain the following theorem.

THeOREM 23. If $\mathcal{X}$ is an arbitrary family of distinct closed subsets $\mathfrak{X}$ of a bicompact Boolean space $\mathfrak{B}$, the topology introduced in $X$ by assigning each nonvoid subfamily of $X$ specified by a relation $\mathfrak{X} \subset \mathcal{B}$, where $\mathbb{B}$ is open in $\mathfrak{B}$, as a neighborhoud of every one of its elements is equivalent to that obtained by restricting the sets \&s to be bicompact as well as open. Since the closed set $\mathfrak{S}^{-}(\mathcal{X})$ is a bicompact Boolean space, it may be assumed without loss of generality that $\mathfrak{S}^{-}(\mathcal{X})=\mathfrak{B}$. Then the topological space $X$ has the properties:

(1) it is a $T_{0}$-space, of character not exceeding that of $\mathscr{B}$ if $\mathscr{B}$ is infinite;

(2) it is a $T_{1}$-space if and only if no member of the family $X$ contains another as a proper subset;

(3) it is an $H$-space if the distinct members of $X$ are disjoint. $A$ Boolean map $m(\Re, \mathfrak{B}, X)$ exists only if $\Re$ has the properties necessitated by (1), (2), and (3). The suppression of a set of redundancy from a Boolean map yields a Boolean map.

Since the bicompact open subsets constitute a basis in $\mathbb{B}$ in accordance with Theorems 1 and 2, the first statement of the present theorem is justified by reference to Theorem 21 . The second statement follows from Theorems 3 and 16. The properties (1), (2), and (3) are established by reference to Theorems 14,17 , and 21 . If $\mathfrak{S}$ is a set of redundancy in the map $m(\Re, \mathfrak{B}, X)$, then $\mathfrak{S}^{\prime}$ is a bicompact Boolean subspace of $\mathfrak{B}$ in accordance with Theorem 3; it follows that the suppression of $\mathfrak{S}$ yields a Boolean map.

2. Construction of Boolean maps. In this section, we propose to show that the bicompact Boolean spaces are universal mapping spaces; in other words, that every $T_{0}$-space can be mapped in an appropriate bicompact Boolean space. The constructive method which we employ is naturally

* Compare AH, p. 67, Satz VI. 
algebraic in character. Once the problem of finding some special type of universal mapping space has been proposed, the bicompact Boolean spaces appear to be peculiarly fitted to provide a solution. Thus the property of total-disconnectedness seems to be desirable if we are to avoid the consequences of the theorem which states that every continuous image of a connected space is connected;* and the property of bicompactness seems equally desirable if we are to recover from the theory of maps some of the known theorems concerning the representation of bicompact $H$-spaces. $\dagger$ Moreover, if we restrict attention for the moment to separable spaces, that is, to spaces of character not exceeding $\boldsymbol{\aleph}_{0}$, we have positive evidence in favor of our selection of Boolean spaces as universal mapping spaces. For it is known that every compact metric space or, equivalently, every bicompact separable $H$-space is a continuous image of the Cantor discontinuum $\mathfrak{D}$ and hence of the equivalent Boolean space $\mathfrak{B}_{\mathfrak{c}}, \mathfrak{c}=\boldsymbol{\aleph}_{0}$, of Theorems 9 and $13 . \ddagger$

In order to give a reasonably complete analysis of the theory of Boolean maps, we shall have to proceed in a somewhat more complicated way than would be necessary if we were concerned merely with solving the problem of universality raised in the preceding paragraph. We begin therefore with some propositions about topological algebra.

TheOREM 24 . Let $\Re$ be an arbitrary $T_{0}$-space, $B_{\Re}$ the Boolean ring of all subsets of $\Re, a_{\Re}$ the class of all nowhere dense subsets of $\Re$. Then $a_{\Re}$ is an ideal in $B_{\Re}$; and, if $A$ is any subring of $B_{\Re}$, the class a of all elements common to $a_{\Re}$ and $A$ is an ideal in $A$. The subclass $A_{\Re}$ of $B_{\Re}$ specified by any of the following equivalent conditions:

(1) $a^{-} a^{\prime} \epsilon \mathfrak{a}_{\Re}$; (2) $a a^{\prime}-\epsilon \mathfrak{a}_{\Re}$; (3) $a^{-} a^{\prime-}-\mathfrak{a}_{\Re}$;

(4) $a$ is congruent $\left(\bmod \mathfrak{a}_{\Re}\right)$ to an open subset of $\Re$;

(5) $a$ is congruent $\left(\bmod a_{\Re}\right)$ to a regular open subset of $\Re$;

(6) a is congruent $\left(\bmod a_{\Re}\right)$ to a closed subset of $\Re$;

(7) $a$ is congruent $\left(\bmod a_{\Re}\right)$ to its interior $a^{\prime-^{\prime}}$;

(8) $a$ is congruent $\left(\bmod a_{\Re}\right)$ to its closure $a^{-}$;

(9) $a$ is congruent $\left(\bmod a_{\Re}\right)$ to the regular open set $a^{-^{\prime-\prime}}$;

is a subring of $B_{\Re}$ containing the set $\Re$ as its unit and the class $a_{\Re}$ as an ideal. It is the subring of $B_{\Re}$ generated by $\mathfrak{a}_{\Re}$ and the class of all open (regular open, closed) subsets of $\Re$.

By definition the members of $\mathfrak{a}_{\Re}$ are characterized by the identity $a^{-\prime}=\Re$. To prove that $a_{\Re}$ is an ideal, we combine this characteristic property

* AH, p. 53, Corollary to Satz I.

$\dagger$ AH, pp. 96-98.

$\ddagger$ AH, pp. 85-88, 119-122. 
with $\mathrm{R}$ Theorem 16. Thus if $a$ and $b$ are nowhere dense, we have $\Re=a^{-1-}$ $=\left(a^{-\prime} \Re\right)^{-}=\left(a^{-\prime}\left(b^{-} \vee b^{-\prime}\right)\right)^{-}=\left(a^{-\prime} b^{-} \vee a^{-\prime} b^{-\prime}\right)^{-}=\left(a^{-\prime} b^{-}\right)^{-} \vee\left(a^{-\prime} b^{-\prime}\right)^{-}<a^{-\prime} b^{-}$

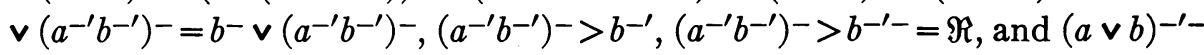
$=\left(a^{-} \vee b^{-}\right)^{\prime}=\left(a^{-\prime} b^{-\prime}\right)^{-}=\Re$, so that $a \vee b$ is also nowhere dense. Similarly, if $a$ is nowhere dense and $c<a$, then $a^{-}>c^{-}, c^{-\prime}>a^{-^{\prime}}$, and $c^{-\prime}>a^{--}=\Re$, so that $c$ is nowhere dense. The void set obviously belongs to $a_{\Re}$. It is thus.clear that $a_{\mathfrak{R}}$ is an ideal in $B_{\mathfrak{R}}$; and also that in any subring $A$ of $B_{\mathfrak{R}}$ the class $\mathfrak{a}$ of elements common to $\mathfrak{a}_{\Re}$ and $A$ is an ideal.

We shall next consider the class $A_{\Re}$ specified by the condition (3) above. First we observe that $A_{\Re}$ contains $a_{\Re}$ : for, if $a$ is nowhere dense, then $a^{-}$and $a^{-} a^{\prime}-<a^{-}$are nowhere dense. Then it is obvious from the symmetry of the condition (3) in $a$ and $a^{\prime}$ that $A_{\Re}$ contains $a$ if and only if it contains $a^{\prime}$. Furthermore, $A_{\Re}$ contains $a \vee b$ together with $a$ and $b$ : for the relations $(a \vee b)^{-}=a^{-} \vee b^{-}, \quad(a \vee b)^{\prime-}=\left(a^{\prime} b^{\prime}\right)^{-}<a^{\prime}-b^{\prime}-$ imply $(a \vee b)^{-}(a \vee b)^{\prime}-<a^{-} a^{\prime-}$ $\vee b^{-} b^{\prime}-\epsilon \mathfrak{a}_{\Re}$ when $a$ and $b$ are in $A_{\Re}$. Hence we see that $A_{\Re}$ is a subring of $B_{\Re}$, since it contains $a b=\left(a^{\prime} \vee b^{\prime}\right)^{\prime}$ and $a+b=a b^{\prime} \vee a^{\prime} b=\left(a^{\prime} \vee b\right)^{\prime} \vee\left(a \vee b^{\prime}\right)^{\prime}$ whenever it contains $a$ and $b$. The previous results show that $\mathfrak{a}_{\Re}$ is an ideal in $A_{\Re}$.

Now if $a$ is any open set in $\Re$, we have $\left(a^{-} a^{\prime-}\right)^{-{ }^{-}-}=\left(a^{-} a^{\prime}\right)^{-^{-}}=\left(a^{-} a^{\prime}\right)^{\prime-}$ $=\left(a^{-\prime} \vee a\right)^{-}=a^{-\prime} \vee \vee a^{-}>a^{-\prime} \vee a^{-}=\Re$, so that $a^{-} a^{\prime} \epsilon a_{\Re}, a \epsilon A_{\Re}$; and, if $a \equiv b\left(\bmod a_{\Re}\right)$ where $b$ is an open set in $\Re$, we have $a=(a+b)+b$ where $a+b \in \mathfrak{a}_{\mathfrak{R}} \subset A_{\mathfrak{R}}, b \in A_{\mathfrak{R}}$ and hence $a \epsilon A_{\mathfrak{R}}$. Thus (4) implies (3). Since the relation $a \equiv b\left(\bmod \mathfrak{a}_{\Re}\right)$ is equivalent to $a^{\prime} \equiv b^{\prime}\left(\bmod \mathfrak{a}_{\mathfrak{R}}\right)$, we see immediately that (6) also implies (3). It is trivial, from the preceding results, that (5) implies (3). Next we see by virtue of the relations $a^{-} a^{\prime}<a^{-} a^{\prime-}, a a^{\prime}-<a^{-} a^{\prime-}$ that (3) implies both (1) and (2). Now (1) obviously implies $a \equiv a^{-}\left(\bmod a_{\Re}\right)$ since $a^{-}+a=a^{-} a^{\prime} \vee a^{-\prime} a=a^{-} a^{\prime} \epsilon \mathfrak{a}_{\Re}$; and (2) similarly implies $a \equiv a^{\prime \prime \prime}\left(\bmod a_{\mathfrak{R}}\right)$. Thus (1) implies (8), (2) implies (7). It is evident that (8) implies (6) and that (7) implies (4). From the scheme of implications

$$
\text { (3) } \begin{aligned}
>(1) & \rightarrow(8) \rightarrow(6) \\
y(2) & \rightarrow(7) \rightarrow(4)
\end{aligned}
$$

we see that the conditions (1)-(4), (6)-(8) are equivalent. Since (3) implies $a \equiv a^{-}\left(\bmod a_{\Re}\right)$, since $a \equiv a^{-}\left(\bmod a_{\Re}\right)$ implies $a^{-} \in A_{\Re}$, and since (3) then implies $a^{-} \equiv a^{-^{\prime \prime}}\left(\bmod a_{\Re}\right)$, we conclude that (3) implies $(9) a \equiv a^{-^{\prime \prime}}\left(\bmod a_{\Re}\right)$. It is obvious that (9) implies (5). Thus by fitting the scheme of implications

$$
(3) \rightarrow(9) \rightarrow(5) \rightarrow(4)
$$

into the scheme given above, we see that the conditions (1)-(9) are equivalent. It is then obvious that $A_{\Re}$ is the subring of $B_{\Re}$ generated in the manner described above. 
We may remark that some of the conditions (1)-(9) may be cast into more geometrical form. Thus (1), (2), (3) assert respectively that the border, the frontier, or the boundary of $a$ is nowhere dense; (7) asserts that $a$ differs from its interior by a nowhere dense set; and (8) asserts that $a$ differs from its closure by a nowhere dense set.

While the regular open sets have little interest for us in the present chapter, they will later play an important rôle. Hence we shall enumerate some of their useful properties at this point.

THeOREM 25. Between the residual classes $\left(\bmod a_{\Re}\right)$ in the Boolean ring $A_{\Re}$ and the regular open sets in $\Re$, there exists a biunivocal correspondence such that each residual class contains the corresponding regular open set. The intersection of a finite number of regular open sets is a regular open set; but the union, in general, is not. If $a \equiv b\left(\bmod \mathfrak{a}_{\Re}\right)$ where $b$ is a regular open set, then the interior of $a$ is contained in $b$; and, if $a$ is an arbitrary subset of a regular open set $b$, then $a^{-1-}$ is a regular open set contained in $b$. The regular open sets in $\Re$ are characterized by the property that their borders coincide with the borders of their exteriors.

From the preceding theorem, we know that each residual class ( $\left.\bmod \mathfrak{a}_{\mathfrak{R}}\right)$ contains at least one regular open set. Thus we have to prove that, if $a$ and $b$ are both regular open sets, then $a \equiv b\left(\bmod \mathfrak{a}_{\Re}\right)$ implies $a=b$. Instead of establishing this result directly, we first show that, if $a$ and $b$ are both regular open sets, then their intersection is also such a set. We have $(a b)^{-}<a^{-} b^{-}$,

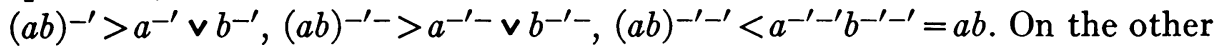
hand, if $c$ is an arbitrary set we have $c<c^{-}, c^{\prime}>c^{-^{\prime}}, c^{\prime-}>c^{-^{\prime}-}$, and $c^{\prime-^{\prime}}<c^{-^{\prime-}-^{\prime}}$. In particular, since $a b$ is open, we have $a b=(a b)^{\prime-^{\prime}}<(a b)^{-^{\prime-\prime}}$. We therefore conclude that $a b=(a b)^{-^{\prime-}{ }^{\prime}}$, as we wished to do. It is easy to see by examples that the union of regular open sets need not be a regular open set. We can now return to the previous question. To begin with, let us assume that the regular open sets $a$ and $b$ satisfy the relation $a>b$ in addition to the relation $a \equiv b\left(\bmod \mathfrak{a}_{\Re}\right)$. Then we see that $a b^{\prime}=a+b \in \mathfrak{a}_{\Re},\left(a b^{\prime}\right)^{-\prime}=\Re$. Hence we have $\left(a b^{\prime}\right)^{-}>a b^{\prime},\left(a b^{\prime}\right)^{-\prime}<a^{\prime} \vee b, \Re=\left(a b^{\prime}\right)^{-\prime}-<a^{\prime}-\vee b^{-}=a^{\prime} \vee b^{-}, b^{-}>a, b^{-1}<a^{\prime}$, $b^{-\prime}-<a^{\prime}-=a^{\prime}, b=b^{-^{\prime \prime}}>a$, and $a=b$. Passing now to the general case, we see that $a b$ is a regular open set satisfying the relations $a \equiv a a \equiv a b \equiv b b \equiv b$ $\left(\bmod a_{\Re}\right)$ and the relations $a>a b, b>a b$. Thus the result just obtained implies $a=a b=b$, as we wished to show. Now if $a \equiv b\left(\bmod \mathfrak{a}_{\Re}\right)$ where $b$ is a regular open set, we know from Theorem 24 that $a \equiv a^{\prime-^{\prime}} \equiv a^{-^{\prime-1}} \equiv b\left(\bmod a_{\Re}\right)$; and from the results proved above we know that $a^{\prime-^{\prime}}<a^{--^{\prime}}$ and that the regular open set $a^{\alpha^{\prime \prime}}$ coincides with $b$. Hence we see that the interior of $a$ is contained in $b$. We may state this result in the form: the modification of a regular 
open set by the addition $(\bmod 2)$ of a nowhere dense set may suppress interior points but cannot adjoin interior points. Next we consider the relations $a<b, b=b^{-^{\prime \prime}}$ : we then have $a^{-}<b^{-}, a^{-\prime}>b^{-^{\prime}}, a^{-^{\prime}}>b^{-^{\prime}}, a^{-^{\prime-\prime}}<b^{--^{\prime}}=b$, as stated above. Finally, we establish the characterization of the regular open sets given in the theorem. We have to prove that the relations $a=a^{\alpha^{\prime \prime}}$ and $a^{-} a^{\prime}=a^{\alpha^{-}} a^{-}$are equivalent. If $a=a^{-^{\prime \prime}}$, we have $a^{\prime}=a^{\alpha^{\prime}}$ and hence $a^{-} a^{\prime}=a^{-\prime}-a^{-}$as asserted. If $a^{-} a^{\prime}=a^{-\prime}-a^{-}$, we have $a^{\alpha^{\prime}} \vee a=a^{-^{\prime-}} \vee a^{-\prime}$, $a=a^{-}\left(a^{-\prime} \vee a\right)=a^{-}\left(a^{-^{\prime \prime}} \vee a^{-\prime}\right)=a^{-} a^{-!-^{\prime}}$, where $a^{-}>a^{-^{\prime-\prime}}$ by virtue of the relation $a^{-1}<a^{-\prime}$; and we therefore conclude that $a=a^{--^{\prime \prime}}$.

We now proceed to the algebraic construction of Boolean maps.

Theorem 26. Let $\Re$ be any non-void $T_{0}$-space, $A_{\Re}$ the Boolean ring of Theorem 24, and $A$ any subring of $A_{\Re}$ with the properties

(1) A contains the set $\Re$ as its unit;

(2) the interiors of sets in $A$ constitute a basis for $\Re$.

Then let $\mathfrak{B}=\mathbb{E}(A)$ be the representative bicompact Boolean space for the ring $A$; let $\mathrm{a}(\mathrm{r})$ be the class of all those sets $a$ in $A$ such that $a^{-}$does not contain the point $\mathfrak{r}$ in $\Re$; and let $\mathfrak{b}(\mathfrak{r})$ be the class of all those sets $b$ in $A$ such that $\mathfrak{r}$ is interior to $b$. The class $\mathfrak{a}(\mathfrak{r})$ is an ideal in $A$; and the closed set $\mathfrak{X}(\mathfrak{r})=\mathbb{F}^{\prime}(\mathfrak{a}(\mathfrak{r}))$ in $\mathfrak{E}(A)$ has the property

$$
\mathfrak{X}(\mathfrak{r})=\left(\sum_{a \in a(r)} \mathfrak{E}(a)\right)^{\prime}=\prod_{b \in \mathfrak{b}(\mathfrak{r})} \mathfrak{E}(b) .
$$

If $X$ is the family of all sets $\mathfrak{X}(\mathfrak{r})$ corresponding to points $\mathfrak{r}$ in $\Re$, the correspondence $\mathfrak{r} \rightarrow \mathfrak{X}(\mathfrak{r})$ defines a Boolean map $m(\Re, \mathfrak{B}, X)$. In order that a Boolean ring $A$ of subsets of $\Re$ have the properties demanded here, it is sufficient that it be the ring with unit $\Re$ generated by an arbitrary basis in $\Re$.

We first prove that $\mathfrak{a}(\mathfrak{r})$ is an ideal. Since $0^{-}=0$, the void set 0 is in $\mathfrak{a}(\mathfrak{r})$. If $a$ and $b$ are in $\mathfrak{a}(\mathfrak{r})$, the relation $(a \vee b)^{-}=a^{-} \vee b^{-}$shows that $a \vee b$ is also in $\mathfrak{a}(\mathfrak{r})$; and if $c<a$, where $a$ is in $\mathfrak{a}(\mathfrak{r})$, the relation $c^{-}<a^{-}$shows that $c$ is also in $\mathfrak{a}(\mathfrak{r})$. Thus $\mathfrak{a}(\mathfrak{r})$ is an ideal in accordance with $R$ Theorem 16 . Next we consider the relation between the ideal $\mathfrak{a}(\mathfrak{r})$ and the class $\mathfrak{b}(\mathfrak{r})$. We see at once that $a$ is in $\mathfrak{a}(\mathfrak{r})$ if and only if $b=a^{\prime}$ is in $\mathfrak{b}(\mathfrak{r})$ : for $a \epsilon \mathfrak{a}(\mathfrak{r})$ implies $\mathfrak{r} \epsilon a^{-\prime}=b^{\prime-^{\prime}}$ and $b \in \mathfrak{b}(\mathfrak{r})$ implies $\mathfrak{r} \epsilon b^{-^{\prime}}=a^{-^{\prime}}$. It is therefore evident that

$$
\mathfrak{X}(\mathfrak{r})=\mathbb{F}^{\prime}(\mathfrak{a}(\mathfrak{r}))=\left(\sum_{a \in a(\mathfrak{r})} \mathbb{E}(a)\right)^{\prime}=\prod_{a \in \mathfrak{a}(\mathfrak{r})} \mathbb{F}^{\prime}(a)=\prod_{a \in \mathfrak{a}(\mathfrak{r})} \mathbb{E}\left(a^{\prime}\right)=\prod_{\mathfrak{b} \in b(\mathfrak{r})} \mathfrak{E}(b)
$$

by virtue of $\mathrm{R}$ Theorem 67 . It is therefore easy to determine the significance of the relation $\mathfrak{X}(\mathfrak{r}) \subset \mathfrak{E}(b)$ when $b$ is an element of $A$. It is equivalent successively to the relations $\mathbb{F}^{\prime}(\mathfrak{a}(\mathfrak{r})) \subset \mathbb{E}(b), \mathbb{E}(\mathfrak{a}(\mathfrak{r})) \supset \mathbb{E}\left(b^{\prime}\right)=\mathbb{F}^{\prime}(b), b^{\prime} \in \mathfrak{a}(\mathfrak{r})$, and 
$b \in \mathfrak{b}(\mathfrak{r})$. Thus the relation $\mathfrak{X}(\mathfrak{r}) \subset \mathfrak{E}(b)$ holds if and only if $\mathfrak{r}$ is interior to the set $b$. From this fact we can deduce that the sets $\mathfrak{X}(\mathfrak{r})$ and $\mathfrak{X}(\mathfrak{B})$ corresponding to distinct points $\mathfrak{r}$ and $\&$ in $\Re$ are necessarily distinct sets. Since the interiors of sets in $A$ constitute a basis in $\Re$, there exists a set $b$ in $A$ to which just one of the two points $\mathfrak{r}$ and $z$ is interior. If we suppose that the notation is chosen so that $\mathfrak{r}$ is interior to $b$ while $\mathbb{B}$ is not, we conclude that $\mathbb{E}(b)$ contains $\mathfrak{X}(\mathfrak{r})$ but not $\mathfrak{X}(\mathfrak{z})$. Hence we have $\mathfrak{X}(\mathfrak{r}) \neq \mathfrak{X}(\mathfrak{z})$ whenever $\mathfrak{r} \neq \mathfrak{B}$.

We shall now interpret the foregoing algebraic facts topologically. In the bicompact Boolean space $\mathfrak{B}=\mathfrak{E}(A)$, defined as in Theorem 1, the sets $\mathbb{E}(a)$, $a \epsilon A$, are bicompact open sets constituting a basis; and the sets $\mathfrak{X}(\mathfrak{r})$ are closed subsets of $\mathscr{B}$ in accordance with Theorems 1 and 4 . Since the closed sets $\mathfrak{X}(\mathfrak{r}), \mathfrak{r} \in \Re$ are distinct and obviously non-void, they constitute a family $X$ to which Theorems 14 and 23 can be applied. The subfamilies of $X$ specified by the relations $\mathfrak{X}(\mathfrak{r}) \subset \mathbb{E}(b), b \in A$, then constitute a basis in the $T_{0}$-space $\mathcal{X}$, as asserted in Theorem 23. The correspondence $\mathfrak{r} \rightarrow \mathfrak{X}(\mathfrak{r})$ carries $\Re$ into $X$ in a biunivocal way. Since the sets $b^{\prime \prime}$, where $b$ is in $A$, constitute a basis in $\Re$ and since the relations $r \in b^{\prime-\prime}$ and $\mathfrak{X}(\mathfrak{r}) \subset \mathbb{E}(b)$ are equivalent, we now see that the correspondence sets up a topological equivalence between the spaces $X$ and $\Re$. By Definition 7 , this relation between $\Re, \mathfrak{B}$, and $X$ is a Boolean map $m(\Re, \mathfrak{B}, X)$. The closing remark of the theorem is an obvious consequence of Theorem 24 ; it shows that the existence of a ring $A$ of the required type offers no difficulty.

For convenience of reference to this fundamental result we introduce the following terminology.

Definition 10. If $\Re$ is a $T_{0}$-space and $A_{\Re}$ the Boolean ring described in Theorem 24, then any subring $A$ of $A_{\Re}$ with the properties (1) and (2) of Theorem 26 is called a basic ring of $\Re$; the ring $A_{\Re}$ is called the complete basic ring of $\Re$.

Definition 11. If $\Re$ is a $T_{0}$-space and $A$ is a basic ring of $\Re$, then the Boolean map $m(\Re, \mathfrak{B}, X)$ defined by $A$ in the manner described in Theorem 26 is called an algebraic (Boolean) map of $\Re$; and the map defined by the ring $A_{\Re}$ is called the complete algebraic (Boolean) map of $\Re$.

Before proceeding to a more detailed analysis of the algebraic maps, we pause to consider the general significance of Theorems 23 and 26. It is clear that we can summarize the situation in the following terms.

THEOREM 27. The algebraic theory of Boolean rings (with unit) is mathematically equivalent to the topological theory of $T_{0}$-spaces.

In the first place, Theorem 23 shows that any family $x$ of distinct non- 
void closed sets $\mathfrak{X}$ in a bicompact Boolean space $\mathfrak{B}$ may be regarded, under a suitable topology, as a $T_{0}$-space; but, since the open sets $\mathfrak{X}^{\prime}$ in $\mathscr{B}$ correspond by Theorems 1 and 4 to ideals in a Boolean ring with unit which has $\mathscr{B}$ as its representative, we actually have a representation of certain ideals and their algebraic relations by means of the indicated $T_{0}$-space and its topological properties. On the other hand Theorem 26 shows that such representative $T_{0}$-spaces are entirely arbitrary and unrestricted. Thus the algebraic structure of families of ideals in a Boolean ring with unit is exactly reflected in the topological structure of $T_{0}$-spaces. It follows that the complete analysis of the ideal structure of Boolean rings is as complicated as the analysis of the structure of all $T_{0}$-spaces. In the second place, Theorems 23 and 26 may be viewed, from another angle, as placing the study of all $T_{0}$-spaces on a purely combinatorial basis: for any such space is completely described as a configuration of ideals in a Boolean ring; and, as we have remarked elsewhere, the postulates for Boolean rings are postulates for an abstract algebra of combinations. These theorems, furthermore, reduce the construction of $T_{0}$-spaces to a kind of tactical game with the closed subsets of bicompact Boolean spaces.* We may remark that the requirement that the Boolean rings in the foregoing discussion should have units, does not affect the range of our comments in a serious way: for the adjunction of a unit to a Boolean ring is an essentially trivial operation, as we have already seen in $\mathrm{R}$ Theorem 1 and Theorem 8.

We now return to the further discussion of algebraic Boolean maps.

THEOREM 28. If $\mathfrak{a}$ is the ideal of all nowhere dense sets in a basic ring $A$ of a $T_{0}$-space $\Re$, then the algebraic map $m(\Re, \mathfrak{B}, \mathcal{X})$ defined by $A$ has the following properties:

(1) in order that $\mathfrak{E}(a), a \epsilon A$, contain no set $\mathfrak{X}(\mathfrak{r})$ in $\mathcal{X}$, it is necessary. and sufficient that $a \in \mathfrak{a}$;

(2) the set $\mathfrak{S}=\mathbb{E}(\mathfrak{a})$ is the maximal open subset of $\mathfrak{B}$ which contains no set $\mathfrak{X}(\mathfrak{r})$ in $X$

(3) the points of $\Re$ specified by the relations $\mathfrak{X}(\mathfrak{r}) \subset \mathfrak{E}(a), \mathfrak{X}(\mathfrak{r}) \mathfrak{F}(a) \neq 0$, where $a$ is in $A$, constitute the open set $a^{\prime-\prime}$ and the closed set $a^{-}$respectively. If $\Re$ is an $H$-space, then $\mathfrak{r} \neq \mathbb{B}$ implies $\mathfrak{X}(\mathfrak{r}) \mathfrak{X}(\mathbb{z}) \mathfrak{E}^{\prime}(\mathfrak{a})=0$; and, if in addition the basic ring $A$ actually contains a basis for $\Re$, then $\mathfrak{r} \neq \mathbb{B}$ implies $\mathfrak{X}(\mathfrak{r}) \mathfrak{X}(\mathfrak{B})=0$. The sufficient condition of Theorem 23 thus becomes necessary in the latter case.

* The tactical method has been extensively used, for example, by Alexandroff and Urÿsohn, Mémoire sur les espaces topologiques compacts, Verhandelingen der Koninklijke Akademie van Wetenschappen te Amsterdam, Eerste Sectie, Deel XIV, No. 1 (1929). Their constructions are usually carried out in the Euclidean plane, however. 
In particular, in order that $\Re$ be an $H$-space, it is necessary and sufficient that in its complete map $m(\Re, \mathfrak{B}, X)$ the sets in $X$ be disjoint.

In the proof of Theorem 26 we have already established the first part of (3): the relations $\mathfrak{r} \epsilon a^{\prime{ }^{\prime}}$ and $\mathfrak{X}(\mathfrak{r}) \subset \mathfrak{E}(a)$ are equivalent. The second part can be proved from the first as follows: the relation $\mathfrak{X}(\mathfrak{r}) \mathfrak{E}(a) \neq 0$ holds if and only if $\mathfrak{X}(\mathfrak{r})$ is not contained in $\mathbb{E}\left(a^{\prime}\right)=\mathfrak{E}^{\prime}(a)$ and is therefore equivalent to the relation $\mathfrak{r} \epsilon\left(\left(a^{\prime}\right)^{\prime-{ }^{\prime}}\right)^{\prime}=a^{-}$. It is now clear that (3) implies (1): for, in order that the set $\mathbb{E}(a)$ contain no set $\mathfrak{X}(\mathfrak{r})$ it is necessary and sufficient that $a^{\prime-^{\prime}}=0$; but Theorem 24 permits us to use the relations $a \equiv a^{\prime-^{\prime}}\left(\bmod a_{\mathfrak{R}}\right), a^{\prime-^{\prime}}<a^{-^{\prime \prime}}$ to show that $a^{\prime-\prime}=0$ implies $a \equiv 0\left(\bmod a_{\Re}\right)$ or $a \in \mathfrak{a}$ and that $a \in \mathfrak{a}$ implies $a^{\prime-^{\prime}}<\left(a^{-\prime}-\right)^{\prime}=\Re^{\prime}=0$. We now show that (1) implies (2). If $\mathfrak{X}(\mathfrak{r})$ is contained in $\mathfrak{E}(\mathfrak{a})$, the open sets $\mathbb{E}(a)$, aєa , cover $\mathfrak{X}(\mathfrak{r})$; since $\mathfrak{X}(\mathfrak{r})$ is bicompact, there exist elements $a_{1}, \cdots, a_{n}$ such that $\mathbb{E}(a)=\mathbb{E}\left(a_{1}\right) \cup \cdots \cup \quad \mathbb{E}\left(a_{n}\right) \supset \mathfrak{X}(\mathfrak{r})$, where $a_{1}, \cdots, a_{n}$ and $a=a_{1} \vee \cdots \vee a_{n}$ belong to the ideal $\mathfrak{a}$; and consequently we have a contradiction. Thus $\mathfrak{E}(\mathfrak{a})$ contains no set $\mathfrak{X}(\mathfrak{r})$. Since every open set in $\mathfrak{B}=\mathbb{E}(A)$ is a union of sets $\mathbb{E}(a), a \epsilon A$, an open set which contains no set $\mathfrak{X}(\mathfrak{r})$ must be a union of sets $\mathbb{E}(a), a \in \mathfrak{a}$, and must therefore be contained in $\mathbb{E}(\mathfrak{a})$. Hence $\mathfrak{E}(\mathfrak{a})$ is the maximal open set which contains no set $\mathfrak{X}(\mathfrak{r})$ in $X$.

We shall now prove that, if $\Re$ is an $H$-space, the relation $\mathfrak{r} \neq \mathbb{8}$ implies $\mathfrak{X}(\mathfrak{r}) \mathfrak{X}(\mathfrak{g}) \mathbb{F}^{\prime}(\mathfrak{a})=0$. By hypothesis, the basic ring $A$ contains elements $a$ and $b$

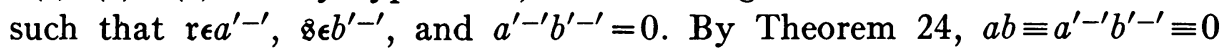
$\left(\bmod \mathfrak{a}_{\mathfrak{R}}\right)$ so that $a b \epsilon \mathfrak{a}$. Hence we have $\mathfrak{X}(\mathfrak{r}) \mathfrak{X}(\mathfrak{B}) \subset \mathbb{E}(a) \mathfrak{E}(b)=\mathbb{E}(a b) \subset \mathbb{E}(\mathfrak{a})$ by (3); and we conclude that $\mathfrak{X}(\mathfrak{r}) \mathfrak{X}(\mathfrak{B}) \mathfrak{F}^{\prime}(\mathfrak{a})=0$. In case $A$ contains a basis for $\Re$, we can select elements $a$ and $b$ which are open sets in $\Re$ and which have the properties $\mathfrak{r} \epsilon a=a^{\prime \prime \prime}, 8 \in b=b^{\prime-\prime}, a b=0$. We find that $\mathfrak{X}(\mathfrak{r}) \mathfrak{X}(\mathfrak{z})=0$. The proof of the theorem is thereby completed.

It is of considerable interest to compare the different algebraic maps of a given $T_{0}$-space $\Re$ and to examine the effect of suppressing the set $\mathfrak{S}=\mathbb{E}(\mathfrak{a})$ from a given algebraic map of $\Re$. We obtain the following result.

THEOREM 29. Let $m\left(\Re, \mathfrak{F}\left(A_{\Re}\right), X\right)$ be the complete algebraic map of $a$ $T_{0}$-space $\Re$; let $m\left(\Re, \&(A), X^{A}\right)$ be an arbitrary algebraic map of $\Re$ defined by a basic ring $A$; let $\mathfrak{a}_{\Re}$ and $a$ be the ideals of nowhere dense sets in $A_{\Re}$ and in $A$ respectively; and let $\mathfrak{p}=f(\mathfrak{q})$ be the function defining the bicontinuous univocal correspondence of $\&(A)$ with $\&\left(A_{\Re}\right)$ in accordance with the fact that $A$ is a sub'ring of $A_{\Re}$ with the same unit $\Re$. Then the indicated correspondence has the properties:

(1) $f^{-1}(\mathfrak{F}(\mathfrak{a})) \subset \mathbb{E}\left(\mathfrak{a}_{\mathfrak{R}}\right), f^{-1}\left(\mathfrak{F}^{\prime}(\mathfrak{a})\right) \supset \mathbb{F}^{\prime}\left(\mathfrak{a}_{\mathfrak{R}}\right), f\left(\mathfrak{F}^{\prime}\left(\mathfrak{a}_{\mathfrak{R}}\right)\right)=\mathbb{F}^{\prime}(\mathfrak{a})$;

(2) if $\mathfrak{Z}^{A}(\mathfrak{r})=f^{-1}\left(\mathfrak{X}^{A}(\mathfrak{r})\right)$, then $\mathfrak{Z}^{A}(\mathfrak{r}) \supset \mathfrak{X}(\mathfrak{r}), f(\mathfrak{X}(\mathfrak{r}))=f\left(\mathfrak{Z}^{A}(\mathfrak{r})\right)=\mathfrak{X}^{A}(\mathfrak{r})$;

(3) if $\mathfrak{Y}(\mathfrak{r})=\mathfrak{X}(\mathfrak{r}) \mathfrak{E}^{\prime}\left(\mathfrak{a}_{\mathfrak{R}}\right)$ and $\mathfrak{Y}^{A}(\mathfrak{r})=\mathfrak{X}^{A}(\mathfrak{r}) \mathfrak{E}^{\prime}(\mathfrak{a})$, then $f^{-1}\left(\mathfrak{Y}^{A}(\mathfrak{r})\right) \mathbb{E}^{\prime}\left(\mathfrak{a}_{\mathfrak{R}}\right)$ $=\mathfrak{Z}^{A}(\mathfrak{r}) \mathfrak{E}^{\prime}\left(\mathfrak{a}_{\mathfrak{R}}\right)=\mathfrak{X}(\mathfrak{r}) \mathfrak{F}^{\prime}\left(\mathfrak{a}_{\mathfrak{R}}\right)=\mathfrak{Y}(\mathfrak{r}), f(\mathfrak{Y}(\mathfrak{r}))=\mathfrak{Y}^{A}(\mathfrak{r})$. 
The sets $\mathfrak{Y}^{A}(\mathfrak{r})$ constitute a family $\Upsilon^{\boldsymbol{A}}$ of non-void closed subsets densely distributed in the bicompact Boolean space $\mathbb{F}^{\prime}(\mathfrak{a})$. Under the topology of Theorem 14, the $T_{0}$-spaces $\Upsilon^{A}$ corresponding to different basic rings $A$ are all topologically equivalent; in particular the space $\Upsilon$ corresponding to the ring $A=A_{\Re}$ and the space $\Upsilon^{A}$ corresponding to an arbitrary basic ring $A$ are topologically equivalent by virtue of the correspondence $\mathfrak{Y}(\mathfrak{r}) \longleftrightarrow \mathfrak{Y}^{A}(\mathfrak{r})$. If $\Re^{*}$ is any $T_{0 \text {-space topologi- }}$ cally equivalent to the spaces $\Upsilon^{A}$, then $\Re^{*}$ is a continuous image of $\Re$. When $\Re$ has infinite character, the character of $\Re^{*}$ does not exceed that of $\Re$. When $\Re$ is an $H$-space, $\Re^{*}$ is also an $H$-space; and $\Re^{*}$ is a biunivocal continuous image of $\Re$. The suppression of the open set $\mathfrak{E}(\mathfrak{a})$ from the algebraic map $m\left(\Re, \mathfrak{F}(A), X^{A}\right)$ yields an irredundant Boolean map $m\left(\Re^{*}, \mathbb{E}^{\prime}(\mathfrak{a}), \Upsilon^{A}\right)$.

Since $A$ is a subring of $A_{\Re}$ by hypothesis, Theorem 7 establishes the existence of a continuous univocal correspondence from $\&\left(A_{\Re}\right)$ to $\&(A)$. Since $\mathbb{E}\left(A_{\Re}\right)$ is bicompact, the correspondence is necessarily bicontinuous. $\dagger$ The correspondence was so defined that, if the element $a$ in $A$ be considered as an element $a_{\Re}=a$ of $A_{\Re}$, then $f^{-1}(\mathbb{E}(a))=\mathbb{E}\left(a_{\Re}\right)$. Since $a \in \mathfrak{a}$ if and only if $a=a_{\Re} \in \mathfrak{a}_{\Re}$ and $a=a_{\Re} \epsilon A$, we have

$$
f^{-1}(\mathfrak{E}(\mathfrak{a}))=f^{-1}\left(\sum_{a \in \mathfrak{a}} \mathfrak{E}(a)\right)=\sum_{a \in \mathfrak{a}} f^{-1}(\mathfrak{F}(a))=\sum_{a_{\mathfrak{R}} \in \mathbf{a}_{\mathfrak{R}}} \mathbb{E}\left(a_{\mathfrak{R}}\right) \subset \mathbb{F}\left(\mathfrak{a}_{\mathfrak{R}}\right),
$$

and hence also $f^{-1}\left(\mathfrak{F}^{\prime}(\mathfrak{a})\right) \supset \mathfrak{F}^{\prime}\left(\mathfrak{a}_{\Re}\right)$. The sets $f^{-1}(\mathfrak{F}(\mathfrak{a})), f^{-1}\left(\mathfrak{F}^{\prime}(\mathfrak{a})\right)$ are respectively open and closed because of the continuity of $f$. If we now make use of the bicontinuity of $f$, we see that $f\left(\mathbb{E}^{\prime}\left(\mathfrak{a}_{\Re}\right)\right)$ is a closed set contained in $\mathbb{E}^{\prime}(\mathfrak{a})$. If it does not coincide with $\mathbb{E}^{\prime}(\mathfrak{a})$, there exists an element $a$ which belongs to $A$ but not to $\mathfrak{a}$ and which has the property that $\mathbb{E}(a) f\left(\mathbb{E}^{\prime}\left(\mathfrak{a}_{\mathfrak{R}}\right)\right)=0$. By Theorem 28 , the relation $\mathbb{E}(a) \mathbb{E}^{\prime}(\mathfrak{a}) \neq 0$ implies the existence of a point $\mathfrak{r}$ in $\Re$ such that $\mathfrak{X}^{A}(\mathfrak{r}) \subset \mathbb{E}(a)$ or, equivalently, $\mathfrak{r} \in a^{\prime{ }^{\prime}}$. Interpreting the latter relation in terms of the map $m\left(\Re, \&\left(A_{\Re}\right), X\right)$ we see that the element $a=a_{\Re}$ in $A_{\Re}$ has the property $\mathbb{E}\left(a_{\Re}\right) \supset \mathfrak{X}(\mathfrak{r})$. Hence the set $f^{-1}(\mathbb{E}(a))=\mathbb{E}\left(a_{\Re}\right)$ has a point in common with $\mathscr{E}^{\prime}\left(\mathfrak{a}_{\Re}\right)$ in accordance with Theorem 28. Since $f\left(\mathbb{E}\left(a_{\Re}\right) \mathfrak{E}^{\prime}\left(\mathfrak{a}_{\mathfrak{R}}\right)\right) \subset f\left(\mathbb{E}\left(a_{\mathfrak{R}}\right)\right) f\left(\mathbb{E}^{\prime}\left(\mathfrak{a}_{\mathfrak{R}}\right)\right)=\mathbb{E}(a) f\left(\mathbb{E}^{\prime}\left(\mathfrak{a}_{\mathfrak{R}}\right)\right)$, we reach the contradiction that $\mathbb{E}(a) f\left(\mathbb{E}^{\prime}\left(\mathfrak{a}_{\Re}\right)\right) \neq 0$. Hence we must have $f\left(\mathbb{E}^{\prime}\left(\mathfrak{a}_{\Re}\right)\right)=\mathbb{E}^{\prime}(\mathfrak{a})$. This completes the proof of (1) above. Since the relations $\mathfrak{r} \in b^{\prime-\prime}, \mathfrak{X}^{A}(\mathfrak{r}) \subset \mathbb{E}(b)$, $\mathfrak{X}(\mathfrak{r}) \subset \mathbb{E}\left(b_{\Re}\right)$ for $b_{\Re}=b \epsilon A$ are equivalent, we have

$$
\mathfrak{Z}^{A}(\mathfrak{r})=f^{-1}\left(\mathfrak{X}^{A}(\mathfrak{r})\right)=f^{-1}\left(\prod_{b \in \mathfrak{b}(\mathfrak{r}),}(\mathfrak{s}(b))=\prod_{b \in \mathfrak{b}(\mathfrak{r})} f^{-1}(\mathfrak{E}(\mathfrak{b}))=\prod_{b_{\mathfrak{R}} \in \mathfrak{b}(\mathfrak{r})}\left(\mathfrak{E}\left(b_{\Re}\right) \supset \mathfrak{X}(\mathfrak{r})\right.\right.
$$

$\dagger$ AH, p. 95, Satz II. 
by virtue of Theorem 26. If we make use of the bicontinuity of the correspondence $f$, we see that $f(\mathfrak{X}(\mathfrak{r}))$ is a closed subset of $\mathfrak{X}^{A}(\mathfrak{r})$. If it does not coincide with $\mathfrak{X}^{A}(\mathfrak{r})$, then there exists a set $b$ in $A$ such that $\mathbb{E}(b)$ contains $f(\mathfrak{X}(\mathfrak{r}))$ but not $\mathfrak{X}^{A}(\mathfrak{r})$. If we put $b=b_{\Re} \epsilon A$, we see that $\mathfrak{E}\left(b_{\Re}\right)=f^{-1}(\mathfrak{E}(b)) \supset \mathfrak{X}(\mathfrak{r})$ and conclude that $r \in b_{\Re^{\prime}}{ }^{\prime}=b^{\prime-\prime}$. On the other hand, the fact that $\&(b)$ does not contain $\mathfrak{X}^{A}(\mathfrak{r})$ leads to the contradiction that $\mathfrak{r}$ does not belong to $b^{\prime{ }^{\prime}}$. We therefore conclude that $f(\mathfrak{X}(\mathfrak{r}))=\mathfrak{X}^{A}(\mathfrak{r})$. By definition we have $f\left(\mathfrak{Z}^{A}(\mathfrak{r})\right)=\mathfrak{X}^{A}(\mathfrak{r})$. This completes the proof of (2) above. From the previous results, we know that

$$
\begin{aligned}
& f^{-1}\left(\mathfrak{Y}^{A}(\mathfrak{r})\right) \mathfrak{F}^{\prime}\left(\mathfrak{a}_{\mathfrak{R}}\right)=f^{-1}\left(\mathfrak{X}^{A}(\mathfrak{r}) \mathfrak{F}^{\prime}(\mathfrak{a})\right) \mathfrak{F}^{\prime}\left(\mathfrak{a}_{\mathfrak{R}}\right)=f^{-1}\left(\mathfrak{X}^{A}(\mathfrak{r})\right) f^{-1}\left(\mathfrak{F}^{\prime}(\mathfrak{a})\right) \mathfrak{F}^{\prime}\left(\mathfrak{a}_{\mathfrak{R}}\right) \\
& =\mathfrak{Z}^{A}(\mathfrak{r}) \mathfrak{F}^{\prime}\left(\mathfrak{a}_{\mathfrak{R}}\right) \supset \mathfrak{X}(\mathfrak{r}) \mathfrak{F}^{\prime}\left(\mathfrak{a}_{\mathfrak{R}}\right)=\mathfrak{Y}(\mathfrak{r}), \\
& f(\mathfrak{Y}(\mathfrak{r}))=f\left(\mathfrak{X}(\mathfrak{r}) \mathfrak{F}^{\prime}\left(\mathfrak{a}_{\mathfrak{R}}\right)\right) \subset f(\mathfrak{X}(\mathfrak{r})) f\left(\mathfrak{E}^{\prime}\left(\mathfrak{a}_{\mathfrak{R}}\right)\right)=\mathfrak{X}^{A}(\mathfrak{r}) \mathfrak{E}^{\prime}(\mathfrak{a})=\mathfrak{Y}^{A}(\mathfrak{r}), \\
& f\left(f^{-1}\left(\mathfrak{Y}^{A}(\mathfrak{r})\right) \mathfrak{F}^{\prime}\left(\mathfrak{a}_{\mathfrak{R}}\right)\right)=\mathfrak{Y}^{A}(\mathfrak{r}) .
\end{aligned}
$$

If we can show that $\mathfrak{Z}^{A}(\mathfrak{r}) \mathfrak{E}^{\prime}\left(\mathfrak{a}_{\mathfrak{R}}\right)=\mathfrak{X}(\mathfrak{r}) \mathfrak{E}^{\prime}\left(\mathfrak{a}_{\mathfrak{R}}\right)$, we can then conclude that $\mathfrak{Y}(\mathfrak{r})=f^{-1}\left(\mathfrak{Y}^{A}(\mathfrak{r})\right) \mathfrak{F}^{\prime}\left(\mathfrak{a}_{\mathfrak{R}}\right), f(\mathfrak{Y}(\mathfrak{r}))=\mathfrak{Y}^{A}(\mathfrak{r})$, thus completing the proof of (3). Since $\mathfrak{X}(\mathfrak{r}) \mathbb{E}^{\prime}\left(\mathfrak{a}_{\Re}\right)$ is a closed subset of $\mathfrak{Z}^{A}(\mathfrak{r}) \mathbb{E}^{\prime}\left(\mathfrak{a}_{\mathfrak{R}}\right)$, the assumption that it is a proper subset permits us to find an element $a_{\Re}$ in $A_{\Re}$ such that $\&\left(a_{\Re}\right)$ contains $\mathfrak{X}(\mathfrak{r}) \mathscr{E}^{\prime}\left(\mathfrak{a}_{\mathfrak{R}}\right)$ but not $\mathfrak{Z}^{A}(\mathfrak{r}) \mathfrak{E}^{\prime}\left(\mathfrak{a}_{\mathfrak{R}}\right)$. The closed set $\mathfrak{X}(\mathfrak{r}) \mathbb{E}^{\prime}\left(a_{\Re}\right)$ is then contained in $\mathbb{E}\left(a_{\Re}\right)$. Hence there exists an element $b_{\Re}$ in $a_{\Re}$ such that $\mathbb{E}\left(b_{\Re}\right)$ contains $\mathfrak{X}(\mathfrak{r}) \mathfrak{F}^{\prime}\left(a_{\Re}\right)$. The element $a_{\Re} \vee b_{\Re}$ then has the property that $\mathfrak{E}\left(a_{\Re} \vee b_{\Re}\right)$ $=\mathbb{E}\left(a_{\Re}\right) \vee \mathfrak{E}\left(b_{\mathfrak{R}}\right)$ contains $\mathfrak{X}(\mathfrak{r})$ and $\mathfrak{X}(\mathfrak{r}) \mathfrak{E}^{\prime}\left(\mathfrak{a}_{\mathfrak{R}}\right)$ but not $\mathfrak{Z}^{A}(\mathfrak{r}) \mathfrak{E}^{\prime}\left(\mathfrak{a}_{\mathfrak{R}}\right)$. Since $\mathfrak{r}$ is interior to $a_{\Re} \vee b_{\Re}$ by virtue of Theorem 28 , the basic ring $A$ contains an element $c=c_{\Re}$ with the property that $\mathfrak{r} \epsilon c^{\prime{ }^{\prime}}=c_{\Re}{ }^{\prime \prime}{ }^{\prime}<\left(a_{\Re} \vee b_{\Re}\right)^{\prime-\prime}$. We therefore have $\mathfrak{X}^{A}(\mathfrak{r}) \subset \mathbb{E}(c), \mathfrak{Z}^{A}(\mathfrak{r})=f^{-1}\left(\mathfrak{X}^{A}(\mathfrak{r})\right) \subset f^{-1}(\mathfrak{E}(c))=\mathbb{E}\left(c_{\mathfrak{R}}\right)$. Now the relations $\left(a_{\Re} \vee b_{\Re}\right)^{\prime}-^{\prime} \equiv a_{\Re} \vee b_{\Re}\left(\bmod a_{\Re}\right), c_{\Re}^{\prime}{ }^{\prime} \equiv c_{\Re}\left(\bmod a_{\Re}\right)$, and $c_{\Re}^{\prime}{ }^{\prime \prime}<\left(a_{\Re} \vee b_{\Re}\right)^{\prime-1}$ imply the equivalent relations $\left(a_{\Re} \vee b_{\Re}\right) c_{\Re} \equiv c_{\Re}\left(\bmod a_{\Re}\right),\left(a_{\Re} \vee b_{\Re}\right)^{\prime} c_{\Re} \equiv 0$ $\left(\bmod a_{\Re}\right), \quad \mathbb{F}^{\prime}\left(a_{\Re} \vee b_{\Re}\right) \mathbb{E}\left(c_{\Re}\right) \subset \mathbb{E}\left(\mathfrak{a}_{\Re}\right)$, and $\quad \mathbb{E}\left(c_{\Re}\right) \mathbb{F}^{\prime}\left(\mathfrak{a}_{\Re}\right) \subset \mathbb{E}\left(a_{\Re} \vee b_{\Re}\right) \mathbb{F}^{\prime}\left(\mathfrak{a}_{\Re}\right)$. Thus we obtain the contradiction

$$
\mathfrak{Z}^{A}(\mathfrak{r}) \mathfrak{F}^{\prime}\left(\mathfrak{a}_{\Re}\right) \subset \mathbb{E}\left(c_{\Re}\right) \mathfrak{F}^{\prime}\left(\mathfrak{a}_{\Re}\right) \subset \mathbb{E}\left(a_{\Re} \vee b_{\Re}\right) \mathfrak{F}^{\prime}\left(\mathfrak{a}_{\Re}\right) \subset \mathbb{F}\left(a_{\Re} \vee b_{\Re}\right) .
$$

We conclude therefore that $\mathfrak{B}^{A}(\mathfrak{r}) \mathfrak{E}^{\prime}\left(\mathfrak{a}_{\mathfrak{R}}\right)=\mathfrak{X}^{A}(\mathfrak{r}) \mathfrak{E}^{\prime}\left(\mathfrak{a}_{\mathfrak{R}}\right)$, as we wished to do.

Since no set $\mathfrak{X}^{A}(\mathfrak{r})$ is contained in $\mathfrak{F}(\mathfrak{a})$, as shown in Theorem 28 , the closed sets $\mathfrak{X}^{A}(\mathfrak{r}) \mathfrak{F}^{\prime}(\mathfrak{a})=\mathfrak{Y}^{A}(\mathfrak{r})$ are non-void and constitute a family $\Upsilon^{A}$ in $\mathfrak{F}^{\prime}(\mathfrak{a})$ to which Theorem 14 is applicable. By Theorems 3 and 4 we know that $\mathbb{F}^{\prime}(\mathfrak{a})$ is a bicompact Boolean subspace of $\mathbb{E}(A)$, the sets $\mathbb{E}(a) \mathbb{E}^{\prime}(\mathfrak{a})$ constituting a basis for it. If $\mathfrak{p}$ is any point of this subspace and if $a$ is an element of $A$ such that $\mathfrak{p} \in \mathbb{E}(a) \mathbb{F}^{\prime}(\mathfrak{a})$, it is clear from Theorem 28 that there exists a set $\mathfrak{X}^{A}(\mathfrak{r})$ contained in $\mathfrak{E}(a)$. We therefore have $\mathfrak{Y}^{A}(\mathfrak{r})=\mathfrak{X}^{A}(\mathfrak{r}) \mathfrak{F}^{\prime}(\mathfrak{a}) \subset \mathbb{E}(a) \mathbb{E}^{\prime}(\mathfrak{a})$. Hence 
the family $\mathfrak{Y}^{A}$ is densely distributed in $\mathfrak{F}^{\prime}(\mathfrak{a})$ in accordance with Definition 4. If we consider $\Upsilon^{A}$ as a $T_{0}$-space in accordance with Theorem 14, we wish to show that it is topologically independent of $A$. We therefore compare $\Upsilon^{A}$ for arbitrary $A$ with the particular space $\Upsilon$ for $A=A_{\Re}$. From (3) above we see that the correspondence $\mathfrak{Y}(\mathfrak{r}) \longleftrightarrow \mathfrak{Y}^{A}(\mathfrak{r})$ carries $\Upsilon$ into $\Upsilon^{A}$ in a biunivocal manner, even though we may have $\mathfrak{Y}(\mathfrak{r})=\mathfrak{Y}(\mathfrak{Z})$ or $\mathfrak{Y}^{A}(\mathfrak{r})=\mathfrak{Y}^{A}(\mathfrak{Z})$ when $\mathfrak{r} \neq \mathfrak{z}$. We observe next that the subfamilies of $\Upsilon$ specified by the relations $\mathfrak{V}(\mathfrak{r}) \subset \mathbb{E}\left(a_{\Re}\right) \mathbb{E}^{\prime}\left(\mathfrak{a}_{\Re}\right), a_{\Re}=a \epsilon A$, constitute a basis in $\Upsilon$. If $\mathbb{S}$ is any relatively open subset of $\mathbb{E}^{\prime}\left(\mathfrak{a}_{\mathfrak{R}}\right)$ and if $\mathfrak{Y}(\mathfrak{r})$ is contained in $\mathbb{B}$, we wish to establish the existence of such an element $a_{\Re}$ with the property that $\mathfrak{Y}(\mathfrak{r}) \subset \mathbb{E}\left(a_{\Re}\right) \mathbb{F}^{\prime}\left(\mathfrak{a}_{\Re}\right) \subset \mathfrak{B}$ : Since $\mathfrak{X}^{A}(\mathfrak{r})$ is, by Theorem 26 , the intersection of all the sets $\mathbb{E}(a)$ containing it, the set $\mathfrak{Z}^{A}(\mathfrak{r})=f^{-1}\left(\mathfrak{X}^{A}(\mathfrak{r})\right)$ is the intersection of all the corresponding sets $f^{-1}(\mathfrak{F}(a))=\mathfrak{E}\left(a_{\Re}\right)$; and $\mathfrak{Y}(\mathfrak{r})=\mathfrak{Z}^{A}(\mathfrak{r}) \mathfrak{F}^{\prime}\left(\mathfrak{a}_{\Re}\right)$ is the intersection of all the corresponding sets $\mathbb{E}\left(a_{\Re}\right) \mathbb{E}^{\prime}\left(\mathfrak{a}_{\Re}\right)$. Let us denote by $\mathfrak{F}$ the closed set which is the complement of $(\mathfrak{B})$ in $\mathbb{E}^{\prime}\left(\mathfrak{a}_{\Re}\right)$, and by $\mathfrak{b}$ the class of all elements $a_{\Re}=a \epsilon A$ such that $\mathfrak{X}^{A}(\mathfrak{r}) \subset \mathbb{E}(a)$. If we had $\mathbb{E}\left(a_{\Re}^{(1)}\right) \cdots\left(\mathfrak{E}\left(a_{\Re}^{(n)}\right) \mathfrak{E}^{\prime}\left(\mathfrak{a}_{\Re}\right) \mathfrak{F} \neq 0\right.$ for every choice of $a_{\Re}{ }^{(1)}, \cdots, a_{\Re}{ }^{(n)}$ in $\mathfrak{b}$, the bicompactness of $\mathbb{E}^{\prime}\left(\mathfrak{a}_{\Re}\right)$ would permit us to write

$$
\mathfrak{V}(\mathfrak{r}) \mathfrak{F}=\mathfrak{Z}^{A} \cdot(\mathfrak{r}) \mathfrak{E}^{\prime}\left(\mathfrak{a}_{\mathfrak{R}}\right) \mathfrak{F}=\prod_{a_{\mathfrak{R}} \in \mathfrak{b}} \mathfrak{F}\left(a_{\mathfrak{R}}\right) \mathfrak{F}^{\prime}\left(\mathfrak{a}_{\mathfrak{R}}\right) \mathfrak{F} \neq 0,
$$

contrary to hypothesis. Hence we have $\mathbb{E}\left(a_{\Re}\right) \mathbb{F}^{\prime}\left(\mathfrak{a}_{\Re}\right) \mathfrak{F}=\mathbb{E}\left(a_{\Re}{ }^{(1)}\right) \ldots$ $\mathbb{E}\left(a_{\Re}^{(n)}\right) \mathbb{E}^{\prime}\left(a_{\Re}\right) \mathfrak{F}=0$ for $a_{\Re}=a_{\Re}^{(1)} \cdots a_{\Re}^{(n)}$ and appropriate elements $a_{\Re}^{(1)}, \cdots, a_{\Re}^{(n)}$ in $\mathfrak{b}$. Since we have $\mathbb{E}(a)=\mathbb{E}\left(a^{(1)}\right) \ldots \mathfrak{E}\left(a^{(n)}\right) \supset \mathfrak{X}^{A}(\mathfrak{r})$ where $a=a^{(1)} \cdots a^{(n)}$ and $a^{(1)}, \cdots, a^{(n)}$ are the elements $a_{\Re}^{(1)}, \cdots, a_{\Re}^{(n)}$, considered as elements of $A$, we see that $a_{\Re}=a$ is a member of $\mathfrak{b}$. The desired relation $\mathfrak{Y}(\mathfrak{r}) \subset \mathfrak{E}\left(a_{\mathfrak{R}}\right) \mathfrak{E}^{\prime}\left(\mathfrak{a}_{\mathfrak{R}}\right) \subset \mathfrak{B}$ is thereby established. Since the relations $\mathfrak{V}(\mathfrak{r}) \subset \mathbb{E}\left(a_{\mathfrak{R}}\right) \mathbb{E}^{\prime}\left(\mathfrak{a}_{\mathfrak{R}}\right)$ and $\mathfrak{Y}^{A}(\mathfrak{r}) \subset \mathfrak{E}(a) \mathfrak{E}^{\prime}(\mathfrak{a})$ are equivalent by $(3)$ above when $a_{\Re}=a \epsilon A$, we see that the basis just found for $\Upsilon$ is carried by the biunivocal correspondence $\mathfrak{V}(\mathfrak{r}) \longleftrightarrow \mathfrak{V}^{A}(\mathfrak{r})$ into a class of subfamilies in $\Upsilon^{A}$ which is known from Theorem 23 to be a basis for $\Upsilon^{A}$. Hence the spaces $\Upsilon$ and $\Upsilon^{A}$ are topologically equivalent; and $\Upsilon^{A}$ is topologically independent of $A$.

By reference to Theorem 18, we now see that the correspondence $\mathfrak{X}(\mathfrak{r}) \rightarrow \mathfrak{Y}(\mathfrak{r})$ from $X$ to $\Upsilon$ is a univocal continuous correspondence. Thus the spaces $\Re, \Re^{*}$ equivalent to $X$ and $\Upsilon$ respectively have the property that $\Re^{*}$ is a continuous image of $\Re$. By reference to Theorem 28 , we see that, when $\Re$ is an $H$-space and $\mathfrak{r}$ and $\mathfrak{Z}$ are distinct, $\mathfrak{Y}(\mathfrak{r})$ and $\mathfrak{Y}(\mathfrak{z})$ are disjoint. Hence $\Upsilon$ and $\Re^{*}$ are $H$-spaces in accordance with Theorem 23 ; and the correspondences between $X$ and $\Upsilon, \Re$ and $\Re^{*}$ are biunivocal. To determine the relation of the character of $\Re^{*}$ to that of $\Re$, we proceed as follows. If $\Re$ has infinite character $\mathfrak{c}$, then there exists a basis for $\Re$ with cardinal number $\mathfrak{c}$. 
The Boolean ring $A$ with unit $\Re$ generated by this basis is a basic ring for $\Re$ with cardinal number $c$. The Boolean space $\mathbb{E}(A)$ then has character $c$ by virtue of Theorem 1 . The character of the subspace $\mathbb{E}^{\prime}(\mathfrak{a})$ therefore does not exceed that of $\&(A)$ or that of $\Re$. The space $\Upsilon^{A}$ has character not exceeding that of $\mathfrak{F}^{\prime}(\mathfrak{a})$, as shown in Theorem 23. Since $\Re^{*}$ is equivalent to $\Upsilon^{A}$, we conclude that its character does not exceed the character $\mathfrak{c}$ of $\Re$. Finally the suppression of the open set $\mathbb{E}(\mathfrak{a})$ from the map $m\left(\Re, \mathbb{E}(A), X^{A}\right)$ yields the map $m\left(\Re^{*}, \mathbb{E}^{\prime}(\mathfrak{a}), \Upsilon^{A}\right)$ in accordance with Definition 7; and the latter map is irredundant in accordance with Definition 9 and Theorem 20.

The preceding theorem raises several questions which we shall state and consider later, in Chapter III.

3. Relations between algebraic and other maps. In various applications of the theory of maps, it is essential to have information about the relations of general Boolean maps to the algebraic Boolean maps of the preceding section. The analysis of such relations appears to be quite difficult. In any case we have not succeeded in outlining a comprehensive survey of the possible connections. We shall therefore confine ourselves in the present section to the consideration of a few special results which find immediate application in the sequel. These results are concerned with a new concept in the theory of maps, introduced now by the following definition.

DefINITION 12. If $X$ is any non-void family of distinct non-void closed sets $\mathfrak{X}$ in a $T_{1}$-space $\mathfrak{S}$, then a set $\mathfrak{F}$ in $\mathfrak{S}$ is said to be an $X$-set when

(1) $\mathfrak{F}$ is closed and non-void; (2) every open set \&5 in $\mathbb{S}$ which contains $\mathfrak{F}$ also contains some member of the family $X$.

An $X$-set is said to be minimal (with respect to the property $P$ ) if it is an $X$-set (with property $P$ ) and contains no proper $X$-subset (with property $P$ ).

It is obvious that every member of the family $X$ is an $X$-set; but the chief interest of the definition lies in the possibility that there exist $\mathcal{X}$-sets not belonging to $X$. We may begin by considering the determination of $X$-sets when $\subseteq$ is a bicompact Boolean space.

THEOREM 30. If the space $\subseteq$ of Definition 12 is a bicompact Boolean space (E $(A)$ representing a Boolean ring $A$ with unit, the open sets \& of that definition may be restricted to be bicompact without modifying the content of the definition. If $\mathrm{c}$ is the class of all elements $a$ in $A$ such that $\mathfrak{E}(a)$ contains a given $X$-set $\mathfrak{F}$, then $\mathrm{c}$ has the properties

(1) if $a_{1}, \cdots, a_{n}$ are in $\mathrm{c}$, then $a_{1} \cdots a_{n}$ is in $\mathrm{c}$;

(2) if $a_{1}, \cdots, a_{n}$ are in $\mathrm{c}$, then $\mathbb{E}(a)$, where $a=a_{1} \cdots a_{n}$, contains some member of $X$;

and the relation $\mathfrak{F}=\prod_{a \in c}(\mathbb{E}(a)$ is valid. Conversely, if $\mathrm{c}$ is any non-void subclass of $A$ with the properties (1) and (2), then the set $\mathfrak{F}=\prod_{a \in c} \mathbb{E}(a)$ is an $X$-set. 
If the open set $\mathbb{F}$ contains the closed set $\mathfrak{F}$ in $\mathfrak{S}=\mathbb{F}(A)$, then there exists an element $a$ in $A$ such that $\mathfrak{F} \subset \mathbb{E}(a) \subset \mathbb{B}$. In fact, if $\mathbb{B}$ is any point of $\mathfrak{F}$, there exists an element $a(\mathfrak{z})$ in $A$ such that $\mathfrak{z} \in \mathbb{E}(a(\mathfrak{z})) \subset(B)$; and the bicompactness of $\mathfrak{F}$ permits us to take $a=a\left(\mathbb{B}_{1}\right) \vee \cdots \vee a\left(\mathbb{z}_{n}\right), \mathbb{E}(a)=\mathbb{E}\left(a\left(\mathbb{B}_{1}\right)\right) \mathbf{u} \cdots$ u $\mathbb{E}\left(a\left(\mathbb{z}_{n}\right)\right)$ for a suitable choice of points $\mathbb{B}_{1}, \cdots, \mathbb{B}_{n}$ in $\mathfrak{F}$. Hence the restriction imposed by requiring that $\mathbb{F}=\mathbb{E}(a)$ in Definition 12 does not modify the content of the definition. If $\mathfrak{c}$ is the class of all elements $a$ such that $\mathfrak{F}(a) \supset \mathfrak{F}$, then the properties (1) and (2) are easily established as follows: if $a=a_{1} \cdots a_{n}$ where $a_{1}, \cdots, a_{n}$ are in $\mathfrak{c}$, then $\mathbb{E}(a)=\mathbb{E}\left(a_{1}\right) \cdots\left(\mathbb{E}\left(a_{n}\right) \supset \mathfrak{F}\right.$; thus $a$ is in $\mathfrak{c}$ and $\mathbb{E}(a)$ contains a member of $\mathcal{X}$ since it contains the $\mathcal{X}$-set $\mathfrak{F}$. If $z$ is any point which does not belong to $\mathfrak{F}$, the open set $\mathbb{B}=\mathbb{F}(A)-\{z\}$ contains $\mathfrak{F}$; and there exists an element $a$, necessarily in $\mathfrak{c}$, such that $\mathfrak{F} \subset \mathbb{E}(a) \subset \mathfrak{F}(A)-\{\mathbb{z}\}$. Hence we see that $\mathfrak{F}=\prod_{a \in \mathfrak{E}} \mathfrak{E}(a)$. We pass now to the converse. The properties (1) and (2) assumed for the class $c$ show immediately that when $a_{1}, \cdots, a_{n}$ are in $c$ the set $\mathbb{E}\left(a_{1}\right) \cdots \&\left(a_{n}\right)=\mathbb{E}\left(a_{1} \cdots a_{n}\right)$ is non-void. Since $\mathbb{E}(A)$ is bicompact and the sets $\&(a)$ are closed, the intersection $\prod_{a \in c} \mathfrak{E}(a)$ is a non-void closed set $\mathfrak{F}$. If $\mathbb{E}(b) \supset \mathfrak{F}$, where $b$ is an arbitrary element in $A$, we must have $\&\left(a_{1}\right) \ldots \mathscr{E}\left(a_{n}\right) \xi^{\prime}(b)=0$ for some elements $a_{1}, \cdots, a_{n}$ in $\mathrm{c}$ : for otherwise we would have

$$
0=\mathfrak{F}^{\prime}(b)=\prod_{a \in \mathcal{C}} \mathfrak{E}(a) \mathfrak{F}^{\prime}(b) \neq 0 .
$$

Hence there exists an element $a=a_{1} \cdots a_{n}$ in $c$ such that $\mathfrak{F} \subset \mathbb{E}(a) \subset \mathbb{E}(b)$. Property (2) shows that $\&(a)$, and hence also $\&(b)$, contains some member of $X$. Since $b$ was subject only to the restriction $\mathscr{E}(b) \supset \mathfrak{F}$, it follows that $\mathfrak{F}$ is an $X$-set in accordance with Definition 12 and the first part of the present theorem.

We next consider the existence of minimal $X$-sets.

THEOREM 31. Let $P$ be a property such that the members of any descending transfinite sequence of sets with the property $P$ contain a common subset with the property $P$. Then every set with the property $P$ contains a minimal set with the property $P$.

Let $\Omega$ be the first ordinal number such that the class of ordinals $\alpha<\Omega$ has cardinal number exceeding that of the class of all sets with the property $P$. We then define a "minimizing" sequence $\mathfrak{F}_{\alpha}, \alpha<\Omega$, of sets with the property $P$ such that: (1) $\alpha>\beta$ implies $\mathfrak{F}_{\alpha} \subset \mathfrak{F}_{\beta}$; (2) if $\prod_{\beta<\alpha} \mathfrak{\mho}_{\beta}$ contains a set which has the property $P$ but which is not minimal, then $\mathfrak{F}_{\alpha}$ is a proper subset of one such set. We choose $\mathfrak{F}_{1}$ as an arbitrary set with the property $P$. Then if $\mathfrak{F}_{\beta}$ has been defined for $\beta<\alpha<\Omega$, we form the intersection $\prod_{\beta<\alpha} \mathfrak{F}_{\beta}$. By hypothesis there exists a set $\mathfrak{F}$ which has the property $P$ and is contained in this 
intersection. Now if every such $\mathfrak{F}$ is minimal, we put $\mathfrak{F}_{\alpha}=\mathfrak{F}$; and otherwise we choose $\mathfrak{F}_{\alpha}$ as a proper subset of some $\mathfrak{F}$ so that it has the property $P$. By the principle of transfinite induction $\mathfrak{F}_{\alpha}$ is thereby defined for $\alpha<\Omega$. Now the class of ordinals $\alpha$ such that $\mathfrak{F}_{\alpha}=\mathfrak{F}_{\gamma}$ for some ordinal $\gamma$ in the range $\alpha<\gamma<\Omega$ is a non-void class by virtue of our choice of $\Omega$. This class therefore has a first member $\beta$. The relations $\beta<\beta+1 \leqq \gamma, \mathfrak{F}_{\beta}=\mathfrak{F}_{\gamma}$, imply $\mathfrak{F}_{\beta} \supset \mathfrak{F}_{\beta+1} \supset \mathfrak{F}_{\gamma} \supset \mathfrak{F}_{\beta}$, $\mathfrak{F}_{\beta}=\mathfrak{F}_{\beta+1}$. Since

$$
\prod_{\alpha<\beta+1} \mathfrak{F}_{\alpha}=\prod_{\alpha \leqq \beta} \mathfrak{F}_{\alpha}=\mathfrak{F}_{\beta},
$$

the relation $\mathfrak{F}_{\beta}=\mathfrak{F}_{\beta+1}$ implies in accordance with (2) that $\mathfrak{F}_{\beta+1}$ is minimal. Since it is evident that $\mathfrak{F}_{\beta+1} \subset \mathfrak{F}_{1}$, the theorem is established.

THEOREM 32. If the space $\subseteq$ of Definition 12 is a bicompact $H$-space, then each of the following properties:

(1) the property of being an $X$-set;

(2) the property of being an $X$-set containing a given point 8 ;

(3) the property of being an $X$-set contained in a given set; is a property $P$ of the kind described in the preceding theorem. Hence minimal sets with respect to each of them exist in accordance with that theorem.

Let $P$ be any one of the three properties. Then it is sufficient for us to show that if $\mathfrak{F}_{\alpha}, \alpha<\omega$, is a transfinite sequence such that (1) $\mathfrak{F}_{\alpha}$ has the property $P$, and (2) $\mathfrak{F}_{\alpha} \subset \mathfrak{F}_{\beta}$ when $\alpha>\beta$, then $\prod_{\alpha<\omega} \mathfrak{F}_{\alpha}$ also has the property $P$. Since $\mathfrak{F}_{\alpha}$ is an $X$-set, it is closed and non-void. Hence, by the assumption concerning $\mathfrak{S}$, the intersection $\prod_{\alpha<\omega \mathfrak{F}_{\alpha}}$ is also closed and non-void. In order to show that this set has the required property, it is enough to prove that it is an $X$-set. If $B$ is any open set containing it, we have

$$
\prod_{\alpha<\omega} \mathfrak{F}_{\alpha} \mathfrak{S}^{\prime}=\left(\mathcal{S}^{\prime} \prod_{\alpha<\omega} \mathfrak{F}_{\alpha}=0\right.
$$

where the sets $\mathfrak{F}_{\alpha}\left(\mathfrak{S}^{\prime}\right.$ are closed and satisfy the relation $\mathfrak{F}_{\alpha}\left(\mathfrak{S}^{\prime} \subset \mathfrak{F}_{\beta}\left(\mathfrak{S}^{\prime}\right.\right.$ when $\alpha>\beta$. Our assumption concerning $\subseteq$ now implies that there exists a first ordinal number $\alpha$ preceding $\omega$ for which $\mathfrak{F}_{\alpha} \mathfrak{S}^{\prime}=0$ or, equivalently, $\mathfrak{F}_{\alpha} \subset \mathbb{S}$. The fact that $\mathfrak{F}_{\alpha}$ is an $X$-set shows that (S) contains some member of $\mathcal{X}$. Since the open set $\&$ was subject only to the restriction that it contain

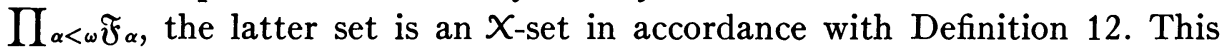
completes the proof.

We may give without proof the following useful result.

TheOREM 33. If the families $X$ and $\Upsilon$ in the space $\subseteq$ satisfy the relation $X \supset \Upsilon$, then every $\Upsilon$-set is an $X$-set; and, if $\subseteq$ is a bicompact $H$-space, every 
minimal $\Upsilon_{- \text {-set contains a minimal }} \mathcal{X}_{\text {-set, every }} \Upsilon_{\text {-set }}$ minimal with respect to the property of containing a given point $z$ contains an $X$-set minimal with respect to the same property.

Naturally, it is of considerable interest to determine the $X$-sets and minimal $\mathcal{X}$-sets in the case of the family $\mathcal{X}$ in an algebraic map. We find the following situation.

Theorem 34. If $m(\Re, \mathfrak{B}, \mathcal{X})$ is an algebraic Boolean map, then the $\mathcal{X}$-sets are characterized as the closed subsets of $\mathscr{B}=\mathbb{E}(A)$ which have at least one point in common with the set $\mathbb{F}^{\prime}(\mathfrak{a})$, where a is the ideal of nowhere dense sets in the basic ring $A$. The minimal $\mathcal{X}$-sets are characterized as the one-element subsets of $\mathbb{E}^{\prime}(\mathfrak{a})$. The $X$-sets minimal with respect to the property of containing a given point $\mathfrak{p}$ in $\mathbb{E}(\mathfrak{a}) \subset \mathfrak{B}=\mathbb{E}(A)$ are characterized as the sets containing two points, the point $\mathfrak{p}$ and a point of $\mathbb{E}^{\prime}(\mathfrak{a})$.

First, let us show that every $\mathcal{X}$-set $\mathfrak{F}$ has at least one point in common with $\mathbb{F}^{\prime}(\mathfrak{a})$. By Theorem 30 , we have $\mathfrak{F}=\prod_{a \in c} \mathfrak{E}(a)$ where $\mathfrak{c}$ is the class of all $a$ in $A$ for which $\mathfrak{F} \subset \mathbb{E}(a)$. By virtue of the bicompactness of $\mathfrak{B}=\mathbb{E}(A)$, we see that

$$
\mathfrak{F} \mathfrak{F}^{\prime}(\mathfrak{a})=\prod_{a \in \mathfrak{c}} \mathfrak{E}(a) \mathfrak{F}^{\prime}(\mathfrak{a})=0
$$

if and only if $\mathbb{E}(a) \xi^{\prime}(\mathfrak{a})=0$ for some $a$ in c. By Theorem 28, we know that $\mathbb{E}(a)$ contains a member of $\mathcal{X}$ if and only if $\mathbb{E}(a) \mathbb{F}^{\prime}(\mathfrak{a}) \neq 0$. Since $\mathfrak{F}$ is an $X$-set, the relation $\mathfrak{F} \subset \mathbb{E}(a)$ implies that $\mathbb{E}(a)$ contains some member of $\mathcal{X}$. Hence we conclude that $\mathfrak{F} \mathfrak{E}^{\prime}(\mathfrak{a}) \neq 0$, as we wished to prove. If $\mathfrak{p}$ is any point of $\mathbb{E}^{\prime}(\mathfrak{a})$, then $\mathfrak{p} \in \mathbb{E}(a)$ implies that $\mathbb{E}(a)$ contains some member of $\mathcal{X}$; and the one-element set $\{\mathfrak{p}\}$ is therefore an $X$-set. From these facts, the remaining statements of the theorem can be deduced in an obvious way.

As a consequence of this theorem, we have the following comment on Theorem 29.

THEOREM 35. In the notation of Theorem 29 , every $Z^{A}$-set in $\mathbb{E}\left(A_{\Re}\right)$ is an $x$-set.

If $\mathfrak{F}$ is a $Z^{A}$-set and $\mathfrak{F}(a) \supset \mathfrak{F}$ where $a \epsilon A_{\Re}$, then $\mathfrak{F}(a)$ contains some set $\mathfrak{Z}^{A}(\mathfrak{r})$. Since $\mathfrak{Z}^{A}(\mathfrak{r}) \supset \mathfrak{X}(\mathfrak{r})$, it follows that $\mathfrak{F}$ is an $\mathcal{X}$-set.

We now proceed to study a natural method for relating a general Boolean map to an algebraic map.

THEOREM 36. Let $m(\Re, \mathfrak{B}, \mathcal{X})$ be an arbitrary Boolean map where the bicompact Boolean space $\mathfrak{B}$ is the representative of a Boolean ring $A$. Let $a^{*}=\mathbb{S}(a)$ be the open subset of $\Re$ determined from the given map through the relation 
$\mathfrak{X}(\mathfrak{r}) \subset \mathbb{E}(a)$, where $a$ is an arbitrary element of $A$. Let $A^{*}$ be the basic ring generated by the basis of all sets $a^{*}=\mathfrak{B}(a)$ in $\Re$; and let $m\left(\Re, \mathfrak{B}^{*}, X^{*}\right)$ be the algebraic map defined by $A^{*}$ in $\mathfrak{B}^{*}=\mathbb{E}\left(A^{*}\right)$. Let $\mathfrak{F}$ be the class of all $X$-sets in $\mathfrak{B}, \mathfrak{F}^{*}$ the class of all $X^{*}$-sets in $\mathfrak{B}^{*}$. If $\mathfrak{F}$ is an arbitrary $X$-set and $\mathrm{c}$ is the class of all a in $A$ such that $\mathbb{E}(a) \supset \mathfrak{F}$, then the set $f(\mathfrak{F})=\prod_{a \in c} \mathbb{E}\left(a^{*}\right)$ is an $X^{*}$-set. The correspondence $\mathfrak{F} \rightarrow f(\mathfrak{F})$ has the properties:

(1) f carries $\mathcal{F}$ univocally into a subclass $\mathfrak{F}^{* *}$ of $\mathfrak{F}^{*}$;

(2) $\mathfrak{F}_{1} \supset \mathfrak{F}_{2}$ implies $f\left(\mathfrak{F}_{1}\right) \supset f\left(\mathfrak{F}_{2}\right)$;

(3) the intersection of $X$-sets with the property $f(\mathfrak{F})=\mathfrak{F}^{*}$, where $\mathfrak{F}^{*}$ is a fixed member of $\mathfrak{F}^{* *}$, is an $X$-set with this property; and the intersection of all sets with the property is the unique $X$-set minimal with respect to this property;

(4) $\mathfrak{X}(\mathfrak{r})$ is the minimal $X$-set with the property $f(\mathfrak{F})=\mathfrak{X}^{*}(\mathfrak{r})$;

(5) $f$ carries the minimal sets in $\mathcal{F}$ biunivocally into the minimal sets in $\mathcal{F}^{* *}$. If the class $\mathfrak{F}^{* *}$ contains the minimal sets in $\mathfrak{F}^{*}$ or, more generally, if each point of the set $\mathfrak{E}^{\prime}\left(\mathfrak{a}^{*}\right)$, where $\mathfrak{a}^{*}$ is the ideal of nowhere dense sets in $A^{*}$, belongs to exactly one minimal set in $\mathfrak{F}^{* *}$, the map $m(\Re, \mathfrak{B}, X)$ has the following properties:

(1) the union of all minimal $X$-sets is an $X$-set $\mathfrak{F}(X)$;

(2) every $X$-set has at least one point in common with the set $\mathfrak{F}(X)$;

(3) if $\mathfrak{E}(a) \subset \mathfrak{F}^{\prime}(\mathcal{X})$, then $\mathbb{E}(a)$ contains no member of $X$. Since the set $\mathfrak{F}(\mathcal{X})$ is closed, it is bicompact.

The preliminary justification of the constructions leading to the algebraic map described here can be obtained by reference to Theorems 14, 23, and 25 and to Definitions 7, 10, and 11 . We begin our proof by a consideration of the definition of the correspondence $f$. By Theorem 30, we have $\mathfrak{F}=\prod_{a \in c} \mathbb{E}(a)$, the notations being those introduced above. The elements $a^{*}=\$(a)$ where $a \epsilon$ constitute a class $\mathrm{c}^{*}$ in $A^{*}$ which has properties (1) and (2) of Theo-

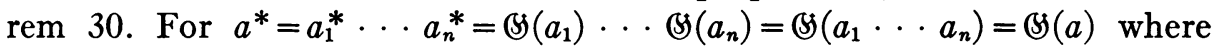
$a=a_{1} \cdots a_{n}$ is in $\mathfrak{c}$; and $a^{*}=\mathfrak{S}(a), a \in \mathfrak{c}$, implies the existence of a point $\mathfrak{r}$ in $\Re$ satisfying the equivalent relations $\mathfrak{X}(\mathfrak{r}) \subset \mathbb{E}(a), \quad \mathfrak{r} \in(S)(a), \quad \mathfrak{r} \epsilon\left(a^{*}\right)^{\prime-\prime}=a^{*}$, $\mathfrak{X}^{*}(\mathfrak{r}) \subset \mathbb{E}\left(a^{*}\right)$. Hence Theorem 30 shows that $f(\mathfrak{F})=\prod_{a \in c} \mathfrak{E}\left(a^{*}\right)$ is an $X^{*}$-set. Properties (1) and (2) of the correspondence $f$ are obvious from the definition. Next we consider property (3). If $A$ is an abstract class of elements $\alpha$ to each of which corresponds a member $\mathfrak{F}_{\alpha}$ of $\mathfrak{F}$ such that $f\left(\mathfrak{F}_{\alpha}\right)=\mathfrak{F}^{*} \epsilon \mathfrak{F}^{* *}$, we wish first to prove that $\mathfrak{F}=\prod_{\alpha \in \mathrm{A}} \mathfrak{F}_{\alpha}$ is an $\mathcal{X}$-set with the property $f(\mathfrak{F})=\mathfrak{F}^{*}$. We again appeal to Theorem 30 . We begin by writing $\mathfrak{F}_{\alpha}=\prod_{\text {eec }(\alpha)} \&(a)$, where $\mathrm{c}(\alpha)$ is the class of all $a$ in $A$ such that $\mathbb{E}(a) \supset \mathfrak{F}_{\alpha}$. We then define $\mathfrak{c}$ as the class of all elements $a=a_{1} \cdots a_{n}$ where $a_{k} \in \mathfrak{c}\left(\alpha_{k}\right)$ for $k=1, \cdots, n$ and $n=1,2$, $3, \cdots$. It is then evident that $\mathrm{c}$ has property (1) of Theorem 30 . In addition 
we can prove that $\mathrm{c}$ has property (2). We first take the arbitrary element $a$ in $\mathfrak{c}$ and express it in the form $a=a_{1} \cdots a_{n}$, where $a_{k} \in \mathcal{c}\left(\alpha_{k}\right)$, noted above.

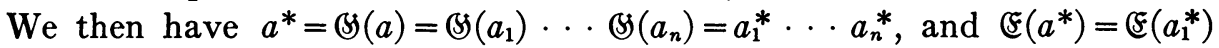
... $\mathbb{E}\left(a_{n}^{*}\right)$. By hypothesis $\mathbb{E}\left(a_{k}^{*}\right) \supset f\left(\mathfrak{F}_{\alpha_{k}}\right)=\mathfrak{F}^{*}$ for $k=1, \cdots, n$. Hence $\mathbb{E}\left(a^{*}\right)$ also contains $\mathfrak{F}^{*}$. Since $\mathfrak{F}^{*}$ is an $\mathcal{X}^{*}$-set, there exists a point $\mathfrak{r}$ in $\Re$ satisfying the equivalent relations $\mathfrak{X}^{*}(\mathfrak{r}) \subset \mathbb{E}\left(a^{*}\right), \mathfrak{r} \epsilon\left(a^{*}\right)^{\prime-^{\prime}}=a^{*}=\mathfrak{S}(a), \mathfrak{X}(\mathfrak{r}) \subset \mathbb{E}(a)$. Since $c$ thus has properties (1) and (2) of Theorem 30, we conclude that

$$
\mathfrak{F}=\prod_{\alpha \in \mathrm{A}} \mathfrak{F}_{\alpha}=\prod_{\alpha \in \mathrm{A}} \prod_{a \in \mathcal{C}(\alpha)} \mathfrak{E}(a)=\prod_{a \in \mathcal{C}} \mathfrak{E}(a)
$$

is an $X$-set. As we saw above, $a \in \mathfrak{c}$ implies $\mathscr{E}\left(a^{*}\right) \supset \mathfrak{F}^{*}$; and it follows that $f(\mathfrak{F}) \supset \mathfrak{F}^{*}$. On the other hand the relation $\mathfrak{F} \subset \mathfrak{F}_{\alpha}, \alpha \epsilon \mathrm{A}$,implies $f(\mathfrak{F}) \subset f\left(\mathfrak{F}_{\alpha}\right)=\mathfrak{F}^{*}$. Hence we have $f(\mathfrak{F})=\mathfrak{F}^{*}$, as we wished to prove. It is now evident that the intersection of all sets $\mathfrak{F}$ such that $f(\mathfrak{F})=\mathfrak{F}^{*}$ is the unique $\mathcal{X}$-set minimal with respect to this property. Part of the property (5) follows immediately from (2) and (3). If $\mathfrak{F}^{*}$ is minimal in the family $\mathfrak{F}^{* *}$, then the set $\mathfrak{F}$ in $\mathfrak{F}$ which is minimal with respect to the property $f(\mathfrak{F})=\mathfrak{F}^{*}$ exists by (3); and it must be minimal in the entire family $\mathscr{F}$, since an $\mathcal{X}$-set $\mathfrak{F}_{1}$ contained in $\mathfrak{F}$ has the properties $f\left(\mathfrak{F}_{1}\right) \subset f(\mathfrak{F})=\mathfrak{F}^{*}, f\left(\mathfrak{F}_{1}\right)=\mathfrak{F}^{*}, \mathfrak{F}_{1} \supset \mathfrak{F}$, and therefore coincides with $\mathfrak{F}$. The rest of property (4) is established by reasoning similar to that applied to prove (3). If $\mathfrak{F}$ is minimal in $\mathfrak{F}$, and $\mathfrak{F}_{1}^{*}$ is a member of $\mathfrak{F}^{* *}$ contained in $\mathfrak{F}^{*}=f(\mathfrak{F})$, then there exists a set $\mathfrak{F}_{1}$ in $\mathfrak{F}$ such that $f\left(\mathfrak{F}_{1}\right)=\mathfrak{F}_{1}^{*}$. We let $\mathfrak{c}$ be the class of all elements $a$ in $A$ such that $a=b b_{1}$ where $\&(b) \supset \mathfrak{F}, \mathbb{E}\left(b_{1}\right) \supset \mathfrak{F}_{1}$. Then $\mathfrak{F} \mathfrak{F}_{1}=\prod_{a \in c}\left(\mathfrak{F}(a)\right.$. If $a=b b_{1}$ is any element in $\mathfrak{c}$, we have $a^{*}=b^{*} b_{1}^{*}$, $\mathfrak{E}\left(a^{*}\right)=\mathbb{E}\left(b^{*}\right) \mathfrak{E}\left(b_{1}^{*}\right)$. By hypothesis $\mathbb{E}\left(a^{*}\right) \supset \mathfrak{F}_{1}^{*}$. Since $\mathfrak{F}_{1}^{*}$ is an $\mathcal{X}^{*}$-set, there exists a point $\mathfrak{r}$ in $\Re$ satisfying the equivalent relations $\mathfrak{X}^{*}(\mathfrak{r}) \subset \mathbb{E}\left(a^{*}\right)$, $\mathfrak{r} \epsilon\left(a^{*}\right)^{\prime{ }^{\prime}}=a^{*}=\mathbb{S}(a), \mathfrak{X}(\mathfrak{r}) \subset \mathbb{E}(a)$. Since $\mathfrak{c}$ thus has properties (1) and (2) of Theorem 30 , we conclude that $\mathfrak{F} \mathfrak{F}_{1}$ is an $\mathcal{X}$-set. By virtue of the fact that $\mathfrak{F}$ is a minimal $X$-set, we must have $\mathfrak{F}_{\mathfrak{F}_{1}}=\mathfrak{F}, \mathfrak{F}_{1} \supset \mathfrak{F}, f\left(\mathfrak{F}_{1}\right) \supset f(\mathfrak{F}), \mathfrak{F}_{1}^{*} \supset \mathfrak{F}^{*}$, and $\mathfrak{F}_{1}^{*}=\mathfrak{F}^{*}$. Thus $\mathfrak{F}^{*}=f(\mathfrak{F})$ is minimal in $\mathfrak{F}^{* *}$, as we wished to prove. If $\mathfrak{F}_{1}$ and $\mathfrak{F}_{2}$ are minimal $X$-sets such that $f\left(\mathfrak{F}_{1}\right)=f\left(\mathfrak{F}_{2}\right)$, then by $(3) \mathfrak{F}_{1} \mathfrak{F}_{2}$ is an $X$-set. Consequently $\mathfrak{F}_{1} \mathfrak{F}_{2}=\mathfrak{F}_{1}, \mathfrak{F}_{1} \mathfrak{F}_{2}=\mathfrak{F}_{2}$ and $\mathfrak{F}_{1}=\mathfrak{F}_{2}$. Thus $f$ defines a biunivocal correspondence between the minimal sets in $\mathcal{F}$ and those in $\mathfrak{F}^{* *}$. This completes the proof of property (5). To prove property (4) we use Theorem 30 once again. We have $\mathfrak{X}(\mathfrak{r})=\prod_{a e c}(\mathfrak{E}(a)$, where $\mathfrak{c}$ is the class of all $a$ in $A$ such that $\mathfrak{X}(\mathfrak{r}) \subset \mathbb{E}(a)$. Since $\mathfrak{X}(\mathfrak{r}) \subset \mathbb{E}(a), \mathfrak{r} \in \mathfrak{S}(a)=a^{*}=\left(a^{*}\right)^{\prime-\prime}$, and $\mathfrak{X}^{*}(\mathfrak{r}) \subset \mathbb{E}\left(a^{*}\right)$ are equivalent relations, we see that

$$
f(\mathfrak{X}(\mathfrak{r}))=\prod_{a \in \mathfrak{c}} \mathfrak{E}\left(a^{*}\right)=\mathfrak{X}^{*}(\mathfrak{r}),
$$


with appropriate reference to Theorem 26 . On the other hand, if $\mathfrak{F}$ is any $X$-set with the property $f(\mathfrak{F})=\mathfrak{X}^{*}(\mathfrak{r})$, we express $\mathfrak{F}$ as an intersection of sets $\mathfrak{E}(a)$ containing $\mathfrak{F}$. We then have $a^{*}=\mathfrak{S}(a), \mathfrak{E}\left(a^{*}\right) \supset \mathfrak{X}^{*}(\mathfrak{r})$ for such elements $a$, in accordance with the definition of the correspondence $f$. The second of these relations implies $\mathfrak{r} \epsilon\left(a^{*}\right)^{\prime-\prime}=a^{*}=\mathfrak{S}(a), \mathfrak{X}(\mathfrak{r}) \subset \mathbb{E}(a)$. Hence $\mathfrak{F}$ contains $\mathfrak{X}(\mathfrak{r})$; and $\mathfrak{X}(\mathfrak{r})$ is the unique $X$-set minimal with respect to the property $f(\mathfrak{F})=\mathfrak{X}^{*}(\mathfrak{r})$.

Finally, we come to the special property described in the last part of the theorem. We begin by considering the class c of all elements $a$ in $A$ such that $\mathbb{E}\left(a^{*}\right)$, where $a^{*}=\Im(a)$, contains a given point $\mathfrak{p}$ in $\mathbb{E}^{\prime}\left(\mathfrak{a}^{*}\right)$. If $a_{1}, \cdots, a_{n}$ are in c, then the element $a=a_{1} \cdots a_{n}$ has the properties $a^{*}=\mathbb{S}(a)=\mathbb{S}\left(a_{1}\right) \cdots$ B $\left(a_{n}\right)$ $=a_{1}^{*} \cdots a_{n}^{*}, \mathbb{E}\left(a^{*}\right)=\mathbb{E}\left(a_{1}^{*}\right) \cdots \mathbb{E}\left(a_{n}^{*}\right), \mathfrak{p} \in \mathbb{E}\left(a^{*}\right)$, and therefore belongs to $\mathrm{c}$. If $a$ is any element in $\mathfrak{c}$, then the relation $p \in \in \mathbb{E}\left(a^{*}\right)$ implies the existence of a point $\mathfrak{r}$ in $\Re$ such that $\mathfrak{X}^{*}(\mathfrak{r}) \subset \mathbb{E}\left(a^{*}\right)$, by virtue of the fact that $\{\mathfrak{p}\}$ is an $X^{*}$-set in accordance with Theorem 34 . Since $\mathfrak{X}^{*}(\mathfrak{r}) \subset \mathbb{E}\left(a^{*}\right)$ implies $\mathfrak{r} \epsilon\left(a^{*}\right)^{\prime}-^{\prime}=a^{*}=\mathfrak{S}(a)$ and $\mathfrak{X}(\mathfrak{r}) \subset \mathbb{E}(a)$, we conclude that $c$ has properties (1) and (2) of Theorem 30. Hence the set $\mathfrak{F}=\prod_{a \in c} \mathfrak{F}(a)$ is an $\mathcal{X}$-set, and its correspondent $\mathfrak{F}^{*}=f(\mathfrak{F})=\prod_{a \in c} \mathfrak{E}\left(a^{*}\right)$ contains the point $\mathfrak{p}$. If $\mathfrak{F}_{1}$ is any $\boldsymbol{X}$-set such that $p \in f\left(\mathfrak{F}_{1}\right)=\mathfrak{F}_{1}^{*}$, we have

$$
\mathfrak{F}_{1}=\prod_{a \in \mathrm{C}_{1}} \mathfrak{E}(a), \quad \mathfrak{F}_{1}^{*}=\prod_{a \in \mathrm{C}_{1}} \mathfrak{F}\left(a^{*}\right) \supset \prod_{a \in \mathrm{C}} \mathfrak{F}\left(a^{*}\right)=\mathfrak{F}^{*},
$$

where $\mathfrak{c}_{1}$ is the class of all $a$ in $A$ such that $\mathfrak{F}_{1} \subset \mathbb{E}(a)$. Hence we see that the set $\mathfrak{F}^{*}$ is the unique set in $\mathfrak{F}^{* *}$ minimal with respect to the property of containing the given point $\mathfrak{p}$. Thus if a minimal set in $\mathfrak{F}^{* *}$ contains the point $\mathfrak{p}$, it must coincide with $\mathfrak{F}^{*}$. We now assume that each point $\mathfrak{p}$ in $\mathfrak{F}^{\prime}\left(\mathfrak{a}^{*}\right)$ belongs to exactly one minimal set in $\mathfrak{F}^{* *}$. Let us consider the union $\mathfrak{F}(X)$ of all minimal sets in $\mathfrak{F}$. If $\mathbb{z}$ is any point in $\mathfrak{F}^{\prime}(X)$, a familiar argument shows that every minimal $X$-set $\mathfrak{F}$ determines an element $a$ in $A$ such that $\mathfrak{F} \subset \mathfrak{F}(a)$, $B \in \mathbb{F}^{\prime}(a)$. The corresponding element $a^{*}=\mathfrak{S}(a)$ then has the property $\mathfrak{F}\left(a^{*}\right) \supset f(\mathfrak{F})=\mathfrak{F}^{*}$. By property (5) above, $\mathfrak{F}^{*}$ is a minimal set in $\mathfrak{F}^{* *}$. By our assumption concerning the minimal sets in $\mathfrak{F}^{* *}$, and by further reference to property (5), we see that the sets $\mathbb{E}\left(a^{*}\right)$ thus obtained cover $\mathbb{E}^{\prime}\left(\mathfrak{a}^{*}\right)$. Because $\mathbb{E}^{\prime}\left(\mathfrak{a}^{*}\right)$ is bicompact, there exist elements $a_{1}, \cdots, a_{n}$ such that the corresponding sets $\mathfrak{E}\left(a_{1}^{*}\right), \cdots, \mathfrak{F}\left(a_{n}^{*}\right)$ cover $\mathfrak{F}^{\prime}\left(\mathfrak{a}^{*}\right)$. If $\mathfrak{F}^{*}$ is any minimal set in $\mathfrak{F}^{* *}$, it contains a point $\mathfrak{p}$ in $\mathbb{E}^{\prime}\left(\mathfrak{a}^{*}\right)$; and this point belongs to no other minimal set in $\mathfrak{F}^{* *}$. If $p \in \mathbb{E}\left(a_{k}^{*}\right)$, a relation which must be satisfied for some index $k$, $k=1, \cdots, n$, we see that $\mathfrak{F}^{*} \subset \mathbb{E}\left(a_{k}^{*}\right)$ in accordance with the results proved above. Furthermore, there exists a unique minimal $\mathcal{X}$-set $\mathfrak{F}$ such that $f(\mathfrak{F})=\mathfrak{F}^{*}$. As we saw above, it is necessary that $\mathfrak{F} \subset \mathbb{F}\left(a_{k}\right)$ : for the set $\prod_{a \in c} \mathbb{E}(a)$, where $\mathfrak{p} \in \mathbb{E}\left(a^{*}\right)$ characterizes the class $\mathfrak{c}$, has $\mathfrak{F}^{*}$ as its correspondent 
and is contained in $\&\left(a_{k}\right)$. Now this result shows that every minimal $X$-set is contained in some of the sets $\&\left(a_{k}\right), k=1, \cdots, n$. Hence, if we put $a(\mathfrak{z})=a_{1} \vee \cdots \vee a_{n}$, we have $\mathfrak{F}(\mathcal{X}) \subset \mathbb{E}(a(\mathfrak{z})), \mathbb{z} \in \mathbb{F}^{\prime}(a(\mathfrak{z}))$. The set $\mathfrak{F}(X)$ therefore coincides with the intersection of the closed sets $\mathbb{E}(a(\mathbb{Z}))$ where $\mathbb{B} \in \mathfrak{F}^{\prime}(X)$; accordingly it must be closed and bicompact. Since $\mathfrak{F}(\mathcal{X})$ contains $\mathcal{X}$-sets, namely, the minimal $X$-sets, it is obviously itself an $X$-set. Since every $X$-set contains a minimal $\mathcal{X}$-set, the remaining properties stated in the theorem are obvious.

In order to apply the preceding theorem, we shall appeal to the following result.

THEOREM 37. Let $\subseteq$ be any non-void subspace of a $T_{0}$-space $\Re$; let $m(\Re, \mathfrak{B}, X)$ be the complete algebraic map of $\Re$ in $\mathfrak{B}=\mathbb{E}\left(A_{\Re}\right)$; and let $\mathfrak{B}(\mathfrak{S})$ be the closure of the union of all sets $\mathfrak{X}(\mathbb{B})$ where $\boldsymbol{z} \in \subseteq$. Then the Boolean map defined by the family $X(\subseteq)$ of all $\mathfrak{X}(\mathbb{B})$, where $\mathbb{B} \in \subseteq$ is a map $m(\mathfrak{S}, \mathfrak{B}(\mathfrak{S}), X(\mathfrak{S})$ ) with the special property described in Theorem 36.

If $B$ is an arbitrary open subset of $\mathfrak{S}$, there exists an open set $a$ in $A_{\Re}$ such that $a \mathfrak{S}=(\mathfrak{B}$. It is then clear that, for $\mathbb{s}$ in $\subseteq$, we have $\mathfrak{X}(\mathfrak{z}) \subset \mathbb{E}(a)$ if and only if $\boldsymbol{\varepsilon} \in \mathbb{G}$. The set $\mathbb{E}(a) \mathfrak{B}(\mathfrak{S})$ is a bicompact open subset of $\mathfrak{B}(\mathfrak{S})$ and therefore represents an element $b$ of the Boolean ring $A$ which has $\mathscr{B}(\subseteq)$ as its representative. The correspondent $b^{*}$ of $b$ in the basic ring $A^{*}$, constructed for $\mathfrak{S}$ as described in Theorem 36 , is seen to coincide with the given set $(5$. Hence the basic ring $A^{*}$ contains every open set in $\subseteq$ as an element. If $\mathfrak{a}^{*}$ is the ideal of nowhere dense sets in $A^{*}$ and if $\mathfrak{p}$ and $\mathfrak{q}$ are distinct points in $\mathbb{E}^{\prime}\left(\mathfrak{a}^{*}\right)$, there exists an element $a^{*}$ in $A^{*}$ such that $\&\left(a^{*}\right)$ contains $\mathfrak{p}$ but not $\mathfrak{q}$. If $b^{*}$ is the interior of $a^{*}$ relative to $\mathfrak{S}$, then $b^{*}$ is also in $A^{*}$. Furthermore, the relation $a^{*} \equiv b^{*}\left(\bmod \mathfrak{a}^{*}\right)$ implies the relation $\mathfrak{E}\left(a^{*}\right) \mathfrak{F}^{\prime}\left(\mathfrak{a}^{*}\right)=\mathfrak{F}\left(b^{*}\right) \mathfrak{F}^{\prime}\left(\mathfrak{a}^{*}\right)$. Hence $\mathbb{E}\left(b^{*}\right)$ contains $\mathfrak{p}$ but not $\mathfrak{q}$. By the previous remarks, the open set $b^{*}$ in $\subseteq$ corresponds to an element $b$ in $A$ through the relation $b^{*}=\mathbb{S}(b)$ of Theorem 36. We can now conclude that the intersection of all sets $\&\left(b^{*}\right)$ which contain $\mathfrak{p} \in \mathfrak{E}^{\prime}\left(\mathfrak{a}^{*}\right)$ and which correspond to sets $b$ in $A$ has no point in common with $\xi^{\prime}\left(a^{*}\right)$ other than the given point. As we saw in the last part of the proof of Theorem 36 , this intersection is a set $\mathfrak{F}^{*}$ belonging to $\mathfrak{F}^{* *}$. To complete the proof of the present theorem we need only show that $\mathfrak{F}^{*}$ is a minimal set in $\mathfrak{F}^{* *}$. We know that $\mathfrak{F}^{*}$ contains a minimal set $\mathfrak{F}_{1}^{*}$ in $\mathfrak{F}^{* *}$ : for, if $\mathfrak{F}$ is an $X(\subseteq)$-set in the map $m(\mathfrak{S}, \mathfrak{B}(\mathfrak{S}), X(\mathfrak{S})$ ), it contains a minimal set $\mathfrak{F}_{1}$; and its correspondent $\mathfrak{F}_{1}^{*}=f\left(\mathfrak{F}_{1}\right)$ is a minimal set belonging to $\mathfrak{F}^{* *}$ and contained in $\mathfrak{F}^{*}$. Since the set $\mathfrak{F}^{*}$ is an $X^{*}$-set it must contain a point of $\mathbb{F}^{\prime}\left(\mathfrak{a}^{*}\right)$ by Theorem 34 . Since $\mathfrak{F}^{*}$ has only the point $\mathfrak{p}$ in common with $\mathbb{F}^{\prime}\left(\mathfrak{a}^{*}\right)$, we see that $\mathfrak{F}_{1}^{*}$ also contains $\mathfrak{p}$. Now $\mathfrak{F}^{*}$ is by construction the set in $\mathfrak{F}^{* *}$ mini- 
mal with respect to the property of containing $\mathfrak{p}$. Hence $\mathfrak{F}^{*}$ coincides with $\mathfrak{F}_{1}{ }^{*}$, and is minimal in $\mathfrak{F}^{* *}$.

4. Applications to the theory of extensions. One of the interesting and difficult problems of general set-theoretic topology is the study of the extensions of a given space. The term "extension" is used here in the sense indicated by the following definition.

Definition 13. If a $T_{0}$-space $\mathfrak{Q}$ contains a subspace $\Re_{\mathfrak{Q}}$ equivalent to a given $T_{0}$-space $\Re$, then $\mathfrak{Q}$ is said to be an extension of $\Re$; and $\Re$ is said to be imbedded in $\mathfrak{Q}$ as the subspace $\Re_{\mathfrak{Q}}$. If $\Re_{\mathfrak{Q}}$ is a proper subset of $\mathfrak{Q}$, then $\mathfrak{Q}$ is said to be a proper extension of $\Re$; and if $\Re \overline{\mathfrak{Q}}=\mathfrak{Q}$, the space $\mathfrak{Q}$ is said to be an immediate extention of $\Re$.

The problem of extensions falls naturally into two distinct parts. If $\Re$ is imbedded in $\mathfrak{Q}$ as the subspace $\Re_{\mathfrak{Q}}$, then the subspace $\Re_{\mathfrak{Q}}$ is evidently an immediate extension of $\Re$ and a closed subset of $\mathfrak{Q}$. Thus the determination of possible extensions of a given $T_{0}$-space $\Re$ involves, first, the determination of an immediate extension of $\Re$, and, secondly, the determination of a space in which this immediate extension can be imbedded as a closed subset. The second step is one which is obviously more arbitrary than the first, since the local structure of $\mathfrak{Q}$ at points "remote" from $\Re \overline{\mathfrak{Q}}$ can in general be modified in a quite essential way without regard to the properties of $\Re$ or of $\Re_{\mathfrak{Q}}$. Thus the second step becomes most interesting when some additional requirement, say, of connectivity or dimensionality, is laid upon the space $\mathfrak{Q}$. The first step, on the other hand, appeals to the intuition as one which is intimately linked with the structure of the given space $\Re$. Here we shall confine our attention to the first step. As we proceed, we shall see in greater detail how the structure of $\Re$ determines that of its immediate extensions and how the theory of Boolean maps gives us a real insight into the problem under consideration.

In the course of our investigations we shall find it desirable to classify the immediate extensions of a given space. For convenience, we collect the appropriate definitions here.

Definition 14. An immediate extension $\mathfrak{Q}$ of a $T_{0}$-space $\Re$ is said to be a strict extension of $\Re$, if, when $\Re$ is imbedded in $\mathfrak{Q}$ as the subspace $\Re_{\mathfrak{O}}$, the following property is verified: if $\mathbb{B}$ is any open set in $\mathfrak{Q}$ and $q$ is any point in $(\mathfrak{S}$, there exists an open set $\mathfrak{S}$ in $\mathfrak{Q}$ such that $q \in \mathfrak{S} \subset(5)$ and such that, whenever $\mathfrak{S}^{*}$ differs from $\mathfrak{S}$ by a nowhere dense set contained in $\Re_{\mathfrak{Q}}^{\prime}$, the interior of $\mathfrak{S}^{*}$ is contained in (s).

Definition 15. An extension $\mathfrak{Q}$ of a $T_{0}$-space $\Re$ is said to be a $T_{1}$-extension 
of $\Re$ if, when $\Re$ is imbedded in $\mathfrak{Q}$ as the subspace $\Re_{\mathfrak{Q}}$, the relations $q \in \mathfrak{Q}, \mathfrak{r} \in \Re_{\mathfrak{Q}}^{\prime}$, and $\mathfrak{q} \neq \mathfrak{r}$ imply $\mathfrak{r}_{\leftarrow}\{\mathfrak{q}\}^{-}, \mathfrak{q}\left\{\{\mathfrak{r}\}^{-}\right.$; in other words, that each of the points $\mathfrak{q}, \mathfrak{r}$ is contained in an open subset of $\mathfrak{Q}$ which does not contain the other.

Definition 16. An extension $\mathfrak{O}$ of a $T_{0}$-space $\Re$ is said to be an $H$-extension of $\Re$ if, when $\Re$ is imbedded in $\mathfrak{Q}$ as the subspace $\Re_{\mathfrak{O}}$, the relations $q \in \mathfrak{Q}$, $\mathfrak{r} \in \Re_{\mathfrak{Q}}^{\prime}$, and $\mathfrak{q} \neq \mathfrak{r}$ imply the existence of two disjoint open subsets of $\mathfrak{Q}$ which contain $q$ and $\mathfrak{r}$ respectively.

While the significance of Definitions 15 and 16 is evident, we may comment briefly on Definition 14 . The property on which the latter definition is based means roughly that the points of $\Re_{\mathfrak{Q}}^{\prime}$ are no more "densely" distributed in $\mathfrak{Q}$ than are those of $\Re \mathfrak{Q}$. A strict extension of $\Re$ is therefore one in which the "new" points are not adjoined in too lavish a manner. The technical reasons for introducing the particular form of definition which has just been set forth, will be developed below.

An obvious method for constructing immediate extensions of a given $T_{0}$-space $\Re$ is based on the use of Boolean maps together with the concept of $X$-sets introduced in Definition 12. We obtain the following theorem.

TheORem 38. Let $m(\Re, \mathfrak{B}, X)$ be an arbitrary Boolean map of a $T_{0}$-space $\Re$ in a bicompact Boolean space $\mathfrak{B}$; and let $Z$ be any family of $X$-sets in $\mathfrak{B}$ containing the family $X$. Then under the topology of Theorems 14 and 23, $Z$ is a $T_{0}$-space which is an immediate extension of $\Re$. In order that $Z$ be a $T_{1}$-extension of $\Re$, it is necessary and sufficient that no set in $Z-X$ contain or be contained in any distinct set belonging to $Z$. In order that $Z$ be an $H$-extension of $\Re$, it is sufficient that no set in $Z-X$ have a point in common with any distinct set l $e$ longing to $Z$.

If we consider $Z$ and $X$ as topological spaces, it is evident that $X$ is a subspace everywhere dense in $Z$ : for, if $Z_{0}$ is any element of $Z$ and $(S)$ is any open subset of $\mathscr{B}$ containing $Z_{0}$, the neighborhood of $Z_{0}$ specified by the relation $\mathbb{Z} \subset \mathcal{B}$ contains some element $\mathfrak{X}$ of the subspace $X$, by virtue of the fact that $Z_{0}$ is an $X$-set. Since $\Re$ is equivalent to $X$, by the definition of a map, the space $Z$ is an immediate extension of $\Re$. The conditions for $Z$ to be a $T_{1^{-}}$or an $H$-extension of $\Re$ are obtained automatically when one adjusts the argument used in Theorem 23 to the requirements of Definitions 15 . and 16.

In order to show that in Theorem 38 we may restrict attention to the algebraic Boolean maps without any loss of generality, we must establish some algebraic preliminaries.

THEOREM 39. If $\Re$ is a subspace of the $T_{0^{-}}$space $\mathfrak{Q}$ such that $\Re^{-}=\mathfrak{Q}$ and 
if $A$ is any subring of the complete basic ring $A_{\mathfrak{O}}$, then the correspondence $a \rightarrow a \Re$ carries $A$ homomorphically into a subring $B$ of the complete basic ring $A_{\Re}$. If $\mathfrak{b}$ is the ideal in $A$ defined by the relation a $\Re=0$, then $\mathfrak{b}$ consists of all nowhere dense sets which belong to $A$ and are contained in $\Re^{\prime}$; and $B$ is an isomorph of $A / \mathfrak{b}$. The sets a $\Re$ in $B$ which are nowhere dense relative to $\Re$ are precisely those for which a is nowhere dense relative to $\mathfrak{\Omega}$.

It is evident that the correspondence $a \rightarrow a \Re$ carries $A$ homomorphically into a ring $B$ of subsets of $\Re$; and that $B$ is an isomorph of $A / \mathfrak{b}$ in accordance with $\mathrm{R}$ Theorem 43 . If $a$ does not belong to the ideal $a$ of nowhere dense sets in $A$, its interior $a^{\prime-^{\prime}}$ is a non-void open subset of $a$. Since $\Re^{-}=\mathfrak{Q}$ implies $a^{\prime-^{\prime}} \Re \neq 0$, the set $a \Re$ contains a non-void subset, $a^{\prime-^{\prime}} \Re$, which is open relative to $\Re$. Thus $a \Re$ has interior points relative to $\Re$ and cannot be a nowhere dense subset of $\Re$. On the other hand, if $a$ is nowhere dense relative to $\mathfrak{Q}$, we can show that $a \Re$ is nowhere dense relative to $\Re$. The closure of $a \Re$ relative to $\Re$ is $(a \Re)-\Re$, the complement of this set relative to $\Re$ is $(a \Re)^{-\prime} \Re$, and the closure of the latter set relative to $\Re$ is $\left[(a \Re)^{-\prime} \Re\right]-\Re$. We therefore have to prove that $\left[(a \Re)^{-\prime} \Re\right]^{-} \Re=\Re$. In view of the relations $(a \Re)^{-} \Re \subset a^{-} \Re$, $(a \Re)^{-\prime} \Re \supset a^{-\prime} \Re$, it is evidently sufficient to prove that $\left(a^{-\prime} \Re\right)^{-}=\mathfrak{Q}$. Now our assumption that $a$ is nowhere dense means that $a^{--}=\mathfrak{Q}$. If we denote by $(\mathfrak{S}$ the open set $a^{-\prime}$ in $\mathfrak{Q}$, we can complete our demonstration by deducing $(\mathfrak{S} \Re)^{-}=\mathfrak{Q}$ from the known relations $\mathfrak{S}^{-}=\Re^{-}=\mathfrak{Q}$, $\mathfrak{B}^{\prime}-=\mathfrak{S}^{\prime}$. Since

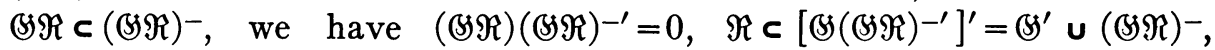

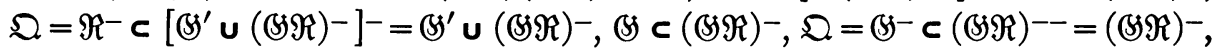
and hence $(\mathfrak{R})^{-}=\mathfrak{Q}$, as we desired to show. Thus we see that, when $a \in A$, the set $a \Re$ is nowhere dense relative to $\Re$ if and only if $a \in \mathfrak{a}$. It follows that the relation $a \equiv a^{\prime \prime^{\prime}}(\bmod \mathfrak{a})$ in $A$ implies the relation $a \Re \equiv a^{\prime-^{\prime}} \Re\left(\bmod \mathfrak{a}_{\Re}\right)$, where $\mathfrak{a}_{\Re}$ is the ideal of all nowhere dense sets in $A_{\Re}$. Since $a^{\prime \sigma^{\prime}} \Re$ is open relative to $\Re$, it is clear that, by definition, $a \Re$ belongs to $A_{\Re}$. The various further properties of $B$ and $\mathfrak{b}$ are now evident.

Theorem 40. Let $\Re, \mathfrak{Q}, A, B$, and $\mathfrak{b}$ have the same meanings as in the preceding theorem; let $B$ have the property that it is a basic ring for $\Re$; let $m(\mathfrak{Q}, \mathfrak{E}(A), \mathcal{Z})$ and $m(\Re, \mathfrak{F}(B), \Upsilon)$ be the algebraic maps determined by $A$ and $B$ respectively; and let $m(\Re, \mathfrak{E}(A), X)$ be the map obtained by suppressing from the family $Z$ in $m(\mathfrak{Q}, \mathfrak{E}(A), Z)$ those members which correspond to points of $\Re^{\prime}$. Then a set in $\mathbb{E}(A)$ is a Z-set if and only if it is an $X$-set. If $A$ contains a basis for $\mathfrak{Q}$, then $B$ contains a basis for $\Re$ and is a basic ring for $\Re$; and the map $m(\Re, \&(B), \Upsilon)$ is equivalent to the map obtained from $m(\Re, \&(A), X)$ by the suppression of the open set $\mathbb{E}(\mathfrak{b})$. Under the same condition on $A$, the set $\mathbb{E}(\mathfrak{b})$ 
is a set of redundancy in the map $m(\mathfrak{Q}, \mathfrak{E}(A), Z)$ if and only if $\Re, \mathfrak{Q}$, and $\mathfrak{b}$ are related in the following manner:

$(\mathrm{P})$ if $\mathbb{S}$ is any open set in $\mathfrak{Q}$ and $\mathfrak{q}$ is any point in $(\mathfrak{B}$, there exists an open set $\mathfrak{S}$ in $\mathfrak{Q}$ such that $q \in \mathfrak{S} \subset(S)$ and such that, whenever $\mathfrak{S}^{*} \equiv \mathfrak{S}(\bmod \mathfrak{b})$, the interior of $\mathfrak{S}^{*}$ is contained in (5).

The property $(\mathrm{P})$ holds whenever $\mathfrak{Q}$ is a strict extension of $\Re$; and $\mathfrak{Q}$ is a strict extension of $\Re$ whenever the property (P) holds for the case where $A$ is the complete basic ring for $\mathfrak{Q}$. When $\mathfrak{E}(\mathfrak{b})$ is a set of redundancy in accordance with the foregoing conditions, its suppression from the map $m(\mathfrak{Q}, \mathbb{E}(A), Z)$ yields a map equivalent to $m(\mathfrak{Q}, \mathfrak{E}(B), \mathfrak{W})$, where $\mathfrak{W}$ is a family of $\Upsilon$-sets containing $\Upsilon$ as a subfamily in the map $m(\Re, \&(B), \Upsilon)$.

If $\mathfrak{F}$ is any $Z$-set in $\mathfrak{F}(A)$ and $\mathfrak{F}(a) \supset \mathfrak{F}$, then there exists a set $\mathbb{Z}$ in $Z$ such that $\mathbb{E}(a) \supset \mathfrak{Z}$. If $q$ is the correspondent of this set in $\mathfrak{Q}$, then $q \in a^{\prime \prime}$ in accordance with Theorem 28. Since $a^{\prime-}$ is open in $\mathfrak{Q}$, the fact that $\Re^{-}=\mathfrak{Q}$ implies the existence of a point $\mathfrak{r}$ in $\Re$ which belongs to $a^{\prime{ }^{\prime}}$. If $\mathfrak{X}$ is the set in $X \subset \mathcal{Z}$ corresponding to the point $\mathfrak{r}$, then $\mathfrak{E}(a) \supset \mathfrak{X}$. Hence the set $\mathfrak{F}$ is an $X$-set in accordance with Definition 12 . Since $X \subset Z$, Theorem 30 shows that every $\mathcal{X}$-set is a $Z$-set. Thus the $\mathcal{X}$-sets are identical with the $Z$-sets.

If $A$ contains a basis in $\mathfrak{Q}$, then $B$ contains a basis in $\Re$ : for the sets in any basis in $\mathfrak{Q}$ intersect $\Re$ in a basis for $\Re$. Thus, in this case, $B$ is a basic ring for $\Re$. In view of the isomorphism between $B$ and the quotient-ring $A / \mathfrak{b}$, the closed set $\mathbb{E}^{\prime}(\mathfrak{b})$ in $\mathbb{E}(A)$ is a Boolean space representing $B$ in accordance with Theorem 4 ; and $\mathfrak{F}^{\prime}(\mathfrak{b})$ and $\mathbb{E}(B)$ are topologically equivalent in such a manner that the relation $b=a \Re$ implies the correspondence of the sets $\mathbb{E}^{\prime}(\mathfrak{b})(\mathfrak{E}(a)$ and $\mathbb{E}(b)$ under the equivalence. In order that $\mathfrak{Z}(\mathfrak{r}) \subset \mathbb{E}(b)$, where $\mathfrak{r} \in \Re$ and $b \epsilon B$, it is necessary and sufficient that $\mathfrak{r}$ be an interior point of $b$ relative to $\Re$. In order that $\mathbb{F}^{\prime}(\mathfrak{b}) \mathfrak{X}(\mathfrak{r}) \subset \mathbb{F}^{\prime}(\mathfrak{b}) \mathfrak{E}(a)$, where $\mathfrak{r} \in \Re$ and $a \epsilon A$, it is necessary and sufficient that $\mathfrak{X}(\mathfrak{r}) \subset \mathfrak{E}(\mathfrak{b}) \cup \mathfrak{E}(a)$ or, equivalently, that $\mathfrak{X}(\mathfrak{r}) \subset \mathfrak{E}(c)$, where $c$ is an element of $A$ such that $c \equiv a(\bmod \mathfrak{b})$. A proof of this assertion runs as follows: the relations $\mathfrak{X}(\mathfrak{r}) \subset \mathfrak{E}(\mathfrak{b}) \cup \mathfrak{E}(a)$ and $\mathfrak{X}(\mathfrak{r}) \mathfrak{F}^{\prime}(a) \subset \mathfrak{E}(\mathfrak{b})$ are equivalent; since $\mathfrak{X}(\mathfrak{r}) \mathfrak{F}^{\prime}(a)$ is a closed set in the bicompact space $\mathbb{E}(A)$, a familiar argument shows that the second of these relations can hold if and only if there exists an element $d$ in $\mathfrak{b}$ such that $\mathfrak{X}(\mathfrak{r}) \mathfrak{E}^{\prime}(a) \subset \mathbb{E}(d)$; if we take $c=a \vee d \equiv a(\bmod \mathfrak{b})$, we have $\mathfrak{X}(\mathfrak{r}) \subset \mathbb{E}(a) \cup \mathbb{E}(d)=\mathbb{E}(c)$; and, on the other hand, if $c \equiv a(\bmod \mathfrak{b})$ and $\mathfrak{X}(\mathfrak{r}) \subset \mathfrak{F}(c)$, we have $d=a^{\prime} c \in \mathfrak{b}, \mathfrak{X}(\mathfrak{r}) \mathbb{F}^{\prime}(a)$ $c \mathbb{E}(c) \mathbb{E}^{\prime}(a)=\mathbb{E}\left(a^{\prime} c\right)=\mathbb{E}(d)$. With the help of the indicated characterizations of the relations $\mathfrak{V}(\mathfrak{r}) \subset \mathfrak{E}(b), \mathfrak{F}^{\prime}(\mathfrak{b}) \mathfrak{X}(\mathfrak{r}) \subset^{\prime} \mathfrak{F}^{\prime}(\mathfrak{b}) \mathfrak{E}(a)$, we shall now show that the equivalence between $\mathfrak{F}^{\prime}(\mathfrak{b})$ and $\mathfrak{E}(B)$ carries $\mathfrak{S}^{\prime}(\mathfrak{b}) \mathfrak{X}(\mathfrak{r})$ into $\mathfrak{V}(\mathfrak{r})$. First, if we have $\mathfrak{F}^{\prime}(\mathfrak{b}) \mathfrak{X}(\mathfrak{r}) \subset \mathfrak{F}^{\prime}(\mathfrak{b})(\mathfrak{F}(a)$, we find an element $c$ of the kind described 
above, noting that $c \equiv a(\bmod \mathfrak{b})$ implies $b=a \Re=c \Re$ by the definition of the ideal $\mathfrak{b}$, and that $\mathfrak{X}(\mathfrak{r}) \subset \mathfrak{F}(c)$ implies $\mathfrak{r} \in c^{\prime-\prime}$. Since $\mathfrak{r}$ is in the set $c^{\prime-\prime} \Re$ which is contained in the interior of $b=c \Re$ relative to $\Re$, we see that $\mathfrak{Y}(\mathfrak{r}) \subset \mathbb{E}(b)$. Now $\mathbb{E}^{\prime}(\mathfrak{b}) \mathfrak{X}(\mathfrak{r})$ is the intersection of all the sets $\mathbb{E}^{\prime}(\mathfrak{b}) \mathfrak{E}(a)$ containing it; its image is therefore the intersection of all the corresponding sets $\mathbb{E}(b)$ in $\mathbb{E}(B)$, where $b=a \Re$; and hence its image contains $\mathfrak{Y}(\mathfrak{r})$. Secondly, if we have $\mathfrak{Y}(\mathfrak{r}) \subset \mathbb{}(b)$ or, equivalently, if $\mathfrak{r}$ is in the interior of $b$ relative to $\Re$, our assumption that $A$ contains a basis for $\mathfrak{Q}$ leads to the result that there exists a set $d=c \Re$, where $c$ is an open set belonging to $A$ and $d$ is therefore open relative to $\Re$, with the properties $\mathfrak{r} \epsilon d<b$. Hence the relations $\mathfrak{r} \epsilon c=c^{\prime}{ }^{\prime}$, $\mathfrak{E}(d) \subset \mathbb{E}(b)$ imply the relations $\mathfrak{X}(\mathfrak{r}) \subset \mathbb{E}(c), \quad \mathbb{F}^{\prime}(\mathfrak{b})\left(\mathfrak{F}(c) \subset \mathbb{F}^{\prime}(\mathfrak{b}) \mathbb{E}(a)\right.$, where $d=c \Re$ and $b=a \Re$. We conclude that $\mathbb{E}^{\prime}(\mathfrak{b}) \mathfrak{X}(\mathfrak{r}) \subset \mathbb{E}^{\prime}(\mathfrak{b}) \mathfrak{E}(a)$. Now since $\mathfrak{Y}(\mathfrak{r})$ is the intersection of all the sets $\mathbb{E}(b)$ containing it, where $b \epsilon B$, its image in $\mathfrak{F}^{\prime}(\mathfrak{b})$ is the intersection of all the corresponding sets $\mathbb{F}^{\prime}(\mathfrak{b}) \mathfrak{F}(a)$, where $b=a \Re$; and its image therefore contains the set $\mathfrak{F}^{\prime}(\mathfrak{b}) \mathfrak{X}(\mathfrak{r})$. Combining these results, we see that the sets $\mathscr{F}^{\prime}(\mathfrak{b}) \mathfrak{X}(\mathfrak{r})$ and $\mathfrak{Y}(\mathfrak{r})$ are images of one another, as we wished to prove. Accordingly, the suppression of the open set $\mathbb{E}(\mathfrak{b})$ from the map $m(\Re, \&(A), X)$ yields a map equivalent to $m(\Re, \&(B), \Upsilon)$. It follows that $\mathfrak{E}(\mathfrak{b})$ is a set of redundancy in harmony with Definitions 8 and 9.

It is now possible to analyze the removal of the set $\mathfrak{E}(\mathfrak{b})$ from the map $m(\mathfrak{Q}, \mathfrak{E}(A), \mathcal{Z})$. Applying the criterion given in Theorem 18 , we see that $\mathfrak{E}(\mathfrak{b})$ is a set of redundancy if and only if, whenever $\mathbb{E}(a) \supset Z_{0}$, there exists a set $a_{0}$ in $A$ such that $\&(\mathfrak{b}) \cup\left(\&\left(a_{0}\right) \supset Z_{0}\right.$ while $\&(\mathfrak{b}) \cup \mathbb{E}\left(a_{0}\right) \supset \mathbb{Z}$ implies $\&(a)>\mathbb{Z}$. Let us consider the sets in $A$ which are congruent $(\bmod \mathfrak{b})$ to such a set $a_{0}$. If $c$ is any such set, we have $\mathbb{E}(\mathfrak{b}) \cup \mathbb{E}\left(a_{0}\right)=\mathbb{E}(\mathfrak{b}) \cup \mathbb{E}(c)$ since $\mathbb{E}\left(a_{0}\right) \Delta \mathbb{E}(c)=\mathfrak{E}\left(a_{0}+c\right) \subset \mathbb{E}(\mathfrak{b})$. As we proved in the preceding paragraph, the relation $\mathbb{E}(\mathfrak{b}) \cup \mathbb{E}\left(a_{0}\right) \supset .3$ is equivalent to the relation $\mathbb{E}(c) \supset 3$ for some such set $c$. Hence it is possible to choose $a_{0}$ so that $\&\left(a_{0}\right) \supset Z_{0}$ while $\mathbb{E}(\mathfrak{b}) \cup \mathbb{E}\left(a_{0}\right) \supset: 3$ implies $\&(a) \supset \mathfrak{Z}:$ for if $a_{0}$ does not have the first property we can replace it by a congruent set which does, and this substitution does not affect the significance of the inclusion $\mathbb{E}(\mathfrak{b}) \cup \mathbb{E}\left(a_{0}\right) \supset \mathbb{Z}$. The point $q$ corresponding to $Z_{0}$ is then interior to $a_{0}$ as well as to $a$. In order that the inclusion $\mathbb{E}(\mathfrak{b}) \cup\left(\mathbb{E}\left(a_{0}\right) \supset Z\right.$ imply $\mathbb{E}(a) \supset \not$, it is necessary and sufficient that $c \equiv a_{0}(\bmod \mathfrak{b})$ imply $c^{\prime-\prime}<a^{\prime-\prime}$. We begin with the necessity of this condition. The sets $c^{\prime-1}, a^{\prime \prime}$ are the subsets of $\mathfrak{Q}$ specified by the relations $\mathbb{E}(c) \supset \mathfrak{Z}, \mathfrak{E}(a) \supset \mathfrak{Z}$. Hence, from the assumptions $c \equiv a_{0}(\bmod \mathfrak{b})$, $\mathbb{E}(c) \supset \mathbb{Z}$, we can deduce the relations $\mathbb{E}(\mathfrak{b}) \cup \mathbb{E}\left(a_{0}\right)=\mathbb{E}(\mathfrak{b}) \cup \mathbb{E}(c) \supset \mathbb{Z}, \mathbb{E}(a) \supset \mathbb{Z}$, and $c^{\prime{ }^{\prime}}<a^{\prime{ }^{\prime}}$. We pass then to the sufficiency. If $\mathbb{E}(\mathfrak{b}) \cup \mathbb{E}\left(a_{0}\right) \supset \mathbb{3}$, there exists a set $c$ in $A$ congruent to $a_{0}(\bmod \mathfrak{b})$ such that $\mathbb{E}(c) \supset \mathbb{Z}$; and the assumption that $c \equiv a_{0}(\bmod \mathfrak{b})$ implies $c^{\prime-^{\prime}}<a^{\prime-\prime}$ then leads to the relation $\mathbb{E}(a) \supset \mathbb{Z}$. In view of the preceding discussion, we see that $\mathfrak{E}(\mathfrak{b})$ is a set of redundancy 
if and only if, whenever $a$ is a set in $A$ and $\mathfrak{q}$ an interior point of $a$, there exists a set $a_{0}$ in $A$ which contains $q$ as an interior point and which has the property that, for $c$ in $A$, the relation $c \equiv a_{0}(\bmod \mathfrak{b})$ implies $c^{\prime-^{\prime}}<a^{\prime-^{\prime}}$. It is evident that if $A$ contains a basis this condition can be replaced by the requirement that $\mathfrak{Q}, \mathfrak{R}$, and $\mathfrak{b}$ have the property $(\mathrm{P})$ stated in the theorem. First, let us deduce (P) from the condition just given. If $B$ is any open set in $\mathfrak{Q}$ and $\mathfrak{q}$ any point of $\mathbb{B}$, we choose $a$ in $A$ so that $q \epsilon a^{\prime \alpha^{\prime}} \subset \mathcal{B}$; we may, of course, take $a$ as an open set if we wish. We then determine $a_{0}$ and choose $\mathfrak{S}$ as an open set in $A$ so that $q \in \mathfrak{S} \subset a_{0}^{\prime-\prime}$. Then,.if $\mathfrak{S}^{*} \equiv \mathfrak{S}(\bmod \mathfrak{b})$, we see that $\mathfrak{S}^{*}$ belongs to $A$ and is contained in the set $c=a_{0} \cup \mathfrak{S}^{\prime} \mathfrak{S}^{*} \equiv a_{0}(\bmod \mathfrak{b})$, where $c$ also is in $A$. Hence we have $\left(\mathfrak{S}^{*}\right)^{\prime-^{\prime}} \subset c^{\prime-^{\prime}} \subset a^{\prime-\prime} \subset(\mathfrak{S )}$. Thus (P) is verified. On the other hand, if $(\mathrm{P})$ holds, we apply it, taking $\mathrm{B}=a^{\prime{ }^{\prime}}$ with $a$ in $A$ and $\mathfrak{q} \in a^{\prime \prime}$, so as to determine an open set $\mathfrak{S}$ containing $\mathfrak{q}$ and possessing the other indicated properties. We then choose $a_{0}$ as an open set in $A$ so that $\mathfrak{q} \epsilon a_{0} \subset \mathfrak{S}$. If $c \equiv a_{0}(\bmod \mathfrak{b})$, we see that $c$ belongs to $A$ and is contained in $\mathfrak{S}^{*}=\mathfrak{S} \cup a_{0}^{\prime} c \equiv \mathfrak{S}(\bmod \mathfrak{b})$. Hence we have $c^{\prime-\prime}<\left(\mathfrak{S}^{*}\right)^{\prime-\prime} \subset \mathfrak{S}=a^{\prime-^{\prime}}$, as we wished to prove.

On comparison with Definition 14, it is evident that the property (P) holds whenever $\mathfrak{Q}$ is a strict extension of $\Re$. On the other hand, if $A$ is the complete basic ring of $\mathfrak{Q}$, the ideal $\mathfrak{b}$ consists of all the nowhere dense subsets of $\Re^{\prime}$; and, if the property (P) holds in this case, $\mathfrak{Q}$ must therefore be a strict extension of $\Re$. The remaining statements of the theorem are obvious consequences of the results already obtained.

On combining Theorems 39 and 40, we see that the construction of extensions of a given space $\Re$ can be carried out in the following way:

THEOREM 41. If $\Re$ is an arbitrary $T_{0}$-space and $A_{\Re}$ is the complete basic ring of $\Re$, then every immediate extension of $\Re$ can be found by the following construction: the algebraic map $m\left(\Re, \&\left(A_{\Re}\right), \Upsilon\right)$ is constructed, the space $\&\left(A_{\Re}\right)$ is then imbedded in any suitable bicompact Boolean space $\mathfrak{B}$, and each set $\mathfrak{Y}$ is enlarged by the adjunction of points in $\mathfrak{B}-\mathfrak{E}\left(A_{\Re}\right)$ to form a set $\mathfrak{X}$ in such a way that the resulting family $X$ in $\mathfrak{B}$ provides a map $m(\Re, \mathfrak{B}, X)$; and then the construction of Theorem 39 is applied to the latter map. In this procedure the following specializations are possible:

(1) every strict extension of $\Re$ can be obtained under the conditions $\mathfrak{B}=\mathbb{E}\left(A_{\Re}\right), X=\Upsilon$

(2) there is no loss of generality in supposing that the enlarged sets $\mathfrak{X}$ corresponding to sets $\mathfrak{Y}$ are disjoint whenever the latter are disjoint;

(3) every immediate $H$-extension can be obtained under the restriction described in (2) and the further restriction that no set 3 in the family 
$Z-X$ have a point in common with any distinct set in the family $Z$, the notations being those of Theorem 39;

(4) every strict $H$-extension can be obtained under the conditions described in (1) and (3), that in (2) being trivial.

The foregoing construction can be applied to any algebraic map $m(\Re, \&(A), \Upsilon)$, but does not necessarily provide all extensions of $\Re$ when $A \neq A_{\Re}$.

If $\mathfrak{Q}$ is any immediate extension of $\Re$, we can apply Theorem 40 using the complete basic ring $A_{\mathfrak{Q}}$ of $\mathfrak{Q}$. The corresponding basic ring $B$ then coincides with the complete basic ring $A_{\Re}$ for $\Re$, as we see by reference to Theorem 39. Indeed, it is evident that $B$ contains every subset of $\Re$ which is open relative to $\Re$, since $A_{\mathfrak{Q}}$ contains every open subset of $\mathfrak{Q}$. Furthermore, if $\mathfrak{F}$ is a subset of $\Re$ nowhere dense relative to $\Re$, we see that $\mathfrak{F}$ is in $A_{\mathfrak{Q}}$ and hence also in $A_{\Re}$ : for the assumed relation $\left(\mathfrak{F}^{-\prime} \Re\right)-\Re=\Re$ implies $\mathfrak{Q}=\mathfrak{R}^{-} \subset\left(\mathfrak{F}^{-\prime} \mathfrak{R}\right)^{-} \subset \mathfrak{F}^{-\prime} \mathfrak{R}^{-}=\mathfrak{F}^{-\prime}$, so that $\mathfrak{F}$ is nowhere dense relative to $\mathfrak{Q}$. According to Theorem 24, it follows that $B$ contains $A_{\Re}$; but, as a subring of $A_{\Re}, B$ coincides with $A_{\Re}$. Hence the map $m\left(\mathfrak{Q}, \mathfrak{E}\left(A_{\mathfrak{Q}}\right), Z\right)$ is related to the map $m\left(\Re, \&\left(A_{\Re}\right), \Upsilon\right)$ in the manner indicated in Theorem 40 and described from another point of view in the constructive program stated above. Thus our construction is capable of providing all possible immediate extensions of $\Re$. If $\mathfrak{Q}$ is a strict extension of $\Re$, we know from Theorem 40 that the set

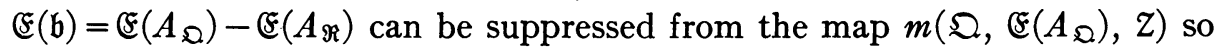
as to yield a map $m\left(\mathfrak{Q}, \mathbb{E}\left(A_{\Re}\right), \mathfrak{W}\right)$ with $\mathscr{W} \supset \Upsilon$. Thus we obtain all strict extensions of $\Re$ under the special conditions stated in (1). If we now refer to the proof of Theorem 28 , we see that two distinct points in $\Re$ (or in $\mathfrak{Q}$ ) have the $H$-separation property, namely, the property of belonging respectively to two disjoint open sets, if and only if their representative sets $\mathfrak{Z}$ (or $\mathbb{Z}$ ) are disjoint. We observe that two distinct points in $\Re$ have the $H$-separation property relative to $\Re$ if and only if they have that property also relative to $\mathfrak{Q}$ : for the open sets in $\Re$ are precisely the sets $\leftrightarrow \Re$ where $\leftrightarrow$ is open in $\mathfrak{Q}$; and the relations $\left(\mathscr{S}_{1} \mathfrak{S}_{2}\right) \Re=\left(\mathfrak{S}_{1} \Re\right)\left(\mathfrak{H}_{2} \Re\right)=0$ and $\mathfrak{H}_{1} \mathfrak{H}_{2}=0$ are equivalent by virtue of the fact that $\Re^{-}=\mathfrak{Q}$. Hence the sets $\mathfrak{X}$ in $m\left(\mathfrak{Q}, \mathfrak{E}\left(A_{\mathfrak{Q}}\right), Z\right.$ ) or $m\left(\Re, \mathfrak{E}\left(A_{\mathfrak{Q}}\right), X\right)$ corresponding to two points in $\Re$ are disjoint if and only if these points have the $H$-separation property relative to $\Re$; that is, if and only if the corresponding sets $\mathfrak{Y}$ in $m\left(\Re, \mathbb{E}\left(A_{\Re}\right), \Upsilon\right)$ are disjoint. Thus we conclude that (2) and (3) are valid, the latter in accordance with Definition 16. Reviewing the preceding discussion, we see also that (4) is valid.

There are two general comments to be made on the construction described above. First, the imbedding of a Boolean space in another offers no difficulty: for, by reference to Chapter I, $\S 3$, we see that this can be accom- 
plished, in all possible ways, by imbedding the given Boolean space as a set $\mathfrak{F}$ in one of the universal Boolean spaces $\mathfrak{B}_{\mathfrak{c}}$ of sufficiently great character $\mathfrak{c}$, and then retaining some Boolean subspace of $\mathfrak{B}_{\mathfrak{c}}$ which contains $\mathfrak{F}$. The whole process can, of course, be expressed in purely algebraic terms. Secondly, the construction of the family $X$ from the family $\Upsilon$ has not been analyzed in a precise way. We see in fact that there is a significant distinction between the concepts of immediate and of strict extensions. The strict extensions of a $T_{0}$-space $\Re$ are obviously quite closely bound by the topological structure of $\Re$, while the immediate extensions are related to $\Re$ in a somewhat vague manner.

The final statement of the theorem does not require elaboration.

As an immediate corollary of Theorem 41 , we have the following result:

Theorem 42. Every immediate $T_{1}$-extension of a $T_{1}$-space is a $T_{1}$-space; and every immediate $H$-extension of an $H$-space is an $H$-space.

We discuss only the second statement of the theorem, leaving the quite similar proof of the first part to the reader. If the $T_{0}$-space $\mathfrak{Q}$ is an $H$-extension of the $H$-space $\Re$, then we see, as in the proof of Theorem 41, that no set in the family $Z-X$ has a point in common with any distinct set in $Z$; and that the sets in $X$, like those in $\Upsilon$, are mutually disjoint. Hence the sets belonging to $Z$ are all disjoint; and $\mathfrak{Q}$ is therefore an $H$-space.

We shall now proceed to consider, with the help of the preceding general theory, several more specific problems concerning extensions. These problems all cluster about the following general definition:

Definition 17. A $T_{0}$-space $\Re$ is said to be absolutely closed with respect to a particular type of extension if it has no proper extension of that type.

The first results which we report are well known and almost trivial.

THEOREM 43. The only $T_{0}$-space which is absolutely closed with respect to immediate extension is the void space.

Using the construction of Theorem 41 , we map a non-void $T_{0}$-space $\Re$ in the space $\&\left(A_{\Re}\right)$, imbed $\&\left(A_{\Re}\right)$ as a proper subset in a bicompact Boolean space $\mathfrak{B}$, take $X=\Upsilon$, and obtain $Z$ by adjoining to $X$ a single $X$-set which has at least one point in common with $\mathfrak{B}-\mathbb{E}\left(A_{\Re}\right)$. The determination of such an $X$-set is easily carried out with the help of Theorem 34 . Under the usual topology, $Z$ is an immediate $T_{0}$-extension of $\Re$. In any $T_{0}$-space the void set is closed, $0^{-}=0$. Hence the void $T_{0}$-space can be imbedded in any $T_{0}$-space and is closed therein. We see therefore that it has no proper immediate $T_{0}$-extension.

We can, however, obtain a stronger result than this. 
THEOREM 44. The only $T_{0}$-space which is absolutely closed with respect to strict $T_{0}$-extension is the void space. Indeed, every non-void $T_{0}$-space $\Re$ becomes, by suitable adjunction of a single point, a bicompact $T_{0}$-space which is a strict $T_{0}$-extension of $\Re$.

Let $\mathfrak{Q}$ be the class obtained by adjoining a single point $\xi$ to the space $\Re$. In $\mathfrak{Q}$, let $\mathfrak{F}$ be the class comprising the following subsets of $\mathfrak{Q}$ : (1) the void set; (2) the sets $\mathfrak{F} \cup\{\xi\}$, where $\mathfrak{F}$ is closed in $\Re$. Then the class $\mathscr{F}$ has the following properties: (1) the finite union and the arbitrary intersection of sets in $\mathcal{F}$ are in $\mathcal{F} ;(2)$ the void set and the set $\mathfrak{Q}$ are both in $\mathcal{F}$. Hence we can introduce in $\mathfrak{Q}$ a closure operation such that the closed sets are precisely the sets belonging to $\mathcal{F}^{*}$ Since this closure operation has the properties that $0^{-}=0$ and $\{\mathfrak{p}\}^{-}=\{\mathfrak{q}\}^{-}$implies $\mathfrak{p}=\mathfrak{q}$, the space $\mathfrak{Q}$ is a $T_{0}$-space. In fact, $0^{-}=0$ is trivial, and the second property is established as follows: if $\mathfrak{p \neq q}$ and $p \in \Re, q \in \Re$, then $\{p\}-$ and $\{q\}^{-}$are obtained by adjoining $\xi$ to the corresponding closures, relative to $\Re$, of $\{p\}$ and $\{q\}$, so that $\{p\}^{-} \neq\{q\}^{-}$; and, if $\mathfrak{p} \in \Re$, then $\{\mathfrak{p}\}^{-}$contains $\{\xi\}^{-}=\{\xi\}$ as a proper subset, the set $\{\xi\}=0 \cup\{\xi\}$ being closed by definition. Moreover, the space $\mathfrak{Q}$ is bicompact. Indeed, if an open set $\mathbb{B}$ in $\mathfrak{Q}$ contains $\xi$, then $\mathfrak{G}^{\prime}$ is closed and does not contain $\xi$, so that $\mathfrak{S}^{\prime}=0$ and $\mathfrak{B}=\mathfrak{Q}$. Hence any family of open sets which covers $\mathfrak{Q}$ must contain a subfamily consisting of $\mathfrak{Q}$ alone, which already covers $\mathfrak{Q}$.

It remains for us to prove that $\mathfrak{Q}$ is a strict extension of $\Re$. The closed sets in $\Re$ are precisely the sets $\mathfrak{F} \Re$ where $\mathfrak{F}$ is closed in $\mathfrak{Q}$, so that $\mathfrak{Q}$ contains $\Re$ as a relative subspace. The closure of $\Re$ in $\mathfrak{Q}$ is the intersection of all closed subsets of $\mathfrak{Q}$ which contain $\Re$; but, since the only such set is $\mathfrak{Q}$ itself, we have $\Re^{-}=\mathfrak{Q}$. Hence $\mathfrak{Q}$ is an immediate extension of $\Re$. The only subsets of $\Re^{\prime}$ are 0 and $\{\xi\}$. Since $0^{-^{-}}=0^{\prime-}=\mathfrak{Q}^{-}=\mathfrak{Q},\{\xi\}^{-^{-}}=\{\xi\}^{\prime-}=\Re^{-}=\mathfrak{Q}$, both sets are nowhere dense in $\mathfrak{Q}$. Thus the only way of modifying an open subset (S) of $\mathfrak{Q}$ by operating with the nowhere dense subsets of $\Re^{\prime}$ is to suppress or adjoin $\xi$. Hence we have to consider two cases under Definition 16: first, the case where $B$ contains $\xi$ and this point is suppressed; and, second, the case where (B) does not contain $\xi$ and this point is adjoined. If $\&$ contains $\xi$, then $\mathfrak{B}=\mathfrak{Q}$ and the open set $\mathfrak{B}-\{\xi\}$ is contained in $\mathbb{S}$. If $B)$ does not contain $\xi$, the interior of $\mathbb{B} \cup\{\xi\}$ coincides with $\mathbb{B}$ : for $(\mathbb{B} \cup\{\xi\})^{\prime-^{\prime}}=\left(\mathbb{S}^{\prime} \Re\right)^{-\prime}$ $=\left(\mathbb{S}^{\prime} \Re \cup\{\xi\}\right)^{\prime}=\left(\mathbb{B} \cup \Re^{\prime}\right) \Re=\mathbb{S}$ since $\mathscr{H}^{\prime} \Re$ is closed in $\Re$ and obviously has $\mathbb{S}^{\prime} \Re \cup\{\xi\}$, a closed set in $\mathfrak{Q}$, as its closure relative to $\mathfrak{O}$. Thus we see that $\mathfrak{Q}$ is a strict extension of $\Re$ in harmony with Definition 16 .

We have also the following fact.

*AH, p. 41, Satz VI. 
THEOREM 45. The only $T_{0}$-spaces which are absolutely closed with respect to immediate $T_{1}$-extension are the finite $T_{0}$-spaces.

While it would no doubt be instructive to discuss this assertion by means of the general mapping theory, the argument is quite involved. It is therefore simpler to appeal to results given by Alexandroff and Hopf. $\dagger$ Their construction shows how to adjoin a single point to an infinite $T_{0}$-space so as to obtain an extension. It is easily verified that this extension is actually an immediate $T_{1}$-extension. On the other hand, if $\mathfrak{Q}$ is a $T_{1}$-extension of a finite $T_{0}$-space $\Re$, we can show that $\Re^{-}=\Re$ in $\mathfrak{Q}$ and can then conclude that $\Re$ has no proper immediate $T_{1}$-extension. In fact if $p \in \Re$ and $q \in \Re^{\prime}$, we see that there exists an

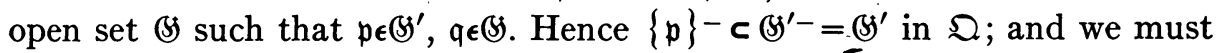
have $\{\mathfrak{p}\}-\subset \Re$, since $q$ is arbitrary in $\Re^{\prime}$. If the points of $\Re$ are $\mathfrak{p}_{1}, \cdots, \mathfrak{p}_{n}$, we therefore have $\Re^{-}=\left\{p_{1}\right\}-\cup \cdots u\left\{p_{n}\right\}-\subset \Re, \Re^{-}=\Re$, as we wished to prove.

In contrast with the foregoing results we shall now establish the existence of less trivial $T_{0}$-spaces which are absolutely closed with respect to strict $T_{1}$-extension.

THEOREM 46. In an arbitrary infinite class $\Re$, let a closure operation be defined as follows: (1) if $\mathfrak{F}$ is a finite subset of $\mathfrak{R}$, then $\mathfrak{F}^{-}=\mathfrak{F}$; (2) if $\mathfrak{F}$ is an infinite subset of $\Re$, then $\mathfrak{F}^{-}=\Re$. Then $\Re$ is a bicompact $T_{1}$-space which is absolutely closed with respect to strict $T_{1}$-extension.

It is easily verified that the indicated closure operation has the properties $\mathfrak{F}^{-} \supset \mathfrak{F}, \mathfrak{F}^{--}=\mathfrak{F}^{-},\left(\mathfrak{F}_{1} \cup \mathfrak{F}_{2}\right)^{--}=\mathfrak{F}_{1}^{-} \cup \mathfrak{F}_{2}^{-}, 0^{-}=0,\{\mathfrak{r}\}^{-}=\{\mathfrak{r}\}$, so that $\Re$ is a $T_{1}$-space. Moreover, $\Re$ is not an $H$-space. Indeed the only closed sets in $\Re$ are $\Re$ and its finite subsets, the only open sets in $\Re$ are the void set and the subsets differing from $\Re$ by finite sets, and any two non-void open sets must therefore have points in common. The nowhere dense sets in $\Re$ are precisely the finite subsets of $\Re$ : for $\mathfrak{F}^{-\prime}=\Re$ if and only if $\mathfrak{F}^{-\prime}$ is infinite, $\mathfrak{F}^{-}$finite, and $\mathfrak{F}$ finite and equal to $\mathfrak{F}^{-}$. The complete basic ring $A_{\Re}$ for $\Re$ is thus seen to consist of the finite subsets of $\Re$ and their complements. In order to determine the Boolean space $\mathbb{E}\left(A_{\Re}\right)$, we first introduce a new topology in the class $\Re$, obtaining a space $\Re^{*}$. The closure operation is defined for this purpose by setting $\mathfrak{F}^{-}=\mathfrak{F}$ for every subset $\mathfrak{F}$ of $\Re$. In $\Re^{*}$ every set is both closed and open; in particular, the one-element sets constitute a basis in $\Re^{*}$. It is therefore evident that $\Re^{*}$ is a non-bicompact Boolean space in which the bicompact subspaces are precisely the finite subsets. Thus $\Re^{*}$ is a representa. tive of the Boolean ring without unit consisting of the finite subsets of $\Re^{*}$ or of $\Re$. Since the ring $A_{\Re}$ is obtained from the one just described by the

$\dagger$ AH, p. 26, Beispiel 3, and p. 90, footnote. 
adjunction of $\Re$ as unit, we conclude that $\mathfrak{E}\left(A_{\Re}\right)$ is obtained from the space $\Re^{*}$ by the suitable adjunction of a single point $\mathfrak{p}$ in accordance with Theorem 8 . Since the ideal $\mathfrak{a}_{\Re}$ of all nowhere dense sets in $A_{\Re}$ coincides with the class of all finite subsets of $\Re$, we see further that the set $\mathfrak{E}^{\prime}\left(\mathfrak{a}_{\Re}\right)$ consists of the point $\mathfrak{p}$ alone. It is now evident that in the map $m\left(\Re, \mathcal{E}\left(A_{\Re}\right), \Upsilon\right)$ the sets $\mathfrak{V}$ are precisely the two-element sets containing the point $\mathfrak{p}:$ if $\mathfrak{r}$ is any point of $\Re$ the sets in $A_{\Re}$ which contain it as an interior point are precisely the infinite sets in $A_{\Re}$ which contain it; and the representatives of these sets in $\mathfrak{F}\left(A_{\Re}\right)$ have as their intersection the set $\mathfrak{Y}(\mathfrak{r})$ consisting of the point $\mathfrak{r}^{*}=\mathfrak{r}$ in $\Re^{*} \subset \mathbb{E}\left(A_{\Re}\right)$ and the adjoined point $\mathfrak{p}$. If we now apply the construction of Theorem 41 to find the strict $T_{1}$-extensions of $\Re$, we find that we must take $Z=X=\Upsilon$ : for the only $\Upsilon$-sets in $\mathbb{E}\left(A_{\Re}\right)$ are those which contain the point $\mathfrak{p}$, by virtue of Theorem 34; and every $\Upsilon$-set therefore either contains a set $\mathfrak{Y}$ or is contained in a set $\mathfrak{Y}$. Thus $\Re$ is absolutely closed with respect to strict $T_{1}$-extension, as we wished to prove. To show that $\Re$ is bicompact, we recall that any non-void open set in $\Re$ differs from $\Re$ by a finite set; and it is evident that any family of open sets which covers $\Re$ contains a finite covering subfamily, one member of this subfamily being chosen arbitrarily and the others then being chosen so as to cover the complementary finite set.

If we consider the map described in the preceding proof, we see that it is possible to state the following result.

THEOREM 47. The space $\Re$ of Theorem 46 has a bicompact strict extension which is a $T_{0}$-space absolutely closed with respect to strict $T_{1}$-extension.

Following the construction of Theorem 41 , we take $\mathfrak{B}=\mathbb{E}\left(A_{\Re}\right)$ and $X=\Upsilon$, and determine $Z$ as the family consisting of the sets in $X$ together with the set $\{\mathfrak{p}\}$. We thus obtain a $T_{0}$-space $\mathfrak{Q}$ which is an immediate extension of $\Re$ arising from $\Re$ by the adjunction of a single point $\xi$ corresponding to the set $\{\mathfrak{p}\}$. From the fact that every set in $X=\Upsilon$ contains $\mathfrak{p}$, we see that $\{\xi\}^{-}=\mathfrak{Q}$. In consequence the only nowhere dense subset of $\Re^{\prime}$ is the void set. Therefore the complete basic rings $A_{\mathfrak{Q}}$ and $A_{\Re}$ are isomorphic under the correspondence $a \rightarrow a \Re$, as we see by reference to Theorem 39 and the proof of Theorem 41. The map $m\left(\mathfrak{Q}, \mathfrak{E}\left(A_{\Re}\right), Z\right)$ which was constructed above is therefore equivalent to the complete algebraic map of $\mathfrak{Q}$. As in the preceding theorem, we infer that $\mathfrak{Q}$ is absolutely closed with respect to strict $T_{1}$-extension. Moreover, we see that $\mathfrak{Q}$ is not merely an immediate extension of $\Re$ but also a strict extension. Finally we show that $\mathfrak{Q}$ is bicompact. Any family of open sets which covers $\mathfrak{Q}$ intersects $\Re$ in a family of open sets which covers $\Re$; and therefore contains a finite subfamily which covers $\Re$, by virtue of the 
bicompactness of $\Re$. To this subfamily, we can adjoin a member of the given family which contains the point $\xi$, thereby obtaining a finite subfamily which covers the entire space $\mathfrak{Q}$.

THEOREM 48. In order that a $T_{0}$-space $\Re$ be absolutely closed with respect to strict $T_{1}$-extension, it is necessary that $\Re$ be bicompact. Any non-bicompact $T_{0}$-space $\Re$ becomes, by the suitable adjunction of a single point, a bicompact $T_{0}$-space $\mathfrak{Q}$; and $\mathfrak{Q}$ is a strict $T_{1}$-extension of $\Re$.

If $\Re$ is a non-bicompact $T_{0}$-space, we define a topology in the class $\mathfrak{Q}=\Re \cup\{\xi\}$ by specifying that the closed subsets of $\mathfrak{Q}$ be (1) the bicompact subspaces of $\Re$ and (2) the sets $\mathfrak{F} \cup\{\xi\}$ where $\mathfrak{F}$ is closed in $\Re$. The indicated subsets clearly have the properties which characterize them as the closed sets under a suitable closure operation.* Since $\{\xi\}^{-}=\{\xi\}$ on account of the fact that $\{\xi\}=\{\xi\} \cup 0$ is closed in $\mathfrak{Q}$, and since $\Re^{-}=\mathfrak{Q}$ on account of the fact that $\mathfrak{Q}$ is the only closed subset of $\mathfrak{Q}$ which contains $\Re$, we see that $\mathfrak{Q}$ is an immediate $T_{1}$-extension of $\Re$ containing $\Re$ as an open subset. It is easily verified that $\mathfrak{Q}$ is a $T_{0}$-space, of course. That $\mathfrak{Q}$ is bicompact, we see as follows: if a family of open sets covers $\mathfrak{Q}$, we choose an arbitrary member $\mathbb{B}$ such that $\xi \in\left(\mathbb{S}\right.$, observing that $\mathfrak{H}^{\prime}$ must be bicompact since it is closed in $\mathfrak{Q}$ and does not contain $\xi$; and we can then determine a finite covering subfamily by selecting from the given family a finite number of further sets which cover the bicompact set $\mathcal{S}^{\prime}$. Finally we show that $\mathfrak{Q}$ is a strict extension of $\Re$. Obviously the nowhere dense subsets of $\Re^{\prime}$ are 0 and $\{\xi\}$. Thus if $(B)$ is any open set containing $\xi$, the modified set $B-\{\xi\}=ß \Re$ is open and is contained in $\mathscr{S}$. If $\mathscr{S}$ is an open set containing a point $\mathfrak{r}$ in $\Re \subset \mathfrak{Q}$, there exists an open set $\mathfrak{S}$ with the properties $\mathfrak{r} \in \mathfrak{S} \subset \mathbb{G}, \xi \in \mathfrak{Q}^{\prime}$ and the special property that $\mathfrak{S}^{\prime} \mathfrak{R}$ is closed but not bicompact relative to $\Re$. We find $\mathfrak{S}$ as follows. First, in the space $\Re$, there must exist an open set $\mathfrak{S}_{0}$ which contains $\mathfrak{r}$ and which has a non-bicompact complement relative to $\Re$ : for otherwise $\Re$ would be bicompact, contrary to hypothesis; indeed, any covering family of open sets would contain a set covering $r$ and a finite number of further sets covering the bicompact complement of the first. Now $\mathfrak{H}_{0}$, being open relative to the open subset $\Re$ of $\mathfrak{Q}$, is open in $\mathfrak{Q}$ and does not contain $\xi$. If we put $\mathfrak{E}=\mathfrak{S}_{0} \mathfrak{B}$,

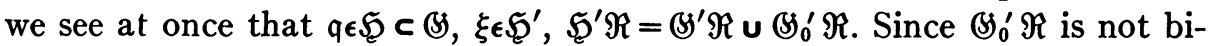
compact, $\mathfrak{S}^{\prime} \Re$ cannot be bicompact. Thus the construction of $\mathfrak{W}$ is completed. We now see that the interior of the modified set $\mathfrak{S} \cup\{\xi\}$ coincides with $\mathfrak{S}$ and is therefore contained in $\mathfrak{S}$ : we have $(\mathfrak{S} \cup\{\xi\})^{\prime-}=\left(\mathfrak{S}^{\prime} \mathfrak{R}\right)^{-}=\mathfrak{S}^{\prime} \mathfrak{R} \cup\{\xi\}$ since $\mathfrak{S}^{\prime} \Re$ is closed in $\Re$ but not bicompact; and thus $(\mathfrak{F} \cup\{\xi\})^{\prime-\prime}=\left(\mathfrak{S}^{\prime} \Re \cup\{\xi\}\right)^{\prime}$

\footnotetext{
* See AH, p. 41, Satz VI, and pp. 93-94.
} 
$=(\mathfrak{S} \cup\{\xi\}) \Re=\mathfrak{E}$. Consequently, $\mathfrak{Q}$ is a strict extension of $\Re$ in accordance with Definition 14.

From the preceding construction, it is apparent that a $T_{0}$-space absolutely closed with respect to strict $T_{1}$-extension is necessarily bicompact. The converse proposition seems not to be true. Examples might be developed on the basis of Theorem 41 to show that such is the case; but we shall not continue the discussion here.

The study of $H$-extensions is somewhat easier because of the special conditions noted in Theorem 41. We first have a general characterization.

THEOREM 49. In order that a $T_{0}$-space $\Re$ be closed with respect to immediate or with respect to strict $H$-extension, it is necessary that in every algebraic map $m(\Re, \mathfrak{B}, X)$ the family $X$ cover the closed set $\mathbb{E}^{\prime}(\mathfrak{a})$, where $\mathfrak{a}$ is the ideal of nowhere dense sets in the basic ring $A$ defining the map; and it is sufficient that in a single algebraic map the family $X$ have this property.

First, let $\mathfrak{Q}$ be an immediate $H$-extension of $\Re$; and consider the maps $m\left(\mathfrak{Q}, \mathfrak{E}\left(A_{\mathfrak{Q}}\right), Z\right), m\left(\Re, \mathfrak{E}\left(A_{\Re}\right), \Upsilon\right)$ as described in Theorem 41 . Since $\mathfrak{Q}$ is an $H$-extension of $\Re$, the sets in $Z-X$, where $\mathcal{X}$ is the family arising from the map $m\left(\Re, \mathbb{E}\left(A_{\bigcirc}\right), X\right)$, are disjoint from the sets of $Z$. Since any set $Z$ is an $X$-set, it must have points in common with the set $\mathbb{E}^{\prime}\left(\mathfrak{a}_{\Re}\right) \subset \mathbb{E}\left(A_{\Re}\right) \subset \mathbb{E}\left(A_{\mathfrak{Q}}\right)$, if we regard $\mathbb{E}\left(A_{\Re}\right)$ for convenience as a subset of $\mathbb{E}\left(A_{\mathfrak{Q}}\right)$ in accordance with the analysis of Theorem 41 . Indeed, we know from Theorem 34 that every set $\mathfrak{Z}$ in $\mathbb{E}\left(A_{\Re}\right)$ has a point in common with $\mathbb{E}^{\prime}\left(\mathfrak{a}_{\Re}\right)$; and from Theorem 41 that every set $\mathfrak{X}$ is obtained from a corresponding set $\mathfrak{Y}$ by the adjunction of points in $\mathbb{E}\left(A_{\mathfrak{Q}}\right)-\mathfrak{E}\left(A_{\Re}\right)$. Thus, if $\mathfrak{F}$ is any $\mathcal{X}$-set in $\mathfrak{E}\left(A_{\mathfrak{Q}}\right)$ and $\mathbb{B}$ any open set containing $\mathfrak{F}$, then $\mathbb{B}$ contains some set $\mathfrak{X}$, hence contains a set $\mathfrak{V}$ which is a subset of $\mathfrak{X}$, and hence contains some point of $\mathcal{F}^{\prime}\left(a_{\mathfrak{R}}\right)$. It follows that $\mathfrak{F} \mathscr{F}^{\prime}\left(\mathfrak{a}_{\Re}\right) \neq 0$, as we wished to show. Thus, if $\mathfrak{Q}$ is a proper extension of $\Re$, the family $Z-X$ contains a set $Z$ which is disjoint from every set $\mathfrak{X}$ and thus contains a point in $\mathscr{F}^{\prime}\left(\mathfrak{a}_{\Re}\right)$ which belongs to no set $\mathfrak{Z}$; in other words, $\Upsilon$ does not cover $\mathbb{F}^{\prime}\left(\mathfrak{a}_{\Re}\right)$.

Next, let the map $m\left(\Re, \mathbb{E}\left(A_{\Re}\right), \Upsilon\right)$ be such that $\Upsilon$ does not cover $\mathbb{E}^{\prime}\left(\mathfrak{a}_{\Re}\right)$; in particular, let $\mathfrak{p}$ be a point of $\mathscr{F}^{\prime}\left(\mathfrak{a}_{\Re}\right)$ which belongs to no set $\mathfrak{Y}$. We then carry out the construction of Theorem 41 , taking $\mathfrak{B}=\mathbb{E}\left(A_{\Re}\right), X=\Upsilon$ and determining $Z$ by adjoining $\{p\}$ to the family $X$. By Theorem $34,\{p\}$ is an $X$-set. We therefore obtain an immediate $H$-extension $\mathfrak{Q}$ of $\Re$ which arises from $\Re$ by the adjunction of a single point $\xi$ corresponding to the set $\{p\}$ in $Z$. It is evident that $\{\xi\}$ is closed but not open in $\mathfrak{Q}$, and that $\Re$ is open but not closed in $\mathfrak{Q}$. Now we can prove that $\mathfrak{Q}$ is a strict extension of $\Re$. Clearly the nowhere dense subsets of $\Re^{\prime}$ are 0 and $\{\xi\}$. If $\&$ is any open set 
containing $\xi$, then the modified set $\xi-\{\xi\}=\Im \Re$ is open and contained in $\$$. If $B$ is any open set containing a point $\mathfrak{r}$ in $\Re$, then the fact that $\mathfrak{r}$ and $\xi$ have the $H$-separation property implies the existence of an open set $\mathfrak{S}$ such that $\mathfrak{r} \in \mathfrak{S C} \subset \mathcal{S}, \xi \in \mathfrak{S}^{-\prime} \subset \mathfrak{S}^{\prime}$. The modified set $\mathfrak{S} \cup\{\xi\}$ cannot contain $\xi$ as an interior point since, if it did, the set $(\mathfrak{S} \cup\{\xi\}) \mathfrak{S}^{-\prime}=\{\xi\}$ would have interior points. Hence the interior of $\mathfrak{S} \cup\{\xi\}$ coincides with $\mathfrak{F}$ and is contained in $\mathfrak{S}$. It follows that $\mathfrak{Q}$ is a strict extension of $\Re$ in accordance with Definition 14 .

If we change our notation slightly to conform with that of Theorem 29, we can therefore assert that the $T_{0}$-space $\Re$ is absolutely closed with respect to immediate or strict $H$-extension if and only if in the complete algebraic map $m\left(\Re, \&\left(A_{\Re}\right), X\right)$ the family $\mathcal{X}$ covers the set $\mathbb{E}^{\prime}\left(\mathfrak{a}_{\Re}\right)$. The relation between the complete map and an arbitrary algebraic map $m(\Re, \mathfrak{E}(A), \Upsilon)$ described in Theorem 29 now shows that $X$ covers $\mathcal{E}^{\prime}\left(\mathfrak{a}_{\mathfrak{R}}\right)$ if and only if $\Upsilon$ covers $\mathscr{E}^{\prime}(\mathfrak{a})$ in $\mathbb{E}(A)$ : for the univocal correspondence set up there between $\mathscr{E}^{\prime}\left(\mathfrak{a}_{\Re}\right)$ and $\mathfrak{E}^{\prime}(\mathfrak{a})$ carries $\mathfrak{X}(\mathfrak{r}) \mathfrak{E}^{\prime}\left(\mathfrak{a}_{\mathfrak{R}}\right)$ into $\mathfrak{V}(\mathfrak{r}) \mathfrak{E}^{\prime}(\mathfrak{a})$ and, conversely, carries $\mathfrak{V}(\mathfrak{r}) \mathfrak{E}^{\prime}(\mathfrak{a})$ back into $\mathfrak{X}(\mathfrak{r}) \mathbb{E}^{\prime}\left(a_{\mathfrak{r}}\right)$. The present theorem is thus completely proved.

The criterion for absolute closure with respect to $H$-extension which has just been established can now be replaced by a more familiar criterion, which we give in a somewhat generalized form.

TheOREM 50. A $T_{0}$-space $\Re$ is absolutely closed with respect to immediate or with respect to strict $H$-extension if and only if every covering family of open sets in $\Re$ contains a finite subfamily of open sets with closures which cover $\Re$. In this criterion, the sufficiency is maintained even if the covering families considered be restricted to be subfamilies of an arbitrary basis for $\Re$.

We establish this theorem by consideration of the map $m\left(\Re, \&\left(A_{\Re}\right), X\right)$, recalling that every open set in $\Re$ is a member of $A_{\Re}$. The open sets in any family $G$ which covers $\Re$ are represented in $\mathbb{E}\left(A_{\Re}\right)$ by sets $\&(a), a \epsilon A_{\Re}$, with the property that every set $\mathfrak{X}(\mathfrak{r})$ is contained in at least one of them. If $\mathcal{X}$ covers $\mathbb{F}^{\prime}\left(\mathfrak{a}_{\Re}\right)$, the family of sets $\mathbb{E}(a)$ also covers $\mathscr{E}^{\prime}\left(\mathfrak{a}_{\Re}\right)$. Thus the bicompactness of $\mathbb{E}^{\prime}\left(a_{\Re}\right)$ establishes the existence of open sets $a_{1}, \cdots, a_{n}$ in $G$ such that $\mathbb{F}^{\prime}\left(\mathfrak{a}_{\Re}\right) \subset \mathbb{E}\left(a_{1}\right) \cup \cdots \cup \mathfrak{E}\left(a_{n}\right)$. The relation $\mathfrak{X}(\mathfrak{r}) \mathbb{E}^{\prime}\left(\mathfrak{a}_{\mathfrak{R}}\right) \neq 0$ implies the relation $\mathfrak{X}(\mathfrak{r}) \mathfrak{E}\left(a_{k}\right) \neq 0$ for at least one index $k$ corresponding to the point $\mathfrak{r}$ in $\Re$. According to Theorem 28 , the relations $\mathfrak{X}(\mathfrak{r}) \mathfrak{E}\left(a_{k}\right) \neq 0$ and $\mathfrak{r} \in a_{\bar{k}}$ are equivalent. Hence we obtain the desired relation $a_{1}^{-} \vee \cdots \vee a_{n}^{-}=\Re, a_{1} \epsilon G, \cdots, a_{n} \in G$. On the other hand, if $\mathcal{X}$ does not cover $\mathbb{E}^{\prime}\left(\mathfrak{a}_{\Re}\right)$, let $\mathfrak{p}$ be a point of $\mathbb{E}^{\prime}\left(\mathfrak{a}_{\Re}\right)$ which belongs to no set $\mathfrak{X}$. Then if $\mathfrak{r}$ is any point of $\Re$, there exists an element $b(\mathfrak{r})$ in $A_{\Re}$ such that $\mathfrak{X}(\mathfrak{r}) \subset \mathbb{E}(b(\mathfrak{r})), p \in \mathbb{F}^{\prime}(b(\mathfrak{r}))$. If $a(\mathfrak{r})=b(\mathfrak{r})^{\prime-\prime}$ is the interior of $b(\mathfrak{r})$, the relation $a(\mathfrak{r}) \equiv b(\mathfrak{r})\left(\bmod a_{\Re}\right)$ shows that $\mathbb{E}(a(\mathfrak{r})) \mathfrak{E}^{\prime}\left(\mathfrak{a}_{\Re}\right)=\mathfrak{E}(b(\mathfrak{r})) \mathfrak{E}^{\prime}\left(a_{\Re}\right)$ and hence that $p \in \mathbb{E}^{\prime}(a(\mathfrak{r}))$; and the relation $\mathfrak{r} \in b(\mathfrak{r})^{\prime \prime}=a(\mathfrak{r})$ shows in accord- 
ance with Theorem 28 that $\mathfrak{X}(\mathfrak{r}) \subset \mathbb{E}(a(\mathfrak{r}))$. Thus the sets $a(\mathfrak{r}), \mathfrak{r} \in \Re$, constitute a family $G$ of open sets covering $\Re$. If $a_{1}, \cdots, a_{n}$ is any finite subfamily of $G$, we have $\mathfrak{p} \in \mathbb{E}^{\prime}\left(a_{1}\right) \cdots \mathbb{E}^{\prime}\left(a_{n}\right)=\mathbb{E}(a)$, where $a=a_{1}^{\prime} \cdots a_{n}^{\prime}$. Since $\{\mathfrak{p}\}$ is an $\mathcal{X}$-set in accordance with Theorem 34 , there exists a point $r$ such that $\mathfrak{X}(\mathfrak{r}) \subset \mathbb{E}(a)$. By virtue of Theorem 28, we conclude that $r \in a^{\prime \prime}=\left(a_{1} \vee \cdots \vee a_{n}\right)^{-\prime}$ $=\left(a_{1}^{-} \vee \cdots \vee a_{n}^{-}\right)^{\prime}$. Hence no finite subfamily of $G$ has the property that the closures of its members cover $\Re$. On comparing these results with those established in Theorem 49, we see that the first part of the present theorem is proved.

We still wish to show that, if the indicated property holds merely for covering families chosen from an arbitrary fixed basis in $\Re$, then $\Re$ is absolutely closed with respect to immediate or strict $H$-extension. We obtain this result by showing that we can pass from such restricted covering families to quite general families. Thus let $G$ be an arbitrary covering family of open sets. If $\mathfrak{r}$ is any point of $\Re$, there exists at least one set $\mathfrak{G}(\mathfrak{r})$ in $G$ which contains $\mathfrak{r}$; and hence there exists in the given fixed basis a set $\mathfrak{S}(\mathfrak{r})$ such that $\mathfrak{r} \in \mathfrak{W}(\mathfrak{r}) \subset \mathfrak{S}(\mathfrak{r})$. The family $\mathfrak{F C}$ of all sets $\mathfrak{S}(\mathfrak{r})$ is then a subfamily of the given basis which covers $\Re$. By hypothesis, therefore, there exist sets $\mathfrak{W}_{1}, \cdots, \mathfrak{S}_{n}$ in $\mathfrak{H C}$ such that $\mathfrak{S}_{1}^{-} \cup \cdots \cup \mathfrak{S}_{n}^{-}=\Re$. The corresponding sets $\mathfrak{S}_{1}, \cdots, \mathfrak{S}_{n}$ in $G$ then have the property $\mathscr{G}_{1}^{-} \cup \cdots \cup \mathfrak{S}_{n}^{-} \supset \mathfrak{S}_{1}^{-} \mathbf{u} \cdots \cup \mathfrak{E}_{n}^{-}=\Re$. This completes the proof.

Having examined the extension problem in a general way, we shall now proceed to consider specific imbedding and extension theorems, some of which, long formulated, have hitherto remained unproved. Our first result does not concern immediate extensions, but is conveniently stated at this point.

THEOREM 51. Let $\mathfrak{Q}_{\mathfrak{c}}$ be the $T_{0}$-space obtained by topologizing the family $Z$ of all closed sets in the Boolean space $\mathfrak{B}_{\mathrm{c}}$ in the usual way, $\mathrm{c}$ being any infinite cardinal number. Then $\mathfrak{Q}_{c}$ is a universal $T_{0}$-space of character $c$; in other words, every $T_{0}$-space $\Re$ of character not exceeding $\mathrm{c}$ is topologically equivalent to a subspace of $\mathfrak{Q}_{\mathfrak{c}}$.

From Theorem 23, we know that $\mathfrak{Q}_{\mathrm{c}}$ is a $T_{0}$-space of character not exceeding that of $\mathfrak{B}_{\mathfrak{c}}$. On the other hand, $\mathfrak{Q}_{\mathfrak{c}}$ contains $\mathfrak{B}_{\mathfrak{c}}$ as a subspace, since the one-element subsets of $\mathfrak{B}_{c}$ are members of $Z$; and its character is therefore not less than that of $\mathfrak{B}_{\mathfrak{c}}$. Hence the character of $\mathfrak{Q}_{\mathfrak{c}}$, like that of $\mathfrak{B}_{\mathfrak{c}}$, is equal to $c$.

Now if $\Re$ is an arbitrary $T_{0}$-space of character not exceeding $c$, it has a basis of cardinal number not exceeding $\mathrm{c}$; and the basic ring $A$ generated by such a basis has cardinal number not exceeding $c$. We consider the 
map $m(\Re, \&(A), X)$. Since the character of $\mathfrak{E}(A)$ does not exceed $\mathfrak{c}$, we may regard it as a closed subset of the Boolean space $\mathfrak{B}_{\mathrm{c}}$ in accordance with the results of Chapter $\mathrm{I}, \S 3$. Thereby we obtain a map $m\left(\Re, \mathfrak{B}_{\mathfrak{c}}, \Upsilon\right)$ where $\Upsilon$ is the subfamily of $Z$ consisting of the sets of $X$, now regarded as closed subsets of $\mathfrak{B}_{\mathfrak{c}}$. It follows immediately that $Z$ is an extension of $\Upsilon, \mathfrak{Q}_{\mathfrak{c}}$ of $\Re$, in accordance with Definition 13.

The discussion of universal finite $T_{0}$-spaces is omitted here. Some remarks on this subject will be found in the following section.

THEOREM 52. Every $T_{0}$-space $\Re$ has a strict $H$-extension $\mathfrak{Q}$ which has the same character as $\Re$ and which is absolutely closed with respect to immediate or with respect to strict $H$-extension. The space $\Re^{\prime}$ of points adjoined to $\Re$ in this extension may be taken as a totally-disconnected $H$-space.*

As in the preceding theorem, we consider a map $m(\Re, \&(A), X)$ determined by a basic ring $A$ generated by a basis for $\Re$ with minimal cardinal number $c$. The character of $\mathbb{E}(A)$ is then equal to $\mathfrak{c}$, if we exclude the trivial case of finite spaces $\Re$. We obtain the desired space $\mathscr{Q}$ by adjoining to the family $X$ all the one-element sets $\{\mathfrak{p}\}$ where $\mathfrak{p}$ is a point of ' $\mathcal{G}^{\prime}(\mathfrak{a})$ which belongs to no set $\mathfrak{X}$. It is then evident that $\mathfrak{Q}$ is an immediate $H$-extension of $\Re$ in accordance with Theorems 38 and 41 . The character of $\mathfrak{Q}$ is not less than that of its subspace $c$; and is not greater than $c$ because of Theorem 23. Hence the character of $\mathfrak{Q}$, like that of $\Re$, is equal to $c$. Moreover, the topology of the set of points adjoined to $\Re$ in the construction of $\mathfrak{Q}$ is equivalent to that of a subset of $\mathbb{F}^{\prime}(\mathfrak{a})$; it is therefore a totally-disconnected $H$-space.

We prove next that $\mathfrak{Q}$ is actually a strict extension of $\Re$. Let us consider an open set specified in $\mathfrak{Q}$ by the relations $\mathfrak{Z}(\mathfrak{q}) \subset \mathfrak{F}(a), a \in A, a \notin \mathfrak{a}$, where $\mathfrak{Z}(\mathfrak{q})$ is either a set in $X$ or one of the adjoined sets $\{\mathfrak{p}$. To this set we adjoin an arbitrary subset of $\Re^{\prime}$, thus obtaining a set $\mathfrak{F}$ in $\mathfrak{Q}$ and a corresponding family of sets $\mathfrak{Z}(\mathfrak{q}), \mathfrak{q} \in \mathfrak{F}$. We determine $\mathfrak{F}^{\prime-\prime}$, the interior of $\mathfrak{F}$. The sets $\mathfrak{Z}(\mathfrak{q})$ corresponding to $\mathfrak{F}^{\prime}$ are all the sets $\mathfrak{Z}(\mathfrak{q})$ such that $\mathfrak{Z}(\mathfrak{q}) \mathfrak{E}^{\prime}(a) \neq 0$, with the exception of the one-element sets which were involved in the adjunction described above. The sets $3(\mathfrak{q})$ corresponding to $\mathfrak{F}^{\prime}$ are precisely the sets $3(\mathfrak{q})$ such that $\mathfrak{Z}(\mathfrak{q}) \mathscr{E}^{\prime}(a) \neq 0$, as we see by examining the situation in detail. First, if $\mathfrak{Z}(\mathfrak{q}) \subset \mathbb{E}(a)$, the point $\mathfrak{q}$ cannot belong to $\mathfrak{F}^{\prime}$ since the assumed relation means that the open set specified in $\mathfrak{Q}$ by $\mathbb{Z} \subset \mathbb{E}(a)$ contains $q$ but does not contain any point of $\mathfrak{F}^{\prime}$. Secondly, if $\mathfrak{Z}(\mathfrak{q})=\{\mathfrak{p}\}$ is in $\mathfrak{F}^{\prime}(a)$, then the fact

* The problem of the existence of an immediate $H$-extension of an $H$-space $\Re$ under the requirements that the extension shall have the same character as $\Re$ and shall be absolutely closed with respect to immediate $H$-extension, was proposed by Alexandroff and Urysohn, Memoire sur les espaces topologiques compacts, Verhandelingen der Koninklijke Akademie van Wetenschappen te Amsterdam, Deel XIV, No. 1 (1929), p. 52. 
that $\{\mathfrak{p}\}$ is an $\mathcal{X}$-set shows that, whenever $\mathfrak{E}(b)$ contains $\mathfrak{p}$, then $\&\left(a^{\prime} b\right)$ $=\mathbb{F}^{\prime}(a) \mathfrak{E}(b)$ contains $\{\mathfrak{p}\}$ and also a set $\mathfrak{X}(\mathfrak{r}), \mathfrak{r} \in \Re$; since the point $\mathfrak{r}$ thus belongs to $\mathfrak{F}^{\prime}$ in accordance with the fact that $\mathfrak{X}(\mathfrak{r}) \subset \mathbb{F}^{\prime}(a)$, we conclude that $q$ belongs to $\mathfrak{F}^{\prime}$. In view of this characterization of the set $\mathfrak{F}^{\prime}$, we see that $\mathfrak{F}^{\prime \prime}$ consists of those points $\mathfrak{q}$ for which $\mathbb{Z}(\mathfrak{q}) \subset \mathbb{E}(a)$. Now let $\mathbb{B}$ be any non-void open set in $\mathfrak{Q}$ and let $q$ be any point of $\mathfrak{S}$. Since the sets $\mathbb{S}(a)$ specified in $\mathfrak{Q}$ by the relations $\mathfrak{Z}(\mathfrak{q}) \subset \mathbb{E}(a), a \epsilon A$, constitute a basis in $\mathfrak{Q}$, we can find an element $a$ in $A$ and such a corresponding set $\mathbb{S}(a)$ in $\mathfrak{Q}$ that $q \in(S)(a) \subset \mathbb{B}$. Obviously, the relation $\mathfrak{Z}(\mathfrak{q}) \subset \mathbb{E}(a)$ shows that $a \notin \mathfrak{a}$. Then, if $\mathfrak{S}$ is any subset of $\mathfrak{R}^{\prime}$, we have $\mathfrak{H}(a) \Delta \mathfrak{S} \subset \mathfrak{F},(\mathfrak{S}(a) \Delta \mathfrak{S})^{\prime-^{\prime}} \subset \mathfrak{F}^{\prime{ }^{\prime}}$, where $\mathfrak{F}=\mathfrak{S}(a) \cup \mathfrak{S}$. As we showed above, $\mathfrak{F}^{\prime-^{\prime}}=\mathfrak{S}(a)$. Hence we see that $(\mathfrak{S}(a) \Delta \mathfrak{S})^{\prime-\prime} \subset \mathfrak{S}$. By reference to Definition 14 , we conclude that $\mathfrak{Q}$ is a strict extension of $\Re$.

Finally, we prove that $\mathfrak{Q}$ is closed with respect to immediate or strict $H$-extension, appealing for this purpose to the criterion stated in Theorem 50 . If a family of the basis sets $\mathbb{S}(a)$, specified by $\mathfrak{Z}(\mathfrak{q}) \subset \mathbb{E}(a)$, covers $\mathfrak{Q}$, then the corresponding sets $\mathbb{E}(a)$ contain every set $\mathfrak{Z}(\mathfrak{q})$ and therefore cover $\mathfrak{F}^{\prime}(\mathfrak{a})$. Hence there exist elements $a_{1}, \cdots, a_{n}$ associated with the given covering family and having the property $\xi^{\prime}(\mathfrak{a}) \subset \mathbb{E}\left(a_{1}\right) \cup \cdots \cup \mathbb{\cup}\left(a_{n}\right)$. Now we can show that $\mathfrak{Z}(\mathfrak{q}) \mathfrak{E}^{\prime}(\mathfrak{a}) \mathfrak{E}(a) \neq 0$ implies $\mathfrak{q} \in(\mathfrak{S}-(a)$. In order to do so it is sufficient to prove that $\mathcal{Z}(\mathfrak{q}) \mathbb{E}^{\prime}(\mathfrak{a}) \mathbb{E}(a) \neq 0$ and $q \in \mathbb{S}(b)$ imply $\mathbb{S}(a) \mathfrak{S}(b) \neq 0$. From the assumed relations $\mathbb{Z}(\mathfrak{q}) \mathbb{F}^{\prime}(\mathfrak{a}) \mathbb{E}(a) \neq 0, \mathfrak{Z}(\mathfrak{q}) \subset \mathbb{E}(b)$, we obtain the relations $\mathfrak{E}(a b) \mathbb{E}^{\prime}(\mathfrak{a})=\mathbb{E}(b) \mathbb{E}^{\prime}(\mathfrak{a}) \mathbb{E}(a) \supset \mathfrak{Z}(\mathfrak{q}) \mathbb{E}^{\prime}(\mathfrak{a}) \mathbb{E}(a) \neq 0$ and thus conclude that $a b \notin \mathfrak{a}$. Hence the interior of $a b$ relative to $\Re$ contains at least one point $\mathfrak{r}$, and $\mathfrak{X}(\mathfrak{r}) \subset \mathbb{E}(a b), \mathfrak{X}(\mathfrak{r}) \subset \mathbb{E}(a), \mathfrak{X}(\mathfrak{r}) \subset \mathbb{E}(b), \mathfrak{r} \in \mathfrak{S}(a) \mathfrak{B}(b)$. If now $\mathfrak{q}$ is any point in $\mathfrak{Q}$, we have $\mathfrak{B}(\mathfrak{q}) \mathbb{F}^{\prime}(\mathfrak{a}) \mathfrak{F}\left(a_{1}\right)$ u $\ldots$ u $\mathfrak{Z}(\mathfrak{q}) \mathbb{F}^{\prime}(\mathfrak{a}) \mathfrak{F}\left(a_{n}\right)=\mathfrak{Z}(\mathfrak{q}) \mathbb{F}^{\prime}(\mathfrak{a})\left[\mathfrak{F}\left(a_{1}\right) \mathbf{u} \ldots\right.$ u $\left.\mathbb{E}\left(a_{n}\right)\right]=Z(\mathfrak{q}) \mathfrak{F}^{\prime}(\mathfrak{a}) \neq 0$, so that $\mathbb{Z}(\mathfrak{q}) \mathfrak{F}^{\prime}(\mathfrak{a}) \mathfrak{F}\left(a_{k}\right) \neq 0, \mathfrak{q} \in\left(\mathfrak{S}-\left(a_{k}\right)\right.$ for some index $k$. Thus we find that $\mathfrak{B}-\left(a_{1}\right) \cup \cdots \cup\left(\mathfrak{S}-\left(a_{n}\right)=\mathfrak{Q}\right.$. By the criterion of Theorem 50 , it results that $\mathfrak{Q}$ is absolutely closed with respect to immediate or strict $H$-extension.

Finally we shall establish an important characterization of the bicompact $H$-spaces.

THEOREM 53. In order that an $H$-space $\Re$ be bicompact, it is necessary and sufficient that every closed subset of $\Re$ be absolutely closed with respect to immediate or with respect to strict $H$-extension.*

The necessity of the condition is trivial: every closed subset of a bicompact space is bicompact and thus satisfies the criterion given in Theorem 50.

* This theorem was proposed by Alexandroff and Urysohn, Mêmoire sur les espaces topologiques compacts, Verhandelingen der Koninklijke Akademie van Wetenschappen te Amsterdam, Deel XIV, No. 1 (1929), p. 50; but was proved only in case $\Re$ is separable. 
The sufficiency of the condition is more difficult to establish. In our demonstration we appeal to the following criterion for bicompactness: in order that an $H$-space be bicompact, it is necessary and sufficient that every transfinite descending sequence of non-void closed subsets have a non-void intersection.* In the given space $\Re$, let $\left\{\mathfrak{F}_{\alpha}\right\}$ be such a sequence, defined for all ordinals $\alpha$ preceding a given ordinal $\omega$ : we have $\mathfrak{F}_{\alpha} \neq 0, \mathfrak{F}_{\alpha}^{-}=\mathfrak{F}_{\alpha}$, and $\mathfrak{F}_{\alpha} \supset \mathfrak{F}_{\beta}$ for $\alpha<\beta$. We consider the complete algebraic map $m\left(\Re, \&\left(A_{\Re}\right), \mathcal{X}\right)$ and the subfamilies $X_{\alpha}$ of $X$ specified by the relation $\mathfrak{r} \in \mathfrak{F}_{\alpha}$. If $\mathbb{F}_{\alpha}\left(A_{\Re}\right)$ is the closure of the union of the sets $\mathfrak{X}(\mathfrak{r})$ in $X_{\alpha}$, the map $m\left(\mathfrak{F}_{\alpha}, \mathfrak{F}_{\alpha}\left(A_{\Re}\right), X_{\alpha}\right)$ then has the properties described in Theorems 36 and 37. In particular the union $\mathfrak{F}\left(X_{\alpha}\right)$ of the minimal $X_{\alpha}$-sets is closed and bicompact in $\mathbb{E}\left(A_{\Re}\right)$. If $\alpha_{1}, \cdots, \alpha_{n}$ are ordinals preceding $\omega$ such that $\alpha_{k}<\alpha_{k+1}$ for $k=1, \cdots, n-1$, then the relation $\mathfrak{F}_{\alpha_{k}} \supset \mathfrak{F}_{\alpha_{k+1}}$ implies $X_{\alpha_{k}} \supset X_{\alpha_{k+1}}$. Hence each minimal $X_{\alpha_{k+1}}$ set is an $X_{\alpha_{k}}$-set and contains a minimal $X_{\alpha_{k}}$-set, for $k=1, \cdots, n$. Hence we see that $\mathfrak{F}\left(X_{\alpha_{1}}\right) \cdots \mathfrak{F}\left(X_{\alpha_{n}}\right) \neq 0$. Since no finite intersection of the closed sets $\mathfrak{F}\left(X_{\alpha}\right)$, $\alpha<\omega$, is void, we conclude that there exists at least one point $p$ common to them all. By combining results of Theorems $28,34,36,37$ and 49, we can now show that this point $p$ determines a point $\mathfrak{r}$ in $\Re$ such that $\mathfrak{r} \epsilon \mathfrak{F}_{\alpha}$ for $\alpha<\omega$. Theorems 36 and 37 permit us to correlate the map $m\left(\mathfrak{F}_{\alpha}, \mathfrak{F}_{\alpha}\left(A_{\Re}\right), X_{\alpha}\right)$ with an algebraic map $m\left(\mathfrak{F}_{\alpha}, \mathfrak{B}_{\alpha}, \Upsilon_{\alpha}\right)$ in such a way that the $\Upsilon_{\alpha}$-sets are put in correspondence with the $X_{\alpha}$-sets, inclusion relations being preserved, the sets in $\Upsilon_{\alpha}$ corresponding biunivocally with the sets in $X_{\alpha}$, and the minimal $\Upsilon_{\alpha}$-sets corresponding biunivocally with the minimal $X_{\alpha}$-sets. By Theorem 28 , our hypothesis that $\Re$ is an $H$-space implies that the distinct sets in $X_{\alpha}$ and also those in $\Upsilon_{\alpha}$ are disjoint. By Theorems 34 and 49, our hypothesis that $\mathfrak{F}_{\alpha}$ is absolutely closed with respect to immediate or strict extension implies that each minimal $\Upsilon_{\alpha}$-set is contained in at least one set belonging to $\Upsilon_{\alpha}$. Consequently, we see that each minimal $X_{\alpha}$-set is contained in exactly one set belonging to $X_{\alpha}$. Thus the relation $p \in \mathfrak{F}\left(X_{\alpha}\right)$ implies the existence of a minimal $X_{\alpha}$-set $\mathfrak{Z}_{\alpha}$ such that $p \in \mathfrak{Z}_{\alpha}$. This minimal $X_{\alpha}$-set is contained in a set $\mathfrak{X}(\mathfrak{r})$ where $\mathfrak{X}(\mathfrak{r}) \in \mathcal{X}_{\alpha}$ or, equivalently, $\mathfrak{r} \in \mathfrak{F}_{\alpha}$. Since the sets $\mathfrak{X}$ are disjoint, the relation $\mathfrak{p} \in \mathfrak{X}(\mathfrak{r})$ implies that $\mathfrak{r}$ is independent of $\alpha$ for all $\alpha<\omega$. We conclude that $\mathfrak{r}$ is common to the sets $\mathfrak{F}_{\alpha}, \alpha<\omega$. It follows, in accordance with the criterion cited above, that $\Re$ is a bicompact space. The proof of the theorem is thus complete.

5. Totally-disconnected and discrete spaces. In concluding the present

* See Alexandroff and Urysohn, Mathematische Annalen, vol. 92 (1924), pp. 258-266; especially pp. 259-261; or Alexandroff and Urysohn, Mémoire sur les espaces topologiques compacts, Verhandelingen der Koninklijke Akademie van Wetenschappen te Amsterdam, Deel XIV, No. 1 (1929), pp. $8-12$. 
chapter, we shall discuss briefly two special kinds of space: totally-disconnected spaces, as defined in Chapter I, $\$ 1$, and discrete spaces, as defined below. We first have the following theorem.

Theorem 54. A $T_{0}$-space $\Re$ is totally-disconnected if and only if it has a biunivocal continuous image $\subseteq$ which is a subspace of a Boolean space.

We begin by proving the sufficiency of the stated condition. Let $f$ be a biunivocal continuous correspondence from $\Re$ to $\subseteq$ where $\subseteq$ is a subspace of a Boolean space $\mathfrak{B}$. Let $\mathfrak{r}_{1}$ and $\mathfrak{r}_{2}$ be distinct points in $\Re$, let $\varepsilon_{1}=f\left(\mathfrak{r}_{1}\right)$ and $\varepsilon_{2}=f\left(\mathfrak{r}_{2}\right)$ be their correspondents in $\mathfrak{S}$. By hypothesis $\varepsilon_{1} \neq z_{2}$, so that there exist closed-and-open sets $\mathfrak{F}_{1}$ and $\mathfrak{F}_{2}$ in $\mathfrak{B}$ with the properties $\mathbb{8}_{1} \in \mathfrak{F}_{1}, \mathbb{Z}_{2} \in \mathfrak{F}_{2}$, $\mathfrak{F}_{1} \mathfrak{F}_{2}=0, \mathfrak{F}_{1} \cup \mathfrak{F}_{2}=\mathscr{B}$. The sets $\mathfrak{F}_{1} \subseteq$ and $\mathfrak{F}_{2} \subseteq$ are closed-and-open relative to $\mathfrak{S}$, so that their antecedents $\mathfrak{S}_{1}=f^{-1}\left(\mathfrak{F}_{1} \subseteq\right), \mathfrak{W}_{2}=f^{-1}\left(\mathfrak{F}_{2} \subseteq\right)$ in $\Re$ are closed-andopen. Since $\mathfrak{r}_{1} \in \mathfrak{S}_{1}, \mathfrak{r}_{2} \in \mathfrak{S}_{2}, \mathfrak{S}_{1} \mathfrak{W}_{2}=0$, and $\mathfrak{S}_{1} \cup \mathfrak{S}_{2}=\Re$, we conclude that $\Re$ is totally-disconnected.

We pass now to the necessity of the indicated condition. If $\Re$ is totallydisconnected, the subsets of $\Re$ which are both open and closed constitute a Boolean ring $A$ : for, if $a$ and $b$ are both open and closed, so also are $a b, a \vee b$, $a^{\prime}, b^{\prime}$, and $a+b=a b^{\prime} \vee a^{\prime} b$. The ring $A$ must further be a reduced algebra of classes in $\Re$ in accordance with $\mathrm{R}$ Definition 10 , since, whenever $\mathfrak{r}_{1}$ and $\mathfrak{r}_{2}$ are distinct points in $\Re$, there exists an element $a$ in $A$ such that $\mathfrak{r}_{1} \epsilon a, \mathfrak{r}_{2} \epsilon a^{\prime}$. Hence the subclass of $A$ specified by $\mathfrak{r} a$ is a prime ideal $\mathfrak{p}(\mathfrak{r})$ in accordance with $\mathrm{R}$ Theorem 58. The non-void sets in $A$ can now be used to define a new neighborhood topology over the class $\Re$ : to each point of $\Re$ we assign as neighborhoods the members of $A$ which contain it. The resulting topological space, we designate as $\mathfrak{S}$. It is evident that the identical correspondence $\mathfrak{r} \rightarrow \mathfrak{r}$ carries $\Re$ into $\subseteq$ biunivocally and continuously. Thus, to complete our proof, we have to show that $\subseteq$ is topologically equivalent to a subspace of a Boolean space. The Boolean ring $A$ is evidently a basic ring for $\mathfrak{S}$. If we construct the algebraic map $m(\mathfrak{S}, \mathfrak{E}(A), X)$, we find that the set $\mathfrak{X}(\mathfrak{r})$ corresponding to a point $\mathfrak{r}$ of $\mathfrak{S}$ consists of a single point in $\mathbb{E}(A)$, namely, the point $\mathfrak{p}(\mathfrak{r})$ defined above as a prime ideal. For, whenever $a$ is $A$, we have $a=a^{-}=a^{\prime-\prime}$ so that $\mathfrak{X}(\mathfrak{r}) \subset \mathbb{E}(a)$ is equivalent to $\mathfrak{r} \in a$ in accordance with Theorem 28. Thus $\subseteq$ is topologically equivalent to the subspace of $\mathbb{E}(A)$ consisting of the points $\mathfrak{p}(\mathfrak{r}), \mathfrak{r} \in \mathfrak{S}$.

We may complete the preceding theorem by the following result.

THeOREM 55. A $T_{1}$-space $\Re$ is topologically equivalent to a subspace of a Boolean space if and only if, whenever $\mathfrak{F}$ is a closed set in $\Re$ and $\mathfrak{r}$ is a point in $\mathfrak{F}^{\prime}$, there exist closed sets $\mathfrak{F}_{1}$ and $\mathfrak{F}_{2}$ in $\Re$ such that $\mathfrak{F} \subset \mathfrak{F}_{1}, \mathfrak{r} \in \mathfrak{F}_{2}, \mathfrak{F}_{1} \mathfrak{F}_{2}=0$ and $\mathfrak{F}_{1} \cup \mathfrak{F}_{2}=\Re$. 
If $\Re$ is a subspace of a Boolean space $\mathscr{B}=\mathfrak{F}(A)$, then $\mathfrak{r} \in \mathfrak{F}^{\prime}$, where $\mathfrak{F}$ is closed in $\Re$, implies the existence of a set $\mathscr{E}(a) \Re$, where $a$ is in the Boolean ring $A$, which is open relative to $\Re$ and which has the property that $\mathfrak{F} \mathfrak{E}(a) \Re=0$. Hence we can take $\mathfrak{F}_{1}=\mathfrak{F}^{\prime}(a) \Re, \mathfrak{F}_{2}=\mathfrak{F}(a) \Re$.

If $\Re$ has the property indicated in the theorem, the closed-and-open subsets of $\Re$ constitute a basis in $\Re$ : for if $\mathfrak{r}$ is any point of $\Re$ and $(S)$ any open set containing $\mathfrak{r}$, the application of this property to $\mathfrak{r}$ and the closed set $\mathfrak{F}=\mathfrak{G}^{\prime}$ yields a closed-and-open set $\mathfrak{F}_{2}$ such that $\mathfrak{r} \in \mathfrak{F}_{2} \subset \mathfrak{S}=\mathfrak{F}^{\prime}$. The assumption that $\Re$ is a $T_{1}$-space implies that any one-element subset of $\Re$ is closed and hence that $\Re$ is totally-disconnected. Accordingly, the associated space $\subseteq$ constructed from $\Re$ in the manner described in the proof of Theorem 54 is topologically equivalent to $\Re$ and the ring $A$ is a basic ring for $\Re$. We conclude that $\Re$ is equivalent to a subspace of $\mathbb{E}(A)$.

We pass now to the consideration of discrete spaces, defined as follows:

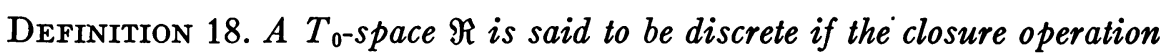
has the property

$$
\left(\sum_{\mathfrak{R e C A}} \mathfrak{A}\right)^{-}=\sum_{\mathfrak{A} \in \mathcal{A}} \mathfrak{A}^{-}
$$

for any family $\mathcal{A}$ of subsets $\mathfrak{A}$ of $\Re$.

The topology of discrete spaces is described in the following statement, which we give without proof.

TheOREM 56. The following properties of a $T_{0}$-space are equivalent:

(1) $\Re$ is a discrete space;

(2) every union of closed sets is closed;

(3) every intersection of open sets is open.

If the intersection of all open sets containing a fixed point $\mathfrak{r}$ in a discrete $T_{0^{-}}$-space be denoted by $\mathfrak{S}(\mathfrak{r})$, then

(4) the sets $\mathfrak{S}(\mathfrak{r}), \mathfrak{r} \in \Re$, constitute a minimal basis for $\Re$-that is, a basis contained in every basis for $\Re$;

(5) $\mathbb{S}\left(\mathfrak{r}_{1}\right)=\mathfrak{S}\left(\mathfrak{r}_{2}\right)$ implies $\mathfrak{r}_{1}=\mathfrak{r}_{2}$;

(6) if $\mathfrak{S}\left(\mathfrak{r}_{1}\right) \mathfrak{S}\left(\mathfrak{r}_{2}\right) \neq 0$, then $\mathfrak{r}_{3} \in \mathfrak{S}\left(\mathfrak{r}_{1}\right) \mathfrak{S}\left(\mathfrak{r}_{2}\right)$ implies $\mathfrak{S}\left(\mathfrak{r}_{3}\right) \subset \mathfrak{S}\left(\mathfrak{r}_{1}\right) \mathfrak{S}\left(\mathfrak{r}_{2}\right)$.

The relation $\mathfrak{r}_{1}<\mathfrak{r}_{2}$ defined by $\mathfrak{r}_{1} \neq \mathfrak{r}_{2}, \mathbb{S}\left(\mathfrak{r}_{1}\right) \subset \mathbb{S}\left(\mathfrak{r}_{2}\right)$ has the properties of a partial order in $\Re$. In terms of this relation, the closure of an arbitrary subset $\mathfrak{A}$ of $\Re$ is the set $\mathfrak{A}-$ consisting of all points $\mathfrak{r}$ such that $\mathfrak{r} \geqq \mathfrak{r}_{0}$ for some $\mathfrak{r}_{0}$ in $\mathfrak{A}$. Every finite $T_{0}$-space is discrete.

We may give a converse of part of this theorem, also without detailed proof.

* See Alexandroff, Comptes Rendus (Paris), vol. 200 (1935), pp. 1649-1651. 
THEOREM 57. If $\Re$ is any partially-ordered class, the introduction of the closure operation defined by

$$
\mathfrak{A}^{-}=\sum_{\mathfrak{r} \mathbb{v e}_{\mathfrak{A}}} \sum_{\mathfrak{r} \geq \mathfrak{r}_{0}}\{\mathfrak{r}\}
$$

converts $\Re$ into a discrete $T_{0}$-space; in this space the ordering relation provided by Theorem 56 coincides with the one given originally in $\Re .^{*}$

With the aid of the mapping theory, we can now represent a discrete space in terms of Boolean spaces or, equivalently, a partially ordered set in terms of Boolean rings.

THEOREM 58. Let $A$ be the topological ring for a discrete $T_{0}$-space generated by the basis of all sets $\mathbb{S}(\mathfrak{r})$, described in Theorem 56 . In the map $m(\Re, \mathfrak{F}(A), X)$, the relation $\mathfrak{X}(\mathfrak{r})=\mathfrak{E}(\mathfrak{S}(\mathfrak{r}))$ is valid. Hence the points $\mathfrak{r}$ of $\Re$ and the ordering relation between them are represented by elements of the ring $A$ and the relation of proper inclusion between them. Conversely, any subclass of a Boolean ring $A$ can be regarded as a discrete $T_{0}$-space by virtue of the fact that it is partially ordered in terms of the relation of proper inclusion defined in $A$.

The theorem is obvious, once we prove that $\mathfrak{X}(\mathfrak{r})=\mathfrak{E}(\mathfrak{S}(\mathfrak{r}))$. Since $\mathfrak{r}$ is interior to $\mathfrak{S}(\mathfrak{r})$, we have $\mathfrak{X}(\mathfrak{r}) \subset \mathfrak{E}(\mathfrak{S}(\mathfrak{r}))$ by virtue of Theorem 28 . On the other hand, if $\mathbb{E}(a) \supset \mathfrak{X}(\mathfrak{r})$ for $a \epsilon A$, we have $\mathfrak{r} \epsilon a^{\prime-\prime}$ in accordance with Theorem 28; and we conclude that $\mathfrak{S}(\mathfrak{r}) \subset a^{\prime{ }^{\prime}} \subset a, \mathfrak{X}(\mathfrak{r}) \subset \mathbb{E}(\mathfrak{S}(\mathfrak{r})) \subset \mathbb{E}(a)$. Hence we must have $\mathfrak{X}(\mathfrak{r})=\mathfrak{E}(\mathfrak{S}(\mathfrak{r}))$ in accordance with Theorem 26 , as we wished to prove. We may note that, by virtue of Theorem 56 (6) the relation $\mathfrak{X}\left(\mathfrak{r}_{1}\right) \mathfrak{X}\left(\mathfrak{r}_{2}\right) \neq 0$ implies the existence of a point $\mathfrak{r}_{3}$ such that $\mathfrak{X}\left(\mathfrak{r}_{3}\right) \subset \mathfrak{X}\left(\mathfrak{r}_{1}\right) \mathfrak{X}\left(\mathfrak{r}_{2}\right)$ : for $\mathbb{E}\left(\mathfrak{S}\left(\mathfrak{r}_{1}\right) \mathfrak{S}\left(\mathfrak{r}_{2}\right)\right)=\mathfrak{E}\left(\mathfrak{S}\left(\mathfrak{r}_{1}\right)\right) \mathfrak{E}\left(\mathfrak{S}\left(\mathfrak{r}_{2}\right)\right)=\mathfrak{X}\left(\mathfrak{r}_{1}\right) \mathfrak{X}\left(\mathfrak{r}_{2}\right) \neq 0$ implies $\mathbb{S}\left(\mathfrak{r}_{1}\right) \mathfrak{S}\left(\mathfrak{r}_{2}\right) \neq 0$. The interpretation of this theorem which states that any partially ordered set can be imbedded in a Boolean ring with unit has been established abstractly by MacNeille. $\dagger$

From Theorem 58, we obtain the following special result.

THEOREM 59. Let $\mathfrak{Q}_{n}$ be the finite $T_{0}$-space obtained by the application of Theorem 57 to the partially-ordered class of all non-zero elements in a finite Boolean ring of $2^{n+1}$ elements, $n=0,1,2, \ldots$. Then $\mathfrak{Q}_{n}$ is an abstract $n$-simplex, under a suitable topology. The spaces $\mathfrak{Q}_{n}$ are universal spaces for all finite $T_{0}$-spaces; in other words, a finite $T_{0}$-space $\Re$ is topologically equivalent to a subspace of $\mathfrak{Q}_{n}$ for sufficiently great $n$.

* See Alexandroff, Comptes Rendus (Paris), vol. 200 (1935), pp. 1649-1651.

$\dagger$ H. M. MacNeille, The theory of partially ordered sets, Harvard doctoral dissertation (1935), not yet published. A summary is given in Proceedings of the National Academy of Sciences, vol. 22 (1936), pp. 45-50. 
We can exclude the trivial case where $\Re$ is void. If $\Re$ is a finite non-void $T_{0}$-space, it is discrete and can be studied by means of Theorem 58 . The ring $A$ evidently is finite, the number of its elements being $2^{n+1}, n \geqq 0$; and the Boolean space $\&(A)$ consists of $n+1$ isolated points. The family $Z$ of all sets $\&(a)$, where $a \in A$ and $a \neq 0$, evidently consists of all non-void subsets of $\mathbb{E}(A)$. If $Z$ is topologized in the usual way, the resulting $T_{0}$-space is equivalent to the space $\mathfrak{Q}_{n}$ : for it is evidently equivalent to the space obtained by regarding the non-zero elements of the ring $A$ as a partially ordered set and introducing the topology described in Theorem 57. In the map $m(\Re, \&(A), X)$, the family $X$ is contained in $Z$. Hence $\Re$ is topologically equivalent to a subspace of $\mathfrak{Q}_{n}$, as we wished to prove.

As we have just seen, the space $\mathfrak{Q}_{n}$ is equivalent_to the $T_{0}$-space constructed by consideration of all the non-void subsets of a Boolean space consisting of $n+1$ isolated points. If we term these subsets "cells," referring to a subset with $k+1$ elements, $k \geqq 0$, in particular as a " $k$-cell," we can easily make the desired connection with an $n$-simplex. If one cell is contained in a second, we call it an "edge" of the latter. It is now clear that, as our terminology suggests, the "cells" and the relation of being an "edge" can be exactly represented by the $k$-dimensional "edges" of an $n$-dimensional Euclidean cell, for $k=0,1, \cdots, n$, and the relation of inclusion between them. The topology of the abstract "cell" space, as we have already seen, can be described by the statement that the closure of any given set of "cells" is the class of all "cells" having at least one of the given "cells" as an "edge." Thus we may regard the $T_{0}$-space $\mathfrak{Q}_{n}$ as an abstract $n$-simplex, the topology of the latter being described in the manner just indicated.

By dualization, it is possible to pass to the more familiar abstract $n$-simplex in which the closure of any given set of "cells" is the class of all "cells" which are "edges" of at least one of the given "cells." We shall not consider this question further.*

Theorem 59 shows that the finite $T_{0}$-spaces are the subspaces of abstract $n$-simplexes, that is, are abstract complexes. In addition, it completes the results obtained in Theorem 51 for infinite $T_{0}$-spaces. From the present point of view, therefore, the spaces $\mathfrak{Q}_{\mathfrak{c}}$ may be regarded as abstract simplexes of infinite dimensionality.

\section{CHAPTER III. STRONGER SEPARATION CONDITIONS}

1. Semi-regular spaces. In the general theory of Boolean maps as developed in Chapter II, we have considered $T_{0^{-}}, T_{1^{-}}$, and $H$-spaces on an equal

\footnotetext{
${ }^{*}$ For further analysis, see AH, pp. 132 133 .
} 
footing. Our results show that the imposition of the $T_{1^{-}}$or the $H$-separation property yields no essential simplification of the theory; in other words, the Boolean maps of $T_{1}$-spaces and $H$-spaces are quite as complicated as those of $T_{0}$-spaces. The search for types of space which may admit simplification of the general mapping theory directs our attention to the various stronger separation properties, such as regularity and normality. Upon examination, we discover that the general theory is already in such a form as to suggest the procedure for its own simplification: it is obvious that one should examine first of all the conditions under which the ideal $\mathfrak{a}$ of nowhere dense sets in a basic ring $A$ for a space $\Re$ determines a set of redundancy $\mathbb{E}(\mathfrak{a})$ in the algebraic map $m(\Re, \mathfrak{E}(A), \mathcal{X})$. The investigation of this aspect of the mapping theory leads to the introduction of a new type of topological space which proves to occupy a place intermediate between those already considered and the regular spaces. It is these new spaces which we propose to investigate systematically in the present section. We pass later to the study of more special types of space, including the regular spaces.

The formal definition of the spaces to be considered reads as follows:

Definition 19. A $T_{0 \text {-space }} \Re$ is said to be a semi-regular or to be an $S R$-space if the regular open sets constitute a basis for $\Re$.

The essential reason that the $S R$-spaces lead to simplification of the mapping theory is that the nowhere dense sets play a less important rôle than they do in other spaces. This fact is already emphasized in Theorems 24 and 25 , and is brought out yet more clearly in the following algebraic theorem.

THEOREM 60. If $A$ is a basic ring in an $S R$-space $\Re$, if $\mathfrak{a}$ is the ideal of all nowhere dense sets in $A$, and if $\mathfrak{a}_{\Re}$ is the class of all nowhere dense sets, then there exists a basic ring $A^{*}$ which is an isomorph of $A / \mathfrak{a}$ and a homomorph of $A$, the homomorphism $A \rightarrow A^{*}$ being defined by a correspondence $a \rightarrow a^{*}$ such that $a^{-^{\prime \prime}}<a^{*}<a^{\prime-\prime}, a \equiv a^{*}\left(\bmod \mathfrak{a}_{\mathfrak{R}}\right)$.

This theorem reposes essentially upon results given elsewhere by v. Neumann and the writer. $f$ If $B$ is the basic ring generated by $A$ and $a_{\Re}$, it is easily seen that the quotient-rings $A / \mathfrak{a}$ and $B / \mathfrak{a}_{\Re}$ are isomorphic: for each residual class $\left(\bmod a_{\Re}\right)$ in $B$ intersects $A$ in a corresponding residual class $(\bmod a)$. The construction of the desired $\operatorname{ring} A^{*}$ is therefore equivalent to the selection, from each residual class $\left(\bmod a_{\Re}\right)$ in $B$, of a representative element $a^{*}$ in such a manner that the representative elements constitute a subring

$\dagger$ v. Neumann and Stone, Fundamenta Mathematicae, vol. 25 (1935), pp. 353-378, especially Theorem 18. 
of $B$. If $a$ is any element of $A$ the correspondence $a \rightarrow a^{*}$, where $a^{*}$ is the representative of that residual class $\left(\bmod \mathfrak{a}_{\mathfrak{R}}\right)$ in $B$ which contains $a$, obviously defines a homomorphism $A \rightarrow A^{*}$; and it is evident that $A^{*}$ and $A / \mathfrak{a}$ are isomorphic. Thus we have to solve the $\left(B, \mathfrak{a}_{\mathfrak{R}}\right)$ representation problem, which was studied in general terms in the paper cited. A solution can be obtained by application of Theorem 18 of that paper. First, let us consider the functions $F(b)=b^{\prime-\prime}, G(b)=b^{-{ }^{\prime \prime}}$ defined for all elements $b$ in $B$. They are connected by the relation $F(b)=G^{\prime}\left(b^{\prime}\right)$. From Theorems 24 and 25 we find that $G(b)$ has the following properties: $G(b) \epsilon B, G(b) \equiv b\left(\bmod a_{\Re}\right), G(b)=G(c)$ if and only if $b \equiv c\left(\bmod \mathfrak{a}_{\mathfrak{R}}\right), G(b c)=G(b) G(c)$. Accordingly, $F(b)$ has the properties: $F(b) \epsilon B, F(b) \equiv b\left(\bmod \mathfrak{a}_{\Re}\right), F(b)=F(c)$ if and only if $b \equiv c\left(\bmod \mathfrak{a}_{\Re}\right)$, $F(b \vee c)=F(b) \vee F(c)$. Secondly, let us consider arbitrary non-void subclasses $\mathfrak{R}$ and $\mathfrak{D}$ in $\mathfrak{a}_{\mathfrak{R}}$ with the property that $c<d$ or, equivalently, $c^{\prime} d=0$ for all $c$ in $\mathbb{R}$ and all $d$ in $\mathfrak{D}$. If we denote by $a_{0}$ the union of all sets $c$ in $\mathfrak{R}$, it is evident that $c<a_{0}<d$ or, equivalently, $c^{\prime} a_{0}=a_{0}^{\prime} d=0$ for all $c$ in $\mathfrak{R}$ and all $d$ in $\mathfrak{D}$. Since $a_{0}<d$ for every $d$ in the non-void class $\mathfrak{D}$ and since $\mathfrak{D}$ contains only nowhere dense sets, $a_{0}$ is also nowhere dense, belonging therefore to $a_{\mathfrak{R}}$ and to $B$. Thus the hypotheses of the theorem on which we wish to rely are seen to be satisfied, and the $\left(B, \mathfrak{a}_{\mathfrak{R}}\right)$ representation problem has a solution. Furthermore, the actual construction of this solution shows that the representative $a^{*}$ assigned to the residual class $\left(\bmod a_{\Re}\right)$ containing a given element $a$ in $A$ satisfies the relations $F^{\prime}\left(a^{\prime}\right)<a^{*}<F(a)$ or, equivalently, $a^{--^{\prime}}<a^{*}<a^{\prime-\prime}$. The relation $a^{*} \equiv a\left(\bmod \mathfrak{a}_{\mathfrak{R}}\right)$ is evident. We may note that the elements 0 and $e=\Re$ in $A$ have the correspondents $0^{*}=0, e^{*}=e$ respectively, by virtue of these relations.

It remains for us to prove that $A^{*}$ is a basic ring for $\Re$, in other words, that the interiors of the sets in $A^{*}$ constitute a basis for $\Re$. If $(\mathcal{F}$ is any open set in the $S R$-space $\Re$ and $\mathfrak{r}$ any point of $\mathbb{B}$, then there exists a regular open set $\mathfrak{S}$ such that $\mathfrak{r} \in \mathfrak{S} \subset \mathcal{G}$. Since $A$ is a basic ring for $\Re$, it contains an element $a$ such that $\mathrm{r} \epsilon a^{\prime-^{\prime}} \subset \mathfrak{S}$. We shall show that the corresponding element $a^{*}$ satisfies the relations $\mathrm{r} \epsilon\left(a^{*}\right)^{\prime-^{\prime}} \subset$ B). Using the relations $a<a^{-}, a^{--^{\prime}}<a^{*}$, we see that $a^{\prime-^{\prime}}<\left(a^{-}\right)^{\prime-\prime}<a^{*}, a^{\prime-^{\prime}}=\left(a^{\prime-\prime}\right)^{\prime-\prime}<\left(a^{*}\right)^{\prime-\prime}$, and $\mathrm{r} \epsilon\left(a^{*}\right)^{\prime-\prime}$. Using similarly the relation $a^{*}<a^{\prime-1}$, we see that $\left(a^{*}\right)^{\prime-\prime}<\left(a^{\prime-1-}\right)^{\prime-\prime}=\left(a^{\prime-\prime}\right)^{--^{\prime}} \subset \mathfrak{S}^{--^{\prime}}$ $=\mathfrak{S} \subset \mathbb{S}$. Hence $\boldsymbol{r} \epsilon\left(a^{*}\right)^{\prime-\prime} \subset \mathfrak{B}$, and $A^{*}$ is a basic ring for $\Re$.

We are now prepared to study the Boolean maps of $S R$-spaces, beginning with the following fundamental result.

THEOREM 61. If $\Re$ is an $S R$-space, if $A$ is a basic ring for $\Re$, if $m(\Re, \mathfrak{B}, X)$ where $\mathfrak{B}=\mathfrak{E}(A)$ is the algebraic map defined by $A$, and if $\mathfrak{a}$ is the ideal of all nowhere dense sets in $A$, then $\mathbb{E}(\mathfrak{a})$ is a set of redundancy for the given map. 
If $A^{*}$ is the basic ring constructed in Theorem 60 , the suppression of $\mathfrak{E}(\mathfrak{a})$ from $m(\Re, \mathfrak{B}, X)$ yields a map equivalent to $m\left(\Re, \&\left(A^{*}\right), X^{*}\right)$; the latter map has the property that the family $X^{*}$ is densely distributed in $\mathbb{E}\left(A^{*}\right)$.

The proof that $\&(\mathfrak{a})$ is a set of redundancy rests upon Theorem 18 above and is similar to the proof of a like result in Theorem 40. In fact, if we put $\mathfrak{Q}=\Re$ and replace the ideal $\mathfrak{b}$ by the ideal $\mathfrak{a}$ in Theorem 40 , we see that $\mathfrak{E}(\mathfrak{a})$ is a set of redundancy if $\Re$ has the following property: $(\mathrm{P})$ if $\mathbb{H}$ is any open set in $\Re$ and $\mathfrak{r}$ any point in $\mathscr{B}$, then there exists an open set $\mathfrak{S}$ such that $\mathfrak{r} \in \mathfrak{S} \subset \mathbb{S}$ and such that any set differing from $\mathfrak{S}$ by a nowhere dense set has interior contained in $\$$. Evidently, an $S R$-space has the property (P): we first choose $\mathfrak{S}$ as a regular open set such that $\mathfrak{r} \in \mathfrak{S} \subset \mathbb{S}$; and, if $\mathfrak{F}$ is any nowhere dense set, we use the relations $\mathfrak{S} \Delta \mathfrak{F} \subset \mathfrak{S} \cup \mathfrak{F} \subset(\mathfrak{S} \cup \mathfrak{F})-$ and $\mathfrak{S} \cup \mathfrak{F} \equiv \mathfrak{S}\left(\bmod \mathfrak{a}_{\mathfrak{R}}\right)$ to show that $(\mathfrak{S} \Delta \mathfrak{F})^{\prime-\prime} \subset(\mathfrak{S} \cup \mathfrak{F})^{\prime-\prime} \subset(\mathfrak{S} \cup \mathfrak{F})^{--^{\prime}}=\mathfrak{S C}^{--^{\prime \prime}}=\mathfrak{S} \subset \mathcal{S}$ in accordance with Theorem 25 . It follows that $\mathbb{E}(\mathfrak{a})$ is a set of redundancy for the given map.

The proof that the suppression of $\mathbb{E}(\mathfrak{a})$ yields a map equivalent to $m\left(\Re, \&\left(A^{*}\right), \chi^{*}\right)$ is also similar to part of the proof of Theorem 40 . Since $A \rightarrow A / \mathfrak{a} \longleftrightarrow A^{*}$, Theorem 4 shows that the Boolean spaces $\mathbb{E}^{\prime}(\mathfrak{a})$ and $\mathbb{E}\left(A^{*}\right)$ are topologically equivalent in such a way that $a \rightarrow a^{*}$ implies the correspondence of the sets $\mathbb{E}(a) \mathbb{F}^{\prime}(\mathfrak{a})$ and $\mathbb{E}\left(a^{*}\right)$ under this equivalence. If $\mathfrak{r}$ is any point in $\Re$ and if $a \rightarrow a^{*}$, we shall show that the relations $\mathfrak{X}(\mathfrak{r}) \mathbb{E}^{\prime}(\mathfrak{a}) \subset \mathbb{E}(a) \mathbb{E}^{\prime}(\mathfrak{a})$ and $\mathfrak{X}^{*}(\mathfrak{r}) \subset \mathfrak{E}\left(a^{*}\right)$ are equivalent; and we can then infer that the topological equivalence between $\mathfrak{E}^{\prime}(\mathfrak{a})$ and $\mathfrak{E}\left(A^{*}\right)$ places $\mathfrak{X}(\mathfrak{r}) \mathbb{E}^{\prime}(\mathfrak{a})$ and $\mathfrak{X}^{*}(\mathfrak{r})$ in correspondence. First, let $\mathfrak{X}(\mathfrak{r}) \mathfrak{E}^{\prime}(\mathfrak{a}) \subset \mathfrak{E}(a) \mathfrak{E}^{\prime}(\mathfrak{a})$. Then $A$ contains an element $b$ such that $b \equiv a(\bmod \mathfrak{a}), \mathfrak{r} \in b^{\prime}-^{\prime}$. By Theorems 24 and 25 , we must have $b^{--^{\prime}}=a^{-^{\prime \prime}}$. The elements $a$ and $b$ have a common correspondent $a^{*}$ under the homomorphism $A \rightarrow A^{*}$. In view of the relation $a^{-^{\prime \prime}}<a^{*}$, we have $\mathfrak{r} \in b^{\prime-^{\prime}}<b^{-^{\prime-\prime}}=a^{-^{\prime-\prime}}<a^{*}, \mathfrak{r} \epsilon\left(a^{*}\right)^{\prime-^{\prime}}$, and $\mathfrak{X}^{*}(\mathfrak{r}) \subset \mathbb{E}\left(a^{*}\right)$. On the other hand, let $a \rightarrow a^{*}, \mathfrak{X}^{*}(\mathfrak{r}) \subset \mathbb{E}\left(a^{*}\right)$. If we use the relation $a^{\prime-^{\prime}} \equiv a\left(\bmod \mathfrak{a}_{\mathfrak{R}}\right)$ in conjunction with Theorems 24 and 25, we find that $\left(a^{\left.\prime-^{-}-\right)^{\prime-\prime}}=\left(a^{\prime-^{\prime}}\right)^{--^{\prime}}=a^{-^{\prime-1}}\right.$. Since $a^{*}<a^{\prime-1}$, we see that $r \in\left(a^{*}\right)^{\prime-\prime}<\left(a^{\prime-1}\right)^{\prime-\prime}=a^{-\prime-1}$ or, equivalently, $\mathfrak{r}_{\boldsymbol{q}}\left(\left(a^{\prime}\right)^{\prime-\prime}\right)^{-}$. The basic ring $A$ therefore contains an element $b$ such that $\mathrm{r} \epsilon b^{\prime-\prime}$ and $\left(a^{\prime}\right)^{\prime-\prime} b^{\prime-1}=0$. Using Theorem 24 , we infer from the relations $a^{\prime} \equiv\left(a^{\prime}\right)^{\prime-^{\prime}}\left(\bmod \mathfrak{a}_{\mathfrak{R}}\right), b \equiv b^{\prime-^{\prime}}\left(\bmod \mathfrak{a}_{\mathfrak{R}}\right)$ that $a^{\prime} b \equiv 0\left(\bmod \mathfrak{a}_{\mathfrak{R}}\right)$. Since $a^{\prime} b$ is an element of $A$, the last relation assumes the form $a^{\prime} b \equiv 0(\bmod \mathfrak{a})$. Hence we have $\mathbb{E}\left(a^{\prime} b\right) \subset \mathbb{E}(\mathfrak{a})$ or, equivalently, $\mathbb{E}(b) \subset \mathbb{E}(\mathfrak{a}) \cup \mathbb{E}(a)$. Since $\mathfrak{r} \in b^{\prime{ }^{\prime}}$ implies $\mathfrak{X}(\mathfrak{r}) \subset \mathfrak{F}(b) \subset \mathfrak{F}(\mathfrak{a}) \cup \mathfrak{F}(a)$, we conclude that $\mathfrak{X}(\mathfrak{r}) \mathfrak{F}^{\prime}(\mathfrak{a}) \subset \mathfrak{F}(a) \mathbb{F}^{\prime}(\mathfrak{a})$. In this way we complete the proof that the suppression of $\mathbb{E}(\mathfrak{a})$ yields a map equivalent to $m\left(\Re, \mathbb{E}\left(A^{*}\right), \chi^{*}\right)$. 
Finally we show that, in the terminology of Definition 4 , the family $X^{*}$ is densely distributed in $\mathbb{E}\left(A^{*}\right)$. Since the ideal $\mathfrak{a}^{*}$ of nowhere dense sets in $A^{*}$ consists of the void set alone, we have $\mathbb{E}^{\prime}\left(\mathfrak{a}^{*}\right)=\mathbb{E}\left(A^{*}\right)$. If $\mathfrak{p}^{*}$ is any point in $\&\left(A^{*}\right)$, Theorem 34 therefore shows that $\left\{p^{*}\right\}$ is an $X^{*}$-set. Consequently, if $\mathbb{S}^{*}$ is any non-void open subset of $\&\left(A^{*}\right)$, it contains not only a set $\left\{p^{*}\right\}$ but also a set $\mathfrak{X}^{*}$ belonging to the family $X^{*}$. This is what we wished to prove.

We now establish a converse of Theorem 61 .

THEOREM 62. If $\Re$ is a $T_{0^{-}}$space and if $m(\Re, \mathfrak{B}, \Upsilon)$ is any Boolean map with the property that $\Upsilon$ is densely distributed in $\mathscr{B}$, then $\Re$ is an $S R$-space; and the given map is equivalent to an algebraic map $m\left(\Re, \&\left(A^{*}\right), \chi^{*}\right)$ of the kind described in Theorem 61.

We may regard $\mathscr{B}$ as the representative of an abstract Boolean ring $B$, and may even take $\mathfrak{B}=\mathbb{E}(B)$ without loss of generality. The relations $\mathfrak{Y}(\mathfrak{r}) \subset \mathbb{E}(b), b \in B$, define an open subset $\mathbb{S}(b)$ in $\Re$. We shall show that $\mathbb{S}(b)$ is even a regular open set. To this end, we prove first that $\mathfrak{S}^{-1}(b)=\mathbb{S}\left(b^{\prime}\right)$. If $\mathfrak{r} \in(S)^{-1}(b)$, there exists an element $c$ in $B$ such that $\mathfrak{r} \in(S)$ and $\mathscr{S}(b) \mathscr{S}(c)=0$ :

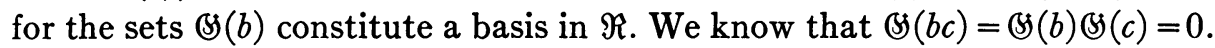
If $b c \neq 0$, the fact that $\Upsilon$ is densely distributed in $\&(B)$ would imply that the open set $\mathbb{E}(b c)$, being non-void, contains some set $\mathfrak{Y}$; and we could then infer that $\mathbb{S}(b c) \neq 0$. Thus $\mathbb{S}(b c)=0$ implies $b c=0$. Hence we see that $\mathfrak{Y}(\mathfrak{r}) \subset \mathbb{E}(c) \subset \mathbb{E}\left(b^{\prime}\right), \mathfrak{r} \in \mathbb{S}\left(b^{\prime}\right)$. On the other hand, if $\mathfrak{r} \in \mathbb{S}\left(b^{\prime}\right)$, the relations

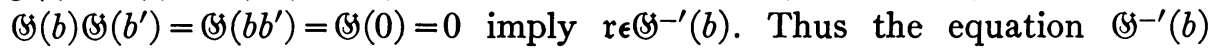
$=\$\left(b^{\prime}\right)$ is valid. It follows immediately that $\leftrightarrow(b)$ is a regular open set in accordance with the equations $\mathfrak{S}^{--^{-\prime}}(b)=\mathfrak{S}^{-1}\left(b^{\prime}\right)=\mathbb{S}\left(b^{\prime \prime}\right)=\mathfrak{S}(b)$. Since the sets $\mathfrak{S}(b), b \in B$, constitute a basis for $\Re$, this space must be an $S R$-space by Definition 19.

The Boolean ring $A$ generated by the sets $B(b), b \epsilon B$, is obviously a basic ring for $\Re$. If $a$ is the ideal of nowhere dense sets in $A$, we can show that $A / \mathfrak{a}$ is isomorphic to the abstract $\operatorname{ring} B$. The proof is based upon the relations

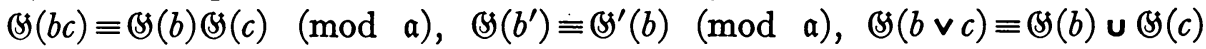
$(\bmod \mathfrak{a})$, and $\mathfrak{S}(b+c) \equiv \mathbb{S}(b) \Delta \mathfrak{S}(c)(\bmod \mathfrak{a})$. Since $\mathbb{S}(b c)=\mathbb{S}(b) \mathfrak{S}(c)$, the first of these congruences is trivial. The second is established as follows: Theorem 24 shows that $\mathfrak{S}^{\prime}(b) \equiv\left(\mathcal{S}^{\prime}(b)\right)^{\prime \prime \prime}\left(\bmod \mathfrak{a}_{\Re}\right)$ and the result noted above shows that $\mathbb{S}^{-\prime}(b) \equiv \mathfrak{B}\left(b^{\prime}\right)\left(\bmod \mathfrak{a}_{\mathfrak{R}}\right)$; combining these congruences we see that $\mathfrak{B}^{\prime}(b) \equiv \mathfrak{S}^{\prime}\left(b^{\prime}\right)\left(\bmod \mathfrak{a}_{\mathfrak{R}}\right)$; and, observing that $\mathfrak{S}^{\prime}(b)$ and $\mathfrak{S}^{\prime}\left(b^{\prime}\right)$ are both in $A$, we can rewrite the last congruence in the form $\mathfrak{S}^{\prime}\left(b^{\prime}\right) \equiv \mathcal{B}^{\prime}(b)(\bmod \mathfrak{a})$. The remaining congruences then follow algebraically from the first two. Any element $a$ in $A$ is expressible as a polynomial in terms of the sets $\$(b), b \in B$, and the operations - and $\Delta$, since the unit $e$ in $B$ has the property that $B(e)=\Re$. 
Consequently the congruences established above enable us to write $a \equiv \circlearrowleft(b)\left(\bmod a_{\Re}\right)$ for a suitable element $b$ in $B$. Moreover, this congruence becomes $a \equiv \circlearrowleft(b)(\bmod a)$ by virtue of the fact that $a$ and $\circlearrowleft(b)$ both belong to $A$. In this congruence the element $b$ is uniquely determined: for $\mathfrak{S}(b) \equiv \mathbb{S}(c)(\bmod \mathfrak{a})$ implies $\mathbb{S}(b+c) \equiv 0(\bmod \mathfrak{a})$; the fact that $\mathfrak{B}(b+c)$ is a regular open set then implies $\mathbb{S}(b+c)=0$; and it follows, as we observed above, that $b+c=0$ or, equivalently, $b=c$. The correspondence from $A$ to $B$ defined by putting $a \rightarrow b$ whenever $a \equiv \circlearrowleft(b)(\bmod a)$ is now seen to be a homomorphism: it is univocal; and the correspondences $a_{1} \rightarrow b_{1}, a_{2} \rightarrow b_{2}$ evidently imply $a_{1} a_{2} \equiv \mathfrak{S}\left(b_{1} b_{2}\right)(\bmod \mathfrak{a}), a_{1}+a_{2} \equiv \mathfrak{S}\left(b_{1}+b_{2}\right)(\bmod \mathfrak{a})$, or $a_{1} a_{2} \rightarrow b_{1} b_{2}$, $a_{1}+a_{2} \rightarrow b_{1}+b_{2}$. Since the relations $a \rightarrow 0$ and $a \equiv \mathfrak{S}(0) \equiv 0(\bmod \mathfrak{a})$ are equivalent, the ideal defined in $A$ by the homomorphism $A \rightarrow B$ coincides with a; and the rings $A / \mathfrak{a}$ and $B$ are therefore isomorphic.

By applying Theorem 36 we shall next prove that the map $m(\Re, \mathfrak{B}, \Upsilon)$ is equivalent to that obtained from $m(\Re, \mathbb{E}(A), X)$ by the suppression of the set $\mathbb{E}(\mathfrak{a})$; and Theorem 61 then justifies the assertion that $m(\Re, \mathfrak{B}, \Upsilon)$ is equivalent to an algebraic map $m\left(\Re, \&\left(A^{*}\right), X^{*}\right)$. First we recall that the isomorphism $A / \mathfrak{a} \longleftrightarrow B$ defines a topological equivalence between $\mathscr{F}^{\prime}(\mathfrak{a})$ and $\mathfrak{B}=\mathbb{E}(\boldsymbol{B})$ which places the sets $\mathbb{E}(a) \mathbb{F}^{\prime}(\mathfrak{a})$ and $\mathfrak{E}(b)$ in correspondence whenever the homomorphism $A \rightarrow B$ carries $a$ into $b$. Thus the topological equivalence may be described in the following explicit terms: if $\mathfrak{p}$ is any point in $\mathbb{F}^{\prime}(\mathfrak{a})$, its image in $\mathbb{E}(B)$ is the unique point $q$ which is common to all sets $\mathbb{E}(b)$ where $p \in \mathbb{E}(a)$ and $a \rightarrow b$; and, conversely, if $q$ is any point in $\mathbb{E}(B)$, its image in $\mathfrak{E}^{\prime}(\mathfrak{a})$ is the unique point $\mathfrak{p}$ common to all sets $\mathbb{E}(a)$ where $a \rightarrow b$ and $q \in \mathbb{E}(b)$. We now compare this correspondence between $\mathfrak{B}=\mathbb{E}(B)$ and $\mathbb{E}^{\prime}(\mathfrak{a})$ with the correspondence from $\Upsilon$-sets to $X$-sets constructed in Theorem 36 . By Theorem 34 , the minimal $X$-sets in $\mathbb{E}(A)$ are precisely the one-element subsets of $\mathscr{F}^{\prime}(\mathfrak{a})$; and, by virtue of the fact that $\Upsilon$ is densely distributed in $\mathfrak{B}$, the minimal $\Upsilon$-sets in $\mathbb{B}=\mathbb{E}(B)$ are precisely the one-element subsets of $\mathbb{E}(B)$. From the preceding description of the topological equivalence between $\xi^{\prime}(\mathfrak{a})$ and $\&(B)$, and from the information obtained in Theorem 36 , we therefore see that the two correspondences under discussion have the same effect upon the minimal sets: the topological equivalence places the minimal $X$-sets in biunivocal correspondence with the minimal $\Upsilon$-sets in a way which coincides with that described in Theorem 36. Since the correspondence of Theorem 36 carries $\mathfrak{V}(\mathfrak{r})$ into $\mathfrak{X}(\mathfrak{r})$ and preserves inclusion-relations, we infer that the topological equivalence places $\mathfrak{Y}(\mathfrak{r})$ in correspondence with $\mathfrak{X}(\mathfrak{r}) \mathfrak{S}^{\prime}(\mathfrak{a})$ : for the minimal $\Upsilon$-sets contained in $\mathfrak{V}(\mathfrak{r})$ are in correspondence with the minimal $X$-sets contained in $\mathfrak{X}(\mathfrak{r})$; and these two families of minimal sets have $\mathfrak{V}(\mathfrak{r})$ and $\mathfrak{X}(\mathfrak{r}) \mathfrak{E}^{\prime}(\mathfrak{a})$ as their respective unions. We have thereby proved that 
$m(\Re, \mathfrak{B}, \Upsilon)$ is equivalent to the map obtained from $m(\Re, \mathfrak{E}(A), X)$ by the suppression of the set $\mathfrak{E}(\mathfrak{a})$.

It is useful to summarize the results of Theorems 61 and 62 in the following terms:

THEOREM 63. The following properties of a $T_{0}$-space $\Re$ are equivalent:

(1) $\Re$ is an $S R$-space;

(2) $\Re$ has a Boolean map $m(\Re, \mathfrak{B}, \Upsilon)$, where $\Upsilon$ is densely distributed in $\mathfrak{B}$;

(3) $\Re$ has an algebraic map $m(\Re, \mathbb{E}(A), X)$ in which $\mathbb{E}(\mathfrak{a})$ is a set of redundancy.

If a space $\Re$ has any of these equivalent properties, then those of its Boolean maps $m(\Re, \mathfrak{B}, \Upsilon)$ which have the property of being densely distributed in $\mathfrak{B}$ are characterized as the irredundant algebraic maps of $\Re$; each such map can be constructed from a suitable algebraic map $m(\Re, \mathfrak{E}(A), X)$ by suppression of the corresponding set $\mathbb{E}(\mathfrak{a})$; and this construction, applied to an arbitrary algebraic map, yields a map of the indicated type.

With Theorem 63, the theory of maps for semi-regular spaces is brought to a satisfactory conclusion. That this development of the theory corresponds to a real rather than an apparent specialization is shown by the following result.

TheOREM 64. There exist $T_{0^{-}}, T_{1^{-}}$and $H$-spaces which are not $S R$-spaces.

The $T_{0}$-space discussed in Theorem 47 is neither a $T_{1}$-space nor an $S R$-space: we have already seen that it is not a $T_{1}$-space; and by reference to Theorem 61 we now see that it cannot be an $S R$-space. Similarly, the $T_{1}$-space discussed in Theorem 46 is neither an $H$-space nor an $S R$-space. A space described in AH provides an example of an $H$-space which is not an $S R$-space.* The points of this space $\Re$ are the real numbers $r, 0 \leqq r \leqq 1$. A neighborhood system is introduced in $\Re$ as follows: if $0<r_{0}<1$, the neighborhoods of $r_{0}$ are the sets $a<r<b$ where $0<a<r_{0}<b<1$; if $r=1$, the neighborhoods of $r$ are the sets $a<r \leqq 1$ where $0<a$; and if $r_{0}=0$, the neighborhoods of $r_{0}$ are the sets $B(a)$ consisting of all points $r$ such that $0 \leqq r<a$, $r \neq 1 / n$ where $a<1$ and $n=2,3,4, \cdots$. It is easily verified that $\Re$ is an $H$-space. If $\&$ is a regular open set containing the point 0 , then $\&$ contains some neighborhood $\mathbb{S}(a)$ of 0 . Since $B-(a)$ obviously consists of all points $r$ such that $0 \leqq r \leqq a$, we see that $\mathbb{S}^{-1-1}(a)$ consists of all points $r$ such that

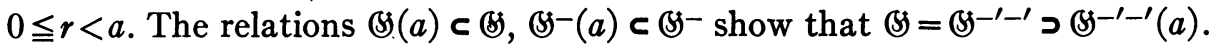
Hence $B$ is contained in no neighborhood of the point 0 . Thus $\Re$ is not an $S R$-space. We can show in addition that $\Re$ is absolutely closed with respect

* AH, p. 31, Beispiel 1. 
to immediate or strict $H$-extension. Indeed, if an infinite family of distinct open sets covers $\Re$, at least one of them contains a neighborhood $B(a)$ of 0 ; and the remaining sets cover the set $a \leqq r \leqq 1$. Since the topology of the subspace $a \leqq r \leqq 1$ is the usual topology, we see that the given family contains sets $\mathfrak{S}_{1}, \cdots, \mathfrak{S}_{n}$, where $\mathfrak{G}_{1} \supset \mathfrak{S}_{(a)}(a)$ and $\mathfrak{S}_{2}, \cdots, \mathfrak{S}_{n}$ cover the set $a \leqq r \leqq 1$. Thus the sets $\mathscr{G}_{1}^{-}, \cdots,{S_{n}}_{n}^{-}$cover $\Re$; and $\Re$ is absolutely closed with respect to immediate or strict $H$-extension in accordance with Theorem 50 .

The simplification of the mapping theory in the case of $S R$-spaces leads to a corresponding simplification of the theory of extensions. The chief result assumes the following form.

THEOREM 65. If a $T_{0 \text {-space }} \Re$ has an immediate extension $\mathfrak{Q}$ which is an $S R$-space, then $\Re$ is itself an $S R$-space and $\mathfrak{Q}$ is a strict extension of $\Re$. All $S R$-spaces $\mathfrak{Q}$ which are strict extensions of an $S R$-space $\Re$ can be obtained by the following construction: in a Boolean map $m(\Re, \mathfrak{B}, \Upsilon)$ where $\Upsilon$ is densely distributed in $\mathfrak{B}$, the family $\Upsilon$ is augmented by the adjunction of $\Upsilon$-sets to provide a family Z; and the familiar topology is then imposed upon Z. Conversely, this construction always yields an $S R$-space $\mathfrak{Q}$ which is a strict extension of $\Re$. The space $\mathfrak{Q}$ is a $T_{1}$-extension of $\Re$ if and only if no set in $Z-\Upsilon$ contains or is contained in any distinct set in $Z$; and $\mathfrak{Q}$ is an $H$-extension of $\Re$ if and only if no set in $Z-Y$ has points in common with any distinct set in $Z$.

If $\Re$ has an immediate extension $\mathfrak{Q}$ which is an $S R$-space, we consider the maps described in Theorem 40. If $\mathfrak{a}$ is the ideal of all nowhere dense sets in $A$, then the suppression of the set $\mathbb{E}(\mathfrak{a})$ from $m(\mathfrak{Q}, \mathbb{E}(A), Z)$ yields a map $m\left(\mathfrak{Q}, \mathfrak{E}^{\prime}(\mathfrak{a}), Z^{*}\right)$ in accordance with Theorem 61 . If we consider only those members of the families $Z$ and $Z^{*}$ which represent points of the subspace $\Re$, we obtain maps $m(\Re, \&(A), X)$ and $m\left(\Re, \mathbb{F}^{\prime}(\mathfrak{a}), X^{*}\right)$. It is evident that the second of these maps can be constructed from the first by the suppression of the set $\mathbb{E}(\mathfrak{a})$. We now show that $X^{*}$ is densely distributed in $\mathfrak{F}^{\prime}(\mathfrak{a})$. If $\mathfrak{p}$ is any point in $\mathcal{F}^{\prime}(\mathfrak{a})$, then $\{p\}$ is a $Z$-set in accordance with Theorem 34; but Theorem 40 shows that $\{p\}$ is also an $X$-set. Hence $p \in \mathbb{E}(a)$ implies the existence of a set $\mathfrak{X}(\mathfrak{r})$ in $X$ such that $\mathfrak{X}(\mathfrak{r}) \subset \mathbb{E}(a)$. Consequently $\mathfrak{p} \in \mathfrak{E}^{\prime}(\mathfrak{a}) \mathfrak{E}(a)$ implies the existence of a corresponding set $\mathfrak{X}^{*}(\mathfrak{r})=\mathfrak{X}(\mathfrak{r}) \mathbb{E}^{\prime}(\mathfrak{a})$ in $X^{*}$ such that $\mathfrak{X}^{*}(\mathfrak{r}) \subset \mathbb{F}^{\prime}(\mathfrak{a}) \mathfrak{F}(a)$, as we wished to prove. Since $X^{*}$ is densely distributed in $\mathscr{F}^{\prime}(\mathfrak{a})$, the existence of the map $m\left(\Re, \mathscr{F}^{\prime}(\mathfrak{a}), X^{*}\right)$ implies that $\Re$ is an $S R$-space in accordance with Theorem 62 . Furthermore the given extension $\mathfrak{Q}$ can evidently be obtained from the map $m\left(\Re, \mathbb{E}^{\prime}(\mathfrak{a}), X^{*}\right)$ by the construction described in the statement of the theorem. We can easily verify that $\mathscr{Q}$ is a strict extension of $\Re$ in accordance with Definition 14: if $q$ is any point of the $S R$-space $\mathfrak{Q}$ and $(\mathcal{S}$ any open set containing $\mathfrak{q}$, there exists a regular open 
set $\mathfrak{S}$ such that $\mathfrak{q} \in \mathfrak{S} \subset \mathfrak{G}$; and if $\mathfrak{F}$ is any nowhere dense set, not merely one which is contained in $\Re^{\prime}$, we have $(\mathfrak{g} \Delta \mathfrak{F})^{\prime-^{\prime}} \subset(\mathfrak{S} \Delta \mathfrak{F})^{-^{\prime \prime}}=\mathfrak{W}^{-^{\prime \prime}}=\mathfrak{W} \subset \mathfrak{S}$.

The construction described in the statement of the theorem yields an immediate extension $\mathfrak{Q}$ of $\Re$ in accordance with Theorem 38. Since the family $\Upsilon$ is assumed to be densely distributed in $\mathscr{B}$, the family $Z$ is also densely distributed in $\mathfrak{B}$; and the space $\mathfrak{Q}$ must therefore be an $S R$-space in accordance with Theorem 62 . It follows that $\mathfrak{Q}$ is a strict extension of $\Re$. The condition that $\mathfrak{Q}$ be a $T_{1}$-extension of $\Re$ has already been established in Theorem 38. So also has the sufficiency of the condition that $\mathfrak{Q}$ be an $H$-extension of $\Re$. We shall now prove that this condition is necessary: if $3(q) \epsilon Z-X$ and $\mathbb{Z}(\mathfrak{r}) \in Z$ have the property $\mathbb{Z}(\mathfrak{q}) \mathbb{Z}(\mathfrak{r}) \neq 0$, then any two open sets $\mathfrak{S}(\mathfrak{q})$ and $\mathfrak{S}(\mathfrak{r})$ in $\mathfrak{B}$ with the properties $\mathbb{Z}(\mathfrak{q}) \subset \mathfrak{S}(\mathfrak{q}), \mathbb{Z}(\mathfrak{r}) \subset \mathfrak{S}(\mathfrak{r})$ necessarily have the property $\mathfrak{S}(\mathfrak{q})(\mathfrak{S}(\mathfrak{r}) \neq 0$; and since $Z$ is densely distributed in $\mathfrak{B}$, there exists a set $\mathbb{Z}$ in $Z$ such that $\mathbb{Z} \subset \mathfrak{S}(\mathfrak{q}) \mathfrak{S}(\mathfrak{r})$. Accordingly the open sets $\mathfrak{E}(\mathfrak{q}), \mathfrak{S}(\mathfrak{r})$ specified in $\mathfrak{Q}$ by the respective relations $\mathfrak{B \subset G}(\mathfrak{q}), \mathfrak{B \subset G}(\mathfrak{r})$ have the properties $\mathfrak{q} \in \mathfrak{W}(\mathfrak{q}), \mathfrak{r} \in \mathfrak{G}(\mathfrak{r}), \mathfrak{S}(\mathfrak{q}) \mathfrak{W}(\mathfrak{r}) \neq 0$, where $\mathfrak{q} \in \Re^{\prime}$ and $\mathfrak{r} \in \mathfrak{Q}$. Since the sets $\mathfrak{S}$ specified by $\mathbb{Z} \subset \mathbb{B}$ constitute a basis for $\mathfrak{Q}$, the points $\mathfrak{q}$ and $\mathfrak{r}$ obviously do not have the $H$-separation property; and $\mathfrak{Q}$ is not an $H$-extension of $\Re$. This completes the proof.

It is also easy to specialize Theorem 52 for the case of $S R$-spaces. We obtain the following result.

THEOREM 66. Every $S R$-space $\Re$ of infinite character c has a strict $H$-extension $\mathfrak{Q}$ which has character $\mathfrak{c}$, which is absolutely closed with respect to immediate or strict $H$-extension, and which is an $S R$-space. The points adjoined to $\Re$ in this extension may be assumed to constitute a totally-disconnected $H$-space.

We first construct a Boolean map $m(\Re, \mathfrak{B}, \Upsilon)$ where $\mathfrak{B}$ has character $\mathfrak{c}$ and $\Upsilon$ is densely distributed in $\mathscr{B}$. We start this construction by selecting a basis of cardinal number $\mathrm{c}$ for $\Re$. The basic ring $A$ generated by this basis evidently has cardinal number $\mathfrak{c}$; and the representative Boolean space $\mathbb{E}(A)$ has character $\mathfrak{c}$. The suppression of the set $\mathbb{E}(\mathfrak{a})$ from $\mathbb{E}(A)$ therefore leaves a Boolean space $\mathbb{F}^{\prime}(\mathfrak{a})$ of character not exceeding $\mathfrak{c}$. Theorem 61 then shows that we can obtain the desired map $m(\Re, \mathfrak{B}, \Upsilon)$ by taking $\mathfrak{B}=\mathfrak{E}^{\prime}(\mathfrak{a})$. According to Theorem 23 , the character of $\Re$ cannot exceed that of $\mathscr{F}^{\prime}(\mathfrak{a})$. Hence the character of $\mathbb{E}^{\prime}(\mathfrak{a})$ must be equal to $c$. Now we apply the construction of Theorem 65 to obtain the desired extension $\mathfrak{Q}$ by means of the map $m(\mathfrak{Q}, \mathfrak{B}, Z)$. The family $Z$ is formed by adjoining to $\Upsilon$ all one-element subsets $\{p\}$ in $\mathscr{B}$, where $p$ belongs to none of the sets in $\Upsilon$. Theorems 62 and 63 show that $m(\mathfrak{Q}, \mathfrak{B}, Z)$ is an algebraic map; by construction, the family $Z$ covers $\mathfrak{B}$; and hence Theorem 49 shows that $\mathfrak{Q}$ is absolutely closed with respect to 
immediate or strict $H$-extension. By the reasoning given in the proof of Theorem 52 , the points adjoined to $\Re$ in this extension constitute a totallydisconnected $H$-space.

In conclusion, we remark upon the connections between the results of the present section and those given in Theorem 29. By comparing Theorems 29, 61,62 , and 63 , we are able to state the following result.

THEOREM 67. If $\Re$ is a $T_{0}$-space and $\Re^{*}$ the associated continuous image constructed in Theorem 29, then $\Re^{*}$ is an $S R$-space. If $\Re$ is itself an $S R$-space, then $\Re$ and $\Re^{*}$ are topologically equivalent.

2. Regular spaces. We pass now to the consideration of a stronger separation property which has long played an important rôle in general topology. We introduce it through one of the standard definitions.

Definition 20. A $T_{0}$-space is said to be regular or to be an $\Re$-space if, whenever $\mathbb{S}$ is an open set in $\Re$ and $\mathfrak{r}$ is a point in $\mathfrak{S}$, there exists an open set $\mathfrak{E}$ such that $\mathfrak{r} \in \mathfrak{S}, \mathfrak{S}^{-} \subset \mathfrak{S}$.

The relative strength of this separation property is disclosed by the following theorem.

ThEOREM 68. An R-space is both an $S R$-space and an $H$-space.

That an $R$-space is an $S R$-space is evident: for, if $\mathfrak{S}$ is the open set of Definition 20, the regular open set $\mathfrak{S}^{-\prime-\prime}$ has the properties $\mathfrak{r} \in \mathfrak{S}=\mathfrak{S}^{\prime-\prime} \subset \mathfrak{G}^{-\prime-\prime}$, $\mathfrak{S}^{-\prime-1} \subset \mathfrak{S}^{\prime-\prime}=\mathbb{S}$. In order to prove that an $R$-space is an $H$-space, we proceed as follows: if $\mathfrak{p}$ and $q$ are distinct points in $\Re$, the assumption that $\Re$ is a $T_{0^{-}}$ space implies that, the notation being properly chosen, $p$ is contained in an open set to which $q$ does not belong or, equivalently, that $p \in\{q\}^{-\prime}$; hence there exists an open set $\mathfrak{S}$ such that $\mathfrak{p} \in \mathscr{S}, \mathfrak{S}^{-} \subset\{q\}^{-\prime}$; and the points $\mathfrak{p}$ and $\mathfrak{q}$ therefore have the $H$-separation property in accordance with the relations $\mathfrak{p} \in \mathfrak{S}$, $q \in \mathfrak{S E}^{-\prime}, \mathfrak{S} \mathfrak{E}^{-\prime}=0$.

In view of Theorem 68, the simplified form of the mapping theory developed for $S R$-spaces applies in particular to $R$-spaces. It is possible, however, to carry the study of $R$-spaces somewhat further than this.

Theorem 69. If a $T_{0}$-space $\Re$ has a continuous Boolean map $m(\Re, \mathfrak{B}, \Upsilon)$, then $\Re$ is an $R$-space. Conversely, if $\Re$ is an $R$-space, its irredundant algebraic maps $m(\Re, \mathbb{E}(A), X)$, and likewise its general algebraic maps $m(\Re, \&(A), X)$ where $A$ contains a basis for $\Re$, are all continuous maps.

Let $m(\Re, \Re, \Upsilon)$ be a continuous Boolean map of a $T_{0}$-space $\Re$. If $\mathbb{B}$ is an open set in $\Re$ and $\mathfrak{r}$ is any point in $\mathscr{G}$, then there exists a set $\mathfrak{S}_{1}$ which has the properties $\mathfrak{r} \in \mathfrak{H}_{1} \subset \mathscr{G}$ and which is specified by a bicompact open set $\mathfrak{F}_{1}$ in $\mathfrak{B}$ through the relations $\mathfrak{V} \subset \mathfrak{F}_{1}, \mathfrak{Y}(\mathfrak{r}) \subset \mathfrak{F}_{1}$ : for the sets so specified constitute a 
basis for $\Re$ in accordance with Theorem 23. Since the given map is continuous, there exists an open set $\mathfrak{F}_{0}$, which we may assume also to be bicompact, such that $\mathfrak{Y}(\mathfrak{r}) \subset \mathfrak{F}_{0}$ and such that $\mathfrak{Y} \mathfrak{F}_{0} \neq 0$ implies $\mathfrak{Y} \subset \mathfrak{F}_{1}$. We denote by $\mathfrak{S}$ the open set in $\Re$ specified by the relation $\mathfrak{V} \subset \mathfrak{F}_{0}$. It is obvious that $\mathfrak{r} \in \mathfrak{S} \subset \mathfrak{H}_{1} \subset \mathfrak{S}$. The set $\mathfrak{F}_{0}^{\prime}$ is also a bicompact open set in $\mathscr{B}$. The open set specified in $\Re$ by the relation $\mathfrak{V} \subset \mathfrak{F}_{0}^{\prime}$ has as its complement a closed set $\mathfrak{F}$ which is described by the relation $\mathfrak{V F}_{0} \neq 0$. Hence we have $\mathfrak{S} \subset \mathfrak{F} \subset \mathfrak{G}_{1} \subset \mathfrak{G}$. Since $\mathfrak{S}^{-} \subset \mathfrak{F}^{-}=\mathfrak{F}$, the open set $\mathfrak{S}$ satisfies the relations $\mathfrak{r} \in \mathfrak{S}, \mathfrak{S}^{-} \subset \mathfrak{S}$. The space $\Re$ is therefore an $R$-space.

If $m(\Re, \mathfrak{F}(A), X)$ is an algebraic map of an $R$-space $\Re$, let $\mathfrak{X}(\mathfrak{r})$ be an arbitrary member of the family $X$ and let $\&$ be an open subset of $\mathbb{E}(A)$ which contains $\mathfrak{X}(\mathfrak{r})$. Then there exists an element $a$ in $A$ such that $\mathfrak{X}(\mathfrak{r}) \subset \mathbb{E}(a) \subset \mathcal{B})$, $\mathfrak{r} \epsilon a^{\prime{ }^{\prime}}$. By hypothesis $\Re$ contains an open set $\mathfrak{E}$ such that $\mathfrak{r} \in \mathfrak{S}, \mathfrak{S}^{-} \subset a^{\prime{ }^{\prime}}$. The basic ring $A$ then contains an element $b$ such that $\mathfrak{r} \in b^{\prime-\prime} \subset \mathfrak{S}$. If we could use the equation $b^{\prime-^{-}-}=b^{-}$, we could conclude that $b^{-}=b^{\prime-^{\prime}} \subset \mathfrak{\mathfrak { E } ^ { - }} \subset a^{\prime-^{\prime}}$; and, by virtue of Theorem 28 , that $\mathfrak{X} \mathbb{E}(b) \neq 0$ implies $\mathfrak{X} \subset \mathbb{E}(a)$. Since $\mathfrak{r} \in b^{\prime-\prime}$ implies $\mathfrak{X}(\mathfrak{r}) \subset \mathfrak{E}(b)$ we could then show that the element $c=a b$ has the following properties: $\mathfrak{X}(\mathfrak{r})$ is contained in $\mathfrak{E}(c)=\mathfrak{E}(a) \mathfrak{E}(b) \subset \mathfrak{S}$; and $\mathfrak{X} \mathbb{E}(c) \neq 0$ implies $\mathfrak{X}(b) \neq 0$ and hence $\mathfrak{X} \subset \mathbb{E}(a) \subset \mathbb{B}$. The family $X$ would thus be continuous in accordance with Definition 5 , the map $m(\Re, \mathfrak{E}(A), X)$ continuous in accordance with Definition 7 . We therefore consider the conditions under which we can assert that $b^{\prime \prime^{-}}=b^{-}$. If $A$ contains a basis for $\Re$, we may take $b$ as an open set; and we then have $b^{\prime{ }^{\prime}}=b, b^{\prime{ }^{\prime}-}=b^{-}$. Again, if the map $m(\Re, \&(A), \mathcal{X})$ is irredundant, Theorems 63 and 68 show that $X$ is densely distributed; and the proof of Theorem 62 then shows that the equation $b^{\prime-1}=\mathbb{B}(b)$, which is valid because both members are specified by the relation $\mathfrak{X} \subset \mathfrak{E}(b)$, implies $b^{\prime-^{\prime}-}=\left(\mathfrak{S}^{-}-(b)=\left(\mathfrak{S}^{-\prime}(b)\right)^{\prime}=\left(\mathfrak{B}^{\prime}\left(b^{\prime}\right)=\left(\left(b^{\prime}\right)^{\prime-\prime}\right)^{\prime}=b^{-}\right.\right.$. Thus the map $m(\Re, \&(A), X)$ is continuous under either of the conditions stated in the theorem.

By combining Theorem 69 with Theorem 22, we obtain the following result.

TheOREM 70. In order that the algebraic map $m(\Re, \&(A), X)$ define a continuous univocal correspondence $\mathfrak{X}(\Re) \rightarrow \mathfrak{r}$ from the subspace $\mathfrak{S}(X)=\sum_{\mathrm{re \Re}} \mathfrak{X}(\mathfrak{r})$ of $\&(A)$ to the $T_{0}$-space $\Re$, it is necessary and sufficient that $\Re$ be an $R$-space and $m(\Re,(E), X)$ a continuous map. All $R$-spaces are thus found among the continuous images of subspaces of bicompact Boolean spaces.

It is now possible to recover from the mapping theory a well-known elementary criterion for bicompactness. ${ }^{*}$

* AH, pp. 91-92. 
TheOREM 71. In order that an $H$-space be bicompact, it is necessary and sufficient that it be an $R$-space absolutely closed with respect to immediate or strict $H$-extension.

We have already noted in Theorem 53 that a bicompact $H$-space is absolutely closed with respect to immediate or strict $H$-extension. We can now show that a bicompact $H$-space $\Re$ is an $R$-space. If $B$ is any open set in $\Re$ and $\mathfrak{r}$ is any point in $\mathfrak{B}$, there exist open sets $\mathfrak{S}(\mathfrak{p}), \mathfrak{F}(\mathfrak{p})$ corresponding to an arbitrary point $\mathfrak{p}$ in $\mathfrak{G}^{\prime}$ such that $\mathfrak{p} \in\left(\mathfrak{S}(\mathfrak{p}), \mathfrak{r} \in \mathfrak{S}(\mathfrak{p}), \mathfrak{S}(\mathfrak{p}) \mathfrak{S}(\mathfrak{p})=0\right.$. Since $\mathfrak{S}^{\prime}$ is closed, it is bicompact; and there exist points $\mathfrak{p}_{1}, \cdots, \mathfrak{p}_{n}$ in $\mathscr{B}^{\prime}$ such that $\mathfrak{S}\left(\mathfrak{p}_{1}\right) \cup \ldots \cup \mathfrak{S}\left(\mathfrak{p}_{n}\right) \supset \mathfrak{S}^{\prime}, \mathfrak{r} \in \mathfrak{S}\left(\mathfrak{p}_{1}\right) \cdots \mathfrak{S}\left(\mathfrak{p}_{n}\right) \subset \mathfrak{S}^{\prime}\left(\mathfrak{p}_{1}\right) \ldots \mathfrak{S}^{\prime}\left(\mathfrak{p}_{n}\right) \subset \mathfrak{S}$. If we define $\mathfrak{S}$ as the open set $\mathfrak{S}=\mathfrak{S}\left(\mathfrak{p}_{1}\right) \cdots \mathfrak{S}\left(\mathfrak{p}_{n}\right)$, we find that $\mathfrak{r} \in \mathfrak{S}, \mathfrak{S}^{-} \boldsymbol{C}\left(\mathfrak{S}^{\prime}\left(\mathfrak{p}_{1}\right) \cdots\right.$ $\left(\mathcal{S}^{\prime}\left(\mathfrak{p}_{n}\right)\right)$ - $\boldsymbol{c} \mathfrak{S}$. On the other hand, if $\Re$ is an $R$-space absolutely closed with respect to immediate or strict $H$-extension, we construct an irredundant algebraic map $m(\Re, \mathbb{E}(A), \mathcal{X})$ in accordance with Theorem 61 . Since $\Re$ is absolutely closed in the indicated sense, the family $X$ covers $\mathbb{E}(A)$ in accordance with Theorem 49 . Since $\Re$ is an $R$-space, Theorems 69 and 70 show that $\Re$ is a continuous image of the space $\mathbb{E}(A)=\mathfrak{S}(X)$. It follows from the bicompactness of $\mathfrak{E}(A)$ that $\Re$ is bicompact. In fact, any family of open sets which covers $\Re$ has a family of antecedents which are open and cover $\mathbb{E}(A)$; and, since a finite number of these antecedents suffices to cover $\&(A)$, a finite number of sets in the given family likewise suffices to cover $\Re$.

The proof just given for Theorem 71 can evidently be rearranged in such a way as to establish the following result, which we give without further formal discussion.

Theorem 72. Among all $H$-spaces, the bicompact spaces are characterized topologically as the continuous images of bicompact Boolean spaces. ${ }^{*}$

As a consequence of Theorem 71 we can now show that the $S R$-separation property is essentially weaker than the $R$-separation property.

THeorem 73. There exists an SR-space which is an $H$-space but not an $R$-space. Such a space may be constructed so as to be absolutely closed with respect to immediate or strict $H$-extension.

Let us suppose, on the contrary, that every space which is both an $S R$-space and an $H$-space is necessarily an $R$-space. If $\Re$ is an arbitrary $R$-space, we apply Theorem 66 to construct an immediate $H$-extension $\mathfrak{Q}$ of $\Re$ which is an $S R$-space absolutely closed with respect to immediate or strict $H$-extension. Theorem 42 shows that the extension $\mathfrak{Q}$ so constructed is an

\footnotetext{
* See the material related to this result in AH, pp. 95-98.
} 
$H$-space. By hypothesis, $\mathfrak{Q}$ is therefore an $R$-space. Theorem 71 therefore implies that $\mathfrak{Q}$ is bicompact. Thus our initial assumption leads to the following proposition: every $R$-space can be imbedded as a subspace in a bicompact $H$-space. Now an example due to Tychonoff* shows that this proposition is false. Whenever $\Re$ is restricted to have character $\boldsymbol{\aleph}_{0}$, however, the proposition is known to be true. Hence, if we start our construction with the $R$-space $\Re$ given by Tychonoff, we obtain a space $\mathfrak{Q}$ of the type described in the theorem. The character of the space $\mathfrak{Q}$, being equal to that of $\Re$, must exceed $\boldsymbol{\aleph}_{0}$.

We can rid ourselves of this restriction on the character of $\mathfrak{Q}$ by following a different process of construction. The Cantor discontinuum $\mathfrak{D}$ is a bicompact Boolean space of character $\boldsymbol{\aleph}_{0}$. In $\mathfrak{D}$ we introduce a family $X$ of disjoint closed sets as follows: first we form the two-element sets $\mathfrak{X}_{n}$ consisting of the points $x=3^{-n}=2 \sum_{\alpha=n+1}^{\infty} 3^{-\alpha}, x=1-3^{-n}=2 \sum_{\alpha=1}^{n} 3^{-\alpha}$ for $n=1,2,3, \cdots$, respectively; and then we form all the one-element sets $\{x\}$ where $x$ belongs to no set $\mathfrak{X}_{n}$. We see at once that the family $\mathcal{X}$ is densely distributed in $\mathfrak{D}$ but is not continuous. On topologizing the family $X$ in the usual way, we obtain a space $\mathfrak{Q}$ and a Boolean map $m(\mathfrak{Q}, \mathfrak{D}, \mathcal{X})$. The character of $\mathfrak{Q}$ is $\boldsymbol{\aleph}_{0}$. Theorem 63 shows that $\mathfrak{Q}$ is an $S R$-space and that the map $m(\mathfrak{Q}, \mathfrak{D}, X)$ is an irredundant algebraic map. Since $X$ covers $\mathfrak{D}$, Theorem 49 shows that $\mathfrak{Q}$ is absolutely closed with respect to immediate or strict $H$-extension. Since the sets in $\mathcal{X}$ are disjoint, the space $\mathfrak{Q}$ is an $H$-space. Theorem 69 shows finally that $\mathfrak{Q}$ is not an $R$-space. A similar construction could evidently be carried out in an arbitrary bicompact Boolean space of infinite character.

In conclusion we may pose the problem of discovering what restrictions upon $S R$-spaces imply regularity. We have seen above that certain obvious restrictions are not sufficient. In this connection, it is natural to consider the following question: what $S R$-spaces have the property that every subspace is also an $S R$-space; and, in particular, what $H$-spaces have this property? From Theorem 65, we know that all everywhere dense subspaces of an $S R$-space are $S R$-spaces. The problem therefore reduces to the restricted problem concerning closed subspaces alone.

3. Completely regular spaces. As we recalled at the close of the preceding section, there exist regular spaces which cannot be imbedded in bicompact $H$-spaces. Consequently any separation property which characterizes the subspaces of bicompact $H$-spaces must be stronger than the property of regularity. Such a stronger property was discovered by Tychonoff, who termed it "complete regularity." * We propose to investigate the influence of

\footnotetext{
* Tychonoff, Mathematische Annalen, vol. 102 (1930), pp. 544-561.
} 
this property upon the mapping theory. First, let us state the fundamental definition and some of its elementary consequences.

Definition 21. A $T_{0 \text {-space }} \Re$ is said to be completely regular or to be a $C R$-space if it has any one of the three equivalent properties:

(1) if $\mathfrak{F}$ is any closed set in $\Re$ and $\mathfrak{r}_{0}$ is any point in $\mathfrak{F}^{\prime}$, then there exists a continuous real function $f$ defined in $\Re$ such that $f\left(\mathfrak{r}_{0}\right)=0, f(\mathfrak{r})=1$ when $\mathfrak{r} \in \mathfrak{F}, 0 \leqq f(\mathfrak{r}) \leqq 1$

(2) the open sets $\Re(\alpha<f<\beta)$, where $\alpha$ and $\beta$ are arbitrary real numbers and $f$ is an arbitrary bounded continuous real function in $\Re$, constitute a basis for $\Re$;*

(3) if $\mathbb{B}$ is any open set in $\Re$ and $\mathfrak{r}_{0}$ is any point in $\mathbb{B}$, then there exists a family of open sets $\mathfrak{S}_{\tau}$ defined for all rational numbers $\tau, 0<\tau<1$, such

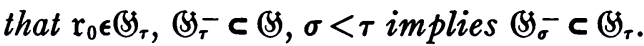

The equivalence of these three conditions is proved by well-known arguments, which we sketch briefly. First, (1) implies (2): if $\mathfrak{r}_{0} \in \mathfrak{S}$, we take the function $f$ corresponding to the closed set $\mathfrak{F}=\mathfrak{S B}^{\prime}$ and the point $\mathfrak{r}_{0}$ as described in (1) and observe that $\mathbb{S}_{0}=\Re(-1 / 2<f<1 / 2)$ has the properties $\mathfrak{r}_{0} \in \mathbb{S}_{0} \subset \mathbb{S}$. Similarly, (1) implies (3): if $\mathfrak{r}_{0} \in(\mathfrak{S}$, we again take $f$ as the function corresponding to $\mathfrak{F}=\mathbb{S}^{\prime}$ and $\mathfrak{r}_{0}$, and put $\mathfrak{S}_{\tau}=\Re(f<\tau), 0<\tau<1$. Next (2) implies (1): if $\mathfrak{r}_{0} \in \mathfrak{F}^{\prime}$, there exists an open set $\Re(\alpha<g<\beta)$ as described in (2) such that $\mathfrak{r}_{0} \in \Re(\alpha<g<\beta) \subset \mathfrak{F}^{\prime}$; and, if we put $\gamma=\min \left(g\left(\mathfrak{r}_{0}\right)-\alpha, \beta-g\left(\mathfrak{r}_{0}\right)\right) \neq 0$, $f(\mathfrak{r})=\min \left(1,\left|g(\mathfrak{r})-g\left(\mathfrak{r}_{0}\right)\right| / \gamma\right)$, we find that $f$ has the properties demanded in (1). Finally (3) implies (1). Taking $\mathfrak{B}=\mathfrak{F}^{\prime}$, we form the family $\mathfrak{S}_{\tau}, 0<\tau<1$, corresponding to $\mathbb{S}$ and $\mathfrak{r}_{0}$ in accordance with (3); and we define $\mathfrak{S}_{\tau}=0$ for rational numbers $\tau, \tau<0$, and $\mathfrak{S}_{\tau}=\Re$ for rational numbers $\tau, \tau>1$. We then define $f(\mathfrak{r})$ as the greatest lower bound of the numbers $\tau$ such that $\mathfrak{r} \in \mathscr{S}_{r}$. It is evident that $f\left(\mathfrak{r}_{0}\right)=0,0 \leqq f(\mathfrak{r}) \leqq 1, f(\mathfrak{r})=1$ in $\mathfrak{G}^{\prime}=\mathfrak{F}$. To prove that $f$ is continuous, we must show that $\Re(\alpha<f<\beta)$ is an open set. If $\mathfrak{r}$ is any point in this set, there exist rational numbers $\rho, \sigma, \tau$ such that $\alpha<\sigma<\rho<f(\mathfrak{r})<\tau<\beta$. We then have $\mathfrak{r} \in \mathbb{S}_{\rho}^{\prime} \mathbb{B}_{\tau} \subset \mathbb{G}_{\sigma}{ }^{\prime} \mathbb{S}_{\tau}$. On the other hand, if $\mathfrak{r} \in \mathbb{S}_{\sigma}{ }^{\prime} \mathbb{G}_{\tau}$ where $\alpha<\sigma<\tau<\beta$, we see that $\mathfrak{r}_{\Leftrightarrow} \mathbb{S}_{\sigma}, \mathfrak{r} \in \mathbb{S}_{\tau}$, hence that $\sigma \leqq f(\mathfrak{r}) \leqq \tau$, and hence that $\alpha<f(\mathfrak{r})<\beta$. Thus $\Re(\alpha<f<\beta)$ is the union of the open sets $\xi_{\sigma}{ }^{\prime} \mathcal{G}_{\tau}$ where $\alpha<\sigma<\tau<\beta$; and $\Re(\alpha<f<\beta)$ is also open, as we wished to prove.

We remark that the condition (3) can be expressed, without reference to the rational numbers, in terms of order alone. For this purpose, we introduce the concept of strong inclusion: the set $\mathfrak{F}_{1}$ is said to be strongly included in the set $\mathfrak{F}_{2}$, if $\mathfrak{F}_{1}^{-} \subset \mathfrak{F}_{2}$. Then we may replace (3) by the equivalent $\left(3^{\prime}\right):$ if $\mathfrak{r}_{0} \in \mathfrak{B}$,

* By $\Re(P)$ we mean the set of all points in $\Re$ which have the property $P$, here the property $\alpha<f<\beta$. 
there exists a countable family of open sets which contain $\mathfrak{r}_{0}$, which are contained in $\mathbb{B}$, and which are simply ordered by the relation of strong inclusion in such a way that there is no first, no last, and no pair without an intermediate third. The property $\left(3^{\prime}\right)$ may therefore be taken as characterizing the completely regular spaces in a purely topological manner. In practice, the properties (1) and (2) are the ones which are technically useful.

The relation of $C R$-spaces to other types of space is indicated in the following theorem.

THEOREM 74. A CR-space is an R-space, and hence both an $S R$ - and an $H$-space; every normal space is a CR-space. Every subspace of a CR-space is a $C R$-space. There exist $R$-spaces which are not $C R$-spaces, and $C R$-spaces which are not normal spaces.

From Definition 21 (3), it is evident that every $C R$-space is an $R$-space in accordance with Definition 20. Theorem 68 then shows that a $C R$-space is both an $S R$-space and an $H$-space. The proof that every normal space is a $C R$-space is given by establishing property (3) of Definition 21 ; the explicit construction of the family $\mathcal{G}_{\tau}$ is a familiar one.* By use of Definition 21 (1), it is easily seen that every subspace of a $C R$-space is also a $C R$-space. Examples given by Tychonoff justify the final statement of the theorem. $\dagger$

Since the definition of $C R$-spaces involves, directly or indirectly, the topological relations between general topological spaces and the real number system, it is clear that we cannot investigate them without injecting new methods into the discussion. In order to continue the algebraic tendencies of the present paper and also in order to obtain the maximum of new information, we propose to study the topological ring of all bounded continuous real functions in an arbitrary $T_{0}$-space $\Re$. We shall find that the mapping theory for $C R$-spaces is closely connected with the theory of the corresponding function-rings. Moreover, we shall find that this connection enables us to give a full account of the problem of imbedding $C R$-spaces as everywhere dense subspaces in bicompact $H$-spaces. In the subsequent discussion we shall denote the real number field by the letter $R$; and we shall not distinguish between this field and its isomorphs.

TheOREM 75. If $\mathfrak{M}$ is the class of all bounded continuous real functions defined in a $T_{0}$-space $\Re$, if the sum and product of such functions are defined in the usual way, and if the norm $\|f\|$ of a function $f$ is defined as the least upper bound of the numbers $|f(\mathfrak{r})|, \mathfrak{r} \in \Re$, then $\mathfrak{M}$ is a topological ring in the following sense:

* See AH, p. 74.

† Tychonoff, Mathematische Annalen, vol. 102 (1930), pp. 544-561. 
(1) under the operations of addition and multiplication, $\mathfrak{M}$ is a commutative ring containing $R$ as a subring;

(2) if $\|f-g\|$ is introduced as the distance between $f$ and $g$, then $\mathfrak{M}$ is a complete metric space;

(3) in the metric topology of (2), the ring operations are continuous, the polynomials $f+g, f g$ being continuous functions of their arguments $f$ and $g$.

If multiplication by $f$ be restricted to the case where $f \epsilon R$, then $\mathfrak{M}$ is a Banach space. The operation of forming the absolute value $|f|$ of a function $f$ in $\mathfrak{M}$ is continuous in the metric topology of $\mathfrak{M}$. Any subring of $\mathfrak{M}$ which contains $\alpha f$ together with $f$ whenever $\alpha \epsilon R$ and which is a closed subset of $\mathfrak{M}$ has the property that it contains $|f|$ together with $f$.

The verification of the statements made in this theorem may be left to the reader, as the detailed proofs are all familiar from elementary analysis. We may point out that metric convergence of a sequence in $\mathfrak{M}$ is equivalent to uniform pointwise convergence of the function-sequence in $\Re$. The final statement of the theorem involves an application of the Weierstrass approximation theorem: if $\alpha=\|f\|$, there exists a polynomial $p_{n}(x)$ such that ||$x\left|-p_{n}(x)\right| \leqq 1 / n$ for $-\alpha \leqq x \leqq \alpha$ and $p_{n}(0)=0$; and it follows that $\left\||f|-p_{n}(f)\right\| \leqq 1 / n, p_{n}(f)=\sum_{\nu=1}^{N^{(n)}} \alpha_{\nu}(n) f^{\nu}$. For general discussions of the subject matter of the present theorem, the reader is referred to the literature. $\dagger$

We shall find it convenient to introduce a few special descriptive terms for later use.

DEFINITION 22. The ring $\mathfrak{M}$ of Theorem 75 is called the function-ring of the space $\Re$. A closed subring of $\mathfrak{M}$ which contains the subfield $R$ is called an analytical subring of $\mathfrak{M}$. If $\mathfrak{N}$ and $\mathfrak{N}^{*}$ are homomorphic subrings of function-rings $\mathfrak{M}$ and $\mathfrak{M}^{*}$ respectively with the property that $f \rightarrow f^{*}$ implies $|f| \rightarrow\left|f^{*}\right|$ and $\|f\|=\left\|f^{*}\right\|$, then the homomorphism $\mathfrak{N} \rightarrow \mathfrak{N}^{*}$ is necessarily an isomorphism and is called an analytical isomorphism; and $\mathfrak{N}$ and $\mathfrak{N}^{*}$ are said to be analytically isomorphic.

There are several elementary theorems which we may mention informally at this point. Thus, we see that the analytical subring generated by a nonvoid subclass $\mathfrak{M}_{0}$ of $\mathfrak{M}$ is the closure of the subring generated by $R$ and $\mathfrak{M}_{0}$. It is obvious from Theorem 75 that an analytical subring contains $|f|$ together with $f$. If $\mathfrak{R}^{*}$ is analytically isomorphic to a closed subring $\mathfrak{N}$, then $\mathfrak{N}^{*}$ is closed. If $\mathfrak{N}^{*}$ is analytically isomorphic to an analytical subring $\mathfrak{N}$, then there exist an analytical subring $\mathfrak{R}^{* *}$ containing $\mathfrak{R}^{*}$ and a function

† See, for instance, Banach, Théorie des Opérations Linéaires, Warsaw, 1932, especially pp. 11, 53; Chittenden, these Transactions, vol. 31 (1929), pp. 290-321. 
$\theta^{*}$ in $\mathfrak{R}^{*}$ which assumes only the values 0 and 1 and does not vanish identically, such that $\mathfrak{R}^{*}$ is the class of all functions $f^{*}=\theta^{*} f^{* *}$ where $f^{* *} \in \mathfrak{N}^{* *}$. To prove this assertion, we first consider the image $R^{*}$ in $\mathfrak{R}^{*}$ of the real field $R$ in $\Re$. If $\alpha \in R$ and $\alpha \geqq 0$, then $\alpha \rightarrow f^{*}$ implies $f^{*} \geqq 0$ : for $\alpha=|\alpha|$ implies $f^{*}=\left|f^{*}\right|$. If $\theta^{*}$ is the correspondent of 1 , then $\theta^{*}=\left(\theta^{*}\right)^{2}$ so that $\theta^{*}$ assumes only the values 0 and 1 . If $f$ is any element in $\mathfrak{R}$, then $f \rightarrow f^{*}$ implies $1 \cdot f \rightarrow \theta^{*} f^{*}$ and hence $f^{*}=\theta^{*} f^{*}$. Since $\mathfrak{N}$ and $\mathfrak{R}^{*}$ are isomorphic it is therefore evident that $\theta^{*}$ does not vanish identically. If $\alpha$ is the rational number $m / n$ and $\alpha \rightarrow f^{*}$, the relation $n \cdot \alpha=m \cdot 1$ or $\alpha+\cdots+\alpha=1+\cdots+1$ implies $n f^{*}=m \theta^{*}$, and hence $f^{*}=m / n \theta^{*}=\alpha \theta^{*}$. If $\alpha$ is arbitrary and $\alpha_{1}$ and $\alpha_{2}$ are rational approximants to $\alpha$ satisfying the inequalities $\alpha_{1} \leqq \alpha \leqq \alpha_{2}$, then $\alpha \rightarrow f^{*}$ implies $\alpha_{1} \theta^{*} \leqq f^{*} \leqq \alpha_{2} \theta^{*}$; and we can therefore conclude that $f^{*}=\alpha \theta^{*}$. We now form the class $\mathfrak{R}^{* *}$ of all functions $f^{* *}=f^{*}+\alpha\left(1-\theta^{*}\right)$ where $f^{*} \epsilon \mathfrak{R}^{*}$ and $\alpha \in R$. Since $\mathfrak{R}^{*}$ is closed and since $f^{*}=\theta^{*} f^{*}$ is in $\mathfrak{N}^{*}$, it is evident that $\mathfrak{R}^{* *}$ is a closed subring of $\mathfrak{M}^{*}$. Moreover, $\mathfrak{R}^{* *}$ contains $R$ : for, if $\alpha$ is any real number, $\alpha \theta^{*}$ is in $\mathfrak{R}^{*}$ and $\alpha=\theta^{*}+\alpha\left(1-\theta^{*}\right)$ is in $\mathfrak{R}^{* *}$. Thus $\mathfrak{R}^{* *}$ is an analytical subring of $\mathfrak{M}^{*}$; and $\mathfrak{R}^{*}$ is obtained from $\mathfrak{R}^{* *}$ in the manner described above. In the space $\Re^{*}$ in which $\mathfrak{M}^{*}$ is defined, the equation $\theta^{*}=1$ defines a closed subspace $\Re^{* *}$. Obviously, $\mathfrak{R}^{*}$ is an analytical subring of the function-ring for $\Re^{* *}$. In order that $\theta^{*}$ be identically equal to 1 or, equivalently, that $\mathfrak{R}^{*}$ be an analytical subring of $\mathfrak{M}^{*}$, it is necessary and sufficient that $\mathfrak{R}^{*}$ contain 1 ; and sufficient that $\Re^{*}$ be connected.

We now establish a fundamental theorem concerning ideals in functionrings.

TheORem 76. A necessary and sufficient condition that an ideal $\mathfrak{A}$ in the function-ring $\mathfrak{M}$ be divisorless is that the quotient-ring $\mathfrak{M} / \mathfrak{A}$ be isomorphic to $R$. Such an ideal is necessarily closed and prime in $\mathfrak{M}$; and the homomorphism $\mathfrak{M} \rightarrow R$ determined by it has the property that $f \rightarrow \alpha$ implies $|f| \rightarrow|\alpha|$ and g.l.b. $f \leqq \alpha \leqq$ l.u.b. $f$.

If $\mathfrak{M} / \mathfrak{A}$ is isomorphic to $R$, the fact that $R$ is a field shows that $R$ has no ideals other than $R$ and $\{0\}$; and it follows that $\mathfrak{A}$ has no ideal divisors other than $\mathfrak{M}$ and $\mathfrak{A}$, in other words, that $\mathfrak{A}$ is divisorless. Since the quotient-ring is a field, $\mathfrak{A}$ is also prime. It is evident that $\mathfrak{A}$ does not contain the unit 1 in $\mathfrak{M}$.

If $\mathfrak{A}$ is a divisorless ideal in $\mathfrak{M}$, the inequality $\mathfrak{A} \neq \mathfrak{M}$ shows that 1 does. not belong to $\mathfrak{A}$. Since the closure $\mathfrak{A}^{-}$is also an ideal, the relation $\mathfrak{A} \subset \mathfrak{A}^{-}$ implies that $\mathfrak{A}=\mathfrak{A}^{-}$or $\mathfrak{M}=\mathfrak{A}-$. The equation $\mathfrak{M}=\mathfrak{A}-$ holds if and only if 1 is in $\mathfrak{A}^{-}$. Now if 1 were an element of $\mathfrak{A}^{-}$, there would exist in $\mathfrak{A}$ a uniformly convergent sequence of functions with 1 as limit. Hence $\mathfrak{A}$ would contain a function $f$ such that g.l.b. $f>0$. Since we would then have $1 / f \in \mathfrak{M}$ and $f \in \mathfrak{Q}$, we 
could conclude that $1=f \cdot(1 / f) \in \mathfrak{A}$, contrary to fact. The equation $\mathfrak{A}=\mathfrak{A}^{-}$is therefore valid; and $\mathfrak{A}$ is closed in $\mathfrak{M}$. We can next show that $\mathfrak{M} / \mathfrak{A}$ is a field. If $f$ is any element in $\mathfrak{M}$, then the ideal generated by $f$ and $\mathfrak{A}$ coincides with $\mathfrak{M}$. Hence there exist functions $g$ and $h$ in $\mathfrak{M}$ and in $\mathfrak{A}$ respectively such that $f g+h=1$. Thus the relation $f \not \equiv 0(\bmod \mathfrak{A})$ implies the existence of a function $g$ in $\mathfrak{M}$ such that $f g \equiv 1(\bmod \mathfrak{A})$, as we wished to prove. It is clear that $\mathfrak{A}$ contains no member of $R$ other than 0 ; and it follows that two members of $R$ are congruent $(\bmod \mathfrak{A})$ if and only if they are identical. Hence the field $\mathfrak{M} / \mathfrak{A}$ contains $R$ as a subfield.

In order to prove that $\mathfrak{M} / \mathfrak{A}$ coincides with its subfield $R$, we shall apply the theory of ordered fields. ${ }^{*}$ The first step is to classify the elements of $\mathfrak{M} / \mathfrak{A}$ as positive, negative, or zero in such a way that $f+g$ and $f g$ are positive, $-f$ negative, when $f$ and $g$ are positive. In this classification we wish to maintain the natural classification of the subfield $R$. For convenience we shall define a similar classification of $\mathfrak{M}$ in such a way that functions congruent $(\bmod \mathfrak{A})$ are assigned to the same class. We say that $f$ is positive if $f \equiv|f|(\bmod \mathfrak{A}), f \not \equiv 0(\bmod \mathfrak{A})$, and that $f$ is negative if $-f$ is positive; and denote the class of positive elements in $\mathfrak{M}$ by $\mathfrak{B}$, the class of negative elements by $\mathfrak{R}$. In order to justify this classification, we prove the following propositions: (1) if $f \in \mathfrak{M}$, then one and only one of the three relations $f \in \mathfrak{P}$, $f \in \mathfrak{N}, f \in \mathfrak{P}$ is valid; (2) if $f \in \mathfrak{M}, g \in \mathfrak{M}, 0 \leqq f \leqq g$, and $g \in \mathfrak{A}$, then $f \in \mathfrak{A}$; (3) if $f \in \mathfrak{P}, g \in \mathfrak{M}$ and $f \equiv g(\bmod \mathfrak{A})$, then $g \in \mathfrak{P} ;(4)$ if $f \in \mathfrak{P}$ and $g \in \mathfrak{P}$, then $f+g \in \mathfrak{P}$ and $f g \in \mathfrak{P}$.

To prove (1) we proceed as follows. Since $(-f+|f|)(f+|f|)=-f^{2}$ $+|f|^{2}=0$, the fact that $\mathfrak{M} / \mathfrak{A}$ is a field implies that at least one of the relations $-f+|f| \equiv 0(\bmod \mathfrak{A})$ and $+f+|f| \equiv 0(\bmod \mathfrak{A})$ is valid. If both hold, then $2 f \equiv(f+|f|)-(-f+|f|) \equiv 0(\bmod \mathfrak{A})$ and therefore $f \equiv 0(\bmod \mathfrak{A})$. We see therefore that one and only one of the three sets of relations $f \equiv|f| \not 0$ $(\bmod \mathfrak{A}),-f \equiv|-f| \not \equiv 0(\bmod \mathfrak{A}), f \equiv 0(\bmod \mathfrak{A})$ is valid, as we wished to show.

We establish (2) by contradiction. Let us suppose that $f \not \equiv 0(\bmod \mathfrak{A})$. Then there exists an element $h$ in $\mathfrak{M}$ such that $f h \equiv 1(\bmod \mathfrak{A})$. By (1) at least one of the relations $-h \equiv|h|(\bmod \mathfrak{H})$ and $h \equiv|h|(\bmod \mathfrak{A})$ is valid. If the first should hold, we would have $1+f|h| \equiv 1-f h \equiv 0(\bmod \mathfrak{A})$, and also $1+f|h| \geqq 1$ on account of the inequality $f \geqq 0$. Here we have a contradiction: it is obvious that $1 /(1+f|h|)$ is in $\mathfrak{M}$; and it follows that $1=(1+f|h|)[1 /(1+f|h|)] \equiv 0(\bmod \mathfrak{A}), 1 \in \mathfrak{A}$. If the second should hold, we would have $1-f|h| \equiv 1-f h \equiv 0(\bmod \mathfrak{A}), g|h|+(1-f|h|) \equiv 0(\bmod \mathfrak{A})$, and also $g|h|+(1-f|h|) \geqq 1$ on account of the inequality $f \leqq g$. Again we have a contradiction. Thus our initial assumption is false, as we wished to prove.

* See van der Waerden, Moderne Algebra, I, Leipzig, 1930, Chapter X. 
We now consider $(3)$. Since $f \equiv g(\bmod \mathfrak{A})$ and $f \not \equiv 0(\bmod \mathfrak{A})$, it is obvious that $g \not \equiv 0(\bmod \mathfrak{A})$. If the relation $-g \equiv|g|(\bmod \mathfrak{A})$ held, we could combine it with $f \equiv|f|(\bmod \mathfrak{A})$ to obtain the relations $|f|+|g| \equiv f-g \equiv 0(\bmod \mathfrak{A})$. We could then conclude by virtue of $(2)$ that $|g| \equiv 0(\bmod \mathfrak{A})$ and hence that $g \equiv g+|g| \equiv 0(\bmod \mathfrak{A})$. This contradiction shows that we must have $g \equiv|g| \not \equiv 0(\bmod \mathfrak{A})$, or $g \in \mathfrak{B}$, in accordance with (1).

The proof of (4) is similar to that of (3). The relations $f \equiv|f| \not \equiv 0(\bmod \mathfrak{A})$ and $g \equiv|g| \not \equiv 0(\bmod \mathfrak{A})$ imply $f g \equiv|f g| \not \equiv 0(\bmod \mathfrak{A}) ;$ in other words, $f \in \mathfrak{P}$ and $g \in \mathfrak{B}$ imply $f g \in \mathfrak{B}$. These relations also imply that $f+g \not \equiv-|f+g|(\bmod \mathfrak{A})$ : for otherwise we would have $|f|+|g|+|f+g| \equiv f+g-(f+g) \equiv 0(\bmod \mathfrak{R})$ and hence, by virtue of $(2),|f| \equiv|g| \equiv 0(\bmod \mathfrak{A})$, contrary to hypothesis. Furthermore, these relations show that $f+g \neq 0(\bmod \mathfrak{A})$ : for otherwise we would have $|f|+|g| \equiv f+g \equiv 0(\bmod \mathfrak{A})$ and hence, by virtue of $(2),|f| \equiv|g| \equiv 0$ $(\bmod \mathfrak{A})$, contrary to hypothesis. It now follows that $f+g \equiv|f+g| \not \equiv 0(\bmod \mathfrak{A})$ or, equivalently, that $f+g \in \mathfrak{B}$, as we wished to prove.

If we remark that the relations $\alpha \equiv|\alpha| \not \equiv 0(\bmod \mathfrak{A})$ and $\alpha=|\alpha| \neq 0$ are equivalent whenever $\alpha \in R$, we see that the partition of $\mathfrak{M}$ into the disjoint classes $\mathfrak{B}, \mathfrak{R}$, and $\mathfrak{A}$ has all the properties required above. It defines a corresponding partition of the field $\mathfrak{M} / \mathfrak{A}$; and this partition of $\mathfrak{M} / \mathfrak{A}$ in turn defines a simple ordering of the field, in a familiar way. ${ }^{*}$ We can now prove that the order so introduced is an archimedean order; in other words, that $f \in \mathfrak{B}$ implies that $n f-1 \epsilon \mathfrak{P}$ for some integer $n$. If the element $f-1 / n$ should belong to $\mathfrak{B}$ for no integer $n$, we would have $-(f-1 / n) \equiv|f-1 / n|(\bmod \mathfrak{A})$ or, equivalently, $(f-1 / n)+|f-1 / n| \epsilon \mathfrak{A}$ for every $n$. On passing to the limit, we would then obtain $f+|f| \epsilon \mathfrak{A}-=\mathfrak{A}$ or, equivalently, $f \mathfrak{A P}$. This contradiction shows that $f-1 / n \in \mathfrak{B}$ for some integer $n$. By (4), we conclude that $n f-1=n(f-1 / n) \in \mathfrak{P}$ for that integer, as we wished. Since the field $\mathfrak{M} / \mathfrak{A}$ has an archimedean ordering which is an extension of the natural ordering of its subfield $R$, it must coincide with $R$.

Finally we consider the special properties of the homomorphism $\mathfrak{M} \rightarrow R$ stated in the theorem. In order that this homomorphism take $f$ into $\alpha$ it is obviously necessary and sufficient that $f \equiv \alpha(\bmod \mathfrak{A})$. If $f \equiv \alpha(\bmod \mathfrak{A})$, the relation $|f-\alpha| \geqq \epsilon>0$ is impossible: for $f \equiv \alpha(\bmod \mathfrak{A})$ implies $f-\alpha \equiv 0$ $(\bmod \mathfrak{A}),|f-\alpha| \equiv 0(\bmod \mathfrak{A}) ;$ and $|f-\alpha| \geqq \epsilon>0$ implies $|f-\alpha| \not \equiv 0(\bmod \mathfrak{A})$ in accordance with (2) above. It follows that g.l.b. $f \leqq \alpha \leqq$ l.u.b. $f$; or, more precisely, that $\alpha$ is a limit point of the range of $f$. The proof that $f \equiv \alpha(\bmod \mathfrak{A})$ implies $|f| \equiv|\alpha|(\bmod \mathfrak{U})$ is simple and will be omitted.

We pass now to the investigation of the connections between $C R$-spaces and $T_{0}$-spaces. The fundamental theorem reads as follows.

\footnotetext{
* See van der Waerden, Moderne Algebra, I, Leipzig, 1930, p. 209.
} 
THEOREM 77. In a $T_{0}$-space $\Re$, let $G$ be the family of all open sets $\mathcal{B}=\Re(\alpha<f<\beta)$ where $f \in \mathfrak{M}$ and $\alpha \in R, \beta \in R$; let $\mathfrak{X}(\mathfrak{r})$ be the intersection of all those sets in $G$ which contain the point $\mathfrak{r}$ in $\Re$; let $X$ be the family of all sets $\mathfrak{X}(\mathfrak{r})$; and let $R^{*}$ be the space obtained by assigning each subset of $X$ specified by the relations $\mathfrak{X}(\mathfrak{r}) \subset \mathcal{B},(\mathfrak{S} \in G$ as a neighborhood of every $\mathfrak{X}(\mathfrak{r})$ which it contains. Then $\Re^{*}$ is a CR-space which is a continuous image of $\Re$ under the correspondence $\mathfrak{r} \rightarrow \mathfrak{X}(\mathfrak{r})$; and the function-rings $\mathfrak{M}$ and $\mathfrak{M}^{*}$ for $\mathfrak{R}$ and $\mathfrak{R}^{*}$ respectively are analytically isomorphic under the correspondence $f \rightarrow f^{*}$ defined by setting $f^{*}(\mathfrak{X}(\mathfrak{r}))=f(\mathfrak{r})$ for each point $\mathfrak{r}$ in $\Re$. In case $\Re$ is itself a CR-space, $\Re^{*}$ is topologically equivalent to $\Re$.

If $\mathfrak{r}$ is an arbitrary point and $f$ an arbitrary function in $\mathfrak{M}$, the set $\Re(\alpha<f<\beta)$ contains $\mathfrak{r}$ if $\alpha$ and $\beta$ are so chosen that $\alpha<f(\mathfrak{r})<\beta$. Hence every point $\mathfrak{r}$ determines a set $\mathfrak{X}(\mathfrak{r})$. It is easily seen that every function in $\mathfrak{M}$ is constant on each set $\mathfrak{X}(\mathfrak{r})$. In fact, if $f(\mathfrak{z}) \neq f(\mathfrak{r}), \alpha$ and $\beta$ could be chosen so that $\Re(\alpha<f<\beta)$ contains $\mathfrak{r}$ and $\mathfrak{X}(\mathfrak{r})$ but not $\mathfrak{z}$. It is therefore clear that $\mathfrak{X}(\mathfrak{r}) \mathfrak{X}(\mathfrak{z}) \neq 0$ implies $\mathfrak{X}(\mathfrak{r})=\mathfrak{X}(\mathfrak{z})$ : for every function in $\mathfrak{M}$ must be constant on $\mathfrak{X}(\mathfrak{r}) \cup \mathfrak{X}(\mathfrak{z})$; and every set $\Re(\alpha<f<\beta), f \in \mathfrak{M}$, must contain both or neither of the sets $\mathfrak{X}(\mathfrak{r})$ and $\mathfrak{X}(\mathfrak{z})$. From these results it is obvious that every set in $G$ is the union of all the sets $\mathfrak{X}(\mathfrak{r})$ which it contains. Hence the assignment of neighborhoods described in the statement of the theorem can be justified through the properties which follow: (1) every $\mathfrak{X}(\mathfrak{r})$ has at least one neighborhood; (2) the intersection of two neighborhoods of $\mathfrak{X}(\mathfrak{r})$ is a neighborhood of $\mathfrak{X}(\mathfrak{r})$; (3) any neighborhood which contains $\mathfrak{X}(\mathfrak{r})$ is a neighborhood of $\mathfrak{X}(\mathfrak{r}) ;(4)$ if $\mathfrak{X}(\mathfrak{r}) \neq \mathfrak{X}(\mathfrak{z})$, there is a neighborhood of $\mathfrak{X}(\mathfrak{r})$ which does not contain $\mathfrak{X}(8)$. The properties (1), (3), (4) are obvious from preceding remarks, while (2) follows at once from the observation $\dagger$ that $\Re(\alpha<f<\beta) \Re(\gamma<g<\delta)$ $=\Re(0<h<\lambda)$ where $h=\min [(f-\alpha)(\beta-f),(g-\gamma)(\delta-g)], \lambda>\|h\|$. Accordingly the introduction of the indicated neighborhood-system yields a $T_{1}$-space $\Re^{*}$. Since the sets $\mathfrak{X}(\mathfrak{r})$ are disjoint, the correspondence $\mathfrak{r} \rightarrow \mathfrak{X}(\mathfrak{r})$ from $\Re$ to $\Re^{*}$ is univocal. The open sets specified in $\Re^{*}$ by the relations $\mathfrak{X}(\mathfrak{r}) \subset \mathfrak{B}, \mathfrak{B}_{\epsilon} \in \mathcal{G}$ constitute a basis for $\Re^{*}$ and have as antecedents in $\Re$ the corresponding sets $\mathcal{H}$ in $G$. The correspondence $\mathfrak{r} \rightarrow \mathfrak{X}(\mathfrak{r})$ is therefore continuous. Furthermore, if $\Re$ is a $C R$-space, the family $G$ is a basis for $\Re$, so that $\mathfrak{X}(\mathfrak{r})=\{\mathfrak{r}\}$ and the correspondence $\mathfrak{r} \rightarrow \mathfrak{X}(\mathfrak{r})$ is biunivocal and bicontinuous. Thus $\Re^{*}$ is a continuous image of $\Re$, and is topologically equivalent to $\Re$ when $\Re$ is a $C R$-space. If $f \in \mathfrak{M}$, the function $f^{*}$ defined in $\Re^{*}$ by putting $f^{*}(\mathfrak{X}(\mathfrak{r}))=f(\mathfrak{r})$ is bounded, single-valued, and real. We see also that $f^{*}$ is continuous since the set

$\dagger$ We use the relations $2 \max (f, g)=|f-g|+f+g, \quad 2 \min (f, g)=-|f-g|+f+g$ here and elsewhere in this section, when properties of $\max (f, g)$ or $\min (f, g)$ are needed. 
$\Re^{*}\left(\alpha<f^{*}<\beta\right)$ is the open set of all $\mathfrak{X}(\mathfrak{r})$ such that $\mathfrak{X}(\mathfrak{r}) \subset \Re(\alpha<f<\beta)$. Thus $f^{*}$ is in $\mathfrak{M}^{*}$. On the other hand if $f^{*}$ is any function in $\mathfrak{M}^{*}$, the function $f(\mathfrak{r})=f^{*}(\mathfrak{X}(\mathfrak{r}))$ is a bounded real function; and $f(\mathfrak{r})$ is also continuous since it defines a correspondence $\mathfrak{r} \rightarrow f(\mathfrak{r})$ from $\Re$ to $R$ which is obtained by eliminating $\mathfrak{X}(\mathfrak{r})$ from the continuous correspondences $\mathfrak{r} \rightarrow \mathfrak{X}(\mathfrak{r}), \mathfrak{X}(\mathfrak{r}) \rightarrow f^{*}(\mathfrak{X}(\mathfrak{r}))$ carrying $\Re$ into $\Re^{*}$ and $\Re^{*}$ into $R$ respectively. Thus the correspondence $f \rightarrow f^{*}$ takes $\mathfrak{M}$ univocally into $\mathfrak{M}^{*}$. It is easily verified that this correspondence is an analytical isomorphism in accordance with Definition 22. Finally, it is evident that $\Re^{*}$ is a $C R$-space, since the sets $\Re^{*}\left(\alpha<f^{*}<\beta\right)$ constitute a basis for $\Re^{*}$ by virtue of the characterization given for them above. We may note in passing that $\mathfrak{X}(\mathfrak{r})$ can obviously be obtained as the intersection of the closed sets $\Re(f=f(\mathfrak{r})), f \in \mathfrak{M}$, and is therefore closed.

The immediate significance of Theorem 77 is seen to be that in studying function-rings we may restrict attention to the case of $C R$-spaces without any loss of generality.

We shall now obtain some information concerning the connections between function-rings and algebraic maps for $C R$-spaces.

THEOREM 78. In a $C R$-space $\Re$, let $G$ be the basis of all sets $\Re(\alpha<f<\beta)$ where $f$ is in the function-ring $\mathfrak{M}$; let $A$ be the basic ring generated by $G$; let $\mathfrak{A}$ be $a$ divisorless ideal in $\mathfrak{M}$; and let $\mathfrak{a}(\mathfrak{H})$ be the ideal in $A$ determined as the class of all sets $a$ in $A$ such that, for at least one choice of $f, \alpha$, and $\beta$, the relations $a<\Re(\alpha<f<\beta), f \equiv \gamma(\bmod \mathfrak{A})$, and either $\gamma<\alpha$ or $\gamma>\beta$ are valid. Then in the bicompact Boolean space $\mathbb{E}(A)$, the closed sets $\mathfrak{Z}(\mathfrak{U})=\mathbb{F}^{\prime}(\mathfrak{a}(\mathfrak{A})$ ) are disjoint and constitute a continuous covering family $Z$. Under the usual topology, Z defines a bicompact $H$-space $\mathfrak{Q}$.

We must first show that $\mathfrak{a}(\mathfrak{H})$ is an ideal in $A$. It is evident that $0 \in \mathfrak{a}(\mathfrak{A})$ and that $a \in \mathfrak{a}(\mathfrak{H})$ and $a>c$ imply $c \in \mathfrak{a}(\mathfrak{H})$. Hence we have only to prove that $a \in \mathfrak{a}(\mathfrak{A})$ and $b \in \mathfrak{a}(\mathfrak{H})$ imply $a \vee b \in \mathfrak{a}(\mathfrak{H})$. By hypothesis, we have $a<\mathfrak{R}(\alpha<f<\beta)$ and $b<\mathfrak{R}(\gamma<g<\delta)$ where $f \equiv \sigma(\bmod \mathfrak{A}), g \equiv \tau(\bmod \mathfrak{A})$, and $\sigma$ and $\tau$ lie respectively outside the closed intervals $[\alpha, \beta]$ and $[\gamma, \delta]$. If we put $h=|f-\sigma|+|g-\tau|$, $\epsilon>0, \eta>\|h\|$, and choose $\epsilon$ sufficiently small, it is seen that $a \vee b<\Re(h>\epsilon)$ $=\mathfrak{R}(\epsilon<h<\eta), h \equiv 0(\bmod \mathfrak{A})$ and hence that $a \vee b \in \mathfrak{a}(\mathfrak{A})$, as we wished to prove.

Next we show that $\mathfrak{A}_{1} \neq \mathfrak{A}_{2}$ implies $\mathfrak{a}\left(\mathfrak{A}_{1}\right) \vee \mathfrak{a}\left(\mathfrak{H}_{2}\right)=\mathfrak{e}$ and hence $\mathfrak{B}\left(\mathfrak{H}_{1}\right) \mathfrak{B}\left(\mathfrak{A}_{2}\right)$ $=\mathfrak{E}^{\prime}\left(\mathfrak{a}\left(\mathfrak{A}_{1}\right)\right) \mathfrak{F}^{\prime}\left(\mathfrak{a}\left(\mathfrak{A}_{2}\right)\right)=\mathfrak{F}^{\prime}\left(\mathfrak{a}\left(\mathfrak{A}_{1}\right) \vee \mathfrak{a}\left(\mathfrak{H}_{2}\right)\right)=\mathfrak{F}^{\prime}(\mathfrak{e})=0$. If $\mathfrak{A}_{1} \neq \mathfrak{A}_{2}$, the ideal generated in $\mathfrak{M}$ by $\mathfrak{A}_{1}$ and $\mathfrak{A}_{2}$ coincides with $\mathfrak{M}$; in other words, there exist functions $f$ and $g$ such that $f+g=1, f \in \mathfrak{A}_{1}, g \in \mathfrak{A}_{2}$. The relations $|f| \equiv 0\left(\bmod \mathfrak{A}_{1}\right)$, $|g| \equiv 0\left(\bmod \mathfrak{A}_{2}\right)$ show that the sets $a=\Re(|f|>1 / 3)$ and $b=\Re(|g|>1 / 3)$ belong to $\mathfrak{a}\left(\mathfrak{A}_{1}\right)$ and to $\mathfrak{a}\left(\mathfrak{A}_{2}\right)$ respectively. Since $1=f+g \leqq|f|+|g|$, we must 
have $a^{\prime} b^{\prime}=\Re(|f| \leqq 1 / 3) \cdot \Re(|g| \leqq 1 / 3)=0$ or, equivalently, $a \vee b=e$. It follows that $\mathfrak{a}\left(\mathfrak{A}_{1}\right) \vee \mathfrak{a}\left(\mathfrak{A}_{2}\right)=e$, as we wished to prove.

We must also show that; if $p$ is any prime ideal in $A$, there exists a divisorless ideal $\mathfrak{A}$ in $\mathfrak{M}$ for which the equivalent relations $\mathfrak{p} \in \mathfrak{Z}(\mathfrak{A})$, $\mathfrak{p} \mathfrak{E}(\mathfrak{a}(\mathfrak{A}))$, $\mathfrak{a}(\mathfrak{A}) \subset \mathfrak{p}$ are valid. By Theorem 76 , the construction of a divisorless ideal is equivalent to the construction of a homomorphism $\mathfrak{M} \rightarrow R$. Now, if $f$ is a fixed function in $\mathfrak{M}$, we may classify the open intervals $(\alpha, \beta),-\infty<\alpha<\beta<+\infty$, according to the relation of the set $\Re(\alpha<f<\beta)$ to the given prime ideal $p$ in $A$; in particular, we consider the intervals $(\alpha, \beta)$ for which $\Re(\alpha<f<\beta) \in \mathfrak{p}$. We prove that there is exactly one real number which belongs to none of the latter intervals. First let us show that there is at least one. If there were not, the open intervals $(\alpha, \beta)$ under consideration would cover the closed interval $\left[\alpha^{\prime}, \beta^{\prime}\right]$ where $\alpha^{\prime}$ and $\beta^{\prime}$ are bounds for $f$. By the Heine-Borel-Lebesgue covering property, a finite number of the intervals $(\alpha, \beta)$ would suffice to cover $\left[\alpha^{\prime}, \beta^{\prime}\right]$; and in consequence a finite number of the sets $\Re(\alpha<f<\beta)$ in $\mathfrak{p}$ would suffice to cover $\Re$. Here we have a contradiction, since no finite union of sets belonging to $\mathfrak{p}$ can coincide with the unit $\Re$ in $A$. Thus there exists at least one real number $\gamma$ of the desired kind; and it is evident that any such number $\gamma$ must satisfy the inequality g.l.b. $f \leqq \gamma \leqq l$.u.b. $f$ or, more precisely, must belong to the closure of the range of $f$. Now let us prove that, if $\gamma_{1}$ and $\gamma_{2}$ are such numbers, then $\gamma_{1}=\gamma_{2}$. By hypothesis, the sets $\Re\left(\gamma_{1}-\epsilon<f<\gamma_{1}+\epsilon\right)$ and $\Re\left(\gamma_{2}-\epsilon<f<\gamma_{2}+\epsilon\right)$ belong to $\mathfrak{p}$ for no positive $\epsilon$. It follows that these sets must have non-void intersection for every positive $\epsilon$ and hence that the open intervals $\left(\gamma_{1}-\epsilon, \gamma_{1}+\epsilon\right)$ and $\left(\gamma_{2}-\epsilon, \gamma_{2}+\epsilon\right)$ must have points in common for every positive $\epsilon$. Thus we conclude that $\gamma_{1}=\gamma_{2}$. In view of the preceding construction, we see that the prime ideal $\mathfrak{p}$ assigns to each $f$ in $\mathfrak{M}$ a unique corresponding real number $\gamma$. We wish now to prove that the correspondence $f \rightarrow \gamma$ defines a homomorphism $\mathfrak{M} \rightarrow R$. By construction, it is evident that $f \rightarrow \gamma$ implies $f-\gamma \rightarrow 0$. It is also evident that, if $f$ is constant, then $f \rightarrow \gamma$ if and only if $f=\gamma$. If we can establish the propositions (1) $f \rightarrow 0, g \rightarrow 0$ imply $f+g \rightarrow 0$, and (2) $f \rightarrow 0$ implies $f g \rightarrow 0$, we can then show that $f \rightarrow \gamma, g \rightarrow \delta$ imply $(f-\gamma)+(g-\delta) \rightarrow 0$ or, equivalently, $f+g \rightarrow \gamma+\delta$ and $(f-\gamma) g+\gamma(g-\delta) \rightarrow 0$ or, equivalently, $f g \rightarrow \gamma \delta$. If $f \rightarrow 0$ and $g \rightarrow 0$, the sets $\Re(|f|<\epsilon / 2)$ and $\Re(|g|<\epsilon / 2)$ belong to $\mathfrak{p}$ for no positive $\epsilon$; and their intersection likewise belongs to $\mathfrak{p}$ for no positive $\epsilon$. Since $\Re(|f+g|<\epsilon) \supset \Re(|f|<\epsilon / 2) \Re(|g|<\epsilon / 2)$, the set $\Re(|f+g|<\epsilon)$ belongs to $\mathfrak{p}$ for no positive $\epsilon$. It follows that $f+g \rightarrow 0$. Similarly if $f \rightarrow 0$, the set $\Re(|f g|<\epsilon)$ contains the set $\Re(|f|<\epsilon /\|g\|)$ which does not belong to $p$ for any positive $\epsilon$; and we conclude that $f g \rightarrow 0$. Thus the correspondence $f \rightarrow \gamma$ is a homomorphism.

If $\mathfrak{A}$ is the ideal determined by this homomorphism, we must show that 
$\mathfrak{a}(\mathfrak{A}) \subset \mathfrak{p}$. If $a \in \mathfrak{a}(\mathfrak{A})$, there exists a function $f$ and real numbers $\alpha$ and $\beta$ such that $a<\Re(\alpha<f<\beta)$ and $f \rightarrow \gamma$, where $\gamma$ is outside the closed interval $[\alpha, \beta]$. For sufficiently small positive $\epsilon$ we have $\Re(\gamma-\epsilon<f<\gamma+\epsilon) \Re(\alpha<f<\beta)=0$. Since, by our construction of $\gamma$, the set $\Re(\gamma-\epsilon<f<\gamma+\epsilon)$ does not belong to $\mathfrak{p}$, we must therefore have $\Re(\alpha<f<\beta) \in \mathfrak{p}$. It follows that $a \in \mathfrak{p}$ and that $\mathfrak{a}(\mathfrak{U}) \subset \mathfrak{p}$.

The family $Z$ is now seen to cover $\mathbb{E}(A)$; but we wish to show further that $Z$ is continuous. If $a$ is any element in $A$ such that $\mathbb{E}(a) \supset Z(\mathfrak{A})$, we have to find an element $b$ in $A$ such that $\mathfrak{Z}(\mathfrak{H}) \subset \mathbb{E}(b)$ and such that $\mathfrak{Z}(\mathfrak{B}) \mathscr{E}^{\prime}(a) \neq 0$ implies $\mathfrak{Z}(\mathfrak{B}) \mathfrak{E}(b)=0$. The relations $\mathbb{E}(a) \supset \mathfrak{Z}(\mathfrak{\mathfrak { A }}), \mathbb{E}\left(a^{\prime}\right) \subset \mathfrak{E}(\mathfrak{a}(\mathfrak{A}))$, and $a^{\prime} \in \mathfrak{a}(\mathfrak{A})$ are equivalent. Hence we can find $f, \alpha$, and $\beta$ so that $a^{\prime}<\Re(\alpha<f<\beta)$ where $f \rightarrow \gamma$ and $\gamma$ is outside the closed interval $[\alpha, \beta]$; and we then see that $a>\Re(|g| \leqq \epsilon)$ where $g=f-\gamma$ and $\epsilon$ is a sufficiently small positive number. We now choose $b$ as the set $\Re(|g| \leqq \epsilon / 3)$. Since $b^{\prime}=\Re(|g|>\epsilon / 3)$ where $|g| \equiv 0(\bmod \mathfrak{A})$, we see that $b^{\prime} \mathfrak{\epsilon a}(\mathfrak{A})$ and hence that $\mathfrak{E}(b) \supset \mathfrak{Z}(\mathfrak{A})$. If $\mathfrak{B}$ is a divisorless ideal in $\mathfrak{M}$ such that $\mathfrak{Z}(\mathfrak{B}) \mathfrak{E}\left(a^{\prime}\right)=\mathfrak{Z}(\mathfrak{B}) \mathbb{E}^{\prime}(a) \neq 0$ and if the homomorphism $\mathfrak{M} \rightarrow \mathfrak{M} / \mathfrak{B}$ carries $g$ into $\delta$, we introduce the set $c=\Re(|g-\delta| \leqq \epsilon / 3)$ in $A$. Since $c^{\prime}=\Re(|g-\delta|$ $>\epsilon / 3)$ where $|g-\delta| \equiv 0(\bmod \mathfrak{B})$, we have $c^{\prime} \epsilon \mathfrak{a}(\mathfrak{B})$, $\mathbb{E}(c) \supset \mathfrak{Z}(\mathfrak{B})$. The relations $\mathbb{E}\left(a^{\prime} c\right)=\mathbb{E}^{\prime}(a) \mathfrak{E}(c) \supset \mathbb{E}^{\prime}(a) \mathfrak{Z}(\mathfrak{B}) \neq 0$ show that $a^{\prime} c \neq 0$. From the relations $\Re(|g|>\epsilon) \Re(|g-\delta| \leqq \epsilon / 3) \supset a^{\prime} c \neq 0$, we infer that $|\delta|>2 \epsilon / 3$. We then conclude that $b c=\Re(|g| \leqq \epsilon / 3) \Re(|g-\delta| \leqq \epsilon / 3)=0$ and hence that $\mathbb{E}(b) \mathfrak{Z}(\mathfrak{B})$ c $\mathfrak{E}(b) \mathfrak{E}(c)=\mathfrak{E}(b c)=\mathfrak{F}(0)=0, \mathfrak{E}(b) \mathfrak{Z}(\mathfrak{B})=0$, as we wished to prove.

If we now impose the usual topology upon the family $Z$, we obtain an $H$-space $\mathfrak{Q}$ and a map $m(\mathfrak{Q}, \mathfrak{E}(A), Z)$ in accordance with Theorem 23. Since $Z$ is a continuous covering family, Theorem 22 shows that $\mathfrak{Q}$ is a continuous image of $\mathbb{E}(A)$. It follows that $\mathfrak{Q}$ is a bicompact $H$-space.

Theorem 79. The space $\mathfrak{Q}$ of Theorem 78 is an immediate, and hence strict, $H$-extension of the given $C R$-space $\Re$. Every function in $\mathfrak{M}$ can be extended from $\Re$ to $\mathfrak{Q}$ so as to be continuous, and hence bounded, in $\mathfrak{Q}$. If $f \in \mathfrak{M}$ and $f^{*}$ is its extension to $\mathfrak{Q}$, then the correspondence $f \rightarrow f^{*}$ is an analytical isomorphism between the function-rings for $\Re$ and $\mathfrak{Q}$. In case $\Re$ is a bicompact $H$-space, $\mathfrak{Q}$ coincides with $\Re$.

Considering the algebraic map $m(\Re, \mathbb{E}(A), X)$, where $A$ is the basic ring described in Theorem 78 , we show that each set $\mathfrak{X}(\mathfrak{r})$ coincides with a suitable member of the family $Z$ of that theorem. If $r$ is an arbitrary point in $\Re$, the correspondence $f \rightarrow f(\mathfrak{r})$ defines a homomorphism $\mathfrak{M} \rightarrow R$. The associated divisorless ideal $\mathfrak{A}=\mathfrak{A}(\mathfrak{r})$ then has the property that $\mathfrak{X}(\mathfrak{r})=\dot{\mathfrak{Z}}(\mathfrak{A}(\mathfrak{r}))$. We prove this statement as follows. If $a \in A$ and $\mathfrak{E}(a) \supset \mathfrak{X}(\mathfrak{r})$, then the basis $G$ contains a set $b=\Re(|f|<\epsilon), f(\mathfrak{r})=0$, which contains $\mathfrak{r}$ and is contained in $a$ : for Theorem 28 shows that $\mathrm{r}$ is interior to $a$. The relations $b^{\prime}=\Re(|f| \geqq \epsilon) \subset \Re(|f|>\epsilon / 2)$ and 
$f \equiv 0(\bmod \mathfrak{A}(\mathfrak{r}))$ show that $b^{\prime} \in \mathfrak{a}(\mathfrak{A}(\mathfrak{r}))$ or, equivalently, that $\mathfrak{E}(b) \supset \mathfrak{Z}(\mathfrak{A}(\mathfrak{r}))$. Since $\mathbb{E}(a) \supset \mathbb{E}(b)$, we conclude that $\mathbb{E}(a)$ also contains $\mathbb{Z}(\mathfrak{A}(\mathfrak{r}))$. On the other hand, if $a \epsilon A$ and $\mathfrak{E}(a) \supset \mathfrak{Z}(\mathfrak{A}(\mathfrak{r}))$, we know that $a^{\prime} \mathfrak{\in a}(\mathfrak{A}(\mathfrak{r}))$. Hence there exist a function $f$ and a positive number $\epsilon$ such that $a^{\prime}<\Re(|f|>\epsilon)$ where $f \equiv 0(\bmod \mathfrak{A}(\mathfrak{r}))$ or, equivalently, $f(\mathfrak{r})=0$. Consequently the open set $b=\Re(|f|<\epsilon)$ belongs to $A$, contains $\mathfrak{r}$, and is contained in $a$. Since $\mathfrak{r}$ is thus interior to $a$, we conclude that $\mathfrak{E}(a) \supset \mathfrak{X}(\mathfrak{r})$. The equivalence of the relations $\mathfrak{E}(a) \supset \mathfrak{X}(\mathfrak{r})$, $\mathfrak{E}(a) \supset \mathfrak{Z}(\mathfrak{A}(\mathfrak{r}))$ implies that $\mathfrak{X}(\mathfrak{r})=\mathfrak{Z}(\mathfrak{A}(\mathfrak{r}))$, as we wished to show. Theorem 41 now shows that $\mathfrak{Q}$ is an immediate $H$-extension of $\Re$; and Theorems 64 and 75 show that $\mathfrak{Q}$ must be a strict extension of $\Re$.

In discussing the extension of functions from $\Re$ to $\mathfrak{Q}$ we may regard $\Re$ as a subspace of $\mathfrak{Q}$ and we may even identify $\Re$ and $\mathfrak{Q}$ with the topologized families $X$ and $Z$ respectively. If $f \in \mathfrak{M}$, we define the extended function $f^{*}$ by putting $f^{*}(\mathfrak{Z}(\mathfrak{A}))=\boldsymbol{\gamma}$ where $f \rightarrow \gamma$ under the homomorphism $\mathfrak{M} \rightarrow \mathfrak{M} / \mathfrak{A}$. That $f^{*}$ is actually an extension of $f$ appears at once from the relations $f^{*}(\mathfrak{X}(\mathfrak{r}))=f^{*}(\mathfrak{Z}(\mathfrak{A}(\mathfrak{r})))=f(\mathfrak{r})$. We see also that $f^{*}=g^{*}$ if and only if $f=g$, and that $(f+g)^{*}=f^{*}+g^{*},(f g)^{*}=f^{*} g^{*},|f|^{*}=\left|f^{*}\right|$. It is evident that $\|f\| \leqq\left\|f^{*}\right\|$; and we know that $f \rightarrow \gamma$ implies $|\gamma| \leqq\|f\|$. Hence we see that $\|f\|=\left\|f^{*}\right\|$. To show that $f^{*}$ is continuous, we have to prove that $\mathfrak{Q}\left(\alpha<f^{*}<\beta\right)$ is an open set. If $\mathfrak{Z}\left(\mathfrak{A}_{0}\right)$ represents a point $\mathfrak{q}_{0}$ in this set, then $f^{*}\left(\mathfrak{B}\left(\mathfrak{A}_{0}\right)\right)=\gamma_{0}$ where $f \rightarrow \gamma_{0}$ under the homomorphism $\mathfrak{M} \rightarrow \mathfrak{M} / \mathfrak{A}_{0}$ and $\gamma_{0}$ satisfies the inequality $\alpha<\gamma_{0}<\beta$. Putting $0<\epsilon<\min \left(\gamma_{0}-\alpha, \beta-\gamma_{0}\right)$ we consider the set $a=\Re\left(\gamma_{0}-\epsilon<f<\gamma_{0}+\epsilon\right)$ $=\Re\left(\left|f-\gamma_{0}\right|<\epsilon\right)$ in $A$. We shall show that the open set specified in $\mathfrak{Q}$ by the relation $\mathfrak{Z}(\mathfrak{H}) \subset \mathbb{E}(a)$ contains $\mathfrak{q}_{0}$ and is contained in $\mathfrak{Q}\left(\alpha<f^{*}<\beta\right)$; and we can then infer that $\mathfrak{Q}\left(\alpha<f^{*}<\beta\right)$ is open. First we prove that $\mathfrak{Z}\left(\mathfrak{A}_{0}\right) \subset \mathbb{E}(a)$. The set $b=\Re\left(\left|f-\gamma_{0}\right|>\epsilon / 2\right)$ is in $A$ and obviously contains $a^{\prime}=\Re\left(\left|f-\gamma_{0}\right| \geqq \epsilon\right)$. Since $f-\gamma_{0} \equiv 0\left(\bmod \mathfrak{A}_{0}\right)$, we see that $b \in \mathfrak{a}\left(\mathfrak{A}_{0}\right)$ and hence that $a^{\prime} \in \mathfrak{a}\left(\mathfrak{A}_{0}\right)$. It follows that $\mathbb{E}(a) \supset \mathfrak{Z}\left(\mathfrak{H}_{0}\right)$, as we wished to show. Secondly, we prove that $\mathfrak{Z}(\mathfrak{A}) \subset \mathbb{E}(a)$ implies $f^{*}(\mathfrak{Z}(\mathfrak{A}))=\gamma$ where $\alpha<\gamma<\beta$; and we can then infer that the point $q$ represented by $\mathfrak{Z}(\mathfrak{H})$ is in $\mathfrak{Q}\left(\alpha<f^{*}<\beta\right)$. Now $\mathfrak{Z}(\mathfrak{A}) \subset \mathfrak{E}(a)$ implies that $\mathfrak{R}\left(\left|f-\gamma_{0}\right| \geqq \epsilon\right)=a^{\prime} \boldsymbol{\epsilon a}(\mathfrak{H})$. Hence, if $f \rightarrow \gamma$ under the homomorphism $\mathfrak{M} \rightarrow \mathfrak{M} / \mathfrak{A}$, we must have $\left|\gamma-\gamma_{0}\right| \leqq \epsilon$ : for the relations $a \vee a^{\prime}=e, a^{\prime} \in \mathfrak{a}(\mathfrak{H})$, and $\mathfrak{a}(\mathfrak{A}) \neq \mathrm{e}$ show that $a$ is not in $\mathfrak{a}(\mathfrak{A})$ and hence that $\gamma-\gamma_{0}$ is not outside the closed interval $[-\epsilon, \epsilon]$. Since $f \rightarrow \gamma$ implies $f^{*}(\mathfrak{Z}(\mathfrak{H}))=\gamma$ and since $\alpha<\gamma_{0}-\epsilon \leqq \gamma$ $\leqq \gamma_{0}+\epsilon<\beta$, our proof is brought to the desired conclusion.

If an arbitrary bounded continuous real function $f^{*}$ in $\mathfrak{Q}$ is restricted to the subspace $\Re$, the restricted or partial function $f$ obviously belongs to $\mathfrak{M}$. Since $\Re$ is everywhere dense in $\mathfrak{Q}$, the extension of $f$ to $\mathfrak{Q}$ must be the original function $f^{*}$. We conclude therefore that the correspondence $f \rightarrow f^{*}$ defines an analytical isomorphism of the function-rings $\mathfrak{M}$ and $\mathfrak{M}^{*}$ for $\mathfrak{R}$ and $\mathfrak{Q}$ respectively. 
If the space $\Re$ is a bicompact $H$-space, it is normal and hence completely regular. The corresponding space $\mathfrak{Q}$ must then coincide with $\Re$, since a bicompact $H$-space is absolutely closed with respect to immediate or strict $H$-extension.

Obviously Theorem 79 provides a further reduction of the theory of function-rings: it enables us to restrict attention to the case where the underlying $T_{0}$-space $\Re$ is a bicompact $H$-space. We shall continue the analysis of function-rings under this assumption. First we state a result which is a direct corollary of Theorem 79.

THEOREM 80. If $\mathfrak{A}$ is a divisorless ideal in the function-ring for a bicompact $H$-space $\Re$, then there exists a point $\mathfrak{r}$ in $\Re$ such that $f \in \mathfrak{A}$ if and only if $f(\mathfrak{r})=0$; the homomorphism $\mathfrak{M} \rightarrow \mathfrak{M} / \mathfrak{A}$ carries $f$ into $f(\mathfrak{r})$.

The proof of Theorem 79 shows that the families $X$ and $Z$ coincide when $\Re$ is a bicompact $H$-space; and the result stated here then follows from the relation $\mathfrak{X}(\mathfrak{r})=\mathfrak{Z}(\mathfrak{H}(\mathfrak{r}))$ previously noted. A direct proof can also be given. Since $\Re$ is bicompact, the range of any function $f$ in $\mathfrak{M}$ is a bounded closed set of real numbers. Hence $f \in \mathfrak{A}$ implies that $f \rightarrow 0$ under the homomorphism $\mathfrak{M} \rightarrow \mathfrak{M} / \mathfrak{A}$ and that the set $\mathfrak{R}(f=0)$ is non-void. If $f_{1}, \cdots, f_{n}$ are in $\mathfrak{A}$ we therefore have $\Re\left(f_{1}=0\right) \cdots \Re\left(f_{n}=0\right)=\Re\left(\left|f_{1}\right|=0\right) \cdots \Re\left(\left|f_{n}\right|=0\right)=\left(\Re\left|f_{1}\right|+\cdots\right.$ $\left.+\left|f_{n}\right|=0\right) \neq 0$, since $\left|f_{1}\right|+\cdots+\left|f_{n}\right| \in \mathfrak{A}$. We see therefore that, because of the bicompactness of $\Re$, the intersection of all the closed sets $\Re(f=0)$, where $f \in \mathfrak{A}$, is non-void. It is now evident that the divisorless ideal $\mathfrak{A}(\mathfrak{r})$ defined by the homomorphic correspondence $f \rightarrow f(\mathfrak{r})$, where $\mathfrak{r}$ is a point of this intersection, contains $\mathfrak{A}$. Hence $\mathfrak{A}=\mathfrak{A}(\mathfrak{r})$, as we wished to show.

We pass now to a study of subrings of $\mathfrak{M}$.

THEOREM 81. If $\mathfrak{M}$ is the function-ring for a bicompact $H$-space $\Re$; if $\mathfrak{R}$ is an arbitrary non-void subclass of $\mathfrak{M}$; if $G_{\mathfrak{N}}$ is the family of open sets $\Re(\alpha<f<\beta)$, $f \in \mathfrak{N} ;$ if $\mathfrak{X}_{\mathfrak{N}}(\mathfrak{r})$ is the intersection of all sets $\mathfrak{B}_{\mathfrak{R}}$ in $G_{\mathfrak{N}}$ which contain the point $\mathfrak{r}$ in $\Re$; if $X_{\mathfrak{R}}$ is the family of all sets $\mathfrak{X}_{\mathfrak{R}}(\mathfrak{r}), \mathfrak{r} \in \Re$; and if $\Re_{\mathfrak{R}}$ is the space obtained by assigning each subset of $X_{\mathfrak{N}}$ specified by a relation $\mathfrak{X}_{\mathfrak{N}}(\mathfrak{r}) \subset \mathfrak{W}_{\mathfrak{N}}$, where $\mathfrak{S}_{\mathfrak{N}}=\mathfrak{G}_{\mathfrak{N}}^{(1)} \ldots \mathfrak{G}_{\mathfrak{N}}^{(n)}$ and $\mathfrak{S}_{\mathfrak{N}}^{(k)} \epsilon G_{\Re}$ for $k=1, \cdots, n$, as a neighborhood of every $\mathfrak{X}_{\mathfrak{N}}(\mathfrak{r})$ which it contains; - then $\Re_{\mathfrak{N}}$ is a bicompact $H$-space which is a continuous image of $\Re$. If $\Re_{1} \supset \Re_{2}$, then $\Re_{\Re_{2}}$ is a continuous image of $\Re_{\Re_{1}}$; and $\Re_{\mathfrak{R}}$ is topologically equivalent to $\mathfrak{R}$. If $\mathfrak{R}^{*}$ is the analytical subring of $\mathfrak{M}$ generated by $\Re$, then $\Re_{\Re^{*}}$ is topologically equivalent to $\Re_{\mathfrak{N}}$.

The argument developed in the proof of Theorem 77 can be applied here without essential modifications. Thus we find that the sets $\mathfrak{X}_{\mathfrak{N}}(\mathfrak{r})$ are disjoint and cover $\Re$; that every function in $\mathfrak{R}$ is constant on each set $\mathfrak{X}_{\mathfrak{N}}(\mathfrak{r})$; that every set in $G_{\mathfrak{N}}$ is the union of the sets $\mathfrak{X}_{\mathfrak{N}}(\mathfrak{r})$ which it contains; and that 
$\mathfrak{X}_{\mathfrak{R}}(\mathfrak{r})$, as the intersection of all the closed sets $\Re(f=f(\mathfrak{r}))$ where $f \in \Re$, is itself a closed set. Moreover, we see that, if $\mathcal{G}_{\mathfrak{R}}^{(k)}=\Re\left(\alpha_{k}<f_{k}<\beta_{k}\right), f_{k} \in \mathfrak{N}$, for $k=1, \cdots, n$, then the intersection $\mathfrak{W}_{\mathfrak{N}}=\mathfrak{G}_{\mathfrak{N}}^{(1)} \ldots \mathcal{G}_{\mathfrak{N}}^{(n)}$ is the union of all the sets $\mathfrak{X}_{\mathfrak{R}}(\mathfrak{r})$ which it contains: for $\mathfrak{r} \in \mathfrak{W}_{\mathfrak{N}}$ implies $\alpha_{k}<f_{k}(\mathfrak{r})<\beta_{k}$ for $k=1, \cdots, n$ and hence $\mathfrak{X}_{\mathfrak{N}}(\mathfrak{r}) \subset \mathfrak{S}_{\mathfrak{N}}{ }^{(k)}$ for $k=1, \cdots, n$. Thus the neighborhood-system described in the theorem can be imposed upon the family $\mathcal{X}_{\mathfrak{n}}$; and the resulting space $\Re_{\Re}$ is a $T_{1}$-space. As in the proof of Theorem 77, we see next that the correspondence $\mathfrak{r} \rightarrow \mathfrak{X}_{\mathfrak{R}}(\mathfrak{r})$ from $\Re$ to $\Re_{\mathfrak{R}}$ is univocal and continuous; and that it carries each function $f$ in $\Re$ into a bounded continuous function $f^{*}$ in $\Re_{\mathfrak{N}}$ given by $f^{*}\left(\mathfrak{X}_{\mathfrak{N}}(\mathfrak{r})\right)=f(\mathfrak{r})$. It is now easily seen that $\Re_{\mathfrak{R}}$ is a $C R$-space: for the open set in $\Re_{\mathfrak{R}}$ specified by the relation $\mathfrak{X}_{\mathfrak{R}}(\mathfrak{r}) \subset \mathfrak{S}_{\mathfrak{N}}$, where $\mathfrak{W}_{\mathfrak{R}}=\Re\left(\alpha_{1}<f_{1}<\beta_{1}\right)$ $\cdots \Re\left(\alpha_{n}<f_{n}<\beta_{n}\right)$ and $f_{k} \in \mathfrak{R}$ for $k=1, \cdots, n$, coincides with the set

$$
\Re_{\mathfrak{R}}\left(\alpha_{1}<f_{1}^{*}<\beta_{1}\right) \cdots \Re_{\mathfrak{R}}\left(\alpha_{n}<f_{n}^{*}<\beta_{n}\right)=\Re_{\mathfrak{R}}\left(0<g_{\mathfrak{R}}<\gamma\right)
$$

where $g_{\mathfrak{R}}$ is the bounded continuous real function in $\Re_{\Re}$ defined by the formula

$$
g_{\mathfrak{R}}=\min _{k=1, \cdots, n}\left[\left(f_{k}^{*}-\boldsymbol{\alpha}_{k}\right)\left(\beta_{k}-f_{k}^{*}\right)\right]
$$

and $\gamma>\left\|g_{\Re}\right\|$. As an $H$-space which is a continuous image of the bicompact $H$-space $\Re$, the space $\Re_{\mathfrak{N}}$ must also be bicompact. In case $\mathfrak{N}=\mathfrak{M}$, the construction of $\Re_{\mathfrak{M}}$ is identical with that described in Theorem 77 , so that $\Re_{\mathfrak{M}}$ coincides with $\Re^{*}$ and is topologically equivalent to $\Re$. If $\mathfrak{R}_{1} \supset \mathfrak{R}_{2}$, the relations $G_{\Re_{1}} \supset G_{\Re_{2}}, \mathfrak{X}_{\Re_{1}}(\mathfrak{r}) \subset \mathfrak{X}_{\Re_{2}}(\mathfrak{r})$ are obviously valid. It follows immediately that the correspondence $\mathfrak{X}_{\Re_{1}}(\mathfrak{r}) \rightarrow \mathfrak{X}_{\Re_{2}}(\mathfrak{r})$ from $\Re_{\Re_{1}}$ to $\Re_{\Re_{2}}$ is univocal and continuous; and that $\Re_{\Re_{2}}$ is a continuous image of $\Re_{\Re_{1}}$. By inspection of the construction of the analytical subring $\mathfrak{N}^{*}$ generated by $\mathfrak{N}$, it is evident that every function in $\mathfrak{R}^{*}$ is constant on each set $\mathfrak{X}_{\mathfrak{R}}(\mathfrak{r})$ and hence that $\mathfrak{X}_{\mathfrak{R}^{*}}(\mathfrak{r}) \supset \mathfrak{X}_{\mathfrak{R}}(\mathfrak{r})$. The relation $\mathfrak{R}^{*} \supset \mathfrak{N}$ implies that $\Re_{\mathfrak{N}}$ is a continuous image of $\Re_{\mathfrak{R}^{*}}$ under the correspondence $\mathfrak{X}_{\mathfrak{N}^{*}}(\mathfrak{r}) \rightarrow \mathfrak{X}_{\mathfrak{N}}(\mathfrak{r})$ where $\mathfrak{X}_{\mathfrak{N}^{*}}(\mathfrak{r}) \subset \mathfrak{X}_{\mathfrak{N}}(\mathfrak{r})$. We see therefore that $\mathfrak{X}_{\mathfrak{N} *}(\mathfrak{r})=\mathfrak{X}_{\mathfrak{N}}(\mathfrak{r})$ and that the indicated correspondence is biunivocal. An elementary proposition now shows that the bicompact $H$-spaces $\Re_{\Re}$ and $\Re_{\Re^{*}}$ are topologically equivalent. $\dagger$

In order to obtain a deeper insight into the subject matter of Theorem 81, we shall next prove a generalization of the Weierstrass approximation theorem.

THEOREM 82. In order that $\Re_{\mathfrak{R}}$ be topologically equivalent to $\Re_{\mathfrak{M}}$ and to $\Re$ by virtue of the correspondences $\mathfrak{r} \rightarrow \mathfrak{X}_{\mathfrak{M}}(\mathfrak{r}) \rightarrow \mathfrak{X}_{\mathfrak{N}}(\mathfrak{r})$, it is necessary and sufficient that $\mathfrak{N}^{*}=\mathfrak{M}$.

$\dagger$ AH, p. 95, Satz III. 
The usual statement of the Weierstrass approximation theorem can be broken down into two propositions: (1) every function continuous in a closed interval of $R$ can be uniformly approximated by a "polynomial" constructed from the function $f(\alpha)=\alpha$ and the real numbers by the algebraic operations and the formation of absolute values; (2) the function $f(\alpha)=|\alpha|$ can be uniformly approximated by a polynomial $p(\alpha)$ where $p(0)=0$. It is obvious that $\mathfrak{R}^{*}$ can be constructed from $\mathfrak{N}$ by taking the uniform limits of "polynomials" constructed from $\mathfrak{N}$ and $R$ by the application of the algebraic operations and the formation of absolute values. Hence, if we restrict ourselves to this characterization of $\mathfrak{R}^{*}$, the equation $\mathfrak{N}^{*}=\mathfrak{M}$ may be regarded as a generalization of part (1) of the Weierstrass approximation theorem. The proof which we shall give below will be a proof of this partial generalization; it will be in particular a proof of (1). In order to have a proof of a complete generalization of the Weierstrass approximation theorem, we must eliminate the use of absolute values from the construction of $\mathfrak{R}^{*}$. We can do this, as we have already seen in the discussion of Theorem 75, if and only if we use part (2) of the Weierstrass approximation theorem. Since our proof of the generalization of part (1) will be an algebraico-topological proof, we may regard (2) as the "analytical kernel" of the Weierstrass approximation theorem for general topological spaces.

From Theorem 81 we already know that $\Re_{\Re}$ and $\Re_{\Re^{*}}$ are topologically equivalent. Hence there is no loss of generality in assuming that $\mathfrak{R}=\mathfrak{N}^{*}$, in other words, that $\mathfrak{N}$ is an analytical subring. From Theorem 81 it is then known that the relation $\mathfrak{N}=\mathfrak{M}$ implies the topological equivalence of $\Re_{\mathfrak{R}}, \Re_{\mathfrak{R}}$, and $\Re$. Hence we have only to prove that the topological equivalence of $\Re_{\mathfrak{R}}$ and $\mathfrak{R}_{\mathfrak{R}}$ implies $\mathfrak{N}=\mathfrak{M}$ when $\mathfrak{N}$ is an analytical subring of $\mathfrak{M}$.

If $f$ is an arbitrary function in $\mathfrak{M}$ and $\epsilon$ is an arbitrary positive number, we shall construct a function $g$ in $\mathfrak{N}$ with the properties $f \leqq g<f+\epsilon$. Since $\mathfrak{N}$ is closed in $\mathfrak{M}$, the resulting inequality $\|f-g\|<\epsilon$ leads to the conclusion that $\mathfrak{N}=\mathfrak{M}$.

As a first step in this construction we show that, if $\alpha$ and $\beta$ are real numbers satisfying the inequality $\alpha<\beta$, then there exists a function $g_{\alpha \beta}$ in $\mathfrak{N}$ which satisfies the inequality $f \leqq g_{\alpha \beta}$ in $\Re$ and the inequality $g_{\alpha \beta} \leqq \beta+\epsilon / 2$ in the closed set $\mathfrak{F}=\Re(\alpha \leqq f \leqq \beta)$. If $\beta+\epsilon / 2 \geqq\|f\|$ or if $\mathfrak{F}$ is void, we may obviously take $g_{\alpha \beta}$ as the constant $\|f\|$; and if $\leftrightarrow=\Re(\alpha-\epsilon / 2<f<\beta+\epsilon / 2)$ coincides with $\Re$, we. may take $g_{\alpha \beta}$ as the constant $\beta+\epsilon / 2$. Hence we may confine our attention to the case where $\beta+\epsilon / 2<\|f\|, \mathfrak{F} \neq 0$, and $\mathscr{G} \neq \Re$. Since $\Re_{\mathfrak{R}}$ is topologically equivalent to $\Re_{\mathfrak{M}}$ and to $\Re$ by hypothesis, and since $\&$ contains $\mathfrak{F}$, each point $\mathfrak{r}$ in $\mathfrak{F}$ determines an open set $\mathfrak{S}_{\mathfrak{N}}(\mathfrak{r})=\Re(\gamma<g<\delta), g \in \mathfrak{N}$, such that $\mathfrak{r} \in \mathfrak{S}_{\mathfrak{N}}(\mathfrak{r}) \subset \mathbb{S}$. Now the function $h=|g-g(\mathfrak{r})| / \eta$, where $0<\eta<\min (g(\mathfrak{r})-\gamma, \delta-g(\mathfrak{r}))$, be- 
longs to $\mathfrak{R}$; and the sets $\mathfrak{S}_{\mathfrak{N}}(\mathfrak{r})=\Re(h<1), \mathfrak{F}_{\mathfrak{N}}(\mathfrak{r})=\Re(h \leqq 1)$ are respectively open and closed in $\Re$ and satisfy the relations $\mathfrak{r} \in \mathfrak{S}_{\mathfrak{R}}(\mathfrak{r}) \subset \mathfrak{F}_{\mathfrak{R}}(\mathfrak{r}) \subset \mathfrak{S}_{\mathfrak{N}}(\mathfrak{r}) \subset \mathbb{G}$. As a closed set in the bicompact $H$-space $\Re$, the subspace $\mathfrak{F}$ is bicompact. Hence there exist points $\mathfrak{r}_{1}, \cdots, \mathfrak{r}_{n}$ in $\mathfrak{F}$ such that

$$
\mathfrak{F} \subset \mathfrak{S}_{\mathfrak{N}}\left(\mathfrak{r}_{1}\right) \mathbf{u} \cdots \cup \mathfrak{S}_{\mathfrak{N}}\left(\mathfrak{r}_{n}\right) \subset \mathfrak{F}_{\mathfrak{N}}\left(\mathfrak{r}_{1}\right) \mathbf{u} \cdots: \mathbf{u} \mathfrak{F}_{\mathfrak{R}}\left(\mathfrak{r}_{n}\right) \subset \mathbb{S} .
$$

If the corresponding functions are $h_{1}, \cdots, h_{n}$ respectively, the function $h=\min \left(h_{1}, \cdots, h_{n}\right)$ belongs to $\mathfrak{R}$ and has the properties

$$
\begin{gathered}
\mathfrak{W}_{\mathfrak{N}}=\mathfrak{R}(h<1)=\mathfrak{W}_{\mathfrak{N}}\left(\mathfrak{r}_{1}\right) \mathbf{u} \cdots \mathbf{u} \mathfrak{S}_{\mathfrak{M}}\left(\mathfrak{r}_{n}\right), \\
\mathfrak{F}_{\mathfrak{M}}=\Re(h \leqq 1)=\mathfrak{F}_{\mathfrak{N}}\left(\mathfrak{r}_{1}\right) \mathbf{u} \cdots \mathbf{u} \mathfrak{F}_{\mathfrak{M}}\left(\mathfrak{r}_{n}\right), \\
\mathfrak{F} \subset \mathfrak{F}_{\mathfrak{N}} \subset \mathfrak{F}_{\mathfrak{N}} \subset \mathfrak{S} .
\end{gathered}
$$

Since $\mathcal{G}^{\prime}$ is a non-void closed subset of the bicompact space $\Re$, the function $h$ has on this set a greatest lower bound $\xi$ attained at some point $\mathfrak{r}$ in $\mathbb{B}^{\prime}$; and the relations $\mathcal{G}^{\prime} \subset \mathfrak{F}_{\mathfrak{R}}^{\prime}=\Re(h>1)$ show that $\xi=f(\mathfrak{r})>1$. We can now define the desired function $g_{\alpha \beta}$ by the formula

$$
g_{\alpha \beta}=\{\|f\|-(\beta+\epsilon / 2)\}\{\min [\xi, \max (h, 1)]-1\} /\{\xi-1\}+(\beta+\epsilon / 2) .
$$

It is evident that $g_{\alpha \beta}$ is in $\mathfrak{N}$. The formula, together with the relations $\beta+\epsilon / 2<\|f\|$ and $\xi>1$, makes it plain that $g_{\alpha \beta}$ satisfies the inequality $\beta+\epsilon / 2 \leqq g_{\alpha \beta} \leqq\|f\|$. Since $\mathcal{B}^{\prime} \subset \Re(h \geqq \xi)$ in accordance with our determination of $\xi$, we see that $g_{\alpha \beta}=\|f\| \geqq f$ in $\mathcal{B S}^{\prime}$; on the other hand, we have $g_{\alpha \beta} \geqq \beta+\epsilon / 2>f$ in $\$$. Hence the inequality $f \leqq g_{\alpha \beta}$ holds at every point in $\Re$. Since $\mathfrak{F} \subset \mathfrak{F}_{\Re}$ $=\Re(h \leqq 1)$ we see that $g_{\alpha \beta}=\beta+\epsilon / 2$ in $\mathfrak{F}$. Thus the function $g_{\alpha \beta}$ has all the properties required.

With the aid of the functions $g_{\alpha \beta}$, we can now construct the desired function $g$. We choose a finite number of open intervals $(\alpha, \beta)$ of positive length $\beta-\alpha$ not greater than $\epsilon / 2$ which cover the range of the given function $f$ in $\mathfrak{M}$. Let the functions $g_{\alpha \beta}$ corresponding to these intervals be denoted by $g_{1}, \cdots, g_{n}$ respectively. The function $g=\min \left(g_{1}, \cdots, g_{n}\right)$ then belongs to $\mathfrak{R}$. It obviously has the property $g \geqq f$ since $g_{k} \geqq f$ for $k=1, \cdots, n$. If $\mathfrak{r}$ is any point in $\Re$, then $f(\mathfrak{r})$ belongs to at least one interval $(\alpha, \beta)$ among those chosen above; and the relations $\alpha<f(\mathfrak{r})<\beta, 0<\beta-\alpha \leqq \epsilon / 2$ show that $\beta<f(\mathfrak{r})+\epsilon / 2$. Hence we have $g(\mathfrak{r}) \leqq g_{\alpha \beta}(\mathfrak{r}) \leqq \beta+\epsilon / 2<f(\mathfrak{r})+\epsilon$; and the inequality $g<f+\epsilon$ holds throughout $\Re$. With this the proof is completed.

We may point out in detail that this proof establishes part (1) of the Weierstrass approximation theorem. If $\Re$ is a closed subinterval of $R$, then the function $f$ given by $f(\rho)=\rho$ has the property that the sets $\Re(\alpha<f<\beta)$ constitute a basis for $\mathfrak{R}$. Hence if we take $\mathfrak{N}$ as the subclass of $\mathfrak{M}$ consisting 
of the function $f$ alone, $\Re_{\mathfrak{R}}$ is topologically equivalent to $\Re$ and $\mathfrak{R}^{*}=\mathfrak{M}$, as we wished to prove.

Before applying the generalized approximation theorem, we shall generalize a theorem established by Banach in the case of separable bicompact $H$-spaces (compact metric spaces). Our proof is necessarily somewhat different from the proof of Banach. $\dagger$

TheOREM 83. If $\Re$ and $\mathfrak{R}^{*}$ are bicompact $H$-spaces and if $\mathfrak{M}$ and $\mathfrak{M}^{*}$ are the corresponding function-rings, then the existence of an isometric correspondence $f \rightarrow U f=f^{*}$ between $\mathfrak{M}$ and $\mathfrak{M}^{*}$ is equivalent to the existence of a topological equivalence $\mathfrak{r} \rightarrow \rho(\mathfrak{r})=\mathrm{r}^{*}$ between $\Re$ and $\Re^{*}$, the two correspondences being connected by the relations

$$
\begin{gathered}
f(\mathfrak{r})=\phi^{*}\left(\mathfrak{r}^{*}\right)\left[f^{*}\left(\mathfrak{r}^{*}\right)-\theta^{*}\left(\mathfrak{r}^{*}\right)\right], \quad U f=f^{*}, \quad \rho(\mathfrak{r})=\mathfrak{r}^{*}, \\
U 0=\theta^{*}, \quad U 1=\phi^{*},
\end{gathered}
$$

where $\left|\phi^{*}\right|=1$. If the relations $U 0=0, U 1=1$ or, equivalently, the relations $\theta^{*}=0, \phi^{*}=1$ are satisfied, then the correspondence $f \rightarrow U f=f^{*}$ is an analytical isomorphism between $\mathfrak{M}$ and $\mathfrak{M}^{*}$.

By an isometric correspondence $U$ is meant one with the property $\|U f-U g\|=\|f-g\|$. It is evident that such a correspondence is biunivocal and has an isometric inverse $U^{-1}$.

If $\rho, \phi^{*}$, and $\theta^{*}$ are given arbitrarily, we define $U$ by the equation $f^{*}=U f=f(\rho(\mathfrak{r})) / \phi^{*}(\rho(\mathfrak{r}))+\theta^{*}(\rho(\mathfrak{r}))$. It is easily verified that $U$ carries $\mathfrak{M}$ isometrically into $\mathfrak{M}^{*}$. With the special choice $\phi^{*}=1, \theta^{*}=0$, it is evident that $U$ determines an analytical isomorphism between $\mathfrak{M}$ and $\mathfrak{M}^{*}$. We may remark that if $U$ defines such an isomorphism the necessary relations $U 0=0, U 1=1$ imply $\theta^{*}=0, \phi^{*}=1$.

When $U$ is given, we define $\theta^{*}=U 0$ and determine a new correspondence $V$ by the relations $f \rightarrow V f=U f-\theta^{*}=f^{*}-\theta^{*}$. It is evident that $V$ carries $\mathfrak{M}$ isometrically into $\mathfrak{M}^{*}$ and that $V$ has the additional property $V 0=0$. A theorem of Mazur and Ulam now shows that $V$ is a linear correspondence, satisfying the relation $V(\alpha f+\beta g)=\alpha V f+\beta V g$.

We now construct the topological equivalence $\rho(\mathfrak{r})$ in terms of the correspondence $V$. If $\mathfrak{r}$ is any point in $\Re$ we denote by $\mathfrak{M}(\mathfrak{r})$ the class of all functions $f$ in $\mathfrak{M}$ which satisfy the equation $|f(\mathfrak{r})|=\|f\|$. It is evident that $\mathfrak{M}(\mathfrak{r}) \supset R$. Also, if $f_{1}, \cdots, f_{n}$ are in $\mathfrak{M}(\mathfrak{r})$, the function $g=\sum_{\nu 1}^{\nu=n} f_{\nu} \operatorname{sgn} f_{\nu}(\mathfrak{r})$ belongs to $\mathfrak{M}(\mathfrak{r})$ and satisfies the relation $\|g\|=\sum_{\substack{\nu=n \\ \nu=1}}\left\|f_{\nu}\right\|$, as we infer from the inequalities

† See Banach, Théorie des Opérations Linéaires, Warsaw, 1932, p. 170, Théorème 3.

¥ A proof is given by Banach, Théorie des Opérations Linéaires, Warsaw, 1932, pp. 166-168. 


$$
\|g\| \leqq \sum_{v=1}^{\nu-n}\left\|f_{\nu}\right\|=\sum_{v=1}^{\nu=n}\left|f_{\nu}(\mathfrak{r})\right|=g(\mathfrak{r}) \leqq\|g\| .
$$

We can now show that the closed sets $\mathfrak{F}^{*}=\Re^{*}\left(\left|f^{*}\left(\mathfrak{r}^{*}\right)\right|=\left\|f^{*}\right\|\right)$, where $f^{*}=V f$ and $f \in \mathfrak{M}(\mathfrak{r})$, have a non-void intersection in $\Re^{*}$. Since $\Re^{*}$ is bicompact, it suffices to prove that the intersection of a finite number of sets $\mathfrak{F}^{*}$ is non-void. If $\mathfrak{F}_{1}^{*}, \cdot, \cdot, \mathfrak{F}_{n}^{*}$ are such sets corresponding to the respective functions $f_{1}, \cdots, f_{n}$ in $\mathfrak{M}(\mathfrak{r})$, we consider the associated function $g$ defined above and its correspondent

$$
g^{*}=V g=\sum_{\nu=1}^{v=n} V\left(f_{\nu} \operatorname{sgn} f_{\nu}(\mathfrak{r})\right)=\sum_{\nu=1}^{\nu=n}\left(V f_{\nu}\right) \operatorname{sgn} f_{\nu}(\mathfrak{r})=\sum_{\nu=1}^{\nu=n} f_{\nu}^{*} \operatorname{sgn} f_{\nu}(\mathfrak{r}) .
$$

Since $g^{*}$ assumes its greatest lower and least upper bounds on the bicompact space $\Re^{*}$, there exists a point $\mathfrak{p}^{*}$ in $\Re^{*}$ such that $\left|g^{*}\left(\mathfrak{p}^{*}\right)\right|=\left\|g^{*}\right\|$. We now observe the relations

$$
\left\|g^{*}\right\|=\left|g^{*}\left(\mathfrak{p}^{*}\right)\right| \leqq \sum_{\nu=1}^{\nu=n}\left|f_{\nu}^{*}\left(\mathfrak{p}^{*}\right)\right| \leqq \sum_{\nu=1}^{\nu=n}\left\|f_{\nu}^{*}\right\|=\sum_{\nu=1}^{\nu=n}\left\|f_{\nu}\right\|=\|g\|=\left\|g^{*}\right\|
$$

and the relations

$$
\sum_{v=1}^{n=n}\left|f_{v}^{*}\left(\mathfrak{p}^{*}\right)\right|=\sum_{v=1}^{v-n}\left\|f_{\nu}^{*}\right\|, \quad\left|f_{k}^{*}\left(\mathfrak{p}^{*}\right)\right| \leqq\left\|f_{k}^{*}\right\|, \quad k=1, \cdots, n .
$$

The latter show that we must have $\left|f_{k}^{*}\left(\mathfrak{p}^{*}\right)\right|=\left\|f_{k}^{*}\right\|$ for $k=1, \cdots, n$. Hence the point $\mathfrak{p}^{*}$ is common to $\mathfrak{F}_{1}^{*}, \cdots, \mathfrak{F}_{n}^{*}$ as we wished to prove. We now let $\mathfrak{r}^{*}$ be a point common to all the sets $\mathfrak{F}^{*}$. It is evident that $f \in \mathfrak{M}(\mathfrak{r})$ and $f^{*}=V f$ imply $f^{*} \in \mathfrak{M}^{*}\left(\mathfrak{r}^{*}\right)$; in other words, that $V$ carries $\mathfrak{M}(\mathfrak{r})$ into a subclass of $\mathfrak{M}^{*}\left(\mathfrak{r}^{*}\right)$. By symmetry, the inverse correspondence $V^{-1}$ carries $\mathfrak{M}^{*}\left(\mathfrak{r}^{*}\right)$ into a subclass of some class $\mathfrak{M}(\mathfrak{p})$. The inclusion relation $\mathfrak{M}(\mathfrak{p}) \supset \mathfrak{M}(\mathfrak{r})$ is now obvious; and it implies that $\mathfrak{p}=\mathfrak{r}$. In fact, if $\mathfrak{p} \neq \mathfrak{r}$, there exists a function $f$ in $\mathfrak{M}$ such that $f(\mathfrak{p})=0, f(\mathfrak{r})=1,0 \leqq f \leqq 1$; and the equation $\|f\|=1$ then shows that $f \in \mathfrak{M}(\mathfrak{r}), f \in \mathfrak{M}(\mathfrak{p})$. We can now infer that $V$ carries $\mathfrak{M}(\mathfrak{r})$ into $\mathfrak{M}^{*}\left(\mathfrak{r}^{*}\right)$; and, further, that $V$ carries the family of all classes $\mathfrak{M}(\mathfrak{r})$ biunivocally into the family of all classes $\mathfrak{M}^{*}\left(\mathfrak{r}^{*}\right)$. This correspondence between the classes $\mathfrak{M}(\mathfrak{r})$ and $\mathfrak{M}^{*}\left(\mathfrak{r}^{*}\right)$ determines a biunivocal correspondence $\mathfrak{r} \longleftrightarrow \mathfrak{r}^{*}=\rho(\mathfrak{r})$ between $\Re$ and $\Re^{*}$. We have to prove that the latter correspondence is a topological equivalence. From the construction of $\rho(\mathfrak{r})$, it is seen that the sets $\Re(|f|=\|f\|)$ and $\Re^{*}\left(\left|f^{*}\right|=\left\|f^{*}\right\|\right)$ correspond under the correspondence $\mathfrak{r} \longleftrightarrow \mathfrak{r}^{*}=\rho(\mathfrak{r})$ when $f$ and $f^{*}$ are connected by the relation $f^{*}=V f$ : for these two sets are specified by the relations $f \in \mathfrak{M}(\mathfrak{r})$ and $f^{*} \in \mathfrak{M}^{*}\left(\mathfrak{r}^{*}\right)$ respectively. Accordingly the complementary sets $\Re(|f|<\|f\|)$ and $\Re^{*}\left(\left|f^{*}\right|<\left\|f^{*}\right\|\right)$ correspond likewise. 
Thus it is sufficient for us to prove that the latter sets constitute bases for the respective spaces $\Re$ and $\Re^{*}$. Since the same discussion applies to both $\Re$ and $\Re^{*}$, we may consider the space $\Re$ alone. Since $\Re$ is a bicompact $H$-space, it is a $C R$-space; and the sets $\Re(\alpha<g<\beta), g \in \mathfrak{M}$, constitute a basis for $\Re$. If $\Re$ has more than one point, we may discard the sets $\Re(\alpha<g<\beta)$ which coincide with $\Re$ or are void, and still have a basis for $\Re$. If $\Re(\alpha<g<\beta)$ is one of the sets retained, we introduce the function

$$
f=1-\max [0,(g-\alpha)(\beta-g)] /\|(g-\alpha)(\beta-g)\|
$$

and show that $\Re(\alpha<g<\beta)=\Re(|f|<\|f\|)$. It is evident that $0 \leqq f \leqq 1$. If $\mathfrak{r}$ is in the complement of $\Re(\alpha<g<\beta)$, we have $(g(\mathfrak{r})-\alpha)(\beta-g(\mathfrak{r})) \leqq 0$ and $f=1$. We infer that $\|f\|=1$. On the other hand, if $\mathfrak{r} \in \Re(\alpha<g<\beta)$, we have $(g(\mathfrak{r})-\alpha)(\beta-g(\mathfrak{r}))>0$ and $f<1$. Thus we have $\Re(\alpha<g<\beta)=\Re(|f|<\|f\|)$, as we wished to prove. It is evident that $f$ is in $\mathfrak{M}$. Thus we have proved that, unless $\Re$ and $\Re^{*}$ are one-element spaces, the correspondence $\mathfrak{r} \longleftrightarrow \mathfrak{r}^{*}=\rho(\mathfrak{r})$ is a topological equivalence; and the exceptional case is trivial.

We now define $\phi^{*}$ as the function $V 1$ in $\mathfrak{M}^{*}$. Since $\left\|\phi^{*}\right\|=\|f\|=1$ and $\Re(|f|=\| f||)=\Re$ for $f=1$, we conclude that $\Re^{*}\left(\left|\phi^{*}\right|=\| \phi^{*}||\right)=\Re^{*}$ and hence that $\left|\phi^{*}\right|=1$. The correspondence $W$ defined by $W f=\phi^{*} V f$ therefore has the properties $|W f|=|V f|,\|W f\|=\|V f\|=\|f\|, W 0=0, W 1=\phi^{*} \phi^{*}=1$, and $W(\alpha f+\beta g)=\alpha W f+\beta W g$. Since the first two of these properties imply $\Re^{*}(|V f|=\| V f||)=\Re^{*}(|W f|=\| W f||)$, we see that the construction of the preceding paragraph leads to the same topological equivalence $\rho$ if we start with $W$ rather than with $V$.

In terms of $W$ the relation between $U, \phi^{*}, \theta^{*}$, and $\rho$ which we wish to establish assumes the equivalent but simpler form $f(\mathfrak{r})=f^{*}\left(\mathfrak{r}^{*}\right)$ where $f^{*}=W f$ and $\mathfrak{r}^{*}=\rho(\mathfrak{r})$. As a first step in proving this relation, we show that $f \geqq 0$ implies $W f \geqq 0$. If $\alpha$ and $\beta$ are the minimum and maximum, respectively, of the function $f$, the relations $0 \leqq \alpha \leqq \beta$ imply that the function $g=\beta-f \geqq 0$ has the number $\beta-\alpha \geqq 0$ as its maximum. Hence $\|g\|=\beta-\alpha$. If we now write $f^{*}=W f=W \beta+W f-W \beta=\beta-W g \geqq \beta-\|W g\|=\beta-\|g\|=\alpha \geqq 0$, we obtain the desired result. As a second step, we prove that $W|f|=|W f|$. Since $|f|-f \geqq 0$, we have $W|f|-W f=W(|f|-f) \geqq 0$ and hence $W|f| \geqq W f$. Similarly $|f|+f \geqq 0$ implies $-W|f| \leqq W f$. We therefore conclude that $W|f| \geqq|W f|$. By symmetry, $W^{-1}|W f| \geqq\left|W^{-1} W f\right|=|f|$. Since $W^{-1}|W f|-|f| \geqq 0$, we have $|W f|-W|f|=W\left(W^{-1}|W f|-|f|\right) \geqq 0$ and hence $|W f| \geqq W|f|$. Combining this inequality with the one obtained above, we conclude that $|W f|=W|f|$. We are now in a position to complete our proof. Let $\alpha$ be the value of $f$ at a fixed point $\mathfrak{r}$ in $\Re$ and let $\beta$ be the maximum of the function $|f-\alpha|$. Then the function $g=\beta-|f-\alpha| \geqq 0$ belongs to $\mathfrak{M}$ and has a maximum $\beta$ at the point $\mathfrak{r}$. 
Hence $\|g\|=\beta$. Since $\mathfrak{r} \in \Re(|g|=\|g\|)$, we see that $\mathfrak{r}^{*}=\rho(\mathfrak{r})$ belongs to the set $\Re\left(\left|g^{*}\right|=\left\|g^{*}\right\|\right)$ where $g^{*}=W g$. Now $g^{*}=W g=W(\beta-|f-\alpha|)=\beta-W|f-\alpha|$ $=\beta-|W f-W \alpha|=\beta-\left|f^{*}-\alpha\right|, \quad\left|g^{*}\right|=|W g|=W|g|=W g=g^{*}$, and $\left\|g^{*}\right\|$ $=\|g\|=\beta$. Hence we see that $\beta-\left|f^{*}\left(\mathrm{r}^{*}\right)-\alpha\right|=g^{*}\left(\mathrm{r}^{*}\right)=\left|g^{*}\left(\mathrm{r}^{*}\right)\right|=\left\|g^{*}\right\|=\beta$, $f^{*}\left(\mathfrak{r}^{*}\right)=\alpha=f(\mathfrak{r})$. This completes the demonstration.

We can now return to the analysis of the results of Theorem 81, obtaining the following additional information.

THEOREM 84. The correspondence $\mathfrak{r} \rightarrow \mathfrak{X}_{\mathfrak{N}}(\mathfrak{r})$ from $\mathfrak{R}$ to $\Re_{\mathfrak{R}}$ described in Theorem 81 induces an analytical isomorphism between $\mathfrak{R}^{*}$ and the functionring $\mathfrak{M}_{\mathfrak{N}}$ for $\mathfrak{\Re}_{\mathfrak{N}}$. No function which belongs to $\mathfrak{M}$ but not to $\mathfrak{\Re}^{*}$ is carried by this correspondence into a single-valued function in $\Re_{\Re}$.

The correspondence $\mathfrak{r} \rightarrow \mathfrak{X}_{\mathfrak{R}}(\mathfrak{r})$ defines a function $f_{\mathfrak{R}}$ in $\Re_{\mathfrak{R}}$ through the relation $f_{\mathfrak{N}}\left(\mathfrak{X}_{\mathfrak{N}}(\mathfrak{r})\right)=f(\mathfrak{r})$. If $f$ belongs to $\mathfrak{N}$ or $\mathfrak{R}^{*}$, as we have already noted, then $f_{\mathfrak{M}}$ is single-valued, bounded and real. If $f$ is in $\mathfrak{N}$, then $f_{\mathfrak{R}}$ is also cont nuous. Thus the induced correspondence $f \rightarrow f_{\mathfrak{R}}$ carries $\mathfrak{R}$ into a subclass $\mathfrak{\Re} \mathbf{i}_{\mathfrak{R}}$ of the function ring $\mathfrak{M}_{\mathfrak{N}}$ for $\mathfrak{\Re}_{\mathfrak{N}}$ in such a way that $|f| \rightarrow\left|f_{\mathfrak{R}}\right|$ and $\|f\|=\left\|f_{\mathfrak{N}}\right\|$. It is obvious that the space $\left(\Re_{\mathfrak{R}}\right)_{\mathfrak{B} \mathfrak{N}}$ is topologically equivalent to $\Re_{\mathfrak{N}}$. Theorem 82 therefore shows that the analytical subring $\left(\mathfrak{P}_{\mathfrak{R}}\right)^{*}$ generated in $\mathfrak{M}_{\mathfrak{N}}$ by $\mathfrak{B}_{\mathfrak{R}}$ coincides with $\mathfrak{M}_{\mathfrak{N}}$. Obviously the correspondence $f \rightarrow f_{\mathfrak{N}}$ can be extended analytically from the classes $\mathfrak{R}, \mathfrak{P}_{\mathfrak{R}}$ to the corresponding classes $\mathfrak{R}^{*},\left(\mathfrak{P}_{\mathfrak{R}}\right)^{*}=\mathfrak{M}_{\mathfrak{R}}$. The extended correspondence is then seen to be an analytical isomorphism between $\mathfrak{N}^{*}$ and $\mathfrak{M}_{\mathfrak{R}}$; it coincides with the correspondence $f \rightarrow f_{\mathfrak{R}}$ defined above for $f \in \mathfrak{R}^{*}$ before we had shown that $f_{\mathfrak{R}} \in \mathfrak{M}_{\mathfrak{N}}$. If $f$ is not in $\mathfrak{R}^{*}$, we wish to show that the corresponding function $f_{\mathfrak{R}}$ defined by $f_{\mathfrak{N}}\left(\mathfrak{X}_{\mathfrak{R}}(\mathfrak{r})\right)=f(\mathfrak{r})$ is not single-valued. To this end we consider the class $\mathfrak{B}$ of all functions in $\mathfrak{M}$ which are constant on each set $\mathfrak{X}_{\mathfrak{R}}(\mathfrak{r}), \mathfrak{r} \in \Re$. Since $\mathfrak{\Re} \supset \mathfrak{R}^{*} \supset \mathfrak{N}$, the space $\Re_{\mathfrak{R}}$ is a continuous image of $\Re_{\mathfrak{B}}$ by virtue of the correspondence $\mathfrak{X}_{\mathfrak{B}}(\mathfrak{r}) \rightarrow \mathfrak{X}_{\mathfrak{R}}(\mathfrak{r})$ as we proved in Theorem 81 . The definition of $\mathfrak{B}$ shows that $\mathfrak{X}_{\mathfrak{B}}(\mathfrak{r})=\mathfrak{X}_{\mathfrak{M}}(\mathfrak{r})$, so that this correspondence is biunivocal. Since $\Re_{\mathfrak{P}}$ and $\Re_{\mathfrak{R}}$ are bicompact $H$-spaces, it follows that they must be topologically equivalent. $\dagger$ If $f \in \mathfrak{P}$, the function $f_{\mathfrak{B}}$ in $\Re_{\mathfrak{B}}$ defined by $f_{\mathfrak{P}}\left(\mathfrak{X}_{\mathfrak{B}}(\mathfrak{r})\right)=f(\mathfrak{r})$ is a bounded continuous real function in accordance with Theorem 81 . The correspondence $\mathfrak{X}_{\mathfrak{B}}(\mathfrak{r}) \rightarrow \mathfrak{X}_{\mathfrak{N}}(\mathfrak{r})$ therefore carries $f_{\mathfrak{B}}$ into the bounded continuous real function $f_{\mathfrak{R}}$ defined by $f_{\mathfrak{R}}\left(\mathfrak{X}_{\mathfrak{M}}(\mathfrak{r})\right.$ ) $=f_{\mathfrak{B}}\left(\mathfrak{X}_{\mathfrak{B}}(\mathfrak{r})\right)=f(\mathfrak{r})$. Since $f_{\mathfrak{N}}$ is in $\mathfrak{M}_{\mathfrak{N}}$, there exists a function $g$ in $\mathfrak{N}^{*}$ such that $g_{\mathfrak{R}}=f_{\mathfrak{R}}$, by virtue of the results established above. Hence we have $f(\mathfrak{r})=f_{\mathfrak{N}}\left(\mathfrak{X}_{\mathfrak{N}}(\mathfrak{r})\right)=g_{\mathfrak{R}}\left(\mathfrak{X}_{\mathfrak{R}}(\mathfrak{r})\right)=g(\mathfrak{r})$ throughout $\Re$; and we conclude that $f \in \mathfrak{N}^{*}$. We have thus shown that $\mathfrak{R}^{*} \supset \mathfrak{B}$. It follows that $\mathfrak{P}=\mathfrak{N}^{*}$. We therefore see

$\dagger$ AH, p. 95, Satz III. 
that, if $f$ is not in $\mathfrak{R}^{*}$, then $f$ is not constant on every set $\mathfrak{X}_{\mathfrak{N}}(\mathfrak{r})$ and that the corresponding function $f_{\mathfrak{R}}$ is not single-valued.

With Theorems 80-84 we have obtained enough information to characterize completely the algebraico-topological structure of the function-rings for bicompact $H$-spaces. We state first the results for the theory of closed ideals.

THEOREM 85. Between the closed ideals in the function-ring $\mathfrak{M}$ for a bicompact $H$-space $\Re$ and the closed subsets of $\Re$, there exists a biunivocal correspondence such that a closed ideal $\mathfrak{R}$ consists of all functions in $\mathfrak{M}$ which vanish on the corresponding closed set $\mathfrak{F}$. The quotient-ring $\mathfrak{M} / \mathfrak{N}$, where $\mathfrak{N}$ is a closed ideal distinct from $\mathfrak{M}$, is isomorphic to the function-ring of the corresponding set $\mathfrak{F}$. Every product of divisorless ideals is a closed ideal; and every closed ideal distinct from $\mathfrak{M}$ is the product of all the divisorless ideals which contain it.

We denote by $(f)$ the set of all points where some function $f$ in a given closed ideal $\mathfrak{N}$ does not vanish; and by $\mathfrak{F}$ the set of all points where every function $f$ in $\mathfrak{N}$ vanishes. It is evident that $\mathfrak{B}$ and $\mathfrak{F}$ are complements of one another. We see also that $\mathbb{S}$ is open: for if $\mathfrak{r} \in(\mathbb{S}$ and $f(\mathfrak{r})=\alpha \neq 0, f \in \Re$, the open set $\Re(\alpha-\epsilon<f<\alpha+\epsilon)$ contains $\mathfrak{r}$ and is contained in $\mathbb{S}$ whenever $0<\epsilon<|\alpha|$. Accordingly, $\mathfrak{F}$ must be a closed set. At this point, it is convenient to show that $\mathfrak{F}=0$ implies $\mathfrak{R}=\mathfrak{M}$. Since $\mathfrak{F}=0$ implies $\mathfrak{H}=\mathfrak{R}$, the open sets $\Re(\alpha<f<\beta)$, where $f \in \Re$ and 0 is outside the closed interval $[\alpha, \beta]$, cover $\Re$. Since $\Re$ is bicompact, we can select a finite number of these sets which also cover $\Re$. If the corresponding functions in $\Re$ are $f_{1}, \cdots, f_{n}$, we see that the function $f=\left|f_{1}\right|+\cdots+\left|f_{n}\right|$ has a positive lower bound in $\Re$. If $f$ were in $\Re$, we could then conclude, since $\mathfrak{N}$ is an ideal, that $1=f \cdot(1 / f) \epsilon \mathfrak{N}$ and hence that $\mathfrak{N}=\mathfrak{M}$. To prove that $f$ actually belongs to $\mathfrak{R}$, it is evidently sufficient to show that $\mathfrak{R}$ contains $|f|$ together with $f$. Since $|f|$ can be uniformly approximated by polynomials of the form

$$
\sum_{\nu=1}^{\nu=n} \alpha_{\nu} f^{\nu}=f\left(\sum_{\nu=0}^{\nu=n-1} \alpha_{\nu+1} f^{\nu}\right)
$$

each of which obviously belongs to the ideal $\mathfrak{N}$ whenever $f$ does, and since $\mathfrak{N}$ is closed, it is evident that $\mathfrak{R}$ has the desired property. Thus $\mathfrak{F}=0$ implies $\mathfrak{N}=\mathfrak{M}$. In the general case, we apply the results of Theorems 81 and 84 by considering the construction and properties of the space $\Re_{\mathfrak{N}}$. The sets $\mathfrak{X}_{\mathfrak{N}}(\mathfrak{r})$ can be characterized as follows: if $\mathfrak{r}$ is in $\mathfrak{S}$, then $\mathfrak{X}_{\mathfrak{R}}(\mathfrak{r})$ consists of the point $\mathfrak{r}$ alone; and, if $\mathfrak{r}$ is in $\mathfrak{F}$, then $\mathfrak{X}_{\mathfrak{N}}(\mathfrak{r})=\mathfrak{F}$. First, if $\mathfrak{r} \in \mathfrak{S}$, there exists a function $f$ in $\mathfrak{N}$ with the property $f(\mathfrak{r}) \neq 0$; and, remembering that $f$ must vanish in $\mathfrak{F}$ and be constant in $\mathfrak{X}_{\mathfrak{N}}(\mathfrak{r})$, we conclude that $\mathfrak{X}_{\mathfrak{R}}(\mathfrak{r})$ is disjoint from $\mathfrak{F}$. Further- 
more if 8 is a point of $\mathbb{S}$ distinct from $\mathfrak{r}$, there exists a function $g$ in $\mathfrak{M}$ with the properties $g(\mathfrak{r})=1, g(\mathbb{g})=0$. The function $h=f g$ belongs to the ideal $\mathfrak{N}$ together with $f$ and has the properties $h(\mathfrak{r}) \neq 0, h(z)=0$. Remembering again that $h$ must be constant in $\mathfrak{X}_{\mathfrak{R}}(\mathfrak{r})$, we conclude that $\mathfrak{X}_{\mathfrak{R}}(\mathfrak{r})$ does not contain 8 . We see therefore that $\mathfrak{X}_{\mathfrak{N}}(\mathfrak{r})=\{\mathfrak{r}\}$. Since the sets $\mathfrak{X}_{\mathfrak{N}}(\mathfrak{r})$ are disjoint, it follows that $\mathfrak{r} \mathfrak{F}$ implies $\mathfrak{X}_{\mathfrak{R}}(\mathfrak{r}) \subset \mathfrak{F}$. On the other hand, the fact that every function in $\mathfrak{N}$ vanishes in $\mathfrak{F}$ implies that $\mathfrak{X}_{\mathfrak{N}}(\mathfrak{r}) \supset \mathfrak{F}$ when $\mathfrak{r} \in \mathfrak{F}$. Consequently, we have $\mathfrak{X}_{\mathfrak{R}}(\mathfrak{r})=\mathfrak{F}$ when $\mathfrak{r} \in \mathfrak{F}$, as we wished to prove. Theorem 84 now shows that the analytical subring $\mathfrak{N}^{*}$ generated by $\mathfrak{N}$ consists of those functions in $\mathfrak{M}$ which are constant on $\mathfrak{F}$ : for such functions are precisely the ones which are constant on each set $\mathfrak{X}_{\mathfrak{R}}(\mathfrak{r})$ and hence remain single-valued on passing from $f$ to $f_{\mathfrak{R}}$ in the manner described in that theorem. On the other hand, we can construct $\mathfrak{N}^{*}$ directly. Let $\mathfrak{B}$ be the class of all functions of the form $f+\alpha$ where $f \in \mathfrak{N}$

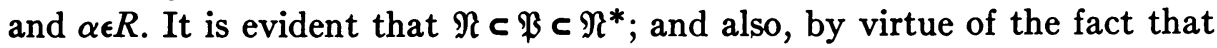
$0 \in \mathfrak{R}$, that $R \subset \mathfrak{B}$. If we can now show that $\mathfrak{B}$ is a closed subring of $\mathfrak{M}$, these relations enable us to conclude that it coincides with $\mathfrak{N}^{*}$ : for the second identifies $\mathfrak{B}$ as an analytical subring; and the first then shows that $\mathfrak{B}=\mathfrak{N}^{*}$. To show that $\mathfrak{B}$ is closed, we recall that we are assuming $\mathfrak{F}$ to be non-void. If $\left\{f_{n}+\alpha_{n}\right\}$ is a convergent sequence in $\mathfrak{B}$, the fact that $f_{n}$ vanishes in $\mathfrak{F}$ shows that the sequences $\left\{\alpha_{n}\right\}$ and $\left\{f_{n}\right\}$ converge separately. Since $\mathfrak{N}$ is a closed subset in the complete metric space $\mathfrak{M},\left\{f_{n}\right\}$ has a limit in $\mathfrak{N}$. Thus $\mathfrak{B}$ is closed. Finally we show that $\mathfrak{B}$ is a subring: if $f+\alpha$ and $g+\beta$ are in $\mathfrak{B}$, then the difference $(f+\alpha)-(g+\beta)=(f-g)+(\alpha-\beta)$ and the product $(f+\alpha)(g+\beta)=(f g+f \beta+g \alpha)+(\alpha \beta)$ are in $\mathfrak{B}$ because the relations $f \in \mathfrak{N}, g \in \mathfrak{N}$, $\alpha \in R, \beta \epsilon R$, and $R \subset \mathfrak{M}$ imply $f-g \epsilon \mathfrak{N}, f g+f \beta+g \alpha \epsilon \mathfrak{N}, \alpha-\beta \epsilon R$ and $\alpha \beta \epsilon R$. Since $\mathfrak{P}=\mathfrak{R}^{*}$, we now infer that every function in $\mathfrak{M}$ which is constant in $\mathfrak{F}$ has the form $f+\alpha$ where $f$ is in $\Re$ and $\alpha$ is its value in $\mathfrak{F}$. In particular, we see that every function $\mathfrak{M}$ which vanishes in $\mathfrak{F}$ belongs to $\mathfrak{R}$. Thus the relation between the ideal $\mathfrak{R}$ and the corresponding closed set $\mathfrak{F}$ is that described in the theorem. The case where $\mathfrak{F}=0, \mathfrak{N}=\mathfrak{M}$ may obviously be included under this statement. On the other hand, we can easily verify that, if $\mathfrak{F}$ is an arbitrary closed set in $\Re$, the class of all functions in $\mathfrak{M}$ which vanish in $\mathfrak{F}$ is a closed ideal $\mathfrak{N}$. Thus the correspondence between closed ideals $\mathfrak{N}$ and closed sets $\mathfrak{F}$ is biunivocal.

In order to determine the nature of the quotient-ring $\mathfrak{M} / \mathfrak{N}$, where $\mathfrak{N}$ is a closed ideal, we consider the associated closed set $\mathfrak{F}$. We may discard the trivial case where $\mathfrak{F}=0, \mathfrak{N}=\mathfrak{M}$. When $\mathfrak{F} \neq 0$, we define the correspondence $f \rightarrow f_{\mathfrak{F}}$, where $f_{\mathfrak{F}}$ is obtained from a given function $f$ in $\mathfrak{M}$ by restricting it to the closed set $\mathfrak{F}$. It is evident that this correspondence determines a homo- 
morphism between $\mathfrak{M}$ and a subring of the function-ring for $\mathfrak{F}$. Since $f_{\mathfrak{F}}$ vanishes in $\mathfrak{F}$ if and only if $f$ is in $\mathfrak{N}$, we see that this subring is an isomorph of $\mathfrak{M} / \mathfrak{R}$. Now if $g$ is any continuous function in $\mathfrak{F}$, a fundamental theorem concerning normal spaces in general and bicompact $H$-spaces in particular shows that there exists a function $f$ in $\mathfrak{M}$ such that $f_{\mathfrak{F}}=g$ in $\mathfrak{F}$. $^{*}$ Hence the indicated subring is identical with the function-ring for $\mathfrak{F}$; and this functionring is an isomorph of $\mathfrak{M} / \mathfrak{R}$. We may remark that $\mathfrak{F}$, being closed in $\mathfrak{R}$, is a bicompact $H$-space.

The intersection or product of divisorless ideals is certainly an ideal; but, since the divisorless ideals in $\mathfrak{M}$ are closed in accordance with Theorem 75 , it is also closed. On the other hand, if $\mathfrak{R}$ is a closed ideal distinct from $\mathfrak{M}$ and $\mathfrak{F}$ is the associated non-void closed set, we see that the divisorless ideals $\mathfrak{A}$ containing $\mathfrak{N}$ are precisely those determined by the points of $\mathfrak{F}$ in accordance with Theorem 80 . Obviously, $f$ belongs to the product of such divisorless ideals if and only if it vanishes in $\mathfrak{F}$; or, in other words, if and only if it belongs to $\mathfrak{R}$. Hence $\mathfrak{R}$ is the product of the divisorless ideals which contain it.

We pass now to the consideration of isomorphism and subrings. Here we merely summarize the results of Theorems $80-84$ in somewhat different language.

THEOREM 86. Two bicompact $H$-spaces are topologically equivalent if and only if their function-rings are analytically isomorphic. One bicompact $H$-space is a continuous image of another if and only if its function-ring is analytically isomorphic to an analytical subring of the function-ring of the other.

Finally we shall state without proof an equivalent of Tychonoff's imbedding theorem for bicompact $H$-spaces. $\dagger$

THEOREM 87. Let $\mathrm{c}$ be an arbitrary infinite cardinal number; let $\mathrm{A}$ be an arbitrary class of cardinal number $c$, for example, the class of all ordinal numbers preceding some suitable (even the first suitable) ordinal number $\omega ;$ let $\Re_{c}$ be the class of all real functions $\mathfrak{r}=\mathfrak{r}(\alpha)$ defined over $\mathrm{A}$, where $0 \leqq \mathfrak{r}(\alpha) \leqq 1$; and let $B_{\mathrm{c}}$ be the class of all sets in $\Re_{\mathrm{c}}$ generated from the special sets $\mathfrak{W}_{\alpha}$, specified by the inequalities $\rho<\mathfrak{r}(\alpha)<\sigma$ where $\rho$ and $\sigma$ are rational numbers, by the formation of finite intersections. By the assignment of each non-void set belonging to $B_{\mathrm{c}}$ as a neighborhood of every one of its points, $\Re_{c}$ becomes a bicompact $H$-space of character c. Every bicompact $H$-space of character not exceeding $c$ is topologically equivalent to a closed subset of $\Re_{c}$; and its function-ring is a homomorph of the functionring of $\Re_{\mathfrak{c}}$, in the sense indicated in Theorem 85.

* AH, pp. 73-76.

$\dagger$ Tychonoff, Mathematische Annalen, vol. 102 (1930), pp. 544-561. 
We point out the rather striking parallel between the results obtained in Theorems $85-87$ for bicompact $H$-spaces and those obtained in Theorems $4,7,9,10$ for Boolean spaces. From this observation, we may surmise that both groups of theorems have the same, essentially algebraic, origin. To discover this common origin, if there be any, it would apparently be necessary to give an abstract characterization of function-rings.

We turn now to the application of the theory of function-rings to problems in the theory of extensions. Some of our results bring out the quite remarkable properties of the bicompact $H$-extension of a $C R$-space which we have already constructed in Theorems 78 and 79: for we can generalize the latter theorem in a quite complete way. We shall now state this generalization.

THeOREM 88. Let $\Re$ be a $C R$-space; let $\mathfrak{Q}$ be the bicompact strict $H$-extension of $\Re$ constructed in Theorems 78 and 79; let $\mathfrak{T}$ be a CR-space which is a continuous.image of $\Re$ by virtue of a correspondence $\mathfrak{t}=\tau(\mathfrak{r})$; and let $\subseteq$ be any bicompact immediate or strict $H$-extension of $\mathfrak{T}$. Then there exists a continuous univocal correspondence $\mathbb{B}=\sigma(\mathfrak{q})$ from $\mathfrak{Q}$ to $\mathfrak{S}$ which coincides in $\Re$ with $\tau(\mathfrak{r})$. In particular, every bicompact immediate or strict $H$-extension of $\Re$ is a continuous image of $\mathfrak{Q}$.

If $f_{\subseteq}$ is any function in the function-ring for. $\subseteq$, the replacement of its argument by $\tau(\mathfrak{r})$ yields a function $f_{\Im}(\tau(\mathfrak{r}))$ in the function-ring for $\Re$. By Theorem 79, the latter function can be extended in a unique way over the space $\mathfrak{Q}$ so as to yield a function $f_{\mathfrak{Q}}$ in the function-ring for $\mathfrak{Q}$. By virtue of the fact that $\Re$ and $\mathfrak{T}$ are everywhere dense in $\mathscr{Q}$ and in $\subseteq$ respectively, the correspondence $f_{\Im} \rightarrow f_{\mathbb{Q}}$ is seen to be an analytical isomorphism between the function-ring for $\subseteq$ and a certain analytical subring of the function-ring for $\mathfrak{Q}$. By Theorem 86 the space $\mathfrak{S}$ is a continuous image of $\mathfrak{Q}$. We examine the relation between $\mathfrak{S}$ and $\mathfrak{Q}$ in greater detail. If $\mathfrak{N}$ is the analytical subring consisting of the functions $f_{\mathfrak{Q}}$, we construct the space $\mathfrak{Q}_{\mathfrak{N}}$ described in Theorem 81. The correspondence $f_{\mathfrak{Q}} \rightarrow f_{\mathfrak{R}}$ defined by $f_{\mathfrak{N}}\left(\mathfrak{X}_{\mathfrak{R}}(\mathfrak{q})\right)=f_{\mathfrak{Q}}(\mathfrak{q}), \mathfrak{q} \in \mathfrak{Q}$, is an analytical isomorphism between $\mathfrak{N}$ and the function-ring for $\mathfrak{Q}_{\mathfrak{N}}$ in accordance with Theorems 81 and 84 . The function-rings for $\mathfrak{S}$ and $\mathfrak{Q}_{\mathfrak{N}}$, being analytically isomorphic to $\mathfrak{R}$, are analytically isomorphic to each other. The correspondence $f_{\mathfrak{R}} \rightarrow f_{\mathfrak{S}}$ therefore defines a topological equivalence $\mathfrak{X}_{\mathfrak{N}}(\mathfrak{q}) \longleftrightarrow \mathbb{B}$ between $\mathfrak{Q}_{\mathfrak{N}}$ and $\mathfrak{S}$ as described in detail in Theorem 83 . Thus the continuous correspondence from $\mathfrak{Q}$ to $\subseteq$ is obtained by eliminating $\mathfrak{X}_{\mathfrak{N}}(\mathfrak{q})$ from the correspondences $\mathfrak{q} \longleftrightarrow \mathfrak{X}_{\mathfrak{N}}(\mathfrak{q}), \mathfrak{X}_{\mathfrak{N}}(\mathfrak{q}) \longleftrightarrow \mathbb{B}$. For a point $\mathfrak{r}$ in $\Re$, we have $f_{\mathfrak{R}}\left(\mathfrak{X}_{\mathfrak{R}}(\mathfrak{r})\right)=f_{\mathfrak{Q}}(\mathfrak{r})=f_{\Im}(\tau(\mathfrak{r}))$. Hence we see that the correspondence from $\mathfrak{Q}$ to $\subseteq$ carries $\mathfrak{r}$ into $\tau(\mathfrak{r})$, as we wished to prove.

If we take $\mathfrak{T}=\Re$, we see that $\mathfrak{S}$ is a continuous image of $\mathfrak{Q}$, as before. 
Hence every bicompact immediate or strict $H$-extension $\mathfrak{S}$ of $\Re$ is a continuous image of $\mathfrak{Q}$.

By a quite similar argument we can now complete the information obtained in Theorem 77.

THEOREM 89. If a CR-space $\mathfrak{T}$ is a continuous image of a $T_{0}$-space $\Re$, then $\mathfrak{T}$ is also a continuous image of the associated CR-space $\Re^{*}$ constructed in Theorem 77 .

If $f$ is any function in the function-ring for $\mathfrak{T}$ and if $\tau$ is the continuous correspondence from $\Re$ to $\mathfrak{I}$, then $f(\tau(\mathfrak{r}))$ is in the function-ring for $\Re$; and the correspondence $\mathfrak{r} \rightarrow \mathfrak{X}(\mathfrak{r})$ from $\Re$ to $\Re^{*}$ carries it into a function $f^{*}$ in the function-ring for $\Re^{*}$. If we place $\mathfrak{X}(\mathfrak{r})$ and $t$ in correspondence whenever $\mathfrak{r} \rightarrow \mathfrak{X}(\mathfrak{r})$ and $\mathfrak{r} \rightarrow \mathfrak{t}=\tau(\mathfrak{r})$, we see therefore that the antecedent of the set $\mathfrak{T}(\alpha<f<\beta)$ is the set $\Re^{*}\left(\alpha<f^{*}<\beta\right)$, in accordance with the relation $f^{*}(\mathfrak{X}(\mathfrak{r}))=f(\tau(\mathfrak{r}))$. If $\mathfrak{t}_{1}$ and $\mathfrak{t}_{2}$ are distinct points in $\mathfrak{I}$, there exists a function $f$ which belongs to the function-ring for $\mathfrak{T}$ and has the properties $f\left(\mathrm{t}_{1}\right)=0$, $f\left(\mathrm{t}_{2}\right)=1$. Since the sets $\mathfrak{T}(-1 / 2<f<1 / 2), \mathfrak{T}(1 / 2<f<3 / 2)$ are disjoint, their antecedents $\Re^{*}\left(-1 / 2<f^{*}<1 / 2\right), \Re^{*}\left(1 / 2<f^{*}<3 / 2\right)$ are likewise disjoint. It follows that the correspondence $\mathfrak{X}(\mathfrak{r}) \rightarrow t$ defined above is univocal. Since the sets $\mathfrak{I}(\alpha<f<\beta)$ constitute a basis for $\mathfrak{I}$, and since their antecedents $\Re^{*}\left(\alpha<f^{*}<\beta\right)$ are open, the correspondence is also continuous. Hence this correspondence represents $\mathfrak{T}$ as a continuous image of $\Re^{*}$ as well as of $\Re$.

We shall now consider in some detail the nature of the bicompact immediate $H$-extensions of a $C R$-space and their connections with the mapping theory. First, let us consider a modification of Theorems 78 and 79.

THEOREM 90. If $\Re$ is a CR-space of infinite character $\mathrm{c}$, then $\Re$ has a bicompact immediate, and hence strict, $H$-extension $\mathfrak{O}$ of the same character $\mathrm{c}$.

In the given space $\Re$, there exists a basis of cardinal number $c$ consisting of sets $\Re(\alpha<f<\beta)$ where $f$ belongs to the function-ring $\mathfrak{M}$ for $\Re$. The associated functions $f$ constitute a subclass $\mathfrak{R}$ of $\mathfrak{M}$ with cardinal number not exceeding c. If $\mathfrak{Q}$ is the bicompact immediate $H$-extension of $\Re$ constructed in Theorems 78 and 79, every function in $\mathfrak{M}$ can be extended from $\Re$ to $\mathfrak{Q}$ in accordance with Theorem 79. By this extension, $\mathfrak{M}$ and $\mathfrak{N}$ are replaced by $\mathfrak{M}_{\mathfrak{Q}}$, the function-ring for $\mathfrak{Q}$, and a subclass $\mathfrak{B}=\mathfrak{N}_{\mathfrak{Q}}$ respectively. The construction of Theorem 81 yields a bicompact $H$-space $\mathfrak{Q}_{\mathfrak{B}}$ which is a continuous image of $\mathfrak{Q}$ by virtue of a correspondence $\mathfrak{q} \rightarrow \mathfrak{X}_{\mathfrak{B}}(\mathfrak{q})$. This correspondence carries $\mathfrak{P}$ into a subclass $\mathfrak{N}_{\mathfrak{B}}$ of the function-ring $\mathfrak{M}_{\mathfrak{B}}$ for $\mathfrak{Q}_{\mathfrak{B}}$. The analytical subrings generated by $\mathfrak{P}$ and $\mathfrak{R}_{\mathfrak{P}}$ respectively are analytically isomorphic under this correspondence; and Theorem 84 shows that the analytical subring 
generated by $\mathfrak{N}_{\mathfrak{B}}$ coincides with $\mathfrak{M}_{\mathfrak{P}}$. If we refer to Theorem 82 , we see that the sets $\mathfrak{Q}_{\mathfrak{B}}\left(\alpha<f_{\mathfrak{B}}<\beta\right), f_{\mathfrak{B}} \in \mathfrak{\Re}_{\mathfrak{P}}$, where $\alpha$ and $\beta$ are unrestricted real numbers, constitute a basis for $\mathfrak{Q}_{\mathfrak{B}}$. Obviously, we may restrict $\alpha$ and $\beta$ to be rational without disturbing the stated property. Since the cardinal number of $\mathfrak{N}_{\mathfrak{B}}$ does not exceed $\mathfrak{c}$, we infer that the character of $\mathfrak{Q}_{\mathfrak{B}}$ does not exceed $c$. It is evident that the correspondence from $\mathfrak{Q}$ to $\mathfrak{Q}_{\mathfrak{B}}$ carries $\Re$, which is an everywhere dense subset of $\mathfrak{Q}$, into a set $\Re_{\mathfrak{B}}$ everywhere dense in $\mathfrak{Q}_{\mathfrak{B}}$. Since the correspondence $\mathfrak{q} \rightarrow \mathfrak{X}_{\mathfrak{R}}(\mathfrak{q})$ carries $f(\mathfrak{q})$ into $f_{\mathfrak{B}}\left(\mathfrak{X}_{\mathfrak{B}}(\mathfrak{q})\right.$ ), we see that $\mathfrak{q}=\mathfrak{r} \in \Re$ implies $f(\mathfrak{r})=f_{\mathfrak{B}}\left(\mathfrak{X}_{\mathfrak{B}}(\mathfrak{r})\right), \mathfrak{X}_{\mathfrak{B}}(\mathfrak{r}) \in \Re_{\mathfrak{B}}$. Hence the sets $\Re(\alpha<f<\beta)$ are carried into the sets $\Re_{\mathfrak{B}}\left(\alpha<f_{\mathfrak{B}}<\beta\right)$ whenever $f \in \mathfrak{R}$ and $f_{\mathfrak{P}} \in \mathfrak{\Re}_{\mathfrak{P}}$. We infer that the correspondence $\mathfrak{r} \rightarrow \mathfrak{X}_{\mathfrak{B}}(\mathfrak{r})$ carries $\Re$ into $\Re_{\mathfrak{B}}$ biunivocally and bicontinuously. Hence $\Re$ and $\Re_{\mathfrak{B}}$ are topologically equivalent. Since the character of $\Re_{\mathfrak{B}}$ is $\mathfrak{c}$, the character of $\mathfrak{Q}_{\mathfrak{B}}$ is not less than $\mathrm{c}$. Thus $\mathfrak{Q}_{\mathfrak{B}}$ is the desired extension.

We now return to the direct study of Boolean maps with particular reference to $C R$-spaces.

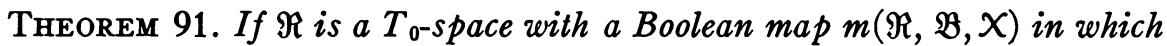
$X$ is a subfamily of a continuous covering family $Z$, then it is a CR-space.

By Theorem 22 , the space $\mathfrak{Q}$ defined by topologizing the family $Z$ is a continuous image of $\mathscr{B}$; and $\mathfrak{Q}$ is therefore a bicompact $H$-space in accordance with Theorem 72 . Since $\mathscr{Q}$ contains $\Re$ as a subspace, it follows that $\Re$ is a $C R$-space.

THEOREM 92. In a $C R$-space $\Re$, let $G$ be a basis consisting of sets $\Re(\alpha<f<\beta)$ where, for each $f$, the corresponding real numbers $\alpha$ and $\beta$ are allowed to range over sets everywhere dense in $R$; and let $A$ be the basic ring for $\Re$ generated by $G$. If $m(\Re, \mathfrak{B}, X)$ is the algebraic map defined by $A$, then there exists a continuous covering family $Z$ in $\mathscr{B}$ which contains $X$ as a subfamily. The topological space $\mathfrak{Q}$ defined by $Z$ is a bicompact immediate $H$-extension of $\Re$. Similarly, if $m(\Re, \mathfrak{B}, X)$ is the irredundant (algebraic) map generated by $A$ through the processes described in Theorems 61-63, the family $X$ can again be imbedded as a subfamily in a continuous covering family $Z$.

We start the proof with a construction like that employed in Theorem 90 , using the class $\mathfrak{R}$ of all functions $f$ occurring in the given basis-sets $\Re(\alpha<f<\beta)$. Thus we imbed $\Re$ as an everywhere dense set in a bicompact $H$-space $\mathfrak{Q}_{\mathfrak{B}}$, in such a way that every function $f$ in $\mathfrak{N}$ can be extended continuously from $\Re$ to $\mathfrak{Q}_{\mathfrak{B}}$ as a function $f_{\mathfrak{B}}$. The sets $\mathfrak{Q}_{\mathfrak{B}}\left(\alpha<f_{\mathfrak{B}}<\beta\right)$ where $f \in \mathfrak{R}$ then constitute a basis for $\mathfrak{Q}_{\mathfrak{B}}$; and this statement remains true even if the numbers $\alpha$ and $\beta$ corresponding to each function $f_{\mathfrak{B}}$ are restricted to everywhere dense sets in $R$. Thus each set $\Re(\alpha<f<\beta)$ determines a corresponding 
set $\mathfrak{Q}_{\mathfrak{B}}\left(\alpha<f_{\mathfrak{B}}<\beta\right)$, the latter sets constituting a basis $G_{\mathfrak{B}}$ in $\mathfrak{Q}_{\mathfrak{P}}$. Since $\Re \Omega_{\mathfrak{B}}\left(\alpha<f_{\mathfrak{B}}<\beta\right)=\Re(\alpha<f<\beta)$, we see that the basic ring $A_{\mathfrak{P}}$ for $\mathfrak{Q}_{\mathfrak{B}}$ generated by the basis $G_{\mathfrak{B}}$ is related to $A$ in the manner described in Theorem 39 for the rings $A$ and $B$ respectively. Hence we can apply Theorem 40 to the study of the maps generated by $A_{\mathfrak{B}}$ and $A$. Since $\Re$ is a $C R$ - and hence an $S R$-space, $\mathfrak{Q}_{\mathfrak{B}}$ is a strict $H$-extension of $\Re$ in accordance with Theorem 65 . Thus we see that the map generated by $A_{\mathfrak{B}}$ can be simplified by the removal of the set $\mathfrak{E}(\mathfrak{b})$ corresponding to the ideal $\mathfrak{b}$ of those members of $A_{\mathfrak{B}}$ which are nowhere dense subsets of $\Re^{\prime}$. The resulting map is equivalent to one obtained from $m(\Re, \mathfrak{B}, \mathcal{X})$ by augmenting the family $\mathcal{X}$. We thus have a map $m\left(\mathfrak{Q}_{\mathfrak{B}}, \mathfrak{B}, Z\right)$. In order to prove the present theorem, it is thus sufficient to show that $Z$ is a continuous covering family. We know that the sets in the family $Z$ are disjoint by virtue of the fact that $\mathfrak{Q}_{\mathfrak{B}}$ is an $H$-space. Using the bicompactness of $\mathfrak{Q}_{\mathfrak{B}}$, we can prove that $Z$ covers $\mathfrak{B}$. Evidently, we can return to the original map $m\left(\mathfrak{Q}_{\mathfrak{B}}, \mathfrak{E}\left(A_{\mathfrak{B}}\right), \Upsilon\right)$ and prove instead that $\Upsilon$ covers $\mathbb{E}\left(A_{\mathfrak{B}}\right)$. If there exists a point $\mathfrak{p}$ in $\mathbb{E}\left(A_{\mathfrak{B}}\right)$ which belongs to no set in $\Upsilon$, we can construct for each set $\mathfrak{Z}$ in $\Upsilon$ a set $\mathbb{E}(a), a \in A_{\mathfrak{P}}$, such that $\mathfrak{V} \subset \mathbb{E}(a), \mathfrak{p} \in \mathbb{E}^{\prime}(a)$. Since the elements $a$ thus obtained constitute a family of subsets of $\mathfrak{Q}_{\mathfrak{B}}$ with the property that their interiors cover $\mathfrak{Q}_{\mathfrak{B}}$, we see that it is possible to select among them certain ones $a_{1}, \cdots, a_{n}$, such that $a_{1} \vee \cdots \vee a_{n}>a_{1}{ }^{\prime \prime} \vee \cdots$ $\vee a_{n}{ }^{\prime \prime}{ }^{\prime}>\mathfrak{Q}_{\mathfrak{P}}$. Since the relation $a_{1} \vee \cdots \vee a_{n}=\mathfrak{Q}_{\mathfrak{B}}$ implies $\mathfrak{p} \in \mathbb{E}\left(A_{\mathfrak{P}}\right)=$ $\&\left(a_{1} \vee \cdots \vee a_{n}\right)=\&\left(a_{1}\right) \cup \cdots \cup \&\left(a_{n}\right)$, we reach a contradiction. Thus $Y$ covers $\mathbb{E}\left(A_{\mathfrak{B}}\right)$, and $\mathcal{Z}$ covers $\mathfrak{B}$. Using the fact that $\mathfrak{Q}_{\mathfrak{B}}$ is necessarily an $R$-space in accordance with Theorem 71, we can now prove that the family $Z$ is continuous. Since $A_{\mathfrak{B}}$ contains the basis $G_{\mathfrak{B}}$ for the $R$-space $\mathfrak{Q}_{\mathfrak{B}}$, Theorem 69 shows that in the map $m\left(\mathfrak{Q}_{\mathfrak{B}}, \mathfrak{F}\left(A_{\mathfrak{B}}\right), \Upsilon\right)$ the family $\Upsilon$ is continuous. Accordingly, $\mathfrak{Q}_{\mathfrak{B}}$ is a continuous image of the bicompact Boolean space $\mathfrak{E}\left(A_{\mathfrak{B}}\right)$ as we see by reference to Theorem 22 . It follows that the removal of the set $\mathfrak{E}(\mathfrak{b})$ from $\mathbb{E}\left(A_{\mathfrak{B}}\right)$ leaves $\mathfrak{Q}_{\mathfrak{B}}$ a continuous image of the set $\mathfrak{E}^{\prime}(\mathfrak{b})$. Hence the map $m\left(\mathfrak{Q}_{\mathfrak{B}}, \mathfrak{B}, Z\right)$ represents $\mathfrak{Q}_{\mathfrak{B}}$ as a continuous image of $\mathfrak{B}$. The family $Z$ is therefore continuous in accordance with Theorem 22.

Since $\mathfrak{Q}_{\mathfrak{B}}$ and $\Re$ are both $C R$-spaces, we can also remove from $\mathfrak{B}=\mathbb{E}(A)$ the set $\mathfrak{E}(\mathfrak{a})$ corresponding to the ideal $\mathfrak{a}$ of all nowhere dense sets in $A$, as described in Theorem 65. We thus obtain maps $m\left(\mathfrak{Q}_{\mathfrak{B}}, \mathbb{F}^{\prime}(\mathfrak{a}), Z\right)$ and $m\left(\Re, \mathcal{E}^{\prime}(\mathfrak{a}), \mathcal{X}\right)$ where $Z$ is an extension of the family $X$. It is evident that $Z$ covers $\mathbb{E}^{\prime}(\mathfrak{a})$; and the argument used above can be applied again to show that $Z$ is continuous.

Theorems 91 and 92 show that the $C R$-spaces are precisely those which have certain Boolean maps with the property that the family $x$ can be ex- 
tended to yield a continuous covering family $Z$. In order to complete the theory, we consider the problem of constructing such an extension in an arbitrary Boolean map.

THEOREM 93. If $m(\Re, \mathfrak{B}, X)$ is an arbitrary Boolean map of a $T_{0}$-space $\Re$ and if $\mathfrak{M}$ is the function-ring for $\mathfrak{B}$, then the class $\mathfrak{R}$ of all functions in $\mathfrak{M}$ which are constant in each set of the family $X$ is an analytical subring of $\mathfrak{M}$. The space $\mathfrak{B}_{\mathfrak{R}}$ constructed by the processes described in Theorem 81 is a continuous image of $\mathfrak{B}$ defined by the Boolean map $m\left(\mathfrak{B}_{\mathfrak{N}}, \mathfrak{B}, X_{\mathfrak{N}}\right)$. Each set $\mathfrak{X}$ in $X$ is contained in a unique set $\mathfrak{X}_{\mathfrak{N}}$ in $\mathfrak{X}_{\mathfrak{N}}$; and the correspondences $\mathfrak{r} \rightarrow \mathfrak{X}(\mathfrak{r}) \rightarrow \mathfrak{X}_{\mathfrak{N}}$, where $\mathfrak{X}(\mathfrak{r}) \subset \mathfrak{X}_{\mathfrak{N}}$, determine a continuous image of $\Re$ in $\mathfrak{B}_{\mathfrak{N}}$. In order that there exist a continuous covering family $Z$ in $B$ which contains the family $X$ as a subfamily, it is necessary and sufficient that $\mathfrak{X} \subset \mathfrak{X}_{\mathfrak{N}}$ imply $\mathfrak{X}=\mathfrak{X}_{\mathfrak{N}}$; when this condition is satisfied, the family $Z$ may be taken as $Z=X_{\Re}$.

The class $\mathfrak{N}$ can be constructed in the following way. If $\mathfrak{X}$ is any set in $X$, it is closed. As in the proof of Theorem 85 , we see that the class $\mathfrak{N}(\mathfrak{X})$ of all functions in $\mathfrak{M}$ which are constant in $\mathfrak{X}$ is an analytical subring. It is obvious that $\mathfrak{N}$ is the intersection of all the analytical subrings $\mathfrak{N}(\mathfrak{X})$. Hence $\mathfrak{N}$ is also an analytical subring; that is, it is a closed subring which contains every constant function. We can now construct $\mathfrak{X}_{\mathfrak{R}}=\mathfrak{X}_{\mathfrak{N}}(\mathfrak{p}), \mathfrak{X}_{\mathfrak{N}}$, and $\mathfrak{B}_{\mathfrak{R}}$ as described in Theorem 81 . It is evident from the construction of $\mathfrak{R}$ that $p \in \mathbb{X}$ implies $\mathfrak{X} \subset \mathfrak{X}_{\mathfrak{N}}(\mathfrak{p})$. In Theorem 81, we saw that the correspondence $\mathfrak{p} \rightarrow \mathfrak{X}_{\mathfrak{N}}(\mathfrak{p})$ from $\mathfrak{B}$ to $\mathfrak{B}_{\mathfrak{N}}$ is univocal and continuous; and Theorem 22 shows that this correspondence defines a Boolean map $m\left(\mathfrak{B}_{\mathfrak{N}}, \mathfrak{B}, \mathfrak{X}_{\mathfrak{R}}\right)$. If we place $\mathfrak{X}_{\mathfrak{N}}$ in correspondence with $\mathfrak{X}$ whenever $\mathfrak{X} \subset \mathfrak{X}_{\mathfrak{N}}$, we obtain a univocal correspondence carrying $\Re$ into a subspace of $\mathfrak{B}_{\mathfrak{R}}$; we identify $\Re$ with the family $X$, topologized in the usual way, of course. This correspondence can also be obtained by restricting the continuous correspondence $\sigma$, from $\mathbb{B}$ to $\mathfrak{B}_{\mathfrak{N}}$, to the union $\subseteq(X)$ of all the sets in $X$. This description of the correspondence shows immediately that it is continuous.

From the results already obtained, it is clear that the family $\chi_{\Re}$ is a continuous covering family. Hence, if $\mathfrak{X} \subset \mathfrak{X}_{\mathfrak{R}}$ implies $\mathfrak{X}=\mathfrak{X}_{\mathfrak{R}}$, we can obtain a continuous covering fämily $Z$ which contains $X$ merely by taking $Z=\mathfrak{X}_{\Re}$. On the other hand, if $X$ has such an extension $Z$, we impose upon $Z$ the usual topology, obtaining a bicompact $H$-space $\mathfrak{Q}$ : for Theorem 22 shows that $\mathfrak{Q}$ is a continuous image of $\mathfrak{B}$; and Theorem 72 then shows that $\mathfrak{Q}$ is a bicompact $H$-space. If $\rho$ is the correspondence from $\mathfrak{B}$ to $\mathfrak{Q}$ and if $f$ is any function in the function-ring for $\mathfrak{Q}$, then $f(\rho(\mathfrak{p}))$ is a bounded continuous function in $\mathbb{B}$ which is constant in each set $\mathbb{Z}$. Since, in particular, $f(\rho(\mathfrak{p}))$ is constant in each set $\mathfrak{X}$, it belongs to the class $\mathfrak{R}$ discussed above. Since $\mathfrak{Q}$ is a bicompact 
$H$-space, it is a $C R$-space. Hence $f$ can be chosen so as to assume distinct values in any two distinct points which we may prescribe. Consequently, the corresponding function $f(\rho(\mathfrak{p}))$ may be chosen so as to assume distinct values in any two distinct sets in $Z$ which we may prescribe. It follows that each set $\mathbb{Z}$ contains a set $\mathfrak{X}_{\mathfrak{N}}$. In particular, we infer that $\mathfrak{X} \subset \mathfrak{X}_{\mathfrak{N}}$ implies $\mathfrak{X}=\mathfrak{X}_{\mathfrak{N}}$, as we wished to show.

\section{HARVARD UNIVERSITY,} Cambridge, Mass. 